UNIVERSIDAD DE BURGOS

DEPARTAMENTO DE INGENIERÍA CIVIL

TESIS DOCTORAL

\title{
EVALUACIÓN DE LA TENACIDAD A FRACTURA EN ACEROS MEDIANTE EL USO DE PROBETAS MINIATURA PREFISURADAS
}

ISIDORO IVÁN CUESTA SEGURA

Burgos, Enero de 2010 
UNIVERSIDAD DE BURGOS

DEPARTAMENTO DE INGENIERÍA CIVIL

TESIS DOCTORAL

\title{
EVALUACIÓN DE LA TENACIDAD A FRACTURA EN ACEROS MEDIANTE EL USO DE PROBETAS MINIATURA PREFISURADAS
}

\author{
Autor: \\ ISIDORO IVÁN CUESTA SEGURA \\ Director: \\ JESÚS MANUEL ALEGRE CALDERÓN
}

Tesis Doctoral presentada en la Universidad de Burgos para la obtención del Título de Doctor

Burgos, Enero de 2010 


\section{AGRADECIMIENTOS}

Desde estas líneas deseo agradecer a todas aquellas personas que de un modo u otro, en mayor o menor medida, han contribuido a la consecución de la presente Tesis.

Especialmente al profesor Jesús Manuel Alegre por su excelente capacidad de dirección, buen humor y cercanía, que ha permitido que este laborioso trabajo se tornase mucho más ameno, y por haber despertado en mí una serie de inquietudes necesarias para su culminación.

Agradecer la financiación recibida del proyecto coordinado MCI Ref: MAT2008-06879C03-03/MAT, así como la confianza que en su día depositaron en nuestro grupo de investigación (GIE) los demás miembros del proyecto, especialmente a las profesoras Covadonga Betegón, Cristina Rodríguez e Inés Peñuelas y al profesor F. Javier Belzunce de la Universidad de Oviedo, y a los profesores Federico Gutiérrez-Solana y José Alberto Álvarez de la Universidad de Cantabria. Haciendo este agradecimiento extensible a los demás miembros de sus respectivos grupos de investigación, principalmente a Emerson Cárdenas (UNIOVI) y Roberto Lacalle (UNICAN).

Al profesor Pedro Bravo, miembro del Grupo de Integridad Estructural de la UBU, por su inestimable ayuda en la realización de los análisis fractográficos pertinentes. También al profesor Luis Sarabia y a la profesora Sagrario Sánchez, del departamento de matemáticas y computación de la UBU, por haberme iniciado en las técnicas de diseño de experimentos, brindándome en todo momento su ayuda desinteresada.

Y como no, a Lorena, a mi madre, a mi padre, a mi hermano y hermanas por mostrarme siempre su apoyo incondicional, tanto en los momentos buenos como en los más difíciles. 


\section{ÍNDICE}

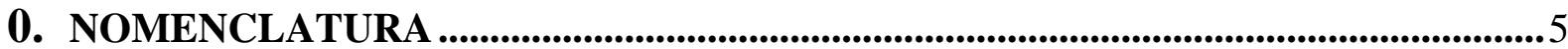

1. INTRODUCCIÓN Y OBJETIVOS .......................................................................................9

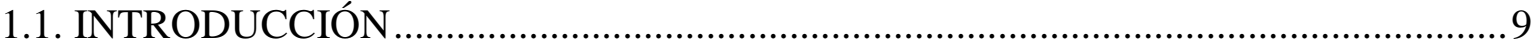

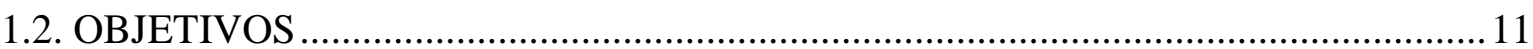

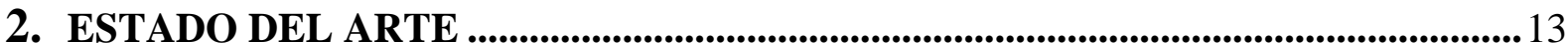

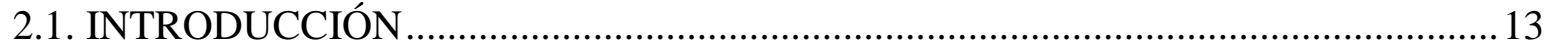

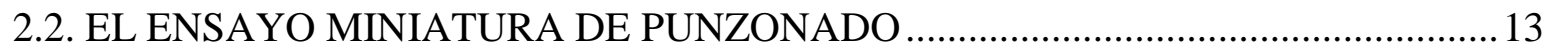

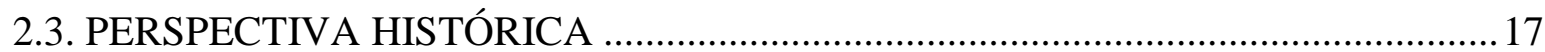

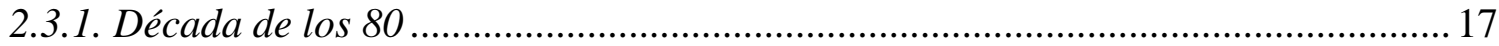

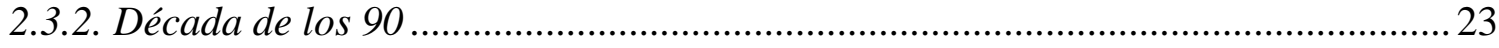

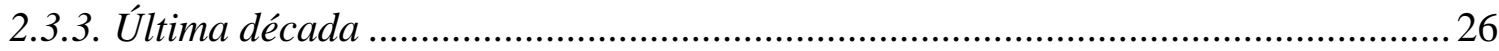

2.4. PROCEDIMIENTOS DE INTEGRIDAD ESTRUCTURAL ........................................33

2.4.1. Aproximación mediante el Diagrama de Fallo, FAD .............................................. 34

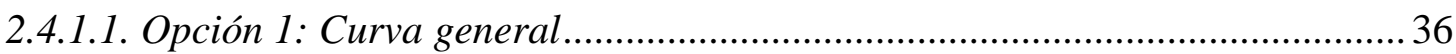

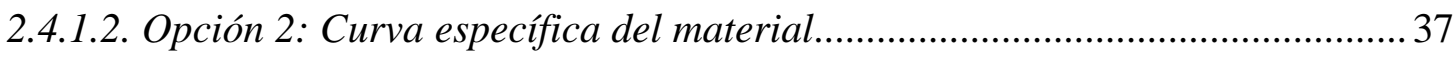

2.4.1.3. Opción 3: Curva obtenida usando un análisis de la integral J.........................38

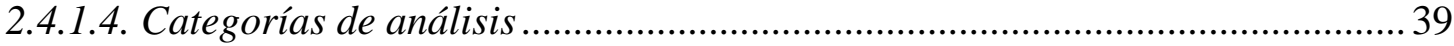

2.4.1.5. Breve descripción de los procedimientos ......................................................... 42

2.4.2. Procedimientos basados en el uso de CDF ........................................................... 45

2.4.3. Procedimientos basados en ambos conceptos.......................................................... 46

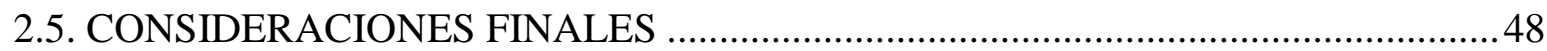

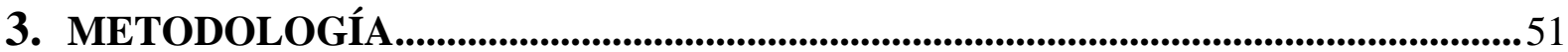

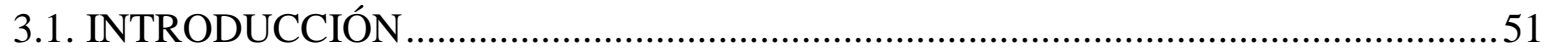




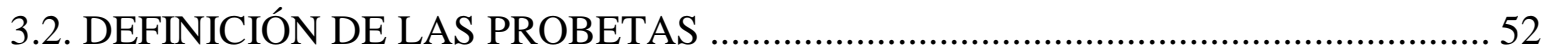

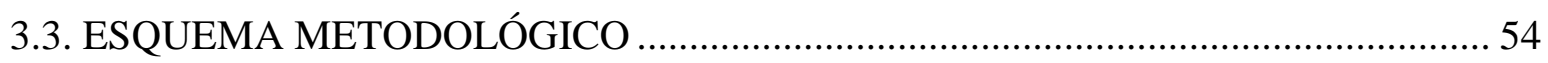

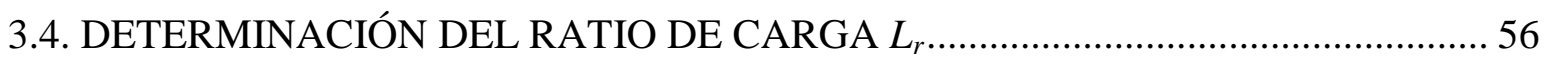

3.4.1. Determinación de la carga aplicada en el inicio de propagación de fisura, $P_{a p l} .56$

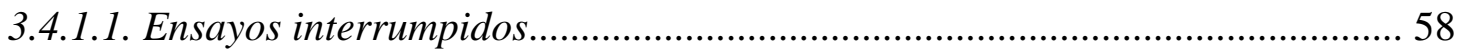

3.4.1.2. Método de evaluación de la pendiente de la curva....................................... 59

3.4.1.3. Método de la flexibilidad o de las descargas elásticas.................................. 61

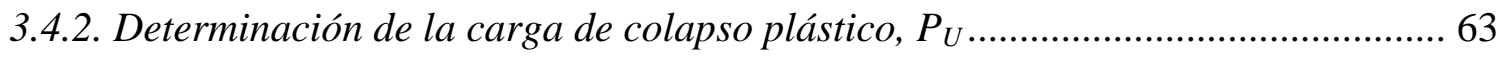

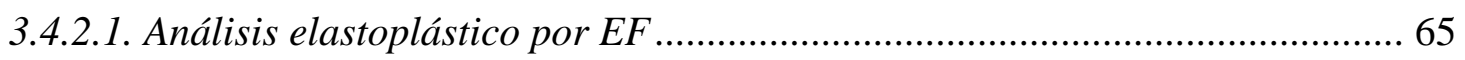

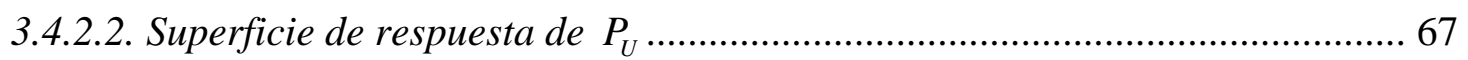

3.5. DETERMINACIÓN DE $K_{r}$ A TRAVÉS DEL DIAGRAMA FAD .............................. 81

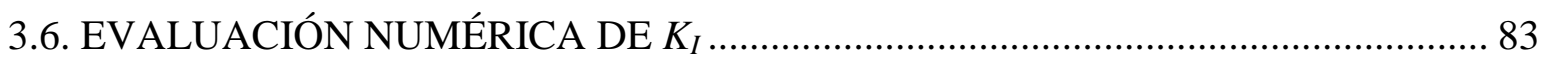

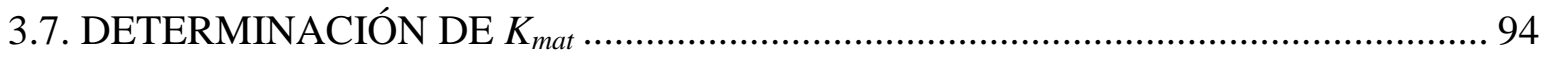

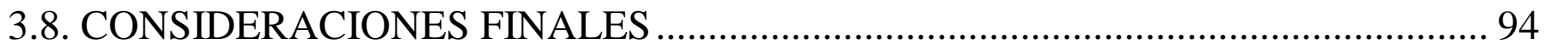

4. CARACTERIZACIÓN DEL MATERIAL ................................................................... 97

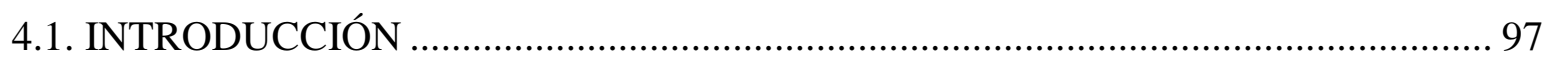

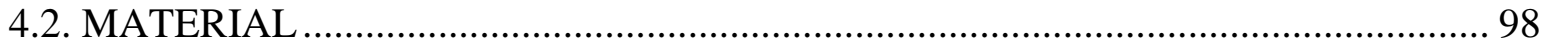

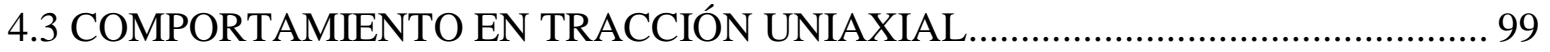

4.4 ESTIMACIÓN DE PROPIEDADES ELASTOPLÁSTICAS ..................................... 104

4.4.1. Estimación de los parámetros $\sigma_{y}, \sigma_{u t s}, n y \sigma_{0.2}$ a partir del SPT....................... 104

4.4.2. Caso práctico de la estimación de los parámetros $\sigma_{y}, \sigma_{u t s}, n$ y $\sigma_{0.2}$ a partir del

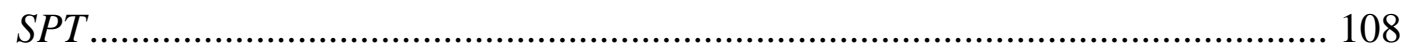

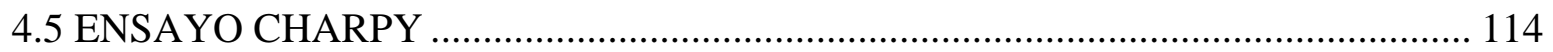

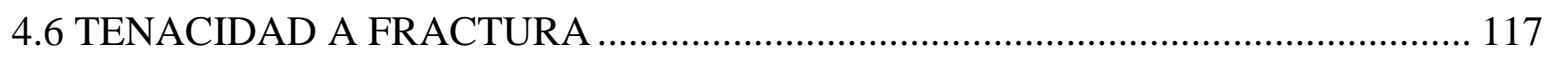

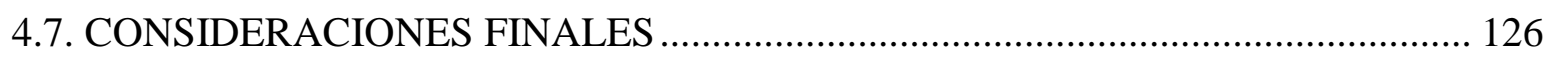

5. APLICACIÓN DE LA METODOLOGÍA Y ANÁLISIS DE RESULTADOS......... 127

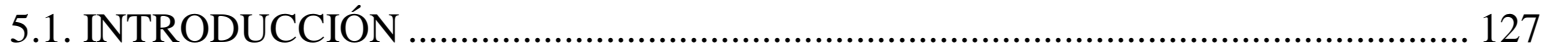

5.2. OBTENCIÓN DE LA CARGA DE INICIO DE PROPAGACIÓN, $P_{a p l} \ldots \ldots . . . . . . . . . . . .129$

5.2.1. Ensayos interrumpidos sobre probetas SPT prefisuradas con relación a/t de 0.54 
5.2.2. Ensayos interrumpidos sobre probetas SPT prefisuradas con relación a/t de 0.4

5.2.3. Método de evaluación de la pendiente de la curva ............................................... 140

5.2.4. Método de la flexibilidad ...................................................................................... 143

5.2.5. Profundidad de fisura de las probetas SPT prefisuradas ................................... 145

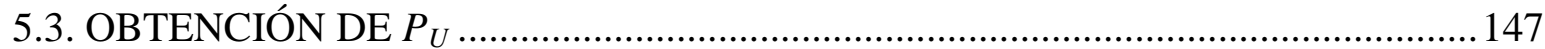

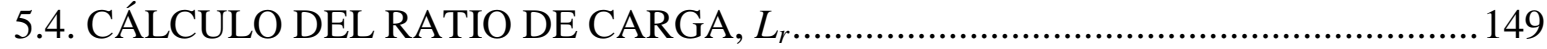

5.5. REPRESENTACIÓN DEL DIAGRAMA DE FALLO, FAD …............................... 150

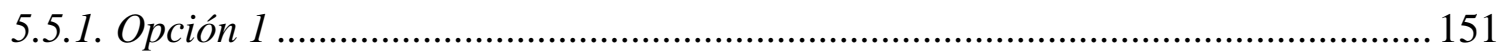

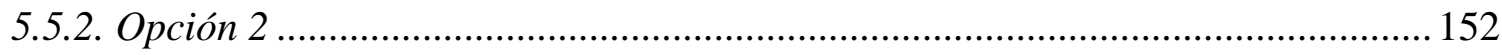

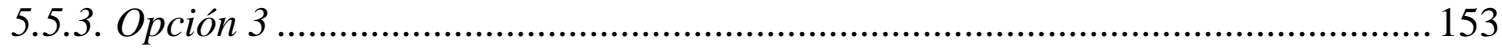

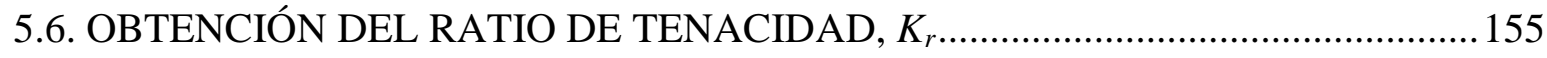

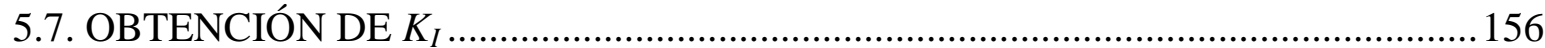

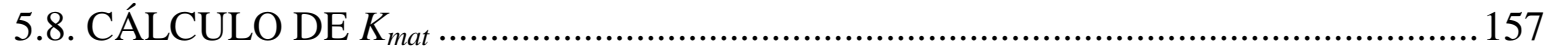

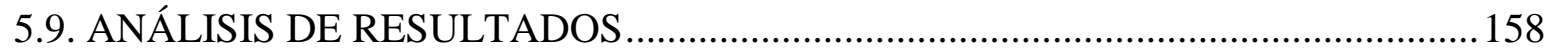

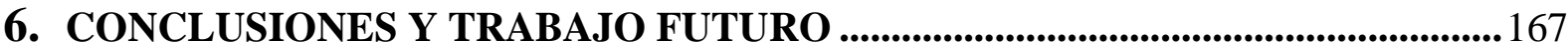

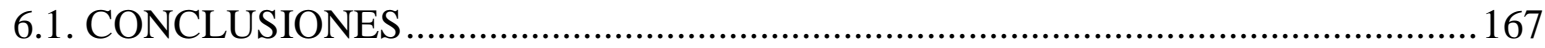

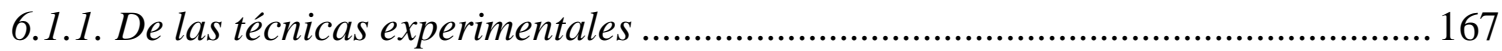

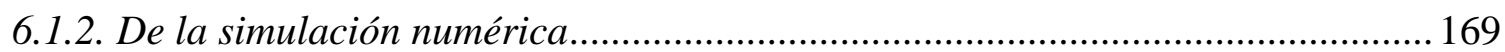

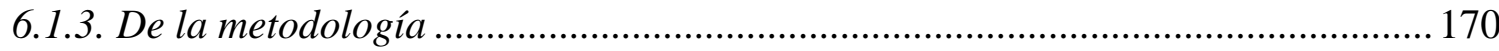

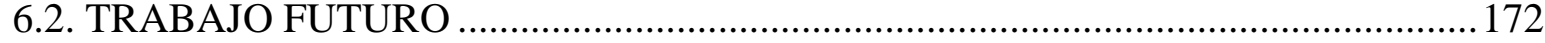

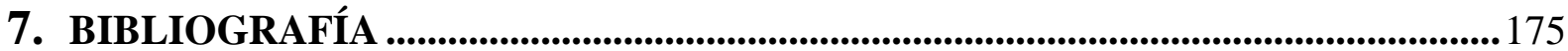





\section{NOMENCLATURA}

$A_{C V}$, Constante de la ecuación tipo tangente hiperbólica

$a[\mathrm{~mm}]$, Profundidad de fisura

$a_{o}[\mathrm{~mm}]$, Fisura inicial en el ensayo de tenacidad a fractura

$a_{p}[\mathrm{~mm}]$, Fisura final en el ensayo de tenacidad a fractura

$a / t^{*}$, Variable codificada de la relación $a / t$

$B[\mathrm{~mm}]$, Espesor de la probeta CT

$B_{C V}$, Constante de la ecuación tipo tangente hiperbólica

$B_{e}[\mathrm{~mm}]$, Espesor efectivo de la probeta CT

$B_{N}[\mathrm{~mm}]$, Espesor neto de la probeta CT

$b[\mathrm{~mm}]$, Lado de la probeta SPT

$b_{o}[\mathrm{~mm}]$, Ligamento remanente inicial en la probeta CT

$b_{0}, b_{1}, b_{2}, b_{3}, b_{11}, b_{22}, b_{33}, b_{12}, b_{13}, b_{23}$, Coeficientes del modelo cuadrático

$C_{C V}$, Constante de la ecuación tipo tangente hiperbólica

$C_{1}$, Constante de la curva $J-R$

$C_{2}$, Exponente de la curva $J-R$

$D_{C V}$, Constante de la ecuación tipo tangente hiperbólica

$d[\mathrm{~mm}]$, Diámetro del punzón

$d_{m}[\mathrm{~mm}]$, Diámetro de la matriz inferior

$E[\mathrm{MPa}]$, Módulo de Young

$E^{\prime}[\mathrm{MPa}]$, Módulo de Young modificado

$E^{*}$, Variable codificada del módulo de Young

$e$, Deformación ingenieril

$f\left(E^{*}, \sigma_{0.2}^{*}, n^{*}\right)$, Modelo cuadrático de superficie de respuesta

$g\left(a / t^{*}, \sigma_{0.2}^{*}, n^{*}\right)$, Modelo cuadrático de superficie de respuesta

$J\left[k J / m^{2}\right]$, Integral $J$ 
$J_{a p p}\left[k J / m^{2}\right]$, Integral $J$ aplicada

$J_{c}\left[\mathrm{~kJ} / \mathrm{m}^{2}\right]$, Tenacidad a fractura sin apenas un crecimiento estable de la fisura

$J_{e}\left[\mathrm{~kJ} / \mathrm{m}^{2}\right]$, Parte elástica de la integral $J$ aplicada

$J_{I c}\left[\mathrm{~kJ} / \mathrm{m}^{2}\right]$, Tenacidad a fractura para condiciones de deformación plana

$J_{\text {limit }}\left[k J / m^{2}\right]$, Integral $J$ límite

$J_{\max }\left[\mathrm{kJ} / \mathrm{m}^{2}\right]$, Integral $J$ máxima

$J_{p}\left[\mathrm{~kJ} / \mathrm{m}^{2}\right]$, Parte plástica de la integral $J$ aplicada

$J_{R}$, Curva resistente del diagrama de fuerza motriz de agrietamiento

$K\left[M P a \cdot m^{1 / 2}\right]$, Tenacidad a fractura

$K_{c}\left[\mathrm{MPa} \cdot \mathrm{m}^{1 / 2}\right]$, Factor de intensidad de tensiones crítico

$K_{g}\left[M P a \cdot m^{1 / 2}\right]$, Tenacidad a fractura, para un pequeño valor de desgarro dúctil

$K_{I}\left[\mathrm{MPa} \cdot \mathrm{m}^{1 / 2}\right]$, Factor de intensidad de tensiones

$K_{I c}\left[M P a \cdot m^{1 / 2}\right]$, Tenacidad a fractura para condiciones de deformación plana

$K_{0.2}\left[M P a \cdot m^{1 / 2}\right]$, Tenacidad a fractura, tras el enromamiento de la punta de fisura de $0.2 \mathrm{~mm}$

$K_{\Omega}\left[M P a \cdot m^{1 / 2}\right]$, Tenacidad a fractura, para un determinado valor de desgarro dúctil

$K_{m a t}\left[M P a \cdot m^{1 / 2}\right]$, Tenacidad a fractura del material

$K_{r}$, Ratio de tenacidad

$K_{1}$, Constante de la ecuación de Ramberg Osgood

$k$, Constante de la ecuación para evaluar el factor de intensidad de tensiones

$k_{1}$, Constante de la ecuación potencial para ajustar el factor de intensidad de tensiones

$k_{2}$, Constante de la curva $J-R$

$L[\mathrm{~mm}]$, Longitud del elemento singular

$L_{r}$, Ratio de carga

$L_{r}^{\max }$, Ratio de carga máximo que define la línea de corte en el diagrama FAD

$M$, Constante en el ensayo de tenacidad a fractura

$\mathrm{m}$, Pendiente de la recta en el método de las dos tangentes

$m_{\text {d.e. }}$, Pendiente de las descargas elásticas 
$n$, Exponente de endurecimiento de la ecuación de Ramberg Osgood

$n^{*}$, Variable codificada de $n$

$\mathrm{n}_{0}$, Ordenada en el origen de la recta en el método de las dos tangentes

$n_{\text {0d.e. }}$, Ordenada en el origen de las descargas elásticas

$n_{1}$, Exponente de la ecuación potencial para ajustar el factor de intensidad de tensiones

$P[k N]$, Carga

$P_{a p l}[k N]$, Carga aplicada en el componente fisurado, en el inicio de fisuración

$P_{\max }[k N]$, Carga máxima de la curva carga-desplazamiento del SPT

$P_{U}[k N]$, Carga de colapso plástico en el componente fisurado

$P_{y}[k N]$, Carga de fluencia de la curva carga-desplazamiento del SPT

$r[\mathrm{~mm}]$, Radio de acuerdo de la matriz inferior

$S_{r}$, Ratio de carga según el procedimiento BS 7910

$S_{r}^{\max }$, Ratio de carga máximo según el procedimiento BS 7910

$s[\mathrm{MPa}]$, Tensión ingenieril

$s_{u}[M P a]$, Tensión ingenieril última, igual que $\sigma_{u t s}$

$t[\mathrm{~mm}]$, Espesor de la probeta SPT

$t_{0}[\mathrm{~mm}]$, Espesor inicial de la probeta SPT

$U[J]$, Energía bajo la curva $P-C O D$

$u_{j}[\mathrm{~mm}]$, Desplazamiento nodal en la dirección $\mathrm{x}$

$v_{i}[\mathrm{~mm}]$, Desplazamiento nodal en la dirección y

$W[\mathrm{~mm}]$, Ancho de la probeta CT

$X_{i}$, Valor real de los parámetros variables

$X_{i N I n f}$, Valor real del nivel más bajo del factor $i$

$X_{i N S u p}$, Valor real del nivel más alto del factor $i$

$\tilde{X}_{i}$, Media de $X_{i N S u p}$ y $X_{i N I n f}$

$x_{i}$, Valor codificado de los parámetros variables

$\alpha$, Parámetro de la ecuación de Ramberg Osgood

$\Delta a[\mathrm{~mm}]$, Incremento de la profundidad de fisura 
$\Delta a_{\max }[\mathrm{mm}]$, Incremento máximo de la profundidad de fisura

$\Delta a_{p}[\mathrm{~mm}]$, Incremento de fisura en la probeta CT

$\Delta a_{Q}[\mathrm{~mm}]$, Incremento de fisura en la probeta CT para el valor $J_{Q}$

$\Delta p[\mathrm{~mm}]$, Desplazamiento del punzón

$\Delta p_{a p l}[\mathrm{~mm}]$, Desplazamiento del punzón en el instante de inicio de propagación de fisura

$\Delta_{M / m}$, Factor de forma entre la probeta SPT de mayor y menor dimensión

$\varepsilon$, Deformación verdadera o real

$\varepsilon_{e}$, Deformación verdadera elástica

$\varepsilon_{p}$, Deformación verdadera plástica

$\varepsilon_{\text {ref }}$, Deformación verdadera para la tensión $L_{r} \cdot \sigma_{0.2}$

$\bar{\varepsilon}_{q f}$, Deformación equivalente de fractura

$v$, Coeficiente de Poisson

$\sigma[M P a]$, Tensión verdadera o real

$\sigma_{r e f}[M P a]$, Tensión de referencia

$\sigma_{u t s}[M P a]$, Tensión ingenieril última, igual que $s_{u}$

$\sigma_{y}[M P a]$, Tensión de fluencia o límite elástico

$\sigma_{0.2}[M P a]$, Límite elástico convencional para una deformación plástica del $0.2 \%$

$\sigma_{0.2}^{*}$, Variable codificada de $\sigma_{0.2}$ 


\section{CAPÍTULO 1}

\section{INTRODUCCIÓN Y OBJETIVOS}

\subsection{INTRODUCCIÓN}

La determinación de las propiedades mecánicas y de fractura de un material, se realiza de forma convencional mediante ensayos normalizados, los cuáles utilizan probetas estándar, como pueden ser las de tracción, Charpy o compactas (CT). En la década de los 80, en el campo nuclear, para solventar el problema que suponía caracterizar material irradiado de componentes en servicio, donde la escasez de material para mecanizar probetas convencionales era un serio inconveniente, se desarrolló el ensayo miniatura de punzonado o Small Punch Test (SPT). Dicho ensayo consiste básicamente en un punzonado sobre una probeta miniatura, mediante un punzón de gran rigidez, estando la periferia de la probeta empotrada por una matriz. Estas probetas miniatura generalmente son cuadradas de dimensiones 10x 10x0.5mm, o bien son circulares de diámetro 3mm. Este reducido tamaño de probeta supone que el ensayo SPT se pueda considerar como un ensayo no destructivo del componente en servicio analizado.

En las últimas décadas han sido numerosos los autores que han centrado sus investigaciones en la utilización del SPT con el fin de obtener las propiedades mecánicas del material, en los casos que no se dispone de una cantidad suficiente de material para poder realizar ensayos normalizados. Su aplicabilidad se ha extendido a otros campos, a parte del nuclear, como por ejemplo a los procesos de soldadura para la evaluación de las propiedades del material en las zonas afectadas térmicamente. 
Actualmente, uno de los grandes retos es la posibilidad de obtener las propiedades a fractura del material con el ensayo de este tipo de probetas miniatura. La gran mayoría de los trabajos sobre este tema hasta el momento han sido realizados con probetas convencionales de SPT, siendo muy pocos los autores que han utilizado probetas prefisuradas. A priori, estas probetas prefisuradas, por tener mecanizada una grieta inicial, podrían suponer una mejor opción a la hora de estimar las propiedades a fractura, ya que tienen una mayor similitud con las probetas convencionales (Charpy o CT) usadas para tal fin.

Por otro lado, actualmente se está extendiendo el uso de los procedimientos de integridad estructural como herramienta para predecir el instante de la rotura en componentes fisurados, haciendo uso de un diagrama de fallo (FAD). En dicho diagrama están inmersos el efecto de la forma y tamaño de fisura, geometría de la pieza y propiedades a fractura del material como su tenacidad a fractura $\left(K_{\text {mat }}\right)$, y teniendo en cuenta además, el efecto del comportamiento plástico del mismo. En este procedimiento se asume que el fallo del componente o probeta se produce en el momento en el que se alcanza la línea de fallo definida por el diagrama FAD, la cuál se puede definir mediante diferentes niveles de análisis, en función de las propiedades del material disponibles.

El presente trabajo pretende establecer un procedimiento integrado entre el ensayo de probetas SPT prefisuradas y el diagrama FAD, con el objetivo de estimar y determinar cuantitativamente la tenacidad a fractura del material. Los pilares fundamentales del procedimiento son la simulación numérica, que como se verá más adelante es clave en la estimación de ciertos parámetros, y la realización de ensayos SPT sobre probetas prefisuradas. La profundización en el cálculo elastoplástico de probetas miniatura prefisuradas, permitirá aportar valores de carga de colapso plástico y de factores de intensidad de tensiones no disponibles hasta el momento en la literatura, mediante la aplicación de las técnicas numéricas de EF y de las técnicas de diseño de experimentos. Dichos valores aportados, facilitarán en gran medida la aplicabilidad del procedimiento, ya que eliminarán la necesidad de llevar a cabo una simulación numérica directa para obtener la carga de colapso plástico y el factor de intensidad de tensiones.

Para validar la metodología presentada, ésta se ha aplicado a un acero inoxidable martensítico endurecido por precipitación utilizado en componentes de altas presiones, evaluando 
posteriormente la similitud que presentan los valores de tenacidad estimados, con los determinados a partir de ensayos convencionales y los propuestos por el código ASME. Una vez validada la metodología presentada, será posible su aplicación a cualquier componente en servicio.

\subsection{OBJETIVOS}

Como se ha podido intuir en el apartado anterior, los principales objetivos de la presente Tesis son los siguientes:

- Desarrollar una metodología que permita la evaluación de la tenacidad a fractura en aceros en aquellos casos en los que no se disponga de material suficiente para llevar a cabo ensayos normalizados.

- Aplicar dicha metodología utilizando un acero perfectamente caracterizado, de tal forma que se analice el grado de precisión obtenido con la implementación de la metodología.

- Estudiar el efecto que la profundidad de fisura inicial tiene en los resultados obtenidos y determinar, si es que lo hay, un tamaño óptimo de profundidad de fisura para la aplicación de la metodología.

- Finalmente, la generación de un procedimiento ingenieril que pueda ser directamente aplicado a otro tipo de aceros, de una manera rápida y sencilla, sin necesidad de llevar a cabo simulaciones numéricas por parte del usuario final.

Para cumplir los objetivos mencionados, la presente Tesis ha sido dividida en seis capítulos, sin tener en cuenta los correspondientes a la nomenclatura y a la bibliografía. En el capítulo actual se han introducido la motivación y los objetivos a alcanzar en este trabajo. El Capítulo 2 recoge las nociones básicas en lo que se refiere al SPT y a los procedimientos de integridad estructural. Ya en el Capítulo 3, se ha desarrollado la metodología propuesta para la evaluación de la tenacidad a fractura del material. El Capítulo 4 incluye la caracterización del material que se ha utilizado para la aplicación de la metodología presentada en el capítulo 
anterior. En el Capítulo 5 se recogen los resultados obtenidos para las probetas SPT de dicho material, así como su correspondiente análisis. El último capítulo contiene las principales conclusiones alcanzadas en el transcurso de la presente Tesis. El esquema de la Figura 1.1 muestra las relaciones existentes entre los diferentes capítulos que conforman la Tesis.

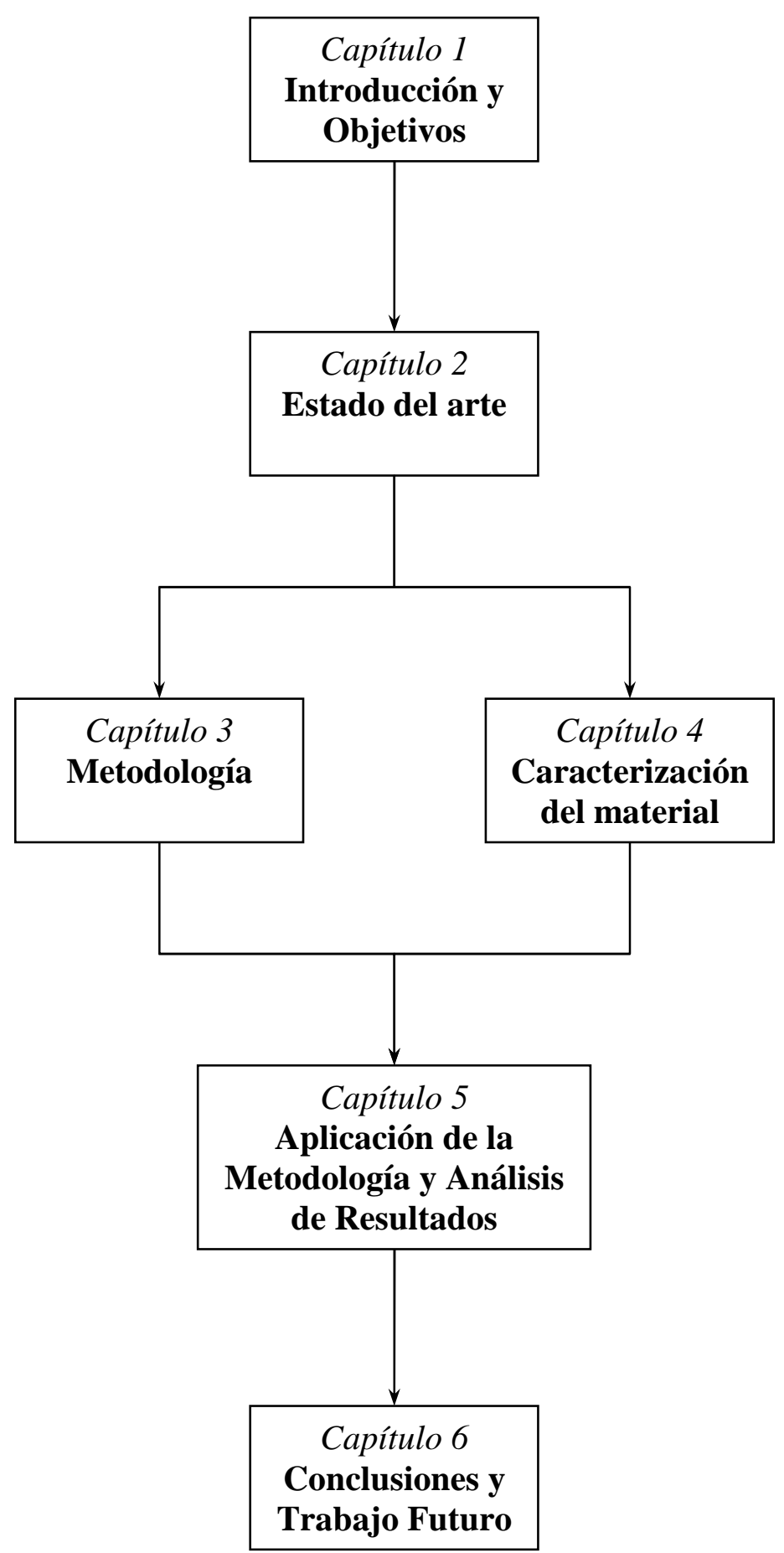

Figura 1.1. Relación existente entre los Capítulos de la presente Tesis. 


\section{CAPÍTULO 2}

\section{ESTADO DEL ARTE}

\subsection{INTRODUCCIÓN}

En el presente capítulo se ha realizado una exhaustiva recopilación de los principales trabajos de investigación que han hecho uso del ensayo miniatura de punzonado, en diversos campos de aplicación, desde sus orígenes en la década de los 80 hasta la actualidad. Por otro lado se han recogido los diferentes procedimientos existentes para la evaluación de la integridad estructural, haciendo especial hincapié en los procedimientos basados en el uso del diagrama FAD, así como en la aplicación de dicho diagrama. Ambos pilares, el ensayo miniatura de punzonado y los procedimientos de integridad estructural, representan la base sobre la que se sustenta la presente Tesis y marcan el camino para alcanzar los objetivos planteados en el capítulo anterior.

\subsection{EL ENSAYO MINIATURA DE PUNZONADO}

El ensayo miniatura de punzonado o Small Punch Test (SPT) comenzó a ser desarrollado en la década de los 80, con el objetivo principal de obtener las propiedades mecánicas del material analizado en aquellos casos en los que no se disponía de material suficiente para la realización de ensayos convencionales. El ensayo consiste básicamente en un punzonado sobre una probeta cuadrada o circular de reducidas dimensiones, mediante un punzón de gran rigidez, estando la periferia de la probeta empotrada por dos matrices. Para evitar 
deformaciones locales muy elevadas en la probeta, la matriz inferior lleva mecanizado un redondeo, cuyo radio de acuerdo es uno de los parámetros con cierta influencia en los resultados obtenidos, como se analizará más adelante. En la actualidad existen diversos utillajes válidos para la realización del ensayo, los cuáles suelen estar diseñados para poder ser acoplados a una máquina universal de ensayo. La compresión de la probeta entre la matriz superior e inferior es de gran importancia para evitar el deslizamiento de la probeta, lo cuál enmascararía los resultados obtenidos, pudiendo dar lugar a una interpretación errónea de dichos resultados. En la Figura 2.1 se presenta un esquema del utillaje de ensayo utilizado en la presente Tesis.

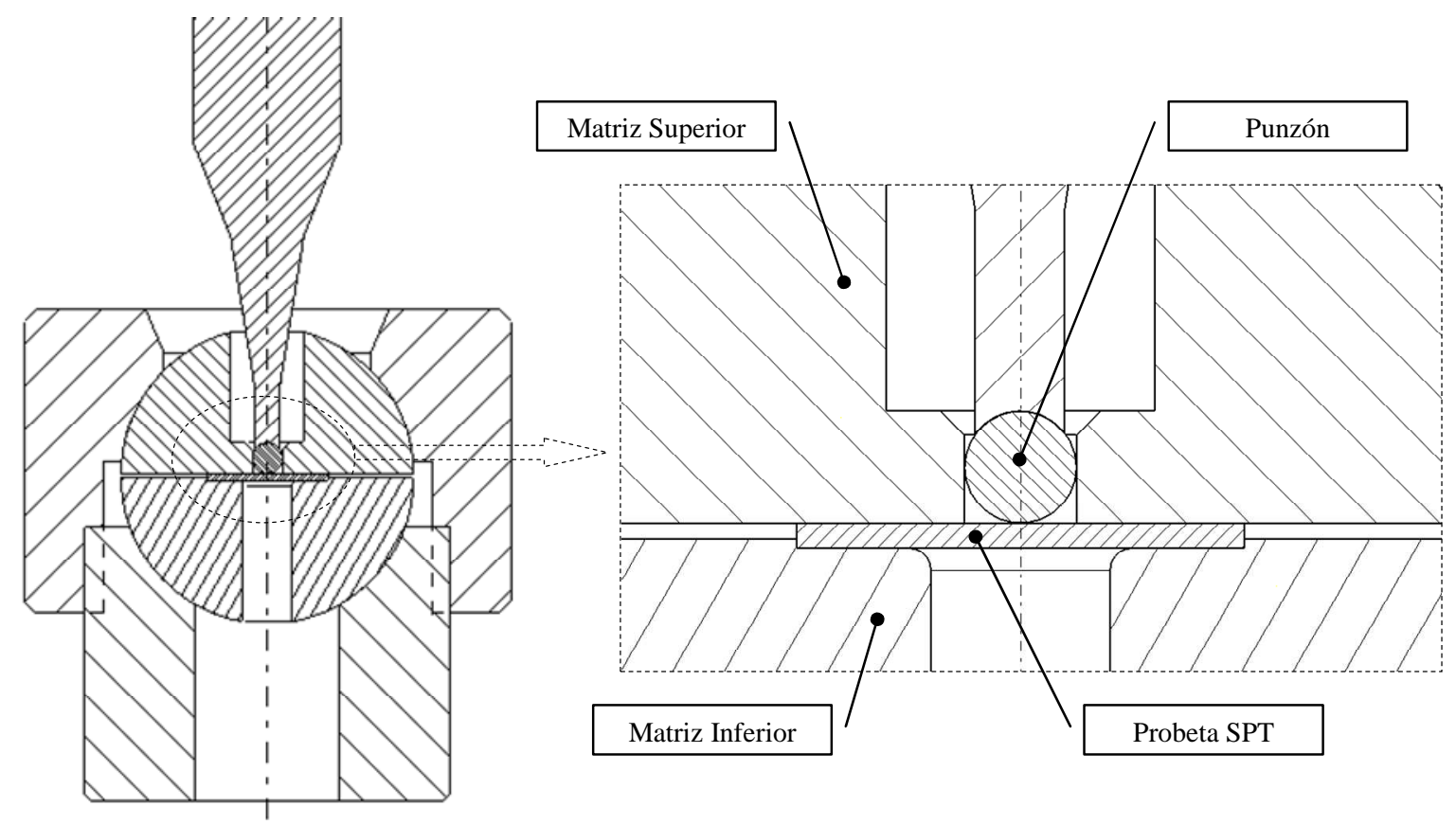

Figura 2.1. Esquema del ensayo SPT.

Durante el ensayo se recogen los valores de carga aplicada y desplazamiento del punzón, tras el correcto tratamiento de los datos almacenados, se obtiene como resultado del ensayo SPT la curva carga-desplazamiento del punzón. Una curva carga-desplazamiento típica para el caso de materiales dúctiles se puede ver en la Figura 2.2, correspondiente a un ensayo de una probeta SPT convencional de 10x10x0.5mm. En general, en dicha curva se pueden diferenciar hasta seis zonas, aunque la finalización de una y el comienzo de la siguiente no se puede determinar exactamente. Cada una de las zonas está influenciada por el comportamiento elastoplástico del material, y se explican en detalle en los siguientes párrafos. 


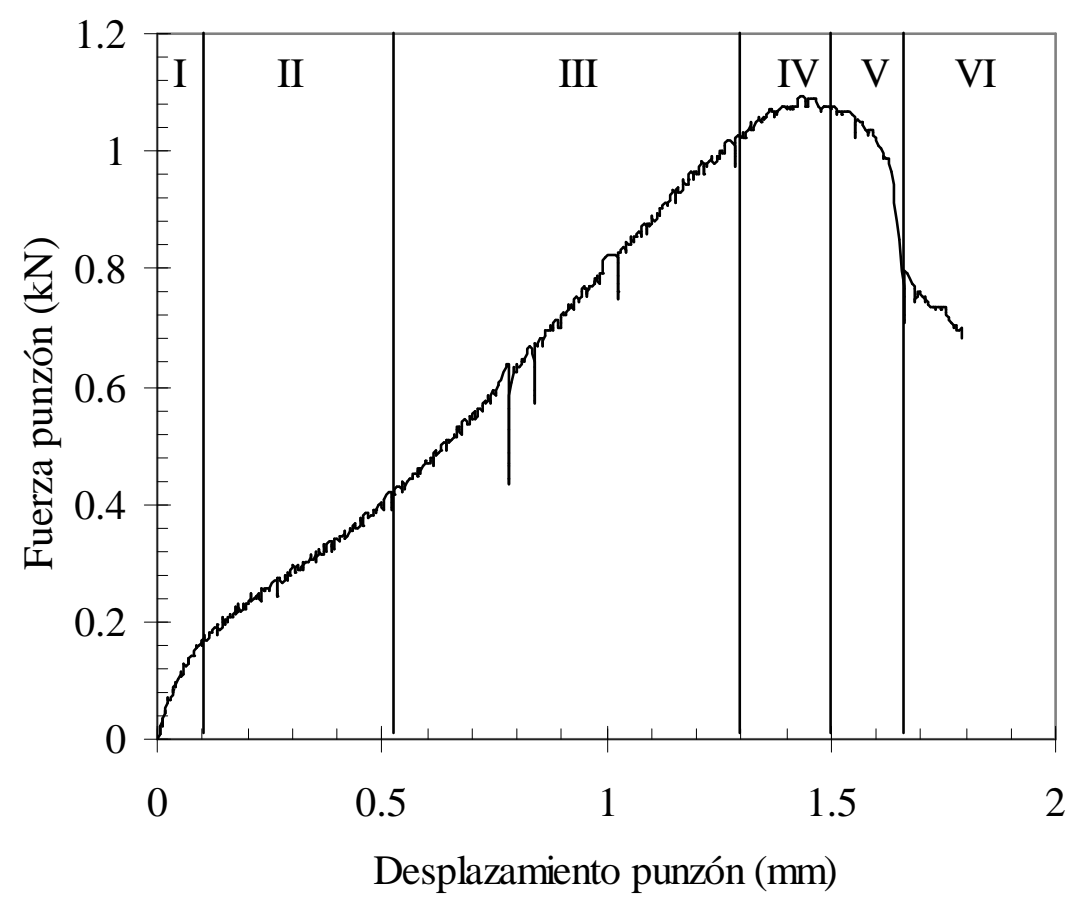

Figura 2.2. Curva carga-desplazamiento típica obtenida en el SPT para el caso de materiales dúctiles.

Zona I. Es la zona en la que el material tiene un comportamiento puramente elástico. Los únicos parámetros del material que tienen influencia en esta zona son los que definen el comportamiento elástico del mismo. Sin embargo, se observa generalmente una ligera influencia debida a la indentación inicial del punzón sobre la probeta.

Zona II. Es la zona de transición elastoplástica. El material comienza a plastificar en algunas zonas de la probeta, fundamentalmente la que se encuentra en contacto con la periferia del punzón, empezando así a tener importancia los parámetros que definen el comportamiento plástico del material. En esta zona también puede comenzar a tener cierta influencia el coeficiente de rozamiento entre la bola y el material ensayado.

Zona III. Es la zona en la que el material alcanza un comportamiento plástico en todos los puntos de la probeta que no están restringidos por las matrices. Podríamos decir que la plastificación va avanzando hasta alcanzar prácticamente toda la probeta. Se alcanzan elevadas deformaciones plásticas en determinadas zonas de la probeta y comienzan a evidenciarse los mecanismos de fractura dúctil. El primero de estos mecanismos que se hace 
presente es la nucleación de huecos en los puntos de mayor deformación plástica, por lo que los parámetros que definen el daño en la probeta empiezan a tener importancia. El daño acumulado en la probeta comienza a crecer conforme va aumentando el desplazamiento del punzón. En muchas ocasiones, el tramo de curva comprendido en esta zona se podría ajustar mediante una recta.

Zona IV. Es la zona en la que se alcanza el valor de carga máxima, en esta zona se produce un adelgazamiento importante del espesor de la probeta. Desde el punto de vista de los micromecanismos dúctiles de fallo se podría decir que comienza la coalescencia de huecos debido a que el daño acumulado alcanza, en algunos puntos de la probeta, el valor crítico. Dicho daño local sigue aumentando hasta que se llega a la carga máxima del punzón, momento en el que en esas zonas comienza la rotura de la probeta, al alcanzar el daño acumulado el valor crítico resistido por el material.

Zona $\boldsymbol{V}$. Es la zona de ablandamiento, una vez que en algunos puntos de la probeta se ha alcanzado la condición de rotura. La rotura de la probeta avanza y la capacidad resistente de la misma disminuye rápidamente. En general, la fisura que se produce en la probeta comienza a crecer de forma circular entorno al centro de la probeta.

Zona VI. Representa la rotura final de la probeta, en la que el punzón la atraviesa por completo. En los ensayos experimentales no es usual continuar hasta la rotura total de la probeta, ya que el ensayo se suele parar cuando se ha alcanzado la zona $\mathrm{V}$ de la curva. Tampoco es a priori una zona de interés, desde el punto de vista de la caracterización del material.

En consecuencia, cada una de las partes en las que se puede dividir la curva cargadesplazamiento parece estar relacionada con una serie de parámetros característicos del material. La primera zona con los parámetros elásticos, la segunda y tercera con las propiedades elastoplásticas y desarrollo del daño local, y la cuarta y quinta con el desarrollo del daño a mayor escala y los parámetros asociados a la rotura del material.

Por último, cabe destacar que en probetas SPT prefisuradas, las primeras regiones en las que se puede dividir la curva carga-desplazamiento coinciden con las comentadas, mientras que las últimas zonas presentan ligeras diferencias, las cuáles se verán más adelante. 


\subsection{PERSPECTIVA HISTÓRICA}

A lo largo de las tres últimas décadas el ensayo SPT ha sido utilizado por numerosos investigadores como base fundamental de sus trabajos. El presente apartado se centra especialmente en recopilar aquellas investigaciones que son verdaderamente afines a los objetivos planteados en la presente Tesis, es decir, los trabajos que han utilizado de uno u otro modo el ensayo SPT para la determinación de las propiedades a fractura del material estudiado. Como se verá mas adelante, hay que esperar hasta el año 2003 para poder comenzar a hablar de la utilización de probetas SPT prefisuradas en la determinación de dichas propiedades del material.

\subsubsection{Década de los 80}

Baik et al. [1] fueron los primeros investigadores en utilizar el SPT con el objetivo de estudiar los efectos que la radiación tenía sobre la temperatura de transición dúctil-frágil en materiales metálicos. Hasta ese momento el ensayo Charpy $(\mathrm{CVN})$ era el método convencionalmente utilizado para la determinación de esa propiedad en materiales metálicos, pero para materiales irradiados las probetas de ese ensayo no era conveniente utilizarlas debido principalmente a su excesivo tamaño para ser irradiadas.

Estos autores realizaron un estudio sobre aceros con diferente microestructura e impurezas dopadas llegando a la conclusión de que existía una única relación lineal entre la temperatura de transición del SPT $\left(T_{S P T}\right)$ y la del CVN $\left(T_{C V N}\right)$ para cada tipo de impureza dopada. La Figura 2.3 muestra los resultados obtenidos en el estudio. Esta relación era independiente de las variables de la microestructura, como pueden ser el tamaño de grano y la dureza, y viene dada por la ecuación (2.1).

$$
T_{C V N}=\alpha_{1} \cdot T_{S P T}+\beta_{1}
$$

Donde $\alpha_{1}$ es un factor de correlación mecánico que está directamente relacionado con el grado de deformación y el estado tensional, y $\beta_{1}$ es el desplazamiento de la temperatura de transición, siendo en este caso en particular $\alpha_{1} \simeq 2.5, \beta_{1} \simeq 400^{\circ} \mathrm{C}$ para el acero dopado con $\mathrm{P}$ y $\beta_{1} \simeq 485^{\circ} C$ para el acero dopado con $\mathrm{Sn}$. Este primer resultado parece indicar que no existe una equivalencia directa entre ambos ensayos. En particular, los valores de tenacidad 
deducidos a partir de ambos ensayos no serán comparables entre sí, al ser diferentes sus temperaturas de transición. Sin embargo, si parece apuntarse la posibilidad de establecer ciertas relaciones a partir de la ecuación (2.1). Evidentemente las diferencias observadas son lógicas dado que se trata de ensayos de velocidad muy diferente y distintas geometrías de probeta, implicando esta última una triaxialidad diferente en cada probeta.
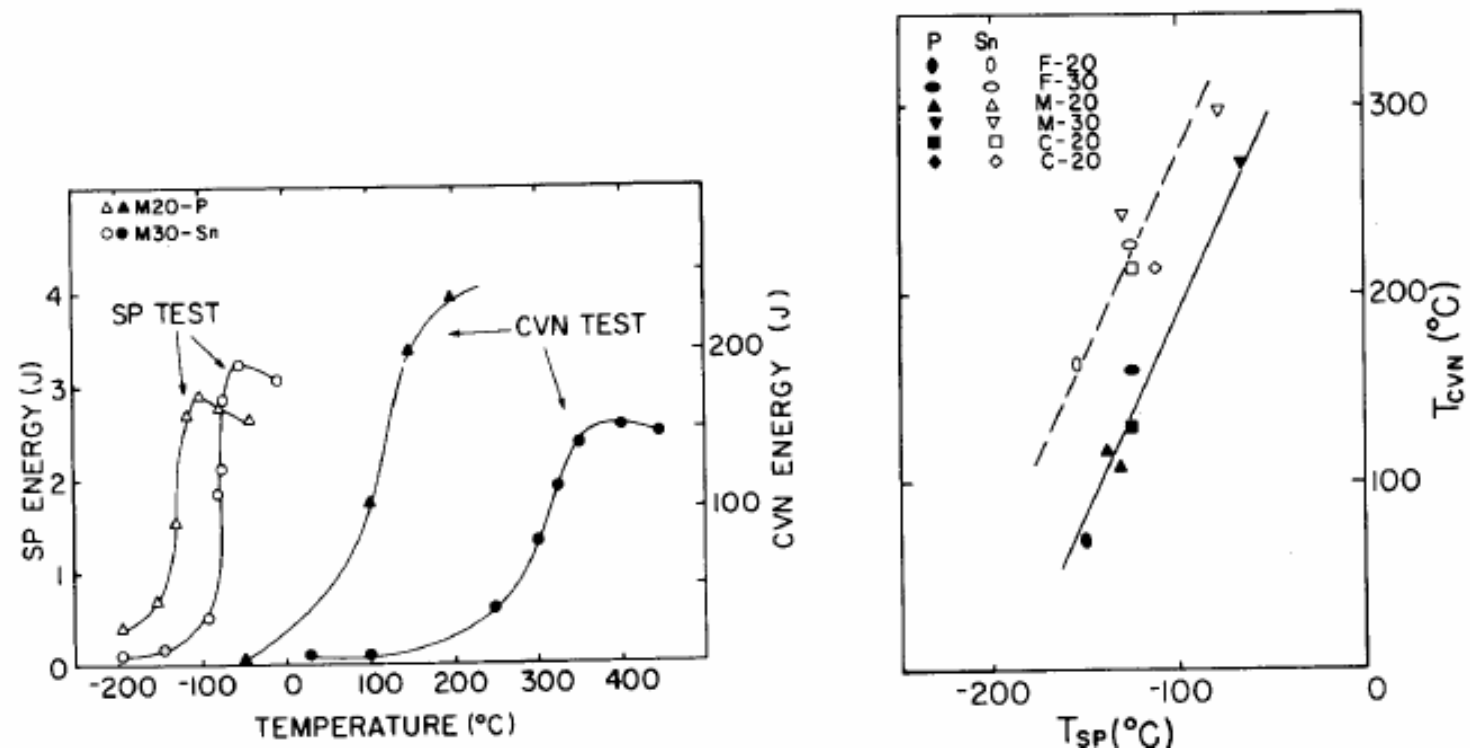

Figura 2.3. Comparación del comportamiento de energía de transición de fractura entre el ensayo CVN y el SPT en aceros que tienen diferentes impurezas dopadas (izquierda), y correlación DBTT entre CVN y SPT en aceros dopados con P y Sn [1] (derecha).

Unos años más tarde en 1986, Kameda et al. [2] ampliaron el estudio anterior [1] centrándose en los aceros ferríticos, los cuáles, eran en esa época ampliamente utilizados en la industria nuclear. La conclusión principal de estos investigadores vino a confirmar la existencia de la correlación lineal entre la DBTT del SPT y la del ensayo CVN para cada uno de los materiales irradiados e impurezas dopadas.

En ese mismo año, Lucas et al. [3] publicaron un trabajo con el objetivo de determinar el efecto de la geometría de la probeta y del utillaje del ensayo en los resultados de los ensayos SPT. Además de utilizar cuatro materiales diferentes, dos tipos de cobre y dos de acero inoxidable austenítico, los parámetros que variaron fueron el espesor de la probeta SPT circular de $3 \mathrm{~mm}$ de diámetro (de $0.1 \mathrm{a} 0.25 \mathrm{~mm}$ ), el diámetro del punzón (de 1 a $1.6 \mathrm{~mm}$ ), el diámetro de la matriz inferior (de 1.3 a $1.9 \mathrm{~mm}$ ) y el radio de acuerdo $($ de 0.2 a $0.5 \mathrm{~mm}$ ) de la matriz inferior. Las principales conclusiones que obtuvieron fueron que incrementando el 
espesor de la probeta se incrementaba la carga de fluencia, la carga máxima y el desplazamiento del punzón en el momento del fallo, y que la localización del fallo pasaba de estar en el centro de la probeta a ser circunferencial. El incremento del diámetro del punzón también hacía aumentar la carga de fluencia y la carga máxima pero hacía decrecer ligeramente el desplazamiento del punzón en el momento del fallo.

Un año más tarde, en 1987, Mao et al. [4,5] comenzaron un trabajo de investigación con el propósito de desarrollar una técnica para extraer información de las propiedades mecánicas y de fractura de materiales a partir del SPT con probetas circulares TEM de $\varnothing 3 \mathrm{~mm}$ x0.25 $\mathrm{mm}$ y probetas cuadradas de 10x10x0.5 mm. Para ello estudiaron el proceso de deformación, introduciendo un nuevo parámetro en el ensayo SPT denominado deformación equivalente de fractura $\left(\bar{\varepsilon}_{q f}\right)$, el cuál fueron capaces de relacionarlo con la tenacidad a fractura $\left(J_{I c}\right)$. Los materiales usados en los ensayos fueron los aceros aleados denominados SUS316, PCA y HT60, y usaron otros aceros y aleaciones para la validación de sus conclusiones, como el A533B y el HT-9.

Utilizaron dos métodos para calcular la $\overline{\mathcal{E}}_{q f}$, el primero midiendo el cambio de espesor en la zona más fina de la probeta una vez realizado el ensayo, según la ecuación (2.2) y el segundo utilizando la relación empírica definida por la ecuación (2.3).

$\overline{\mathcal{\varepsilon}}_{q f}=\ln \left(t_{0} / t\right)$

$\bar{\varepsilon}_{q f}=\beta\left(\delta^{*} / t_{0}\right)^{2}$

Donde $t$ y $t_{0}$ son los espesores de la probeta en la sección más fina en el instante final e inicial respectivamente, $\beta$ es una constante determinada experimentalmente y $\delta^{*}$ es la deflexión de la probeta en el momento de la fractura. La condición necesaria para aplicar cualquiera de estos dos métodos era que la rotura fuese con forma circunferencial. Una vez calculada la $\overline{\mathcal{E}}_{q f}$ observaron la existencia de una relación lineal entre este parámetro y la tenacidad $J_{I c}$, que aparece reflejada en la Figura 2.4, convirtiéndose de esta manera la $\overline{\mathcal{E}}_{q f}$ en un parámetro de fractura muy interesante a la hora de obtener $J_{I c}$ a través del ensayo SPT. 
También, a partir de los ensayos SPT realizados determinaron la tensión de fluencia $\sigma_{y}$ y la tensión última $\sigma_{u t s}$ del material, en función de la carga de fluencia $\left(P_{y}\right)$ y de la carga máxima $\left(P_{\max }\right)$ de la curva carga-desplazamiento respectivamente, estableciendo las ecuaciones (2.4) y (2.5), pioneras en la determinación de $\sigma_{y}$ y $\sigma_{u t s}$ a partir del ensayo SPT. Tal y como se puede ver en la Figura 2.5 con dichas expresiones fueron capaces de ajustar adecuadamente los resultados obtenidos para los diferentes materiales utilizados, las cuáles siguen utilizándose en la actualidad brindando una posible opción a la hora de estimar $\sigma_{y}$ y $\sigma_{u t s}$ a través del ensayo SPT, en aquellos casos en los que no sea posible llevar a cabo ensayos convencionales para determinar dichos parámetros. Se puede observar como la correlación para la estimación del límite elástico es muy buena, pero para el caso de la tensión última la dispersión obtenida es mayor.

$\sigma_{y}=360 \cdot P_{y} / t_{0}^{2}$

$\sigma_{u t s}=130 \cdot P_{\max } / t_{0}^{2}-320$

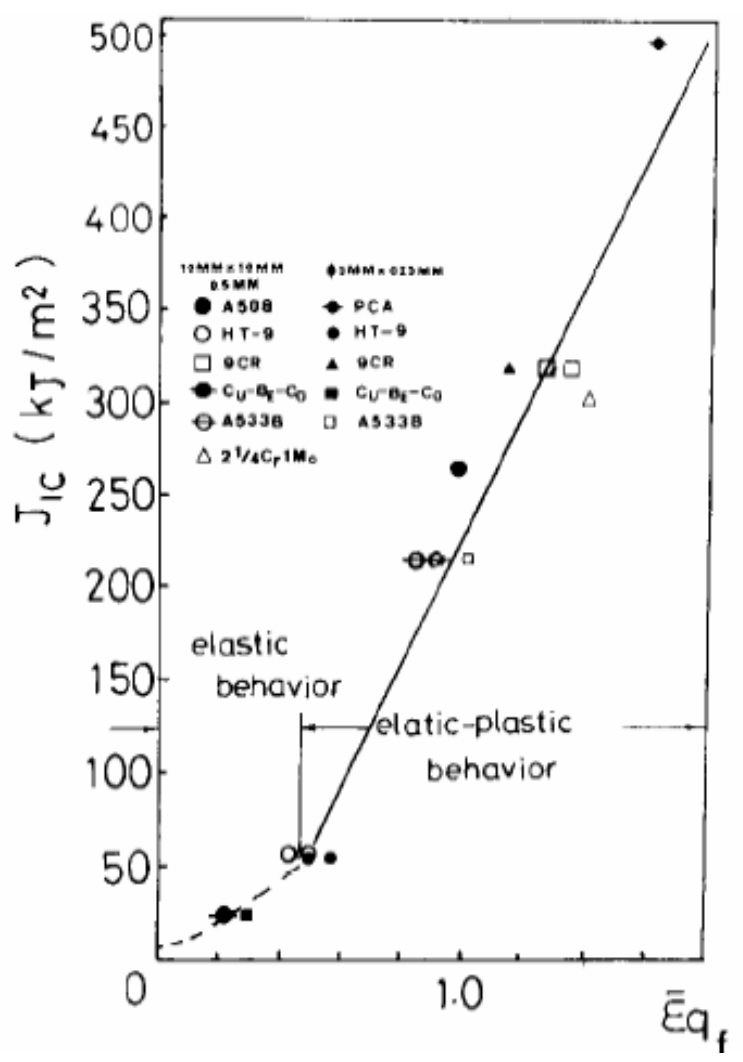

Figura 2.4. Relación entre $\overline{\mathcal{E}}_{q f}$ y $J_{\text {Ic }}$ determinada por Mao y Takahashi [4]. 

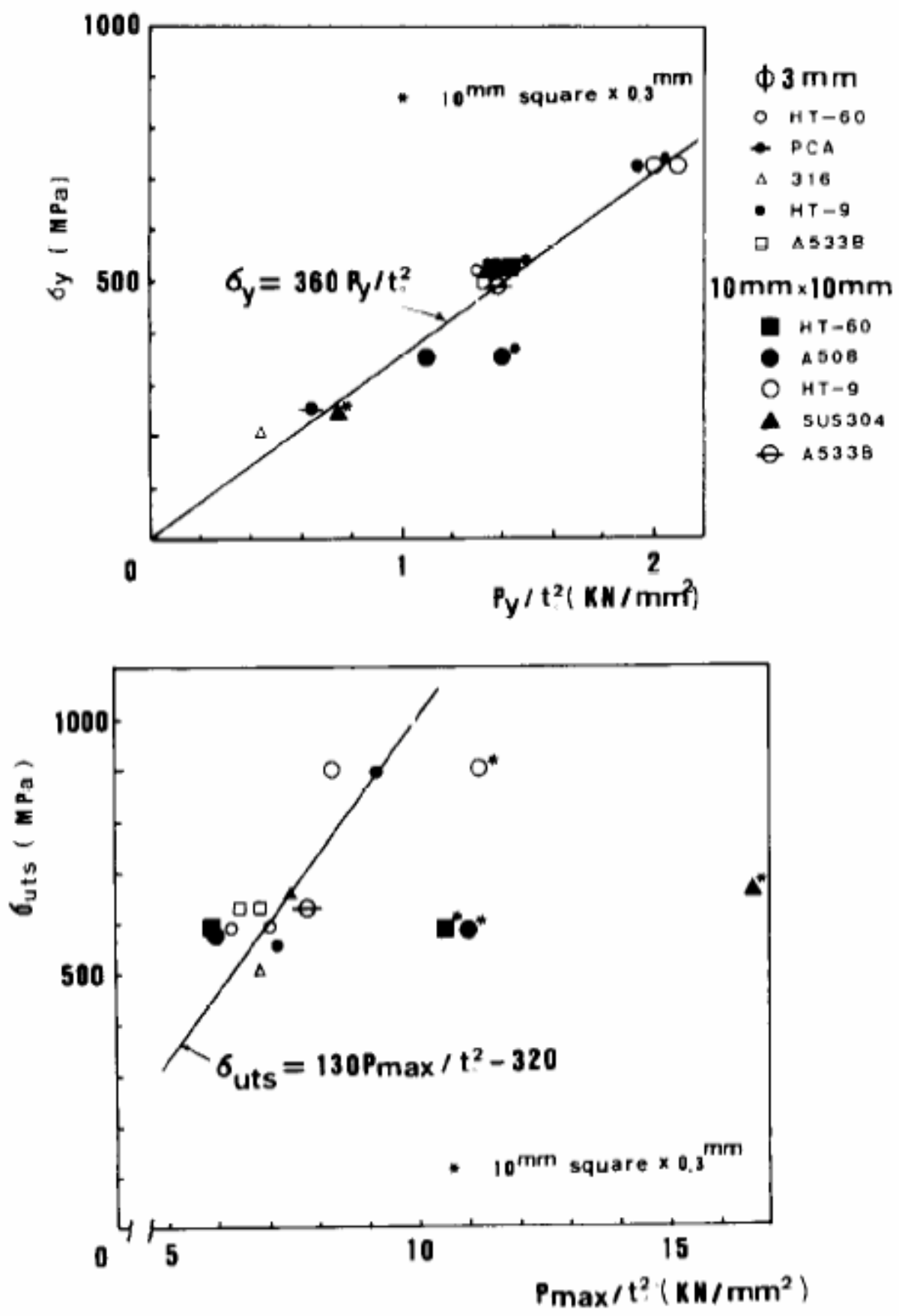

Figura 2.5. Relaciones empíricas obtenidas por Mao y Takahashi [4].

En los últimos años de la década de los 80 y principios de los 90, Misawa et al. [6-10] desarrollaron un sistema de evaluación de degradación por irradiación en aleaciones estructurales candidatas a ser el material de reactores de fusión. Dicho sistema consistía en la utilización del SPT para la determinación de la DBTT, la tenacidad a fractura, la evaluación de la tensión de fallo por corrosión (SCC) y la fragilización por hidrógeno. Los ensayos se 
realizaron sobre aceros ferríticos como el HT-9 y el JFMS y sobre aceros austeníticos como el Tipo 304. En particular, el estudio de la tenacidad a fractura lo realizaron sobre aceros austeníticos a 4.2, 77 y $299 K$. A partir de las expresiones (2.2) y (2.3) determinadas por Mao et al. $[4,5]$ desarrollaron la ecuación (2.6) para la determinación de $\bar{\varepsilon}_{q f}$, donde $\beta=0.088 \mathrm{y}$ $x=1.95$ se obtienen empíricamente a partir de la regresión lineal entre $\ln \cdot\left[\ln \left(t_{0} / t\right)\right] \mathrm{y}$ $\ln \left(\delta / t_{0}\right)$, la cual se puede observar en la Figura 2.6.

$$
\overline{\mathcal{E}}_{q f}=\ln \left(t_{0} / t\right)=\beta\left(\delta^{*} / t_{0}\right)^{x}
$$

Una vez determinado el valor de $\bar{\varepsilon}_{q f}$, el valor de $J_{I c}$ se obtiene, al igual que en los trabajos anteriores, a partir de una regresión lineal independiente de la temperatura, donde el coeficiente de regresión $\mathrm{k}$ toma un valor de $845 \mathrm{~kJ} / \mathrm{m}^{2}$. La Figura 2.6 muestra esta regresión, donde las líneas discontinuas delimitan un intervalo de confianza del 95\%.
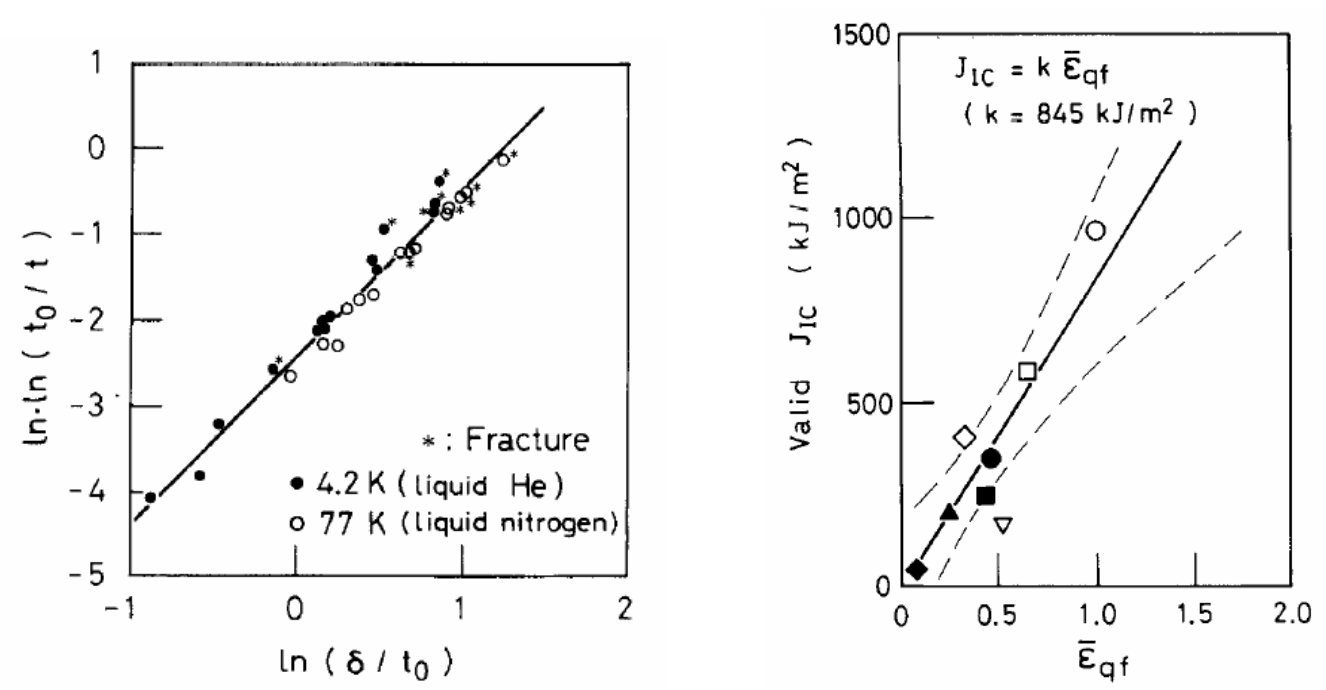

Figura 2.6. Relación entre $\ln \cdot \ln \left(t_{0} / t\right)$ y $\ln \left(\delta / t_{0}\right)$ (izquierda); y regresión lineal independiente de la temperatura entre $J_{I c}$ y $\overline{\mathcal{E}}_{q f}$ (derecha), para aceros austeníticos [8].

Estos estudios representan los primeros intentos de obtener el valor de la tenacidad a fractura del material a partir del ensayo SPT, sirviendo como base de la mayoría de las investigaciones que se llevaron a cabo en los años posteriores. En 1990, Lucas [11] en una recapitulación de los avances que se habían logrado hasta el momento en los ensayos miniatura sobre materiales irradiados, dedica un apartado al SPT en el que hace especial hincapié en dichos estudios. 


\subsubsection{Década de los 90}

En los primeros años de la década de los 90, Mao et al. [12-14], siguiendo con sus investigaciones, realizaron una serie de estudios con el objetivo de obtener un procedimiento de ensayo SPT para estimar $K_{I c}$ en materiales cerámicos, como por ejemplo el SiC, PSZ, $\mathrm{Al}_{2} \mathrm{O}_{3}, \mathrm{Si}_{3} \mathrm{~N}_{4}$, etc. y $J_{\text {Ic }}$ en aceros, como por ejemplo el HT-9, A533B, A508, todos ellos utilizados en la industria nuclear. Los resultados de su trabajo se pueden observar en la Figura 2.7, la cual muestra la relación lineal de $K_{I c}$ y $J_{I c}$ con la tensión de fractura del SPT $\left(\sigma_{f(S P)}\right)$ y $\overline{\mathcal{E}}_{q f}$ respectivamente. Cabe destacar que el nuevo parámetro introducido por estos autores para materiales frágiles, la tensión de fractura del SPT $\left(\sigma_{f(S P)}\right)$, la determinaron a partir de la expresión (2.5) que define el valor de $\sigma_{u t s}$.

En 1991 Suzuki et al. [15], utilizando como base los trabajos anteriores publicados por algunos de ellos [6-10], realizaron un estudio sobre el acero $2 \frac{1}{4} \mathrm{Cr}$-1Mo irradiado por neutrones para la evaluación de la degradación de la tenacidad a fractura. Los resultados que obtuvieron fueron similares a los obtenidos previamente [6-10].

Posteriormente Kameda et al. realizaron una serie de investigaciones enfocadas a determinar las propiedades mecánicas en materiales irradiados [16] y en aleaciones con recubrimientos cerámicos [17]. Los resultados obtenidos ponían de manifiesto que el SPT era útil para evaluar el endurecimiento, la DBTT y el decrecimiento de $J_{I c}$ causado por la irradiación de neutrones. En cuanto a los recubrimientos cerámicos observaron principalmente que un tratamiento de recubrimiento con Ni-P podía llegar a incrementar un $40 \% \sigma_{y}$ y disminuir ligeramente $J_{I c}$ en comparación con el material sin recubrir.

Chi et al. [18] basándose en un trabajo anterior de Mao et al. [14] realizaron ensayos SPT sobre probetas de acero $12 \mathrm{Cr}-1 \mathrm{MoV}$ irradiadas con protones $(16 \mathrm{MeV})$. La Figura 2.8 muestra los resultados obtenidos relacionando $J_{I c}$ con $\overline{\mathcal{E}}_{q f}$, donde también se puede observar la dependencia de la temperatura con la energía absorbida en el SPT con el material irradiado y sin irradiar. Las líneas discontinuas de la gráfica de la izquierda corresponden al intervalo de confianza del 95\% de la relación obtenida por Mao et al. [14]. 

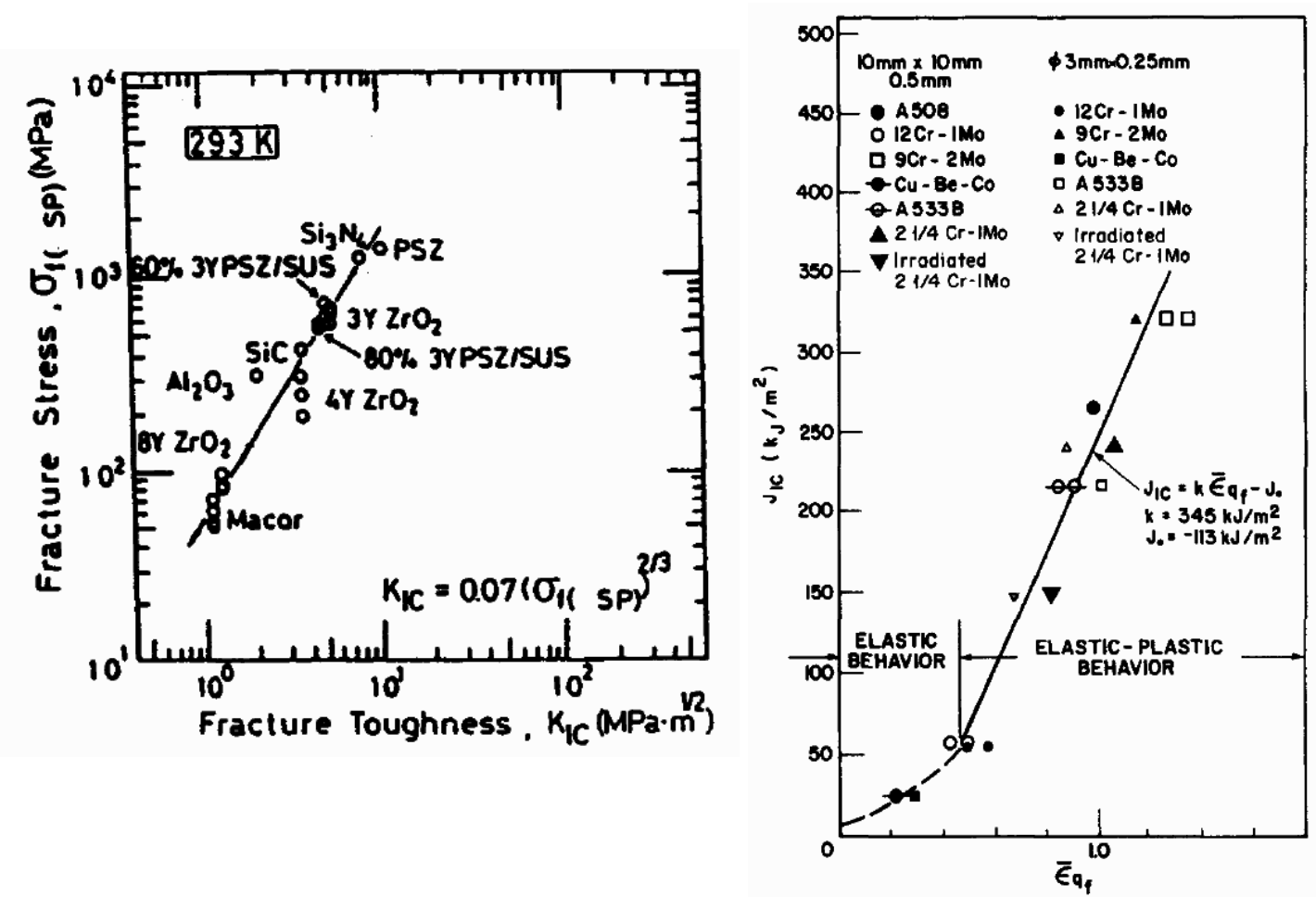

Figura 2.7. Relación entre $K_{I c} y \sigma_{f(S P)}$ [12] (izquierda); y entre $J_{I c}$ y $\bar{\varepsilon}_{q f}$ [14] (derecha) para varios materiales de la industria nuclear.
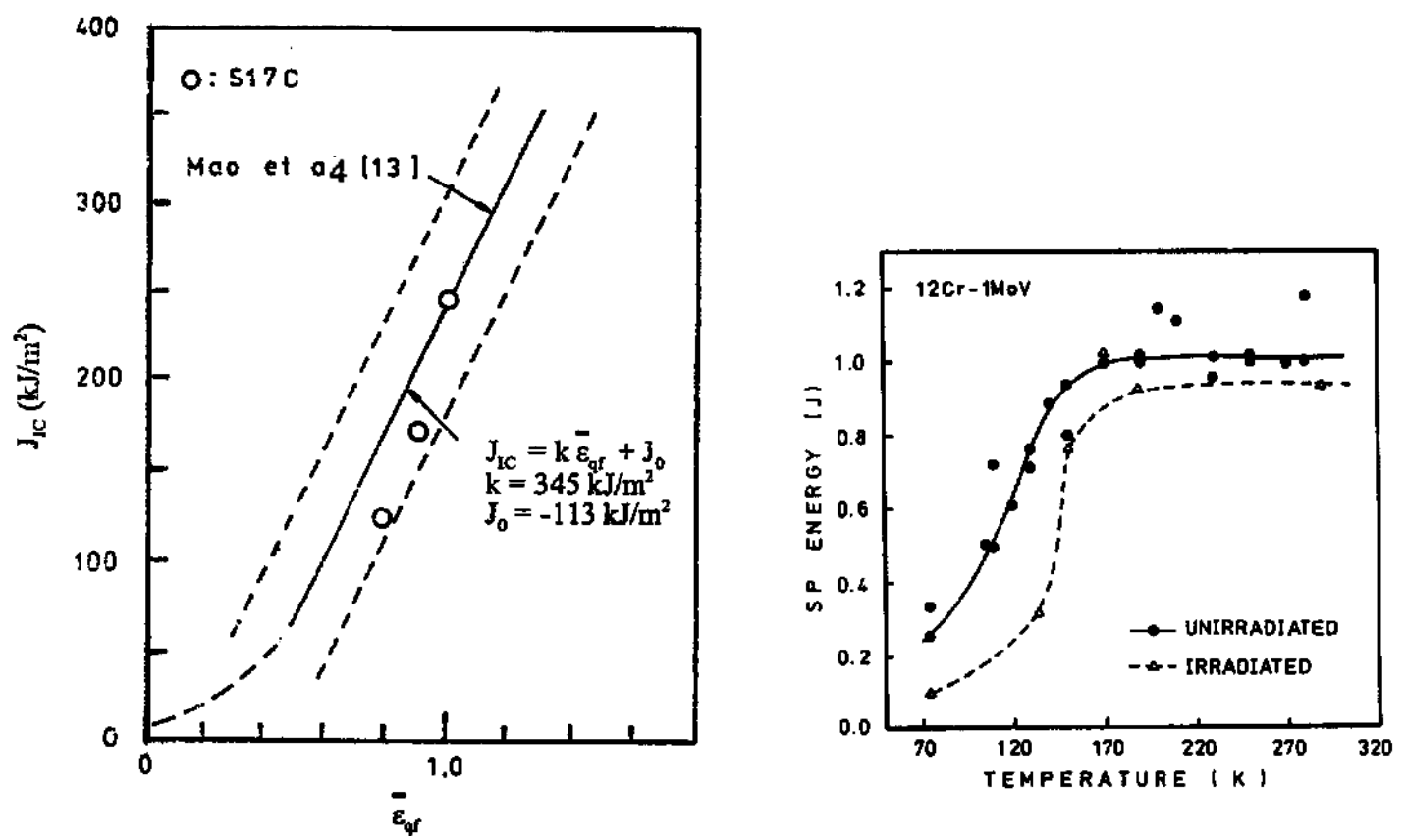

Figura 2.8. Relación entre $J_{I c}$ y $\overline{\mathcal{E}}_{q f}$ (izquierda) y dependencia de la temperatura con la energía absorbida en el SPT (derecha) [18]. 
En cualquiera de los trabajos anteriores se pone de manifiesto la relación lineal existente entre $J_{I c}$ y la $\overline{\mathcal{E}}_{q f}$ definida a partir de $\ln \left(t_{0} / t\right)$, por lo que se corroboran los resultados que obtuvieron los primeros investigadores en establecer dicha relación. Dicha relación se convierte en pilar fundamental de las líneas de investigación futuras que pretendan estimar las propiedades de fractura del material a partir del SPT.

En 1998 Fleury y Ha [19,20] realizaron una correlación entre las propiedades mecánicas y de fractura determinadas a partir del SPT y las obtenidas a partir de ensayos convencionales, como el ensayo de tracción uniaxial y el CVN, para aceros de baja aleación utilizados en centrales térmicas, como son el $12 \mathrm{Cr}-1 \mathrm{Mo}$, el $1 \mathrm{Cr}-0.5 \mathrm{Mo}$ y el $2.25 \mathrm{Cr}-1 \mathrm{Mo}$. Estos materiales fueron ensayados en un rango de temperatura de 25 a $600^{\circ} \mathrm{C}$.

Centrándonos en las propiedades de fractura, cabe destacar la correlación entre la tenacidad $K_{I c}$ del SPT y la tenacidad $K_{I c}$ del CVN que propusieron, la cuál se puede ver en la Figura 2.9. Los valores de $K_{I c}$ del SPT en el "lower shelf", es decir, cuando el comportamiento del material es frágil, fueron estimados a partir de las ecuaciones propuestas por Joo et al. [21] y los análisis SEM realizados. Mientras que los valores de $K_{I c}$ del SPT en el "upper shelf”, es decir, cuando el comportamiento del material es dúctil, fueron determinados a través de una ecuación basada en la energía del ensayo desarrollada por Afzal Khan et al. [22].

Se puede observar como los valores en el "upper shelf" tienen una mayor dispersión, aunque el valor medio ajusta adecuadamente con la relación lineal que propusieron. La correlación presentada por dichos autores es independiente de la temperatura de transición, lo cuál supone un gran avance ya que dicha temperatura, como se ha visto anteriormente, es diferente en los dos ensayos.

En ese mismo año Bulloch [23] hizo una recopilación de los resultados que hasta la fecha habían obtenido diversos investigadores de la temperatura de transición con el ensayo SPT y con el ensayo CVN. Esencialmente demostró algunas de las expresiones que ya existían en la literatura que relacionaban dichas temperaturas de transición. Este mismo autor había publicado anteriormente un estudio fractográfico del SPT [24]. Durante esta década, el ensayo SPT también fue utilizado en diversos campos de aplicación, como por ejemplo en el estudio de las propiedades de creep sobre aceros [25] o en medicina [26,27]. 


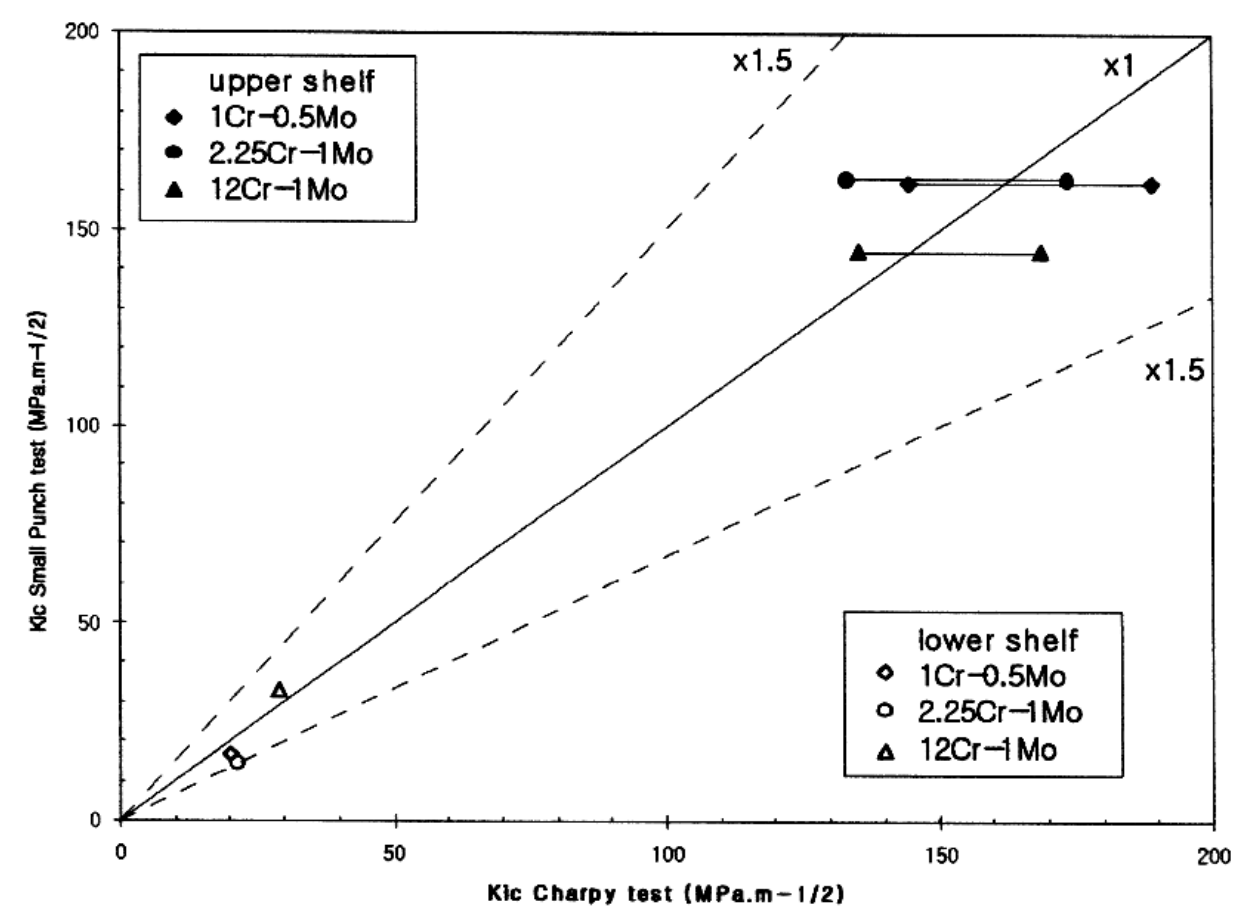

Figura 2.9. Correlación de $K_{I c}$ estimada en el SPT y en el CVN [20].

\subsection{3. Última década}

En el año 2001, Saucedo-Muñoz et al. [28] realizaron un trabajo de correlación entre el $J_{I c}$ y el $\overline{\mathcal{E}}_{q f}$ determinado a partir del SPT. Dicha correlación se llevó a cabo con aceros austeníticos envejecidos, aplicando la norma ASTM E813-89 [29] para la determinación de $J_{I c}$. La Figura 2.10 muestra la correlación obtenida, en comparación con la correlación propuesta por Misawa et al. [8] sobre aceros austeníticos, los cuales aplicaron la norma ASTM E813-81 [30] para la obtención de $J_{I c}$. De nuevo se pone de manifiesto la relación lineal existente entre $\bar{\varepsilon}_{q f}$ y $J_{I c}$, pudiendo observar en dicha figura como la correlación propuesta por Misawa et al. [8] no ajusta adecuadamente los resultados del estudio.

Comprobaron que la gran diferencia en la correlación era debida a la norma utilizada en la determinación de $J_{I c}$, a pesar de que la media del límite elástico de los materiales utilizados en este estudio era algo mayor que la de los materiales utilizados por Misawa et al. [8], $1160 M P a$ frente a $1020 M P a$. 


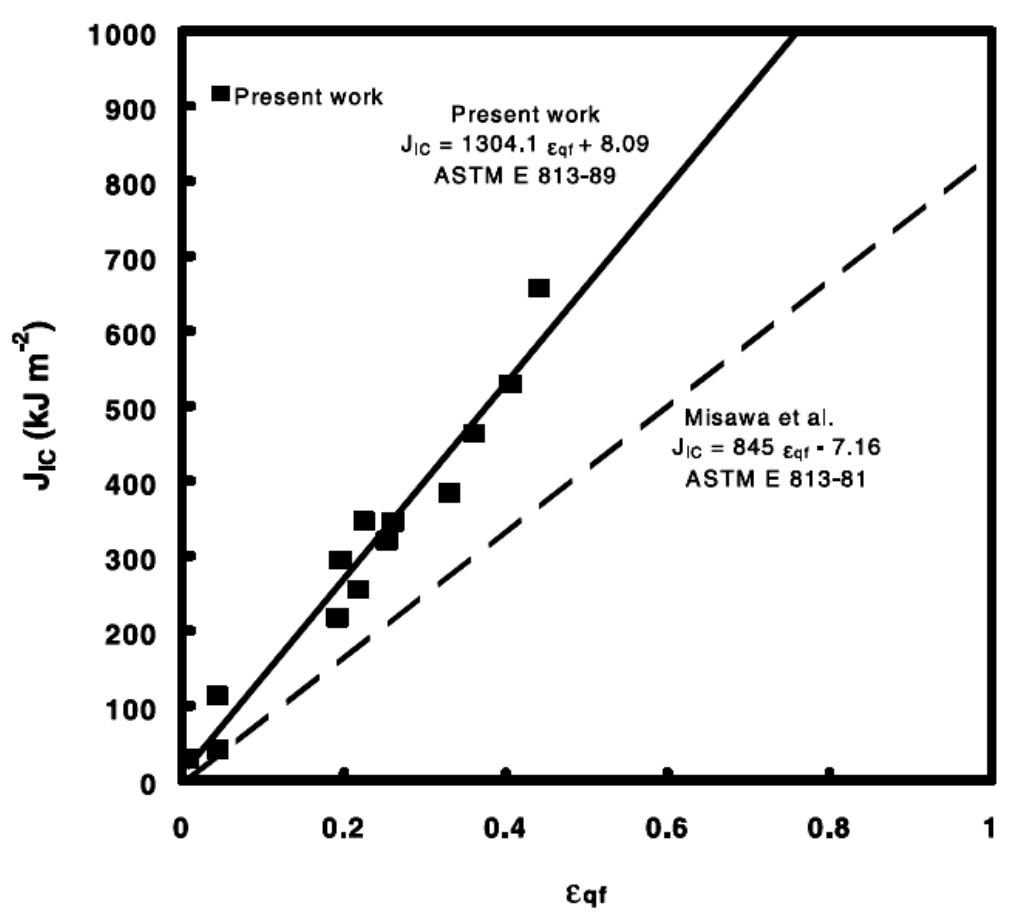

Figura 2.10. Regresiones lineales independientes de la temperatura entre $J_{I c}$ y $\overline{\mathcal{\varepsilon}}_{q f}$, realizadas por Saucedo-Muñoz et al. [28] y por Misawa et al. [8].

Cabe destacar, que la norma ASTM E813-81 define $J_{I c}$ como la intersección de la línea de enromamiento con el ajuste lineal de los puntos experimentales que tienen un incremento de grieta importante, mientras que el procedimiento descrito en la ASTM E813-89 define el valor de $J_{I c}$ como la intersección de la paralela a la línea de enromamiento trazada desde la abscisa $0.2 \mathrm{~mm}$ con el ajuste potencial de los datos $J-\Delta a$. Otras diferencias entre los procedimientos atañen al rango de crecimientos de grieta válidos. Así en la ASTM E813-81 se establece unos límites inferior y superior del $0.6 \%$ y $6 \%$ de la longitud de ligamento respectivamente. La ASTM E813-89 propone unos límites de exclusión situados en 0.15 y $1.5 \mathrm{~mm}$ de crecimiento de grieta. De esta forma, para poder comparar los resultados que obtuvieron con trabajos anteriores aplicaron la norma ASTM E813-81, proponiendo una nueva correlación entre $J_{I c}$ y $\overline{\mathcal{E}}_{q f}$ mostrada en la Figura 2.11, mejorando notablemente la similitud con la correlación propuesta por Misawa et al. [8]. En esta figura se puede ver también la correlación obtenida por Mao et al. [4] para diferentes aceros ferríticos y aleaciones no férreas, las cuales tienen un menor valor de $J_{I c}$, que implican un menor valor de la pendiente de la relación lineal. 
Como conclusiones interesantes de este trabajo se pueden destacar la importancia del procedimiento seguido para la determinación de $J_{I c}$ y la confirmación, una vez más, de la independencia con la temperatura de ensayo que presenta este tipo de correlaciones lineales entre $J_{I c}$ y $\bar{\varepsilon}_{q f}$.

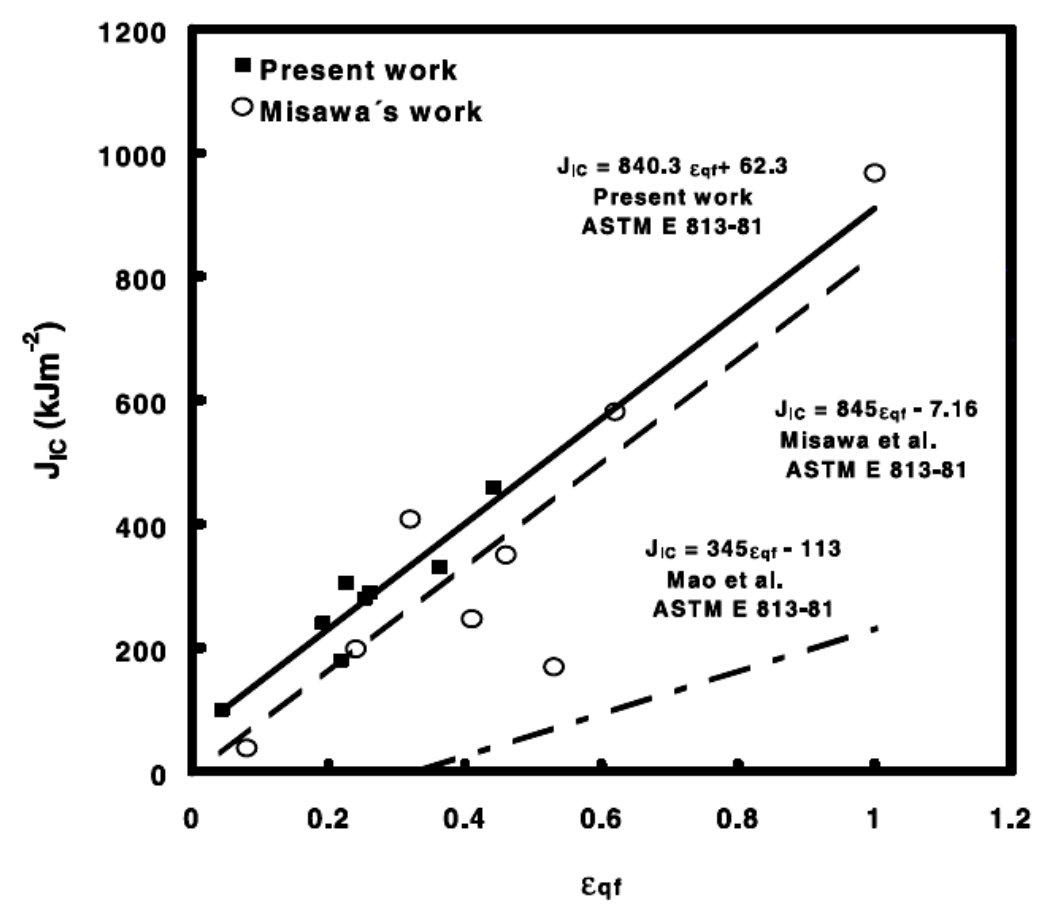

Figura 2.11. Regresiones lineales independientes de la temperatura entre $J_{I c}$ y $\overline{\mathcal{E}}_{q f}$, realizadas por Saucedo-Muñoz et al. [28], por Misawa et al. [8] y por Mao et al. [4].

Un año más tarde, Shekhter et al. [31] desarrollaron un estudio para la determinación de $K_{I c}$ en una pieza del material $1 \mathrm{Cr}-1 \mathrm{Mo}-0.25 \mathrm{~V}$ sometida a diferentes tratamientos térmicos. Utilizaron tres métodos para medir el $K_{I c}$ : el ensayo CVN, el SPT y el ensayo de fractura. Para estimar el valor de $K_{I c}$ a partir del CVN y del SPT propusieron la utilización de diferentes correlaciones empíricas disponibles en la bibliografía, las cuáles recogen en su trabajo. Comprobaron que los valores de $K_{I c}$ estimados de esta forma eran comparables con los valores medidos experimentalmente de $K_{I c}$ en el ensayo de fractura. Basándose en la correlación de dichos resultados, concluyeron que el SPT era un método válido para la estimación de $K_{I c}$. 
También en el año 2002 Ruan et al. [32] utilizaron el SPT para evaluar las propiedades de un acero martensítico EUROFER97 tratado térmicamente. Establecieron una serie de correlaciones de la carga $P_{y}$ y $P_{\max }$ en el SPT con la tensión de fluencia y la tensión última en el ensayo de tracción respectivamente, siguiendo la misma metodología propuesta por Mao y Takahashi [4]. Dichas correlaciones se pueden ver en la Figura 2.12, quedando definidas por las expresiones (2.7) y (2.8) respectivamente. En dicha figura se puede comprobar el buen ajuste de los resultados con las correlaciones propuestas por estos autores, evidenciando de nuevo la relación lineal existente entre los parámetros estudiados.

$$
\begin{aligned}
& \sigma_{0.2}[\mathrm{MPa}]=(149 \pm 108)+(413 \pm 68) \cdot \frac{P_{y}}{t_{0}^{2}}\left[\mathrm{kN} / \mathrm{mm}^{2}\right] \\
& \sigma_{u t s}[\mathrm{MPa}]=(218 \pm 101)+(77 \pm 15) \cdot \frac{P_{\max }}{t_{0}^{2}}\left[\mathrm{kN} / \mathrm{mm}^{2}\right]
\end{aligned}
$$

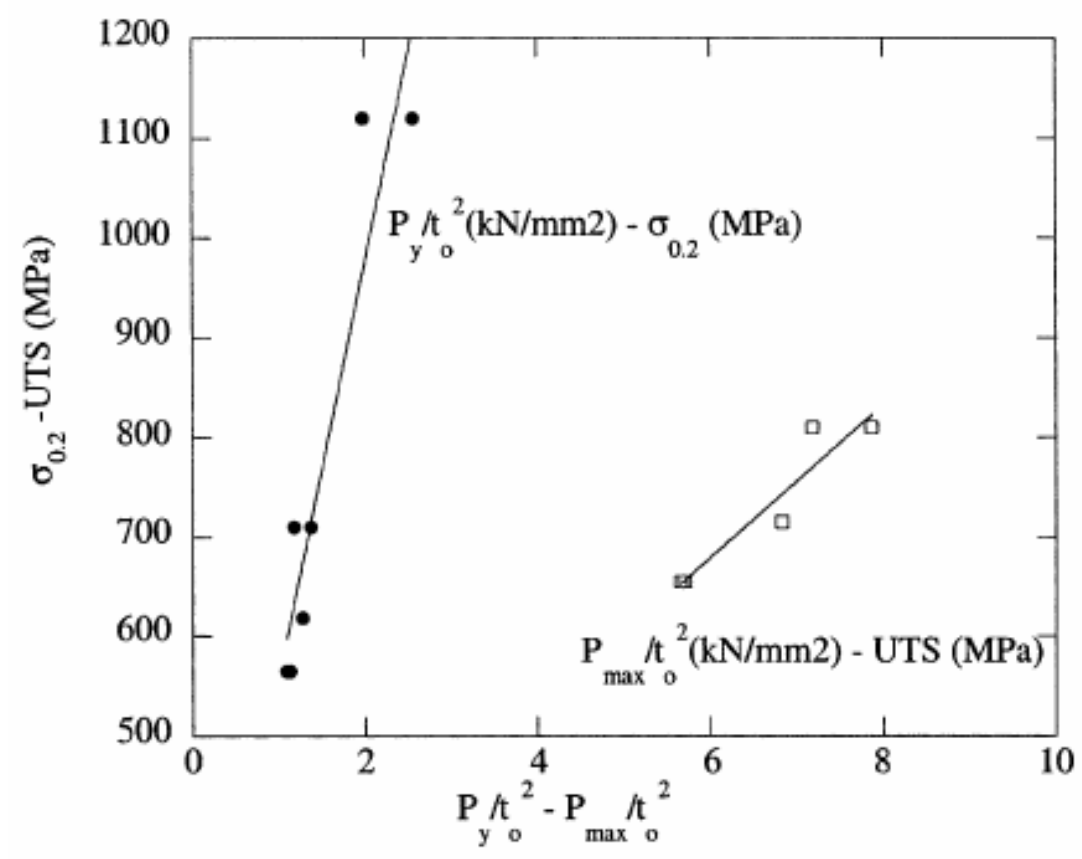

Figura 2.12. Correlaciones obtenidas por Ruan et al. [32].

Se puede destacar, que todos los trabajos analizados hasta el momento han sido realizados utilizando probetas SPT, cuadradas o circulares, sin mecanizarlas ningún tipo de grieta, consiguiendo en mayor o menor medida una buena estimación de las propiedades a fractura del material. Dichas propiedades se determinan convencionalmente con ensayos que utilizan 
probetas que tienen mecanizada una grieta, como por ejemplo la probeta Charpy en el ensayo CVN o la probeta compacta (CT) en el ensayo de fractura. Por lo que la posibilidad de utilizar probetas SPT con grieta para la determinación de las propiedades a fractura del material, podría suponer una buena alternativa con la que mejorar los resultados obtenidos hasta la fecha. En este sentido, en el año 2003 Ju et al. [33] realizaron un estudio pionero de SPT utilizando probetas $10 \times 10 \times 0.5 \mathrm{~mm}$ con grieta para obtener las propiedades a fractura del acero ASME SA 508 Clase 3, utilizado en Korea en vasijas de reactores nucleares. La grieta pasante se mecanizaba en el centro de la probeta, en la Figura 2.13 se puede observar el detalle de la zona central de la probeta donde está ubicada dicha grieta, la cuál tiene forma elíptica, así como el fallo frágil a baja temperatura iniciado en esa zona. En función de parámetros geométricos $\left(a_{1}, r_{1}, c_{1}\right.$ y $\left.h_{1}\right)$ y de la carga en el momento de inicio de la fisura $\left(P_{i}\right)$, desarrollaron una fórmula para obtener la tenacidad a fractura $\left(K_{C}\right)$, definida por la expresión (2.9).

$K_{C}=\frac{3}{2} \cdot \frac{P_{i} \cdot(1+v)}{\pi \cdot h_{1}^{2}} \cdot\left(\ln \frac{c_{1}}{b_{1}}+\frac{r_{1}^{2}}{4 \cdot c_{1}^{2}}\right) \cdot \sqrt{a_{1}}$

Donde $a_{1}$ es el semieje mayor de la elipse a la que se puede asemejar la grieta, $r_{1}$ es el radio de contacto con el punzón, $c_{1}$ es el radio de la matriz inferior, $h_{1}$ es el lado de la probeta y $v$ es el coeficiente de Poisson. El inicio de la fisura se determinó utilizando señales de emisión acústica, pudiendo de esta manera determinar el valor de $P_{i}$ en la curva carga-desplazamiento del SPT. La tenacidad a fractura estimada a partir de la expresión desarrollada, la compararon con los valores provenientes de la "master curve" propuesta en la norma ASTM E1921 [34], obteniendo unos resultados congruentes en la región "lower shelf". Con estos resultados demostraron la posibilidad de extraer las propiedades a fractura del material analizado a través del SPT, haciendo uso de probetas SPT con una grieta mecanizada inicialmente.

Un año más tarde, en el 2004 Finarelli et al. [35] investigaron sobre las propiedades mecánicas a partir del SPT en materiales irradiados, los materiales utilizados fueron el acero austenítico 316L y el acero martensítico Optifer. La Figura 2.14 muestra las curvas cargadesplazamiento del SPT obtenidas con diferentes dosis de irradiación en los materiales utilizados. 

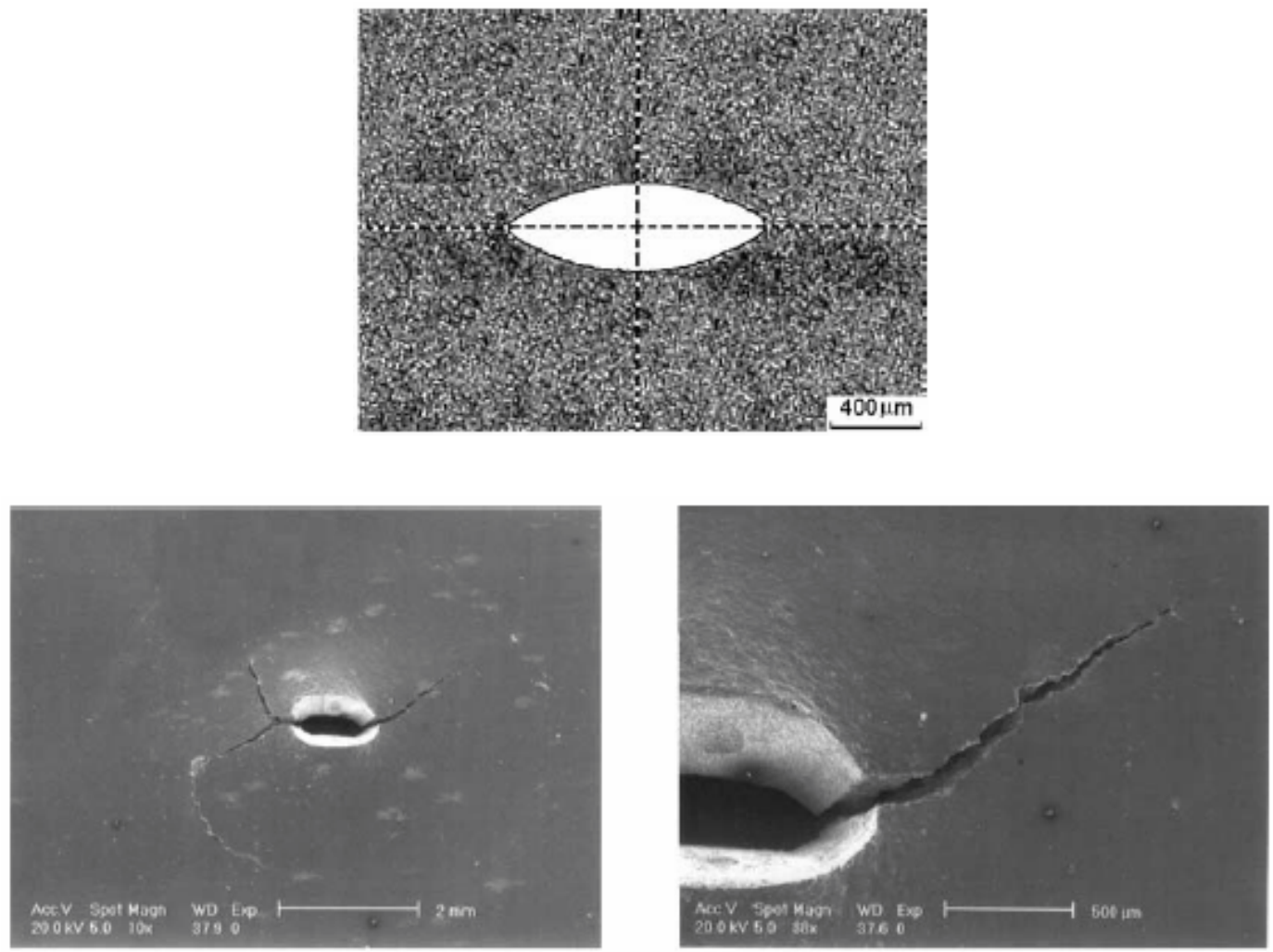

Figura 2.13. Fisura y micrografías del fallo frágil de la probeta utilizada por Ju et al. [33].

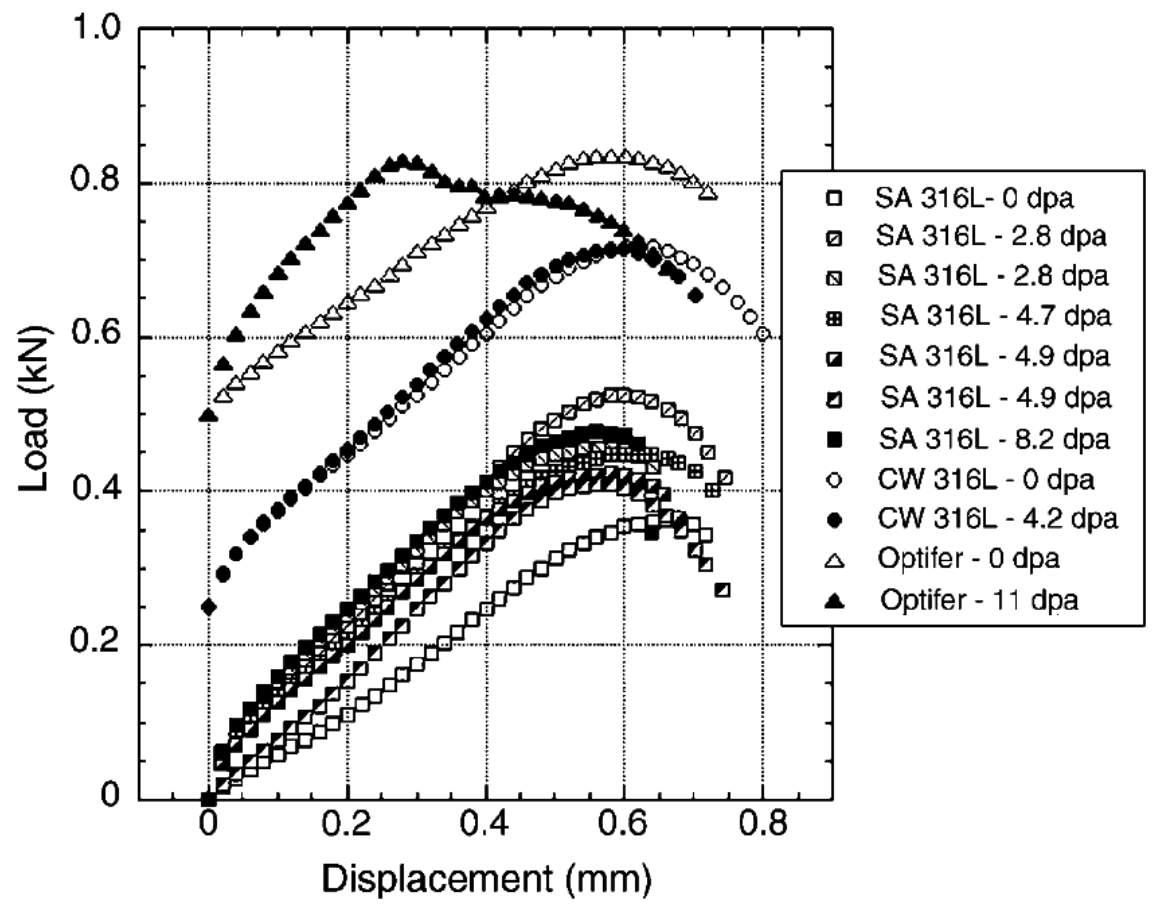

Figura 2.14. Curvas carga-desplazamiento de SPT obtenidas para diferentes materiales y dosis de irradiación [35]. 
Con los datos obtenidos en los ensayos fueron capaces de determinar la tensión de fluencia $\sigma_{y}$ y la deformación equivalente de fractura $\bar{\varepsilon}_{q f}$, utilizando las expresiones recogidas anteriormente propuestas por Mao et al. [4], y la energía de fractura, calculada como el área debajo de la curva carga-desplazamiento del ensayo SPT desde el inicio hasta el punto de carga máxima, de cada material y para cada dosis de irradiación.

En ese mismo año Campitelli et al. [36] utilizaron el SPT y la simulación numérica para determinar las propiedades mecánicas $\left(\begin{array}{lll}\sigma_{y} & \text { y } & \sigma_{u t s}\end{array}\right)$ del acero austenítico $316 \mathrm{~L}$ y del acero martensítico F82H, a través de las expresiones propuestas por Mao et al. [4]. Su investigación se centró principalmente en determinar la influencia del espesor de la probeta y del coeficiente de fricción en los resultados obtenidos. Las principales conclusiones a las que llegaron fueron que para el caso de $\sigma_{y}$ sí que existía una correlación con $P_{y} / t_{0}^{2}$, pero para el caso de $\sigma_{u t s}$ no fueron capaces de obtener una correlación adecuada con $P_{\max } / t_{0}^{2}$, como la que obtuvieron Mao et al. [4]. Además de esto, concluyeron que la implementación del coeficiente de fricción en la simulación numérica era fundamental para la correcta reproducibilidad de la curva carga-desplazamiento experimental. A estas mismas conclusiones, llegaron dichos autores en un trabajo posterior [37] realizado sobre probetas irradiadas de acero martensítico.

La mayoría de los trabajos anteriores, especialmente los de la década de los 80, representan la base fundamental en la que se apoyan las investigaciones realizadas durante estos últimos años, en lo que a la obtención, a través del SPT, de las propiedades a fractura se refiere. Ciñéndose la inmensa mayoría de estos estudios a la estimación de algún parámetro de fractura, a través de expresiones disponibles en la bibliografía, para un material en concreto, principalmente aceros. En este sentido, a modo de resumen se pueden comentar los trabajos llevados a cabo por Eskner et al. [38], Callaghan et al. [39], Komazaki et al. [40], Dunne et al. [41], Nakata et al. [42], Budzakoska et al. [43], Hyde et al. [44], Kim et al. [45], Sunjaya et al. [46], Wang et al. [47]. Destacar también, que han sido muy prolíferos en esta última década los estudios sobre el SPT para otras aplicaciones, como por ejemplo los llevados a cabo para evaluar la DBTT, por Cheon et al. [48], Song et al. [49,50], Jia et al. [51], Kim et al. [52], Serre et al. [53], o para determinar propiedades de creep, por Dobes et al. [54-56], Yang et al. [57], Zhai et al. [58,59], Sugimoto et al. [60], Chen et al. [61], Baek et al. [62], Nakata et al. [63] y Komazaki et al. [64]. 


\subsection{PROCEDIMIENTOS DE INTEGRIDAD ESTRUCTURAL}

Los procedimientos de integridad estructural se están imponiendo como una excelente herramienta de evaluación de componentes fisurados. Desde mediados de los años 70 han surgido diferentes procedimientos para evaluar el estado de componentes en servicio en diferentes sectores de aplicación. Estos procedimientos son capaces de determinar si el fallo del componente se produce por colapso plástico, por fractura frágil o por una mezcla de las dos anteriores. En general, dos son las elecciones básicas que el analista debe tener en cuenta a la hora de la aplicación de un procedimiento. Primero, la aproximación al problema o como se trata el modo de fallo, y segundo el nivel de análisis, en función de la información disponible del componente y material, así como de los conocimientos para efectuar el análisis.

Actualmente se plantean dos tipos de aproximaciones al problema de la evaluación de la integridad estructural de un componente fisurado. El primero utiliza el concepto del diagrama de fallo o Failure Assessment Diagram (FAD) como herramienta de análisis, y el segundo utiliza una aproximación en base a consideraciones energéticas a través del diagrama de la fuerza motriz de agrietamiento o Crack Driving Force (CDF). Ambas aproximaciones están basadas en el mismo principio científico, que postula que el fallo del componente fisurado se alcanza cuando se le somete a una carga límite, definida mediante una combinación de criterios de mecánica de fractura y de plasticidad. El análisis según la mecánica de fractura realiza una comparación entre el nivel tensional en la punta de la fisura (a través del factor de intensidad de tensiones) y la capacidad del material para oponerse a la rotura (denominado tenacidad a fractura del material o resistencia a la fractura). El nivel tensional en la punta de la fisura debe ser evaluado, en la mayoría de los casos, utilizando conceptos elasto-plásticos y considerando su dependencia con la geometría del componente, tamaño y forma de la fisura, propiedades del material y cargas aplicadas.

En la aproximación mediante el diagrama FAD, ambas comparaciones, el estado tensional con la tenacidad a fractura del material y la carga aplicada con respecto a la carga de colapso plástico, se conjugan al mismo tiempo. Por su parte, en la aproximación mediante la carga límite, CDF, la fuerza motriz aplicada es dibujada y comparada directamente con la tenacidad a fractura del material. Un análisis desacoplado se efectúa para el análisis de la carga de colapso plástico. 
La elección de una u otra aproximación queda a decisión del evaluador, donde una aproximación no presenta ventajas o diferencias con respecto a la otra. Teniendo en cuenta que, en general, el análisis proporcionado por ambas aproximaciones es conservativo, tanto más cuanto menor sea el nivel de análisis elegido, la carga de fallo viene sobredimensionada, lo cuál indica que generalmente el valor utilizado de la tenacidad del material estará del lado de la seguridad. Este es un hecho importante en el desarrollo de la presente Tesis, dado que el objetivo de la misma es estimar el valor de la tenacidad del material utilizando el diagrama FAD de una geometría fisurada. En consecuencia es de esperar que el valor obtenido con la metodología presentada muestre un cierto grado de conservadurismo frente a la tenacidad real del material.

Con el objetivo de familiarizarnos con los diferentes procedimientos de integridad estructural, y con la idea de remarcar que todos ellos se basan en las mismas suposiciones, se presenta en los siguientes párrafos una recopilación de los mismos. Una clasificación típica, atendiendo a la recogida habitualmente en la bibliografía [65,66], hace referencia al modo de aproximación al fallo que realizan. Algunos primeros estudios pueden encontrarse en la literatura, como los orígenes de los procedimientos de integridad estructural, como el desarrollado por Battelle [67-69] para analizar el caso de tuberías con grietas circunferenciales y determinar si el componente analizado será capaz de alcanzar la carga de colapso plástico, o el procedimiento KWU desarrollado por Golembiewski y Vasoukis [70,71] a mediados de la década de los 80, a partir de la realización de numerosos ensayos Charpy sobre componentes de la industria nuclear, especialmente vasijas a presión y tuberías. Este método establece básicamente que cuando se alcanza el valor umbral de $45 \mathrm{~J}$ en el ensayo Charpy se produce el colapso plástico del componente.

\subsubsection{Aproximación mediante el Diagrama de Fallo, FAD}

Estos procedimientos utilizan el Diagrama de Evaluación de Fallo (Failure Assessment Diagram) como eje del análisis, permitiendo en muchos casos una evaluación rápida y precisa del componente analizado, y teniendo en cuenta tanto el fallo por fractura frágil como el colapso plástico. El diagrama de fallo o FAD, es una representación de la envolvente del lugar del fallo de una estructura fisurada, definido en términos de dos parámetros, $K_{r}$ y $L_{r}$. El 
valor del ratio de tenacidad $K_{r}$, se puede definir en primera instancia como el cociente entre el factor de intensidad de tensiones aplicado $\left(K_{I}\right)$ y la tenacidad a fractura del material $\left(K_{\text {mat }}\right)$, es decir,

$$
K_{r}=\frac{K_{I}}{K_{m a t}}
$$

Por su parte, el valor del ratio de carga $L_{r}$ es el cociente entre la tensión característica aplicada al componente, o tensión de referencia, y la tensión que causa el colapso plástico de la estructura fisurada. Dicho cociente puede expresarse también en función de las cargas aplicadas y la carga de colapso plástico del componente.

$$
L_{r}=\frac{\sigma_{r e f}}{\sigma_{y}}=\frac{P_{a p l}}{P_{U}}
$$

Donde $P_{a p l}$ es la suma de las cargas principales aplicadas, las cuáles contribuyen a la plastificación del componente fisurado, y $P_{U}$ es el valor de la carga de colapso plástico del mismo. El valor de $P_{U}$ es complejo de calcular ya que depende de las propiedades del material y de la geometría del componente fisurado. Para la determinación de este valor se pueden usar varios procedimientos como la utilización de expresiones analíticas recogidas en la bibliografía [72], la simulación numérica o el análisis empírico de un modelo realizado a escala. La línea de fallo, en consecuencia, viene definida mediante una curva del tipo:

$$
K_{r}=f\left(L_{r}\right)
$$

En la Figura 2.15 aparece un esquema del diagrama de fallo FAD, donde en general se incorpora un valor límite para el ratio $L_{r}=L_{r}^{\max }$ que define el límite del colapso plástico de la estructura fisurada. La expresión universalmente asumida para este límite es:

$$
L_{r}^{\max }=\frac{1}{2} \cdot \frac{\left(\sigma_{0.2}+\sigma_{u t s}\right)}{\sigma_{0.2}}
$$

donde $\sigma_{0.2}$ es el límite elástico del material para una deformación plástica del $0.2 \%$, y $\sigma_{u t s}$ es la tensión última a tracción. 


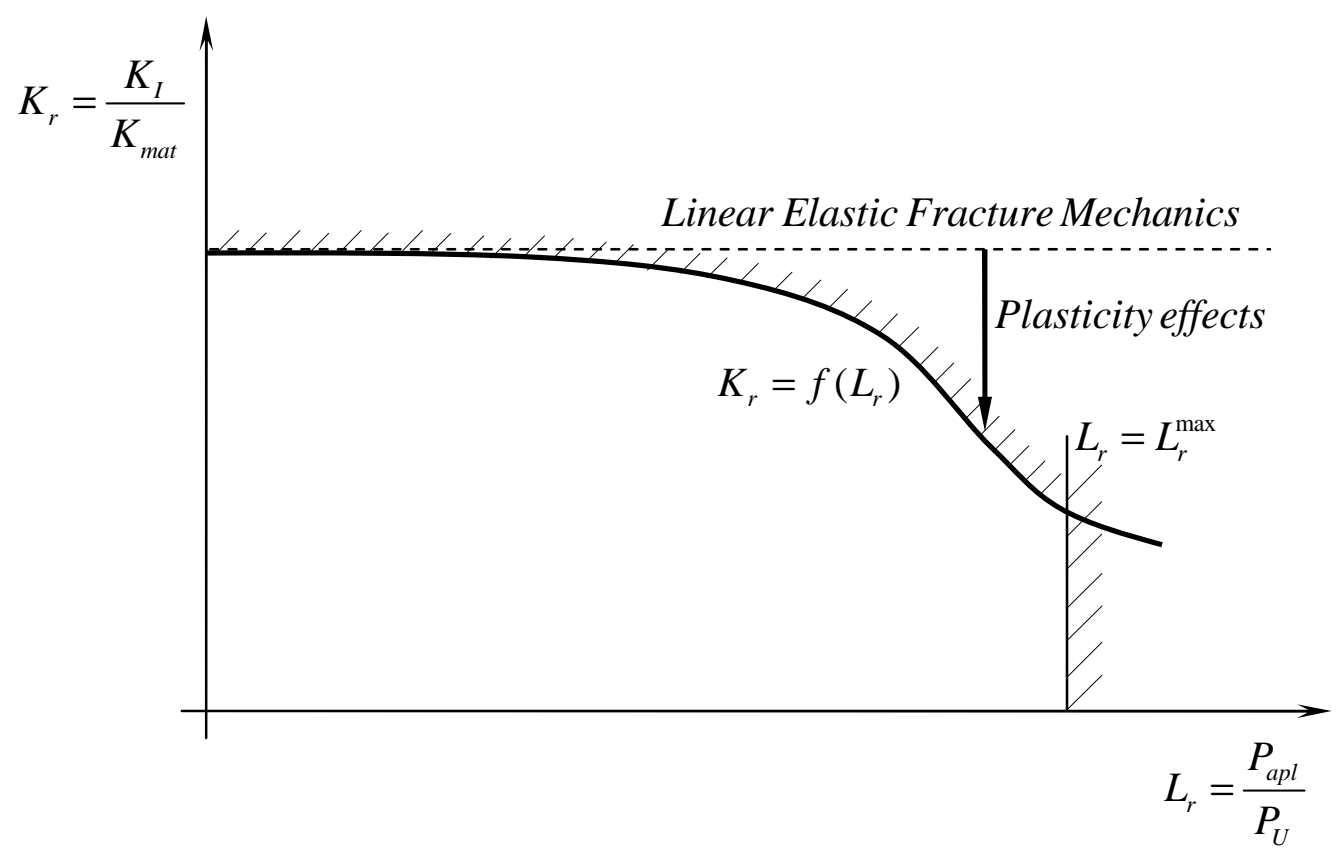

Figura 2.15. Esquema del diagrama de fallo FAD.

Los numerosos procedimientos de integridad estructural existentes proporcionan diferentes niveles de análisis, encaminados a la obtención del diagrama FAD del componente fisurado. En general, se puede hablar de tres niveles de análisis en función de la cantidad de información disponible del material y del componente fisurado. En los siguientes párrafos se presentan los niveles u opciones de análisis contemplados por los procedimientos de uso más extendido.

\subsubsection{Opción 1: Curva general}

Esta opción es apropiada para todos los materiales que no tienen una zona de fluencia en la curva tensión-deformación y queda definida por la expresión (2.14). Para su aplicación sólo es necesario conocer $\sigma_{0.2}$ y $\sigma_{u t s}$ para poder determinar la línea de fallo, por lo que es considerada como la más sencilla de aplicar.

$$
\begin{array}{lll}
K_{r}=\left(1-0.14 \cdot L_{r}^{2}\right)\left[0.3+0.7 \cdot \exp \left(-0.65 \cdot L_{r}^{6}\right)\right] & \text { si } & L_{r} \leq L_{r}^{\max } \\
K_{r}=0 & \text { si } & L_{r}>L_{r}^{\max }
\end{array}
$$


La Figura 2.16 muestra la representación de la ecuación (2.14), así como alguna de las líneas típicas de corte en algunos aceros. Esta opción subestima razonablemente las fisuras en un componente. Sin embargo, en algunos casos, el grado de subestimación puede ser excesivo, por lo que se recomienda utilizar la Opción 2, con el inconveniente de tener que conocer los datos de la curva tensión-deformación. Para los materiales que presentan una zona de fluencia, el uso de la Opción 1 se restringe a valores de $L_{r}<1.0$ por lo que es preferible hacer uso de la Opción 2. Esta opción es la utilizada en la Opción 1 del R6, en el Nivel 2A del BS 7910 y en el Nivel 2 del API 579.

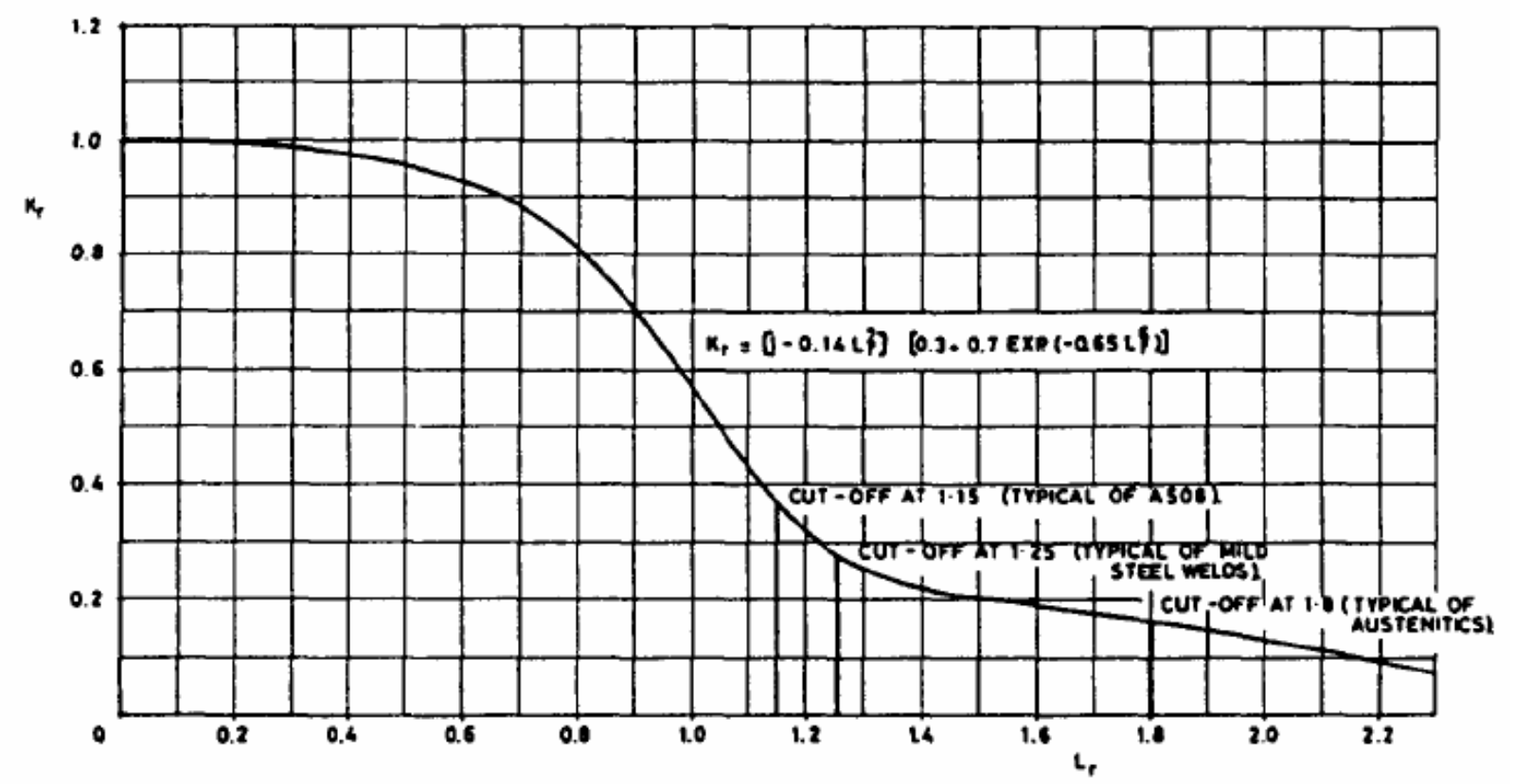

Figura 2.16. Representación de la curva que define la Opción 1 del FAD [72], junto con diferentes líneas de corte en función del material analizado.

\subsubsection{Opción 2: Curva específica del material}

Esta opción proporciona un grado de exactitud mayor que la anterior, particularmente en aquellos casos en los que los materiales presentan una zona de fluencia, quedando definida por la expresión (2.15).

$$
\begin{array}{lll}
K_{r}=\left(\frac{E \cdot \varepsilon_{r e f}}{L_{r} \cdot \sigma_{0.2}}+\frac{L_{r}^{3} \cdot \sigma_{0.2}}{2 \cdot E \cdot \varepsilon_{r e f}}\right)^{-1 / 2} & \text { si } & L_{r} \leq L_{r}^{\max } \\
K_{r}=0 & \text { si } & L_{r}>L_{r}^{\max }
\end{array}
$$


Donde $E$ es el módulo de Young y $\varepsilon_{r e f}$ es la deformación verdadera para la tensión $L_{r} \cdot \sigma_{0.2}$. Cuando $L_{r}=1, \varepsilon_{\text {ref }}=\sigma_{0.2} / E+0.002$. Dicha opción tiene una línea de corte en la zona de colapso plástico definida mediante la expresión (2.13). Para poder aplicar este nivel es necesario conocer la curva completa tensión-deformación del material, la cuál es determinada a partir de ensayos convencionales de tracción. En particular es necesario disponer de los datos de la curva de tracción en valores $\sigma_{r e f} / \sigma_{0.2}=0.7,0.8,0.98,1.0,1.02,1.1,1.2 \mathrm{y}$ en intervalos de 0.1 hasta alcanzar la tensión última $\sigma_{u t s}$. Este aspecto dependerá de la forma de la curva de tracción. Esta opción es la utilizada en la Opción 2 del R6, en el Nivel 2B del BS 7910 y en el Nivel 3B del API 579.

\subsubsection{Opción 3: Curva obtenida usando un análisis de la integral J}

La curva utilizada en esta opción es específica para cada material y geometría, la cuál se obtiene a partir de la siguiente expresión,

$$
\begin{array}{lll}
K_{r}=\left(\frac{J_{e}}{J_{a p p}}\right)^{1 / 2} & \text { si } & L_{r} \leq L_{r}^{\max } \\
K_{r}=0 & \text { si } & L_{r}>L_{r}^{\max }
\end{array}
$$

Donde $J_{a p p}$ es la integral $J$ aplicada y $J_{e}$ su parte elástica para un mismo valor de carga, es decir, para el mismo valor de $L_{r}$, así $K_{r}$ queda representado en función de $L_{r}$. Al igual que en las dos opciones anteriores la línea de corte en la zona de colapso plástico queda definida por la expresión (2.13). Esta opción requiere la evaluación de la integral $J$ en la estructura fisurada bajo las condiciones de carga existentes. Todos los análisis para evaluar $J_{a p p}$ y $J_{e}$ deben ser realizados usando códigos computacionales válidos. En estos análisis se debe usar una curva tensión-deformación bien definida para bajas deformaciones. Esta opción es la utilizada en la Opción 3 del R6, en el Nivel 3 del BS 7910 y en el Nivel 3C del API 579.

La Figura 2.17 muestra la representación conjunta de las tres posibles opciones del FAD, se puede observar como la Opción 1 es con la que se obtienen los resultados más conservadores. Por otro lado, conforme se pasa de la Opción 1 a la 3 la zona segura del diagrama aumenta, proporcionando de esta manera resultados con un grado de exactitud mayor. 


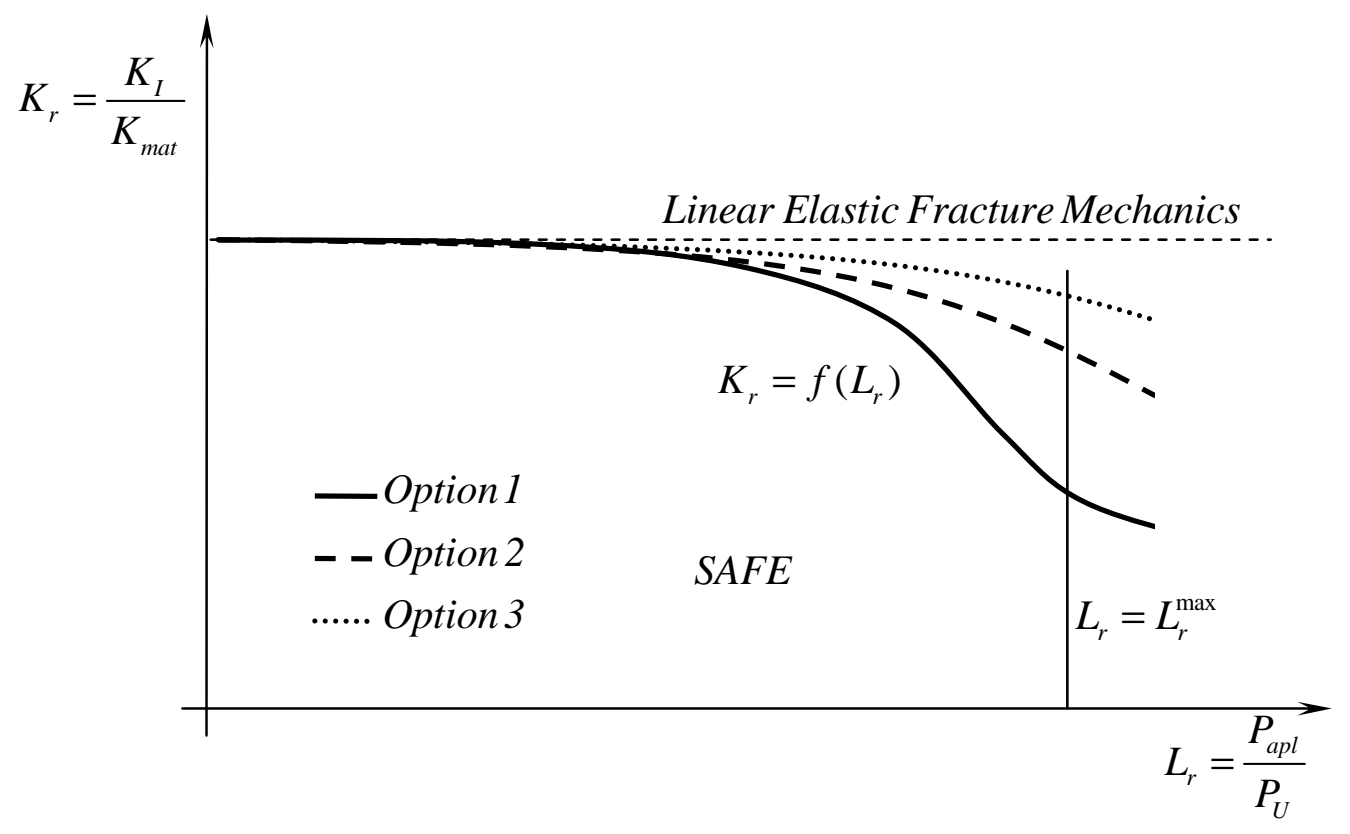

Figura 2.17. Esquema del diagrama de fallo FAD para los diferentes niveles de análisis.

\subsubsection{Categorías de análisis}

Un aspecto a tener en cuenta para la aplicación del diagrama FAD es la posibilidad de elegir entre tres categorías de análisis. Este concepto hace referencia a la posibilidad de elegir entre tres diferentes criterios para el valor de la tenacidad del material. La Categoría 1 es la más simple, y en ella la iniciación de la fisuración viene definida por la tenacidad a fractura de iniciación. Su elección es apropiada cuando el fallo se produce por mecanismos de fractura frágil en ausencia de desgarro dúctil inicial o cuando se produce por mecanismos de fractura dúctil y no aporta ningún beneficio tener en cuenta el desgarro dúctil.

En el primer caso se usa la tenacidad a fractura en deformación plana elástica lineal en el inicio de la rotura frágil, comprobando los requisitos de validez que establecen las normas [72]. Si se cumplen a la tenacidad a fractura se la denomina $K_{I c}$ y si no se cumplen $K_{c}$. En el segundo caso se usa la tenacidad a fractura después de un enromamiento de la punta de fisura de $0.2 \mathrm{~mm}$ y del inicio de propagación de la fisura $\left(K_{0.2}\right)$. Este valor de tenacidad proporciona una buena aproximación ingenieril de la tenacidad a fractura para su uso en evaluaciones estructurales basadas en el inicio de fisura. 
Las Categorías 2 y 3 permiten proporcionar una descripción más detallada del comportamiento de estructuras dúctiles incluyendo el fenómeno del desgarro dúctil en la evaluación del componente. La Categoría 2 es evaluada a través de un nivel de tenacidad a fractura, $K_{g}$, definido para un pequeño valor de desgarro dúctil. El valor de $K_{g}$ es la máxima tenacidad a la que se considera que la integral J caracteriza el comportamiento a fractura del material según los criterios de validez establecidos por el procedimiento en cuestión [72]. La Categoría 3 es la más compleja de aplicar y la que ofrece un grado de exactitud mayor, dado que la propagación de la fisura es evaluada a través de la curva de desgarro dúctil, la cuál proporciona un valor de tenacidad a fractura para un determinado valor de desgarro, $K_{\Omega}$.

En consecuencia, el ratio de tenacidad $K_{r}$ puede ser evaluado, dependiendo de la categoría de análisis elegida, a través de la siguiente expresión:

$K_{r}= \begin{cases}K_{I}(a) / K_{m a t} & \text { Categoría } 1 \\ K_{I}(a) / K_{g} & \text { Categoría } 2 \\ K_{I}(a) / K_{\Omega} & \text { Categoría } 3\end{cases}$

Donde $K_{I}(a)$ es el factor de intensidad de tensiones elástico lineal de las cargas principales aplicadas en el componente para un determinado valor de la longitud de fisura $a$, y $K_{\text {mat }}$ es la tenacidad a fractura del material, donde en la Categoría 1 el valor de $K_{m a t}$ corresponde según proceda con el valor de $K_{I c}, K_{c}$ o $K_{0.2}$ y en el caso de las Categorías 2 y 3 corresponde con el valor de $K_{0.2}$.

Un esquema típico para la aplicación de los procedimientos de integridad estructural aparece representado en la Figura 2.18, que puede encontrarse en el trabajo de Milne [72]. Según este esquema resulta aconsejable, desde el punto de vista de la evaluación de un componente fisurado, comenzar por las categorías de análisis más sencillas, y el diagrama FAD más general, que proporcionan una evaluación más conservadora del componente.

En caso de no cumplirse la evaluación se procede a refinar el análisis en varios frentes: utilizar una categoría superior de análisis, utilizar una caracterización de la fisura menos conservadora, utilizar un diagrama FAD obtenido con un nivel de análisis más elevado o efectuar un análisis tensional más refinado. 


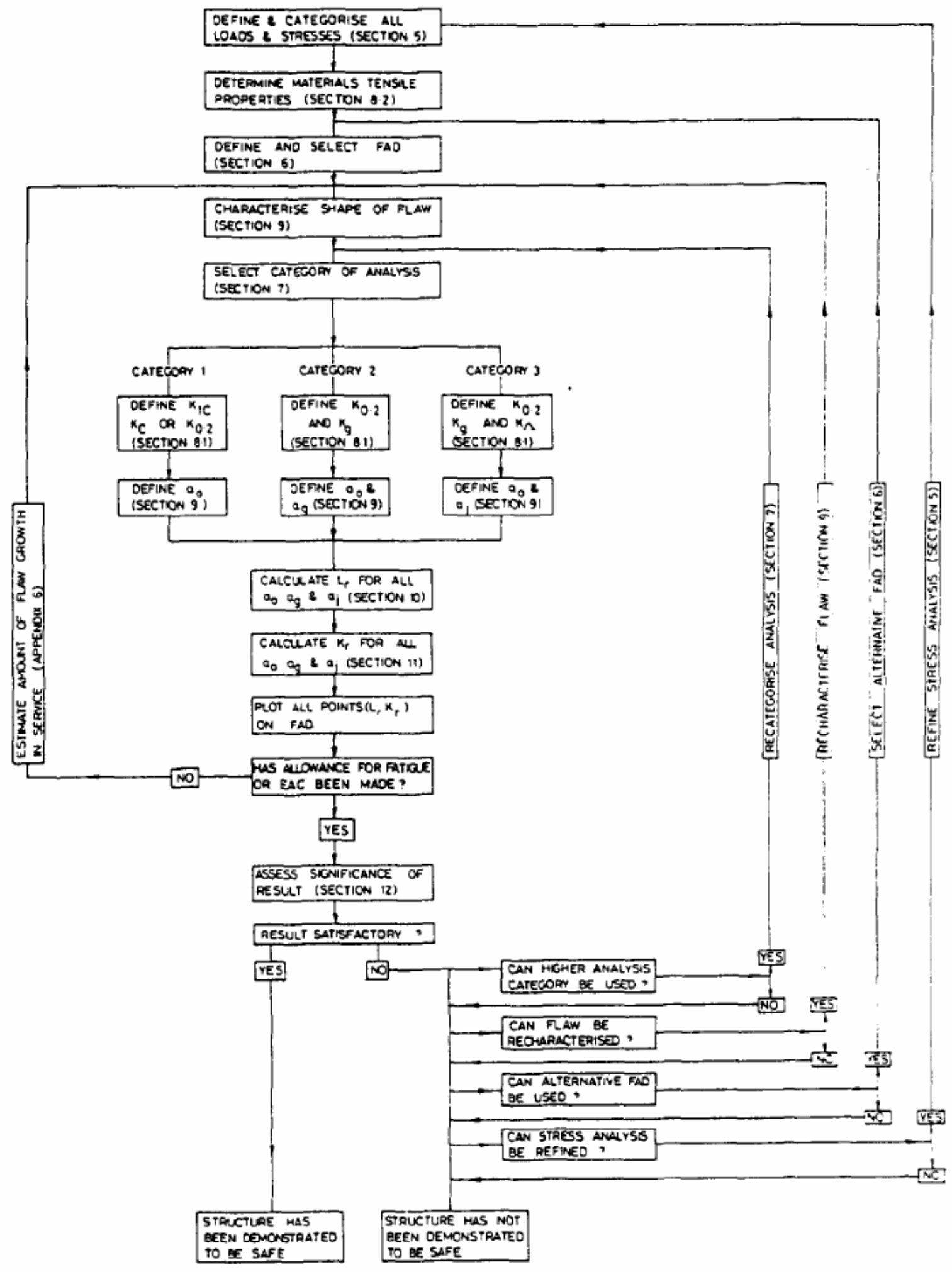

Figura 2.18. Diagrama de flujo para la aplicación del FAD [72]. 


\subsubsection{Breve descripción de los procedimientos}

Como se ha comentado anteriormente, la mayoría de los procedimientos de integridad estructural utilizan similares definiciones para los niveles de análisis planteados. En los siguientes párrafos se presenta un breve resumen de los mismos, remarcando las pequeñas diferencias entre ellos.

\section{Procedimiento R6}

El procedimiento R6 [73-75] ha sido continuamente desarrollado desde el año 1976. Nació en Gran Bretaña a través de la empresa Central Electricity Generating Borrad (CEGB) para dar solución a los requerimientos específicos de seguridad en las plantas nucleares. Después de la privatización de CEGB, el R6 pasó a ser responsabilidad de la empresa British Energy, la cuál continúa desarrollando este procedimiento en la actualidad, así como el R5 correspondiente a altas temperaturas.

Para la representación del FAD el método presenta tres opciones, las cuáles son análogas a las del procedimiento BS 7910, que será presentado en el apartado siguiente. La opción apropiada depende de los datos disponibles y de la precisión deseada.

- Opción 1. La línea de fallo se define a través de la envolvente inferior de las líneas de fallo de diferentes materiales metálicos, obtenidas a partir de la Opción 2. Esta opción es apropiada cuando no se dispone de los datos correspondientes a la curva de tracción, quedando definida por la expresión (2.14), sin embargo, su conservadurismo en determinados casos puede resultar excesivo.

- Opción 2. La línea de fallo se basa en la tensión de referencia del modelo, siendo necesario para su aplicación el conocimiento de la curva tensión-deformación del material en cuestión. Esta opción está definida por la expresión (2.15).

- Opción 3. Esta opción proporciona el análisis más preciso, pero es necesario el conocimiento de la integral $J$ aplicada en la estructura estudiada $\left(J_{a p p}\right)$, lo que conlleva la realización de una simulación numérica con la que poder evaluar dicho parámetro. La expresión (2.16), presentada al igual que las demás en los apartados anteriores, es la que define esta opción. 
Procedimiento BS 7910

Este procedimiento data del año 2000 [76] como sustitución al procedimiento BSI PD 6493 publicado por el British Standard Institute [77]. Se basa en el procedimiento R6, teniendo los siguientes niveles de evaluación:

- Nivel 1-Análisis preliminar. Utiliza un FAD muy sencillo, basado en diagramas conservadores, dicho FAD es un rectángulo definido por los ejes de coordenadas y las rectas $S_{r}^{\max }=0.8$ y $K_{r}^{\max }=0.7$, estando definidos $S_{r}$ y $K_{r}$ por las ecuaciones (2.18) y (2.10) respectivamente.

$$
\begin{gathered}
S_{r}=\frac{\sigma_{r e f}}{\sigma_{y}} \\
K_{r}=\frac{K_{I}}{K_{m a t}}
\end{gathered}
$$

- Nivel 2- Análisis normal. Presenta dos tipos, 2A y 2B, en función de los datos tensióndeformación que se conozcan. Para el nivel 2A basta con conocer los valores de $\sigma_{0.2} \mathrm{y}$ $\sigma_{u t s}$ para calcular $L_{r}^{\max }$ a partir de la expresión (2.13), ya que la línea de fallo se define según la ecuación (2.14). Para poder aplicar el nivel 2B es necesario conocer la curva completa tracción-deformación, pues la línea de fallo se define a través de la ecuación (2.15).

- Nivel 3-Análisis avanzado para desgarro dúctil. Este nivel es adecuado cuando se produce en la estructura el desgarro dúctil, al igual que el nivel anterior, presenta varios tipos de análisis, los cuales son el 3A, 3B y 3C. La aplicación de cada uno de estos tipos está supeditada a los datos que se posean del componente analizado, coincidiendo con las tres opciones de aplicación del procedimiento R6.

\section{Procedimiento SAQ}

En 1989, la empresa sueca SAQ Kontroll AB desarrolló este procedimiento en forma de manual y programa informático para su aplicación principalmente en la industria nuclear de 
su país, revisándose más tarde en 1996 [78,79]. Este procedimiento está basado en la Opción 1 de la Categoría 1 del procedimiento R6.

\section{Procedimiento EXXON}

En 1995 la empresa química EXXON publicó este procedimiento [80] basado principalmente en el antiguo procedimiento BSI PD 6493 y aplicable exclusivamente a componentes del sector químico.

\section{$\underline{\text { Procedimiento MPC }}$}

Este procedimiento [81] publicado en 1995 es similar al publicado por EXXON [80] y es aplicable a vasijas a presión, tanques y tuberías del sector químico. Para la representación del FAD el método propone dos líneas de fallo en función de los datos del componente que se conozcan, estas líneas coinciden con las propuestas en las opciones 1 y 3 del procedimiento R6, quedando definidas por las expresiones (2.14) y (2.16) respectivamente.

\section{Procedimiento API 579}

El procedimiento API 579 [82] ha sido desarrollado para proporcionar una herramienta de análisis de la integridad estructural de fisuras frecuentemente encontradas en vasijas de alta presión, tuberías y depósitos de almacenamiento. Existen tres niveles de análisis en función de las propiedades del material disponibles.

- Nivel 1. Es muy conservador, y está pensado para su utilización con muy poca información del componente y de los posibles defectos en el mismo.

- Nivel 2. Es más habitual, y utiliza la misma expresión general que los procedimientos R6 (Opción 1) y BS 7910 (Nivel 2A) para la definición de la línea de fallo. El diagrama FAD tiene una línea de corte en la zona de colapso definida mediante la tensión última a tracción $\sigma_{u t s}$ según la expresión (2.13).

- Nivel 3. Es el más avanzado y dispone de una gran flexibilidad para su aplicación en función de los datos disponibles. Las opciones que podemos encontrar dentro de este nivel de análisis son: 
- Método A. Nivel 2 con coeficientes de seguridad ajustados por el usuario o un análisis probabilístico.

- Método B. Determinación de un diagrama FAD específico para el material, similar a la Opción 2 del R6 y al Nivel 2A del BS 7910, es el más habitual.

- Método C. Diagrama FAD basado en la integral $J$, obtenido mediante un análisis de EF elasto-plástico, similar a la Opción 3 del procedimiento R6.

- Método D. Evaluación mediante las propiedades de desgarro dúctil.

- Método E. A partir del uso de otro procedimiento reconocido como el R6 o el BS 7910.

El apéndice $\mathrm{C}$ del procedimiento API 579 incluye una extensa librería de soluciones del factor de intensidad de tensiones y de la tensión de referencia aplicable a fisuras internas y externas en cilindros y otras geometrías comunes.

\subsubsection{Procedimientos basados en el uso de CDF}

Estos procedimientos utilizan el Diagrama de Fuerza Motriz de Agrietamiento (Crack Driving Force Diagram) como eje del análisis, se basan en el cálculo de la integral $J$ aplicada $\left(J_{a p p}\right)$, por lo que en ocasiones puede resultar compleja su aplicación. La Figura 2.19 muestra esquemáticamente la forma de utilizar este procedimiento. Básicamente hay que buscar la curva del diagrama CDF para una carga concreta que es tangente a la curva $J_{R}$. En este punto se alcanzaría la longitud de fisura crítica para la que se produce el fallo del componente.

Dentro de este grupo cabe destacar el procedimiento GE-EPRI, desarrollado en 1976 por Shih y Hutchinson [83], los cuáles propusieron una metodología avanzada para determinar propiedades de fractura en materiales que presentaban endurecimiento por deformación. Esta metodología fue desarrollada y validada por la General Electric Corporation durante los últimos años de los 70 y principios de los 80 , dando como resultado la publicación del método como manual ingenieril por el Electric Power Research Institute (EPRI) en 1881 [84]. 


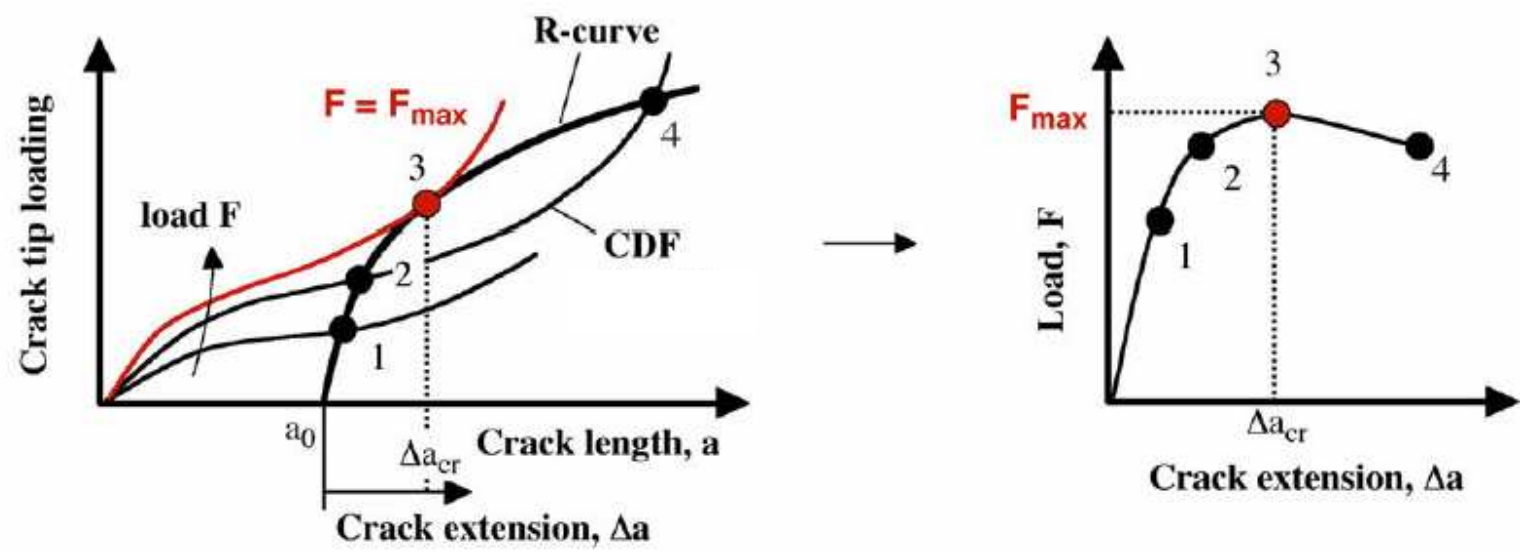

Figura 2.19. Esquema del procedimiento basado en el uso de CDF.

Este procedimiento es un medio válido para calcular la integral $J$ aplicada bajo condiciones elastoplásticas y completamente plásticas. Las componentes elástica y plástica de la integral $J$ se calculan por separado y se suman según la expresión (2.19) para dar el valor de $J$.

$$
J_{a p p}=J_{e}+J_{p}
$$

Dentro de este segundo grupo también se encuentra el procedimiento ETM, el cuál fue publicado por primera vez en 1994 [85] por el instituto alemán GKSS Research Center, sufriendo varias revisiones en los años posteriores [86-88]. El ETM usa manuales para determinar el valor del factor de intensidad de tensiones y extrapola su valor para el comportamiento plástico. La principal suposición que hace este método es que el comportamiento de la punta de fisura del componente se puede aproximar a partir de la curva ingenieril tensión-deformación obtenida en el ensayo de tracción uniaxial.

\subsubsection{Procedimientos basados en ambos conceptos}

Los siguientes procedimientos se pueden encuadrar en cualquiera de los dos apartados anteriores, dado que contemplan ambas aproximaciones (FAD y CDF) en el análisis desarrollado. Cabe destacar los procedimientos SINTAP y FITNET como los más aceptados internacionalmente. 
El SINTAP [89] nació en 1999 como consecuencia del proyecto "Structural Integrity Assessment Procedure for European Industry”, adscrito al programa de investigación BRITEEURAM, elaborado por un consorcio europeo de centros, instituciones y empresas. El listado de participantes se puede consultar en la página web del procedimiento [90] así como toda clase de información relacionada. El objetivo del SINTAP es ofrecer a la industria europea un procedimiento de evaluación con varios niveles de complejidad en función de las aplicaciones industriales y de los requerimientos de los usuarios. Integra las metodologías FAD y CDFD, es decir, aglutina las características de los procedimientos de evaluación existentes permitiendo al usuario la obtención de resultados convergentes en función del método utilizado.

La denominada red temática europea FITNET [91,92] (Fitness-for-service Network) comenzó sus trabajos en febrero de 2002 con los objetivos de establecer un procedimiento europeo y crear una norma europea para la evaluación de la integridad estructural. Esta red temática está parcialmente financiada por la Unión Europea y es coordinada por el GKSS en Alemania. Otras treinta empresas, centros de investigación y universidades de toda Europa proporcionan la información necesaria, en forma de "know-how" y de enlaces con otros programas de investigación. Las principales actividades que caracterizan el FITNET son las siguientes:

- La revisión de los procedimientos existentes y hallazgos de la investigación en lo que se refiere a la integridad estructural de los componentes metálicos.

- La generación de casos, ejemplos de estudio y otro material educativo.

- La celebración de seminarios y talleres de trabajo.

- El enlace con el Comité Europeo de Normalización (CEN).

Otros procedimientos que podemos encontrar dentro de este tercer grupo son: el ASME XI [93], el EN J [94] y el DPFAD [95,97].

El procedimiento ASME XI [93], contiene normas para la realización de inspecciones rutinarias, la reparación y el reemplazamiento de componentes en servicio de plantas nucleares. Se requiere un programa obligatorio de inspecciones regulares y pruebas de seguridad. Se incluyen también métodos de caracterización de tamaño de grieta e 
inspecciones en servicio para determinar el estado de los componentes por medio de técnicas no destructivas.

El procedimiento EN J [94], aborda de una forma ingenieril la evaluación de la integridad estructural. El método se basa en el cálculo del factor de intensidad de tensiones equivalente en el momento en que se alcanza el límite elástico en el componente, aplicando posteriormente una serie de correcciones de cálculo.

Y finalmente, el procedimiento DPFAD, desarrollado por Bloom y Malik [95,96] bajo el nombre "Deformation Plasticity Failure Assessment Diagram", se basa en la evaluación de varios puntos en un FAD obtenido a partir de las consideraciones para el cálculo de la integral $J$ del procedimiento GE-EPRI.

\subsection{CONSIDERACIONES FINALES}

Como se ha podido comprobar en este capítulo han sido muchos los autores que han utilizado el SPT como eje principal de sus investigaciones, cubriendo un amplio abanico en lo que a campos de aplicación se refiere, desde su utilización, por ejemplo, en el campo nuclear hasta su uso en el campo de la medicina. De entre todos estos trabajos llevados a cabo durante estas últimas décadas uno de los más relevantes, para el desarrollo de la presente Tesis, es el realizado por Mao y Takahashi [4], debido a que representa una base sólida en la que fundamentar el procedimiento para la estimación de los parámetros elastoplásticos del material utilizado, recogido en el apartado 4.4 de la presente Tesis. También cabe destacar que han sido muy pocos los autores que durante este tiempo han utilizado en sus investigaciones el SPT con probetas previamente fisuradas, no habiendo ninguno de ellos hecho uso de la forma de la prefisura empleada en este trabajo, la cuál se expone en el capítulo siguiente.

En cuanto a los procedimientos de integridad estructural recogidos en el apartado 2.4, los cuáles son capaces de determinar si el fallo del componente se produce por colapso plástico, por fractura frágil o por una mezcla de las dos anteriores, se puede decir que muchos de ellos han sido desarrollados por empresas para su aplicación en su propio nicho de mercado. Como se ha visto, dentro de la Unión Europea se está desarrollando en la actualidad un ambicioso 
proyecto denominado FITNET, con el objetivo de establecer un procedimiento europeo y crear una norma europea para la evaluación de la integridad estructural, y de esta forma unificar criterios en este campo de aplicación.

Los procedimientos basados en el uso del diagrama FAD más importantes y muy similares entre sí, son el R6, BS 7910 y el API 579. De forma general y sin ceñirse a un procedimiento en concreto, uno de los pilares fundamentales de la metodología desarrollada en la presente Tesis es el uso del diagrama FAD, el cuál presenta varias opciones para la representación de la curva que lo define en función de los datos disponibles del material utilizado y del grado de precisión que se desee.

Dentro de este mismo apartado han quedado reflejadas claramente las consideraciones a tener en cuenta en la aplicación de cada una de las opciones de FAD. En este trabajo se ha hecho uso de las tres opciones de FAD disponibles con el objetivo de establecer el grado de precisión que se alcanza con cada una de ellas aplicando la metodología desarrollada. Teóricamente, la Opción 1 es la que presenta un grado de exactitud menor y una mayor facilidad de aplicación, mientras que con la Opción 3 sucede todo lo contrario, proporciona un grado de exactitud mayor a consta de una mayor complejidad en su aplicación. 



\section{CAPÍTULO 3}

\section{METODOLOGÍA}

\subsection{INTRODUCCIÓN}

La metodología que se presenta en este capítulo hace uso del diagrama FAD de una forma poco convencional, encaminado, como se verá posteriormente, a la determinación de la tenacidad del material, $K_{m a t}$. La línea de fallo, resultante de la aplicación de los procedimientos de integridad estructural, es la encargada de predecir el instante en el que se inicia la propagación de una fisura en un componente fisurado. En este sentido, el ensayo SPT convencional, donde no existe una fisura inicial, no parece adecuado para la determinación directa de los parámetros de fractura convencionales ( $K$ ó $J$ ). Puede ser utilizado, eso sí, para determinar parámetros de daño, que inician y desarrollan la rotura final de la pieza. En este trabajo, se pretende estimar directamente los parámetros de fractura ( $K$ ó $J$ ) mediante el ensayo SPT, lo cuál requiere la consideración de una probeta fisurada. Han sido varias las geometrías analizadas en el entorno de trabajo en el que se ha desarrollado la presente Tesis. Las opciones que se han barajado aparecen reflejadas en la Figura 3.1. Se han considerado fisuras pasantes en todo el espesor, de diferentes longitudes, desde antes de la línea de carga (a) hasta después de la línea de carga (b). Evidentemente, la elección de un tipo u otro de fisura debe obedecer a criterios científicos, pero también deben considerarse criterios de viabilidad. La fisura tipo (d), central no pasante, en unas dimensiones tan reducidas de probeta resulta a priori inviable de mecanizar. Las fisuras tipo (a) y (b) no han resultado muy prácticas desde el punto de vista de longitud de propagación, y en cualquier caso el espesor de la 
probeta haría que el valor de $K$ fuese muy cercano a condiciones de tensión plana. La fisura tipo (c), longitudinal no pasante, parece en primera instancia una de las opciones factibles, aunque evidentemente también presenta sus limitaciones (reducido espesor e inicio de propagación de fisura en la zona cercana al punzón). Sin embargo, se cree que puede utilizarse para estimar las condiciones de inicio, con las particularidades que los resultados obtenidos puedan presentar.
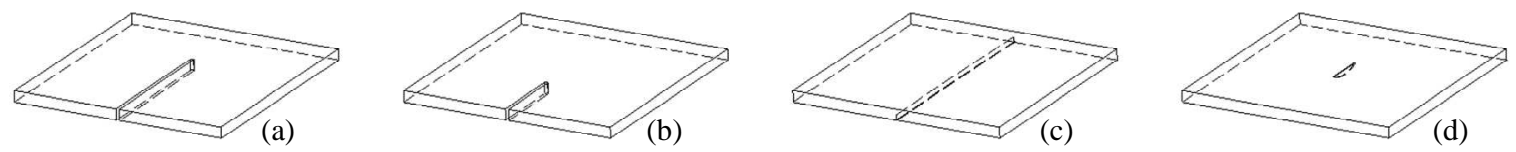

Figura 3.1. Tipos de fisura inicial en probetas SPT.

\subsection{DEFINICIÓN DE LAS PROBETAS}

En el desarrollo de la presente Tesis se han realizado una serie de ensayos SPT con diferentes tipos de probeta, pudiendo englobarlas en dos grandes grupos, las probetas SPT convencionales ( $\sin$ fisura inicial), de dimensiones $10 \times 10 \times 0.5 \mathrm{~mm}$ y $20 \times 20 \mathrm{x} 1 \mathrm{~mm}$, y las probetas SPT prefisuradas, de dimensiones 20x20x1 $\mathrm{mm}$, con una fisura inicial de tipo longitudinal no pasante. Como se verá más adelante, las primeras han sido utilizadas para establecer un procedimiento con el que poder estimar las propiedades elastoplásticas del material, mientras que las segundas han sido las elegidas para llevar a cabo una serie de ensayos SPT imprescindibles en la aplicación de la metodología presentada.

A simple vista se observa que el grupo de probetas destinadas para la realización de SPT prefisurados están escaladas al doble del tamaño de las probetas SPT convencionales. Este escalado se ha efectuado para poder trabajar de manera factible con diferentes longitudes de prefisuración, a fin de estimar la profundidad óptima para la consecución de los objetivos planteados. Dicho escalado también afecta al utillaje del ensayo, como se puede comprobar en la Tabla 3.1 que recoge los valores principales del utillaje para ambos tamaños de probeta.

La generación de la fisura inicial en las probetas SPT, previa al ensayo, se ha realizado a través de microfisuración por láser. Tras una calibración inicial, el láser, en forma de haz de 
pulsos de diámetro $30 \mu \mathrm{m}$, es aplicado longitudinalmente a partir de la cara inferior, desde el centro de un lado de la probeta hacia el centro del lado opuesto, como se puede observar en la Figura 3.2. En dicha figura se muestra el fondo de entalla conseguido con esta técnica. Cabe destacar que la profundidad obtenida presenta cierta irregularidad como se verá en capítulos posteriores. Para los ensayos SPT con probetas prefisuradas se han utilizado profundidades de fisura teórica de 0.2, 0.3, 0.4 y 0.5 mm, obteniendo así una relación $a / t$ teórica de 0.2, 0.3, 0.4 y 0.5 respectivamente. Los valores de estas profundidades teóricas de fisura, así como la calidad de las fisuras obtenidas, se han comprobado posteriormente a la realización de los ensayos, mediante técnicas de microscopía óptica de barrido (SEM).

Tabla 3.1. Medidas de las probetas y del utillaje utilizado en los ensayos SPT.

\begin{tabular}{|l|c|c|}
\cline { 2 - 3 } \multicolumn{1}{c|}{} & $\begin{array}{c}\text { Ensayos SPT } \\
\text { prefisurados }\end{array}$ & $\begin{array}{c}\text { Ensayos } S P T \\
\text { típicos }\end{array}$ \\
\hline Dimensiones probeta, $b \times b \times t(m m)$ & $20 \times 20 \times 1$ & $10 \times 10 \times 0.5$ \\
\hline Diámetro del punzón, $d(\mathrm{~mm})$ & 5 & 2.5 \\
\hline Diámetro matriz inferior, $d_{m}(\mathrm{~mm})$ & 8 & 4 \\
\hline Radio de acuerdo matriz inf., $r(\mathrm{~mm})$ & 1 & 0.5 \\
\hline
\end{tabular}
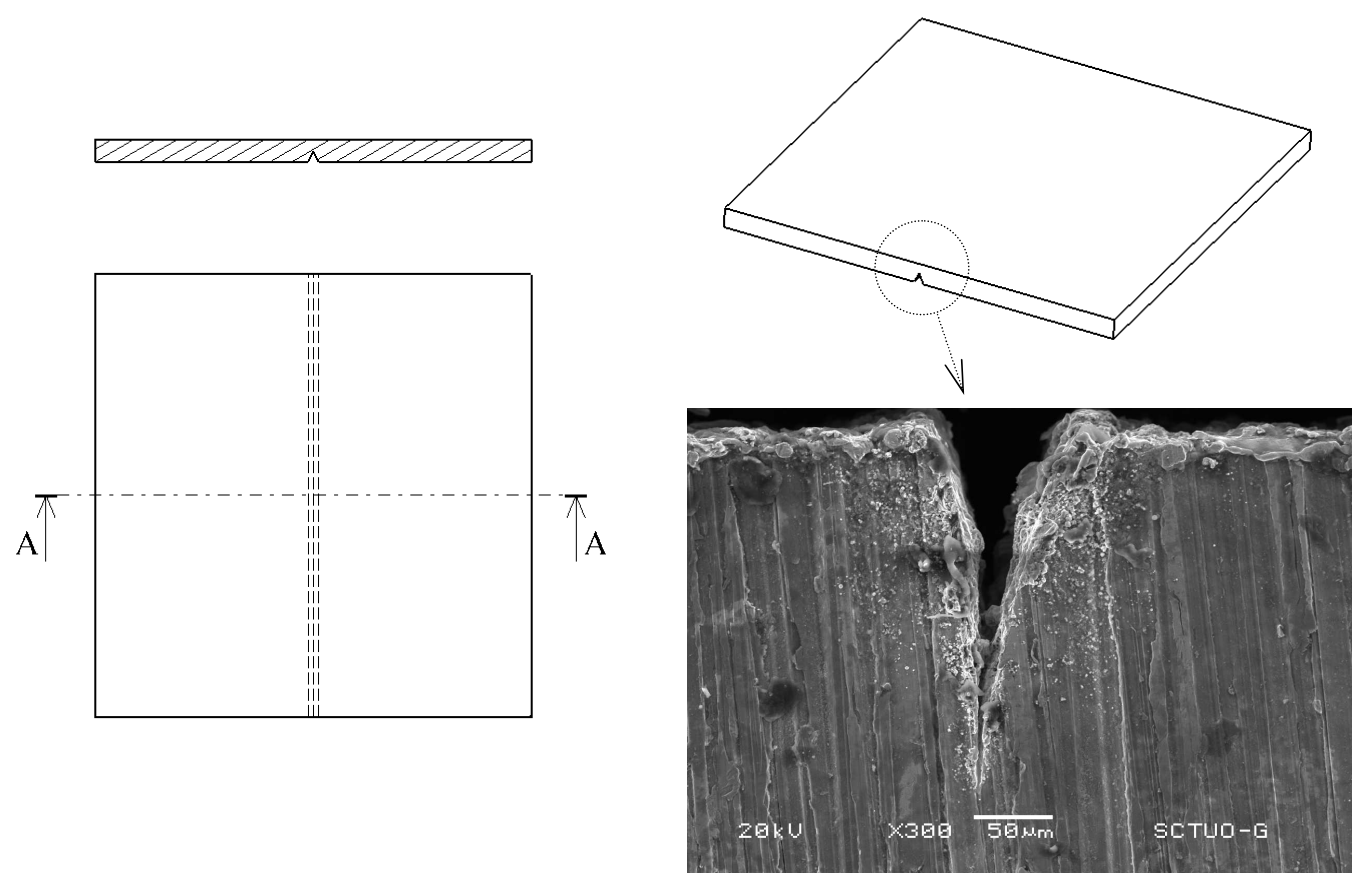

Figura 3.2. Probeta SPT prefisurada. 


\subsection{ESQUEMA METODOLÓGICO}

El diagrama de fallo es el diagrama que define cuando el componente fisurado alcanza las condiciones de rotura. Es un gráfico de doble entrada, donde en el eje horizontal está representando el grado de plastificación alcanzado por la probeta en el instante de fallo, a través de un parámetro denominado ratio de carga $\left(L_{r}\right)$, que viene definido por la expresión (2.11), que recordando era $L_{r}=\sigma_{r e f} / \sigma_{y}=P_{a p l} / P_{U}$. El eje vertical representa el ratio del factor de intensidad de tensiones alcanzado en el instante de fallo, normalizado con respecto a la tenacidad del material, quedando representado a través del ratio de tenacidad $\left(K_{r}\right)$, que dependiendo de la categoría de análisis elegida queda definido por las diferentes ecuaciones proporcionadas por la expresión (2.17). El esquema de la metodología presentada en esta Tesis se puede observar en la Figura 3.3. Como se puede comprobar dicha metodología gira entorno al uso del diagrama de fallo, donde la entrada vertical $K_{r}$ es desconocida, al serlo $K_{m a t}$, por lo que se comienza entrando en el diagrama FAD por el eje horizontal a partir del valor de $L_{r}=P_{a p l} / P_{U}$. Para evaluar $L_{r}$ es necesario calcular $P_{a p l}$ y $P_{U}$. El primer paso a realizar es la determinación mediante simulación numérica, de la carga de colapso plástico de la probeta fisurada $P_{U}$. Paralelamente se realizarán una serie de ensayos SPT sobre probetas prefisuradas para establecer la carga aplicada sobre la probeta en el punto de inicio de la propagación de la fisura $P_{a p l}$. Diferentes métodos serán presentados para alcanzar este objetivo.

Con estos dos valores obtenidos se calcula el valor de $L_{r}=P_{a p l} / P_{U}$. Una vez establecido este valor, es decir, conocido el valor de abscisas en el FAD, obtenemos el punto de corte de dicha abscisa con la curva del diagrama, la cuál puede ser obtenida con cualquiera de las tres opciones disponibles para su representación. Este punto define el instante en el que comienza a propagar la fisura en la probeta analizada, quedando definido el valor de la ordenada de dicho punto, la cuál corresponde al valor de $K_{r}=K_{I} / K_{m a t}$. Por otro lado, a partir de la simulación numérica de la probeta prefisurada se calcula el factor de intensidad de tensiones $K_{I}$ correspondiente a $P_{a p l}$ en el punto de inicio de propagación de fisura. Con los valores de $K_{r}$ y $K_{I}\left(P_{a p l}\right)$ determinados, el último paso de esta metodología es estimar el valor de $K_{m a t}$. 


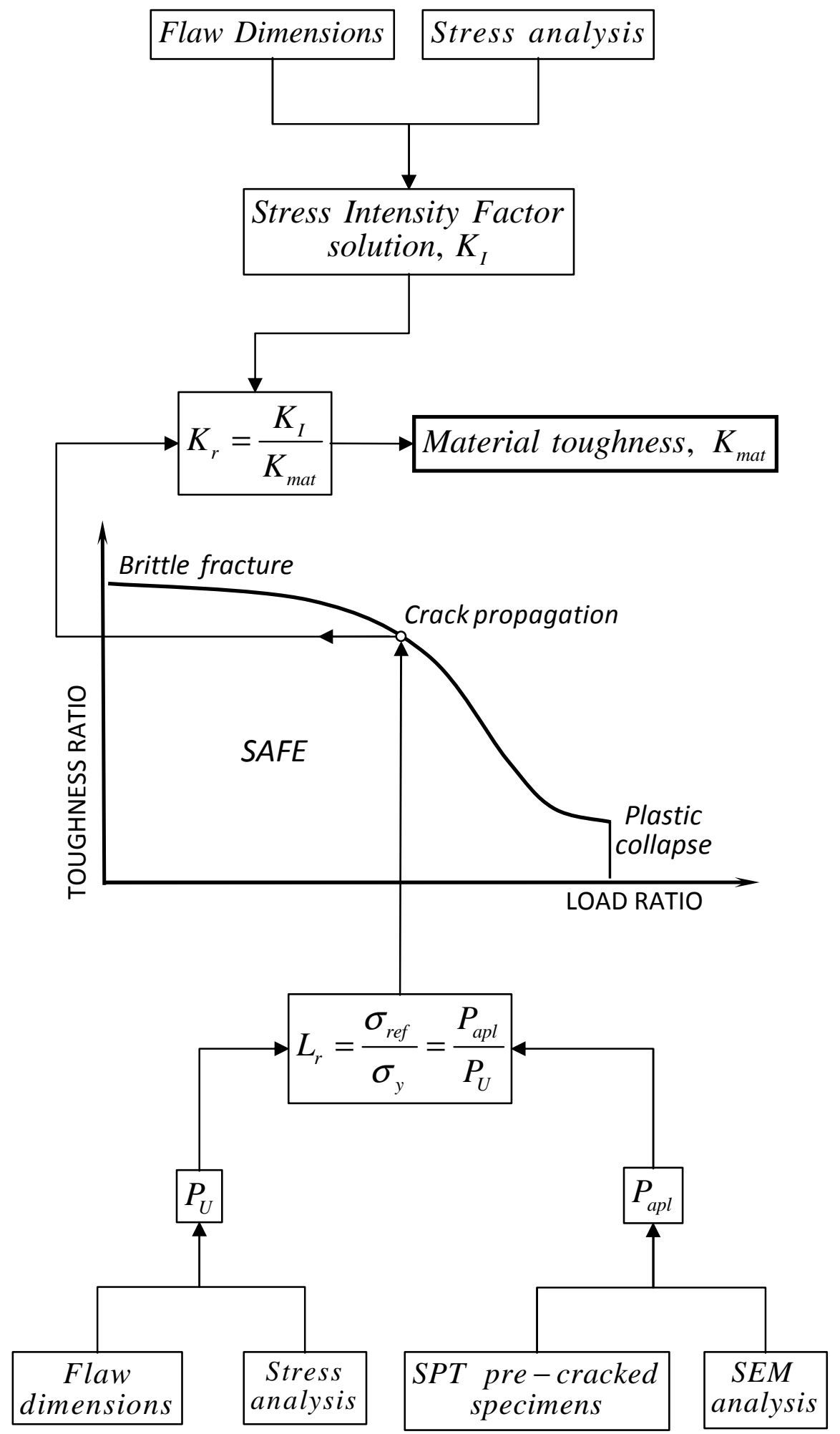

Figura 3.3. Esquema de la metodología desarrollada para el cálculo de $K_{\text {mat }}$. 


\subsection{DETERMINACIÓN DEL RATIO DE CARGA $L_{r}$}

El nivel de plastificación de la probeta analizada es tenido en cuenta a través del ratio de carga $L_{r}$. Como se ha comentado anteriormente, para determinar el valor de $L_{r}$ es necesario calcular el valor de la carga aplicada en el momento de inicio del fallo $P_{a p l}$, y de la carga de colapso plástico $P_{U}$. Para ello es necesario seguir los pasos que se explican en detalle en los siguientes apartados.

\subsubsection{Determinación de la carga aplicada en el inicio de propagación de fisura, $\boldsymbol{P}_{\text {apl }}$}

La Figura 3.4 presenta las curvas carga-desplazamiento obtenidas en el ensayo SPT con probetas prefisuradas de dimensiones $20 \times 20 \times 1 \mathrm{~mm}$, para el material analizado en la presente Tesis, en función de si la relación $a / t$ es mayor o menor de 0.5. Analizando la forma que presentan dichas curvas, es posible distinguir diferentes regiones en cada una de ellas. Para una mayor comprensión de estas curvas, también se han representado las obtenidas en un ensayo SPT sin fisura y en la simulación de una fisura completamente pasante, es decir, como si estuviese formada por dos mitades unidas sólo por las fuerzas de contacto (probeta rota en dos mitades). Este último caso representa la situación en la que se encuentra la probeta completamente rota.

Para la curva correspondiente a una relación $a / t>0.5$, la región I correspondería al comportamiento puramente elástico de la probeta. En esta región tanto la probeta como el utillaje se reposicionan ligeramente, se produce el fenómeno de indentación, e incluso la rotura de pequeños ligamentos dejados por el láser durante el mecanizado de la fisura. La región II correspondería con la transición elastoplástica, ya que en algunas zonas de la probeta comienza a predominar la deformación plástica, especialmente la zona en contacto con el punzón. En la tercera región tiene lugar la progresiva abertura de los labios de la fisura y la plastificación creciente de la probeta, y como consecuencia final de esto, el comienzo y propagación de la fisura, hasta llegar al quiebro que se produce en la curva, entre la región III y IV, en el cuál la fisura se hace pasante a lo largo del espesor de la probeta. A partir del inicio de la región IV se produce rápidamente el desgarro de la probeta hasta alcanzar los bordes de la matriz, instante en el que la probeta tiende a comportarse como dos mitades 
desacopladas (fisura pasante). La quinta y última región se puede decir que comienza cuando la curva carga-desplazamiento se ajusta al comportamiento de dos mitades, como se puede ver en la Figura 3.4.

En la curva correspondiente a una relación $a / t<0.5$ se puede llegar a distinguir de nuevo hasta cinco regiones más o menos diferenciadas. Las tres primeras se corresponderían con las de la curva anterior, con la salvedad de que la región III es mucho mayor en este caso, alcanzando la probeta una mayor capacidad de carga al ser mayor el ligamento resistente. A lo largo de la región III se inicia la fisura y crece hasta hacerse pasante, cuyo valor en este caso coincide con el máximo de la curva carga-desplazamiento. En ese instante se produce un descenso brusco de la capacidad resistente de la probeta. La región IV comprende dicho decremento, en ella la fisura propaga en ambos sentidos por todo el espesor, hasta llegar a la zona de empotramiento, momento en el que la probeta, al igual que antes, se comporta como dos mitades desacopladas. La última región correspondería al traspaso total del punzón, a través de las dos mitades desacopladas.

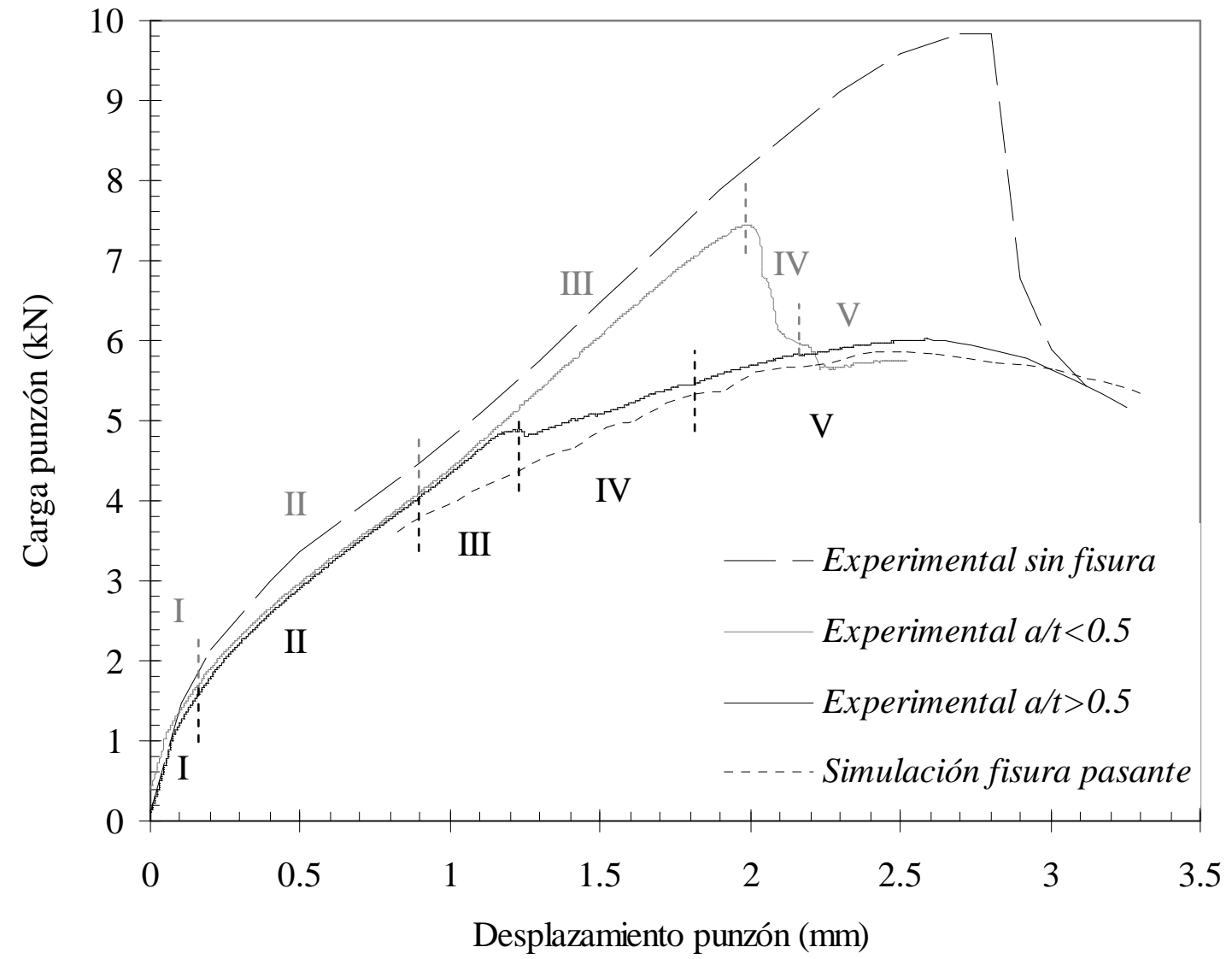

Figura 3.4. Curvas carga-desplazamiento del ensayo SPT sobre probeta prefisurada. 
Como se ha podido intuir, la diferencia existente entre las dos curvas radica principalmente en la capacidad de carga que puede soportar la probeta hasta el instante en el que la fisura se inicia, haciéndose pasante a lo largo del espesor, y propagando rápidamente hasta la zona del empotramiento. Una vez alcanzado este instante el comportamiento de la probeta se intenta estabilizar de una forma u otra, asemejándose al exhibido por dos mitades desacopladas, obteniendo de esta manera las curvas anteriores en función de la relación $a / t$.

Como se ha podido comprobar, el instante de inicio de la propagación no queda claramente manifestado en la curva carga-desplazamiento de la probeta SPT prefisurada. En cambio, el instante en el que la fisura se hace pasante en todo el espesor si que está identificado en ambas curvas, por el quiebro de la curva en la primera y por el descenso brusco de la capacidad resistente en la segunda. En consecuencia, para determinar el valor de la carga aplicada sobre la probeta en el instante de inicio de la propagación de fisura se han desarrollado tres posibles métodos, que se explican en detalle en los siguientes apartados. Existen otras técnicas que podrían considerarse en un futuro para la identificación del instante de inicio de propagación, como la emisión acústica.

\subsubsection{Ensayos interrumpidos}

Este método consiste en realizar al menos una batería de ensayos interrumpidos sobre probetas SPT prefisuradas del material estudiado, en el entorno del punto donde se prevé la iniciación. Dichos ensayos se ejecutarían con una velocidad de desplazamiento del punzón relativamente baja de $\mathrm{v}=0.8 \mathrm{~mm} / \mathrm{min}$, con el objetivo de que los ensayos sean considerados como cuasiestáticos. Una vez realizados los ensayos interrumpidos se efectúa un primer análisis visual y un posterior análisis SEM de las probetas para determinar si la fisura se ha iniciado o no en el punto en el que se ha detenido el ensayo. La rotura de la probeta de forma frágil, mediante nitrógeno líquido, es necesaria para favorecer el análisis fractográfico. El inconveniente que presenta este método es la laboriosidad del ensayo y la complejidad de acertar con el punto de iniciación.

En el supuesto de realizar varias baterías de ensayos SPT interrumpidos con diferentes valores de la profundidad de fisura inicial $a$, a cada una de ellas le corresponderá un valor de $P_{a p l}$ diferente. En general se ha observado que el inicio de la fisuración está entorno al 85\% de la carga que produce la fisura pasante. 
Para no tener que realizar un elevado número de ensayos se puede efectuar un primer ensayo completo, identificar el instante en el que la fisura se hace pasante, es decir, el quiebro de la curva si la relación $a / t>0.5$ o el descenso brusco de la capacidad resistente si la relación $a / t<0.5$ y realizar ensayos interrumpidos en el entorno del $85 \%$ de la carga correspondiente a la fisura pasante.

Este método se puede utilizar para cualquier relación $a / t$, al igual que el método de la flexibilidad, mientras que el método de evaluación de la pendiente es preferible emplearlo cuando $a / t<0.5$, ya que la región III abarca un tramo mayor de la curva cargadesplazamiento, favoreciendo así su aplicación.

\subsubsection{Método de evaluación de la pendiente de la curva}

Este método consiste en analizar la variación de la pendiente en la curva cargadesplazamiento a lo largo de la región III. Al igual que sucede en la curva correspondiente a las probetas convencionales de SPT, el comportamiento observado en esta región marca un carácter bastante lineal y en consecuencia es posible ajustarlo mediante una recta (pendiente constante), a excepción del último tramo a partir del inicio de propagación de fisura, en donde se produce el decremento de la pendiente en la curva conforme la fisura va creciendo hasta hacerse pasante a lo largo del espesor. Este descenso en la pendiente puede estar también condicionado por la plastificación elevada de la probeta.

La pendiente en un punto se calcula como el incremento de carga frente al incremento de desplazamiento con respecto al punto anterior. Para evitar los problemas de ruido, típicos de la adquisición de datos, y favorecer la visualización de la variación de la pendiente, se ha utilizado como valor de la pendiente en un punto la media de los valores de los cinco puntos anteriores y posteriores, además del suyo propio.

Representando este valor frente al desplazamiento del punzón se obtiene la curva típica mostrada en la Figura 3.5, en la que se puede identificar el valor del desplazamiento del punzón donde la pendiente de la curva deja de ser constante. Este punto correspondería con el inicio de propagación de fisura, por lo que quedaría determinado el valor de carga que se alcanza en este punto, o lo que es lo mismo, el valor de $P_{a p l}$. De esta forma se podría continuar con la aplicación de la metodología desarrollada. 

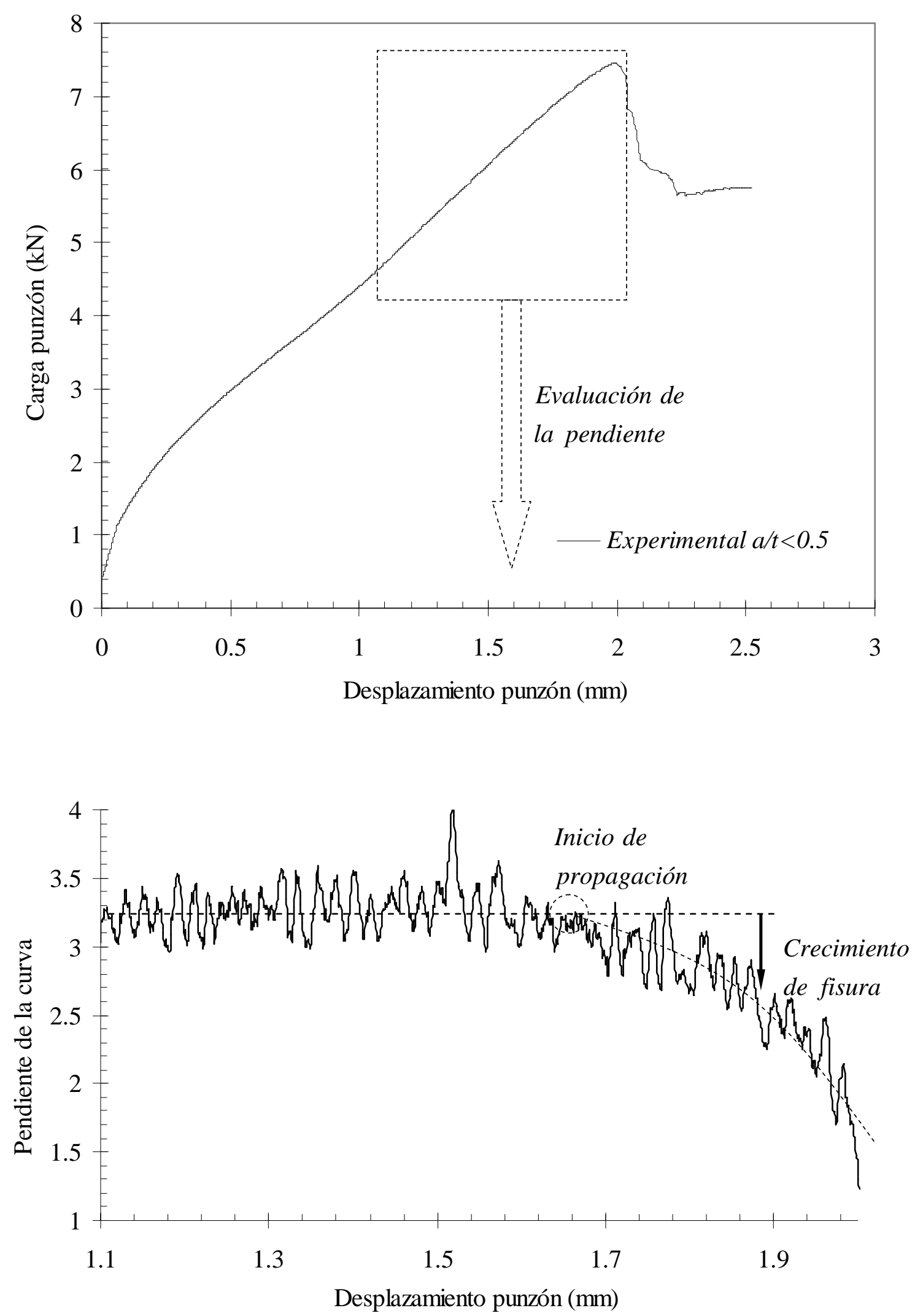

Figura 3.5. Método de evaluación de la pendiente de la curva. 


\subsubsection{Método de la flexibilidad o de las descargas elásticas}

Este método es similar al utilizado con probetas CT, y es considerado en principio el más fiable dado que al basarse en las descargas elásticas se evita el efecto de la plastificación en la detección del inicio de propagación de fisura. Consiste en la realización de un ensayo SPT prefisurado en el que se llevan a cabo una serie de cargas y descargas, para la posterior evaluación de la variación de la pendiente de cada una de las descargas realizadas. La curva característica del ensayo se muestra en la Figura 3.6, donde también se puede ver la variación de la pendiente de las diferentes cargas realizadas. Esta variación es linealmente creciente, dado que la geometría de la probeta varía continuamente debido al alto grado de plastificación, a diferencia de lo que ocurre en la probeta CT donde la variación es constante (la geometría no varía) hasta el instante en el que se produce el inicio de propagación de fisura, donde se observa una pérdida progresiva de rigidez de la probeta. En nuestro caso, el inicio de la propagación está relacionado con la pérdida de linealidad de la variación de la pendiente de las descargas, como se puede ver en la Figura 3.6, por lo que de nuevo quedaría determinado el valor de carga $P_{a p l}$ que se alcanza en este punto.

Obsérvese el hecho importante de que la pendiente de las descargas elásticas es independiente de la forma de la curva carga-desplazamiento hasta el momento de iniciación. Es decir, no se ve influida por el tramo I, II o III de la curva carga-desplazamiento. Únicamente se detecta la variación en el instante de inicio de la fisuración. El método de obtener la pendiente de las descargas es importante dado que debe efectuarse de la manera más exacta posible. En nuestro caso, dicha pendiente se ha calculado ajustando los datos de cada una de las descargas (d.e.) a una recta definida por la expresión (3.1),

$P=m_{\text {d.e. }} \cdot \Delta p+n_{0 \text { d.e. }}$

donde $P$ y $\Delta p$ son la carga y el desplazamiento del punzón, y $m_{\text {d.e. }}$ y $n_{0 \text { d.e. }}$ son la pendiente $\mathrm{y}$ la ordenada en el origen de la recta respectivamente. Por lo tanto, el valor de $m_{\text {d.e. }}$ es el que se toma como valor de la pendiente en cada una de las descargas realizadas en el ensayo. Representando los diferentes valores de $m_{\text {d.e. }}$ frente al desplazamiento del punzón, es posible identificar la pérdida de linealidad comentada anteriormente, con lo que el inicio de propagación quedaría determinado (Figura 3.7). 

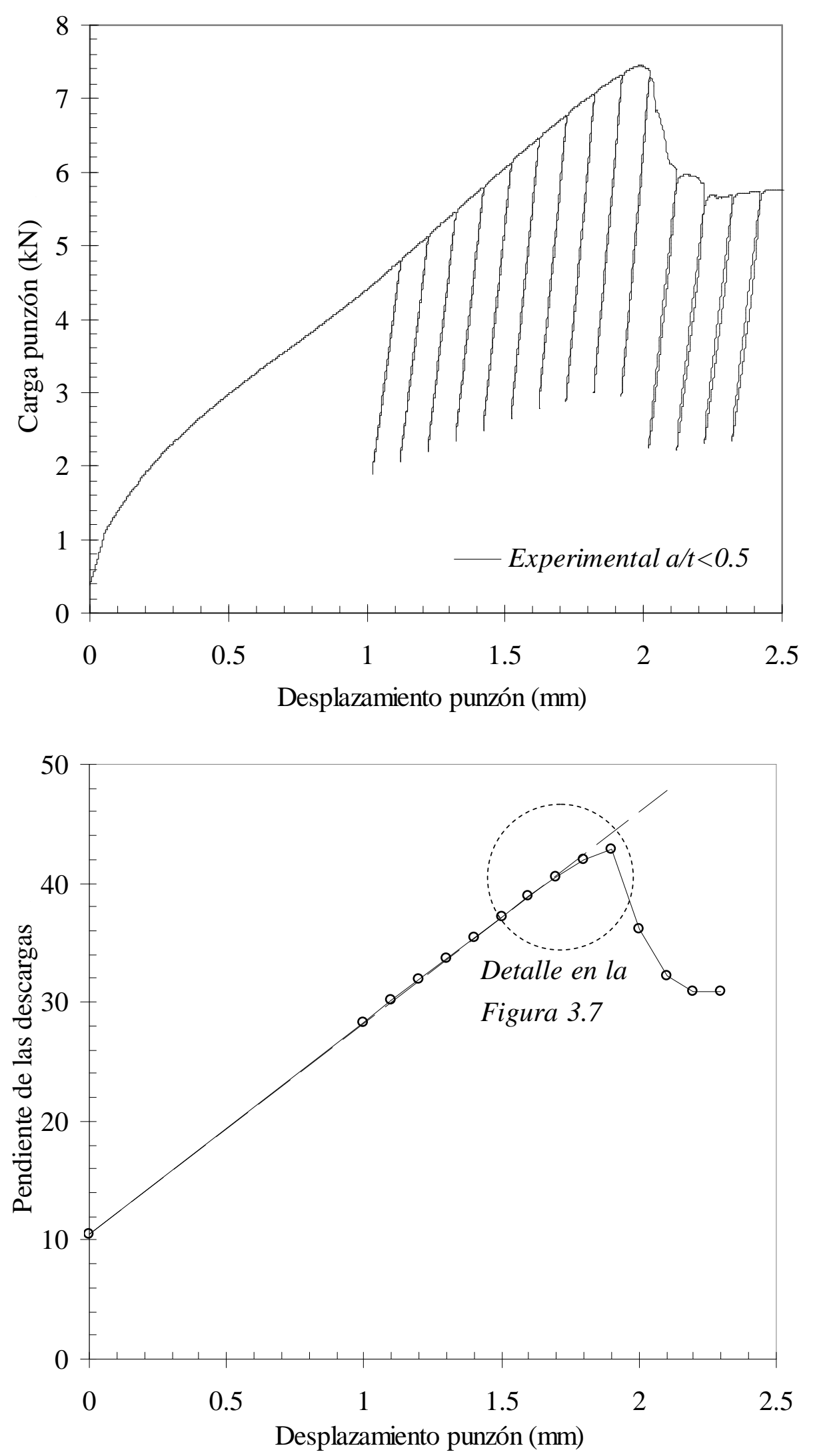

Figura 3.6. Método de la flexibilidad. 


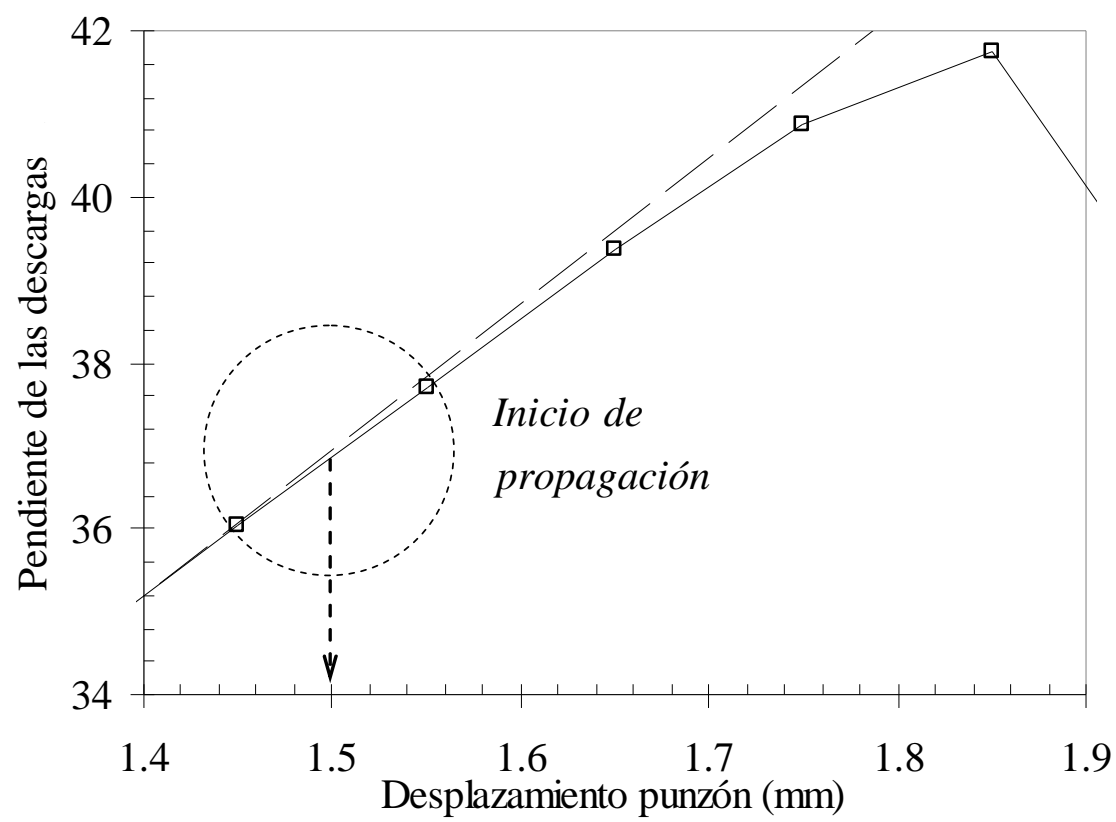

Figura 3.7. Detalle de la curva pendiente-desplazamiento de la Figura 3.6.

Finalmente, para estimar el ratio de carga $L_{r}$, nos quedaría únicamente por determinar el valor de la carga de colapso plástico $P_{U}$, para cada una de las probetas SPT prefisuradas utilizadas.

\subsubsection{Determinación de la carga de colapso plástico, $\boldsymbol{P}_{U}$}

Para determinar la carga de colapso plástico de la probeta prefisurada $P_{U}$, es necesario efectuar un análisis por elementos finitos elastoplástico de la geometría fisurada. Existen dos definiciones posibles de la carga de colapso plástico: el colapso global y el colapso de la sección neta. En la presente Tesis se ha utilizado el segundo de los procedimientos, por considerarse más preciso el resultado obtenido [82].

Para los dos tamaños de probeta analizados se ha obtenido una superficie de respuesta, que contempla el efecto de las propiedades elastoplásticas del material y de la geometría de la fisura en el valor de la carga de colapso plástico. De este modo la metodología presentada puede aplicarse sin necesidad de tener que realizar el complejo cálculo numérico de $P_{U}$. La 
obtención de la superficie de respuesta de la carga de colapso plástico $P_{U}$, ha sido realizada para el caso de un material cuya ley de comportamiento se ajuste a una ecuación tipo Ramberg Osgood [97] definida por la expresión (3.2).

$\varepsilon=\varepsilon_{e}+\varepsilon_{p}=\frac{\sigma}{E}+K_{1} \cdot\left(\frac{\sigma}{E}\right)^{n}$

Donde $K_{1}$ y $n$ son parámetros que describen el comportamiento plástico del material. Introduciendo el límite elástico $\sigma_{y}$ y un nuevo parámetro $\alpha=K_{1} \cdot\left(\sigma_{y} / E\right)^{n-1}$, la parte plástica de la ecuación (3.2) se puede sustituir por la expresión (3.3), quedando redefinida la ecuación de Ramberg Osgood según la expresión (3.4).

$$
\begin{aligned}
& K_{1} \cdot\left(\frac{\sigma}{E}\right)^{n}=\frac{\alpha \cdot \sigma_{y}}{E} \cdot\left(\frac{\sigma}{\sigma_{y}}\right)^{n} \\
& \varepsilon=\frac{\sigma}{E}+\frac{\alpha \cdot \sigma_{y}}{E} \cdot\left(\frac{\sigma}{\sigma_{y}}\right)^{n}
\end{aligned}
$$

La utilización del modelo de Ramberg Osgood implica que la deformación plástica está presente incluso para pequeños niveles de tensión, aunque es prácticamente despreciable comparada con la deformación elástica. Por otro lado, para niveles de tensión superiores a $\sigma_{y}$, la deformación plástica comienza progresivamente a ser mayor que la deformación elástica. Cuando $\sigma=\sigma_{y}$ según la expresión (3.4) se obtiene que $\varepsilon=(1+\alpha) \cdot \sigma_{y} / E$, obteniendo las componentes elástica y plástica de la deformación tal y como se puede observar en la Figura 3.8.

Comúnmente en los aceros se acepta que la deformación plástica comienza a tenerse en cuenta cuando esta alcanza un valor del $0.2 \%$, es decir, cuando el término $\alpha \cdot \sigma_{y} / E=0.002$, con lo que el modelo de Ramberg Osgood definido en (3.4) puede ser simplificado según la expresión (3.5), en la que $\sigma_{y}=\sigma_{0.2}$.

$$
\varepsilon=\frac{\sigma}{E}+0.002 \cdot\left(\frac{\sigma}{\sigma_{0.2}}\right)^{n}
$$


Donde $\sigma_{0.2}$ es el límite elástico para una deformación plástica del $0.2 \%$, y $n$ es el exponente de endurecimiento que suele variar entre 4 y 50 para la mayoría de los aceros [97]. De esta manera queda definido el comportamiento del material a través de los parámetros $E, \sigma_{0.2} \mathrm{y}$ $n$. Estos parámetros son fundamentales, a la hora de establecer los valores de la carga de colapso plástico $P_{U}$, de las probetas prefisuradas.

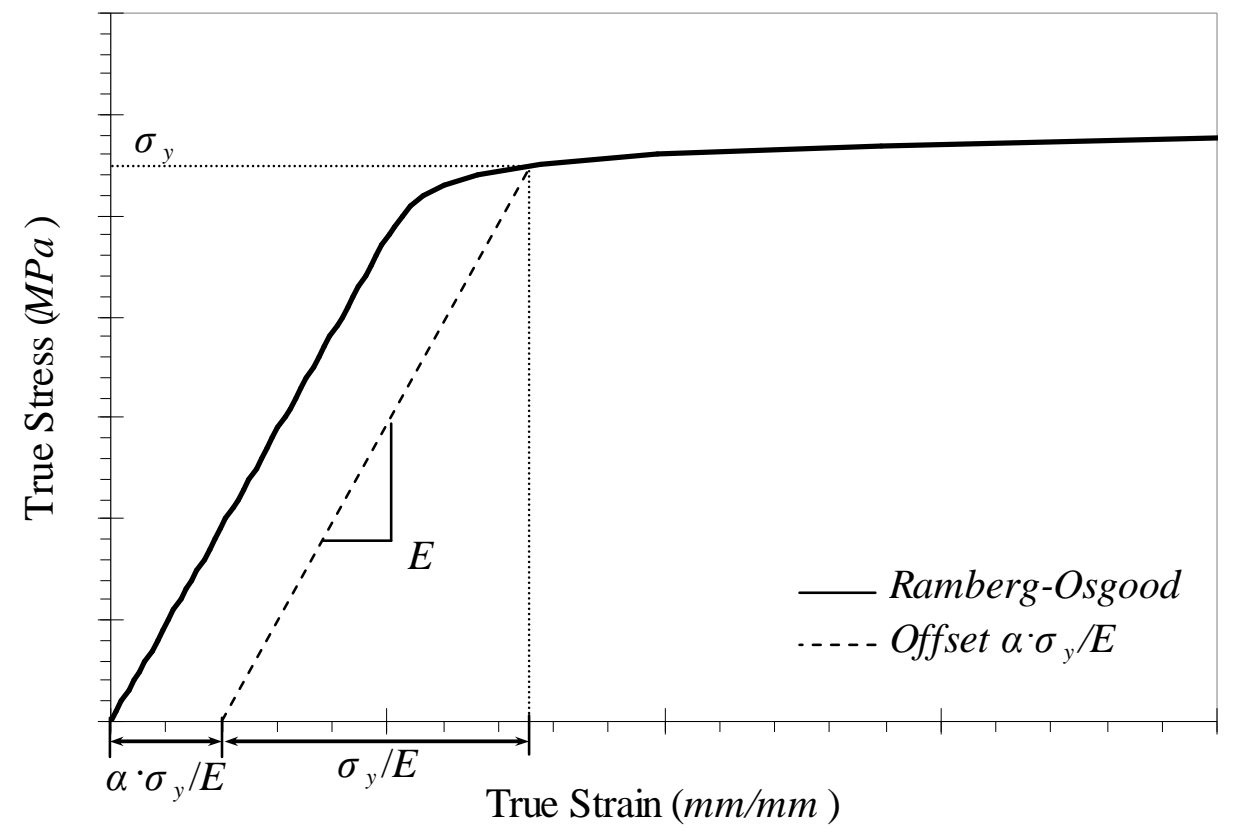

Figura 3.8. Curva tensión-deformación típica del modelo de Ramberg Osgood.

\subsubsection{Análisis elastoplástico por EF}

Como se ha visto anteriormente, para la evaluación de $P_{U}$ en las probetas SPT prefisuradas es necesaria la simulación numérica directa de dichas probetas. Este procedimiento requiere la realización de un cálculo elastoplástico con contactos. El software elegido para la realización de esta tarea ha sido el programa MSC.Marc [98].

Debido a la simetría que presenta el SPT con probeta prefisurada se ha modelizado un cuarto de la geometría en 3D con elementos hexaédricos de 8 nodos (HEX 8), utilizando los planos YZ y ZX como planos de simetría, como se puede ver en la Figura 3.9. El punzón y las matrices inferior y superior se han modelado como superficies rígidas. El cálculo se ha controlado a través del desplazamiento del punzón, imponiendo un valor de éste, similar al de 
los ensayos SPT experimentales para cada tamaño de probeta, pudiéndose considerar ambos cuasiestáticos.

El material de la probeta se ha considerado como elasto-plástico, implementándolo en la simulación gracias al modelo de plasticidad denominado "Piecewise Linear", el cuál realiza una interpolación lineal entre los puntos que definen la zona plástica de la curva tensióndeformación verdadera del material.

Para definir dichos puntos se han utilizado los datos experimentales de la curva tensióndeformación, obtenidos en el apartado 4.3 de la presente Tesis, pudiendo además, ajustar los parámetros de la ecuación (3.5) del modelo de comportamiento de material de Ramberg Osgood, los cuales son el módulo de Young $(E)$, el límite elástico del material para una deformación plástica del $0.2 \%\left(\sigma_{0.2}\right)$ y el exponente de endurecimiento $n$. Si no se poseen los datos de la curva tensión-deformación el comportamiento elastoplástico del material se puede estimar según el procedimiento que se recoge en el apartado 4.4. Esta modelización por EF es completamente válida para cualquier tamaño de probeta y profundidad de fisura $a$.

Una vez realizado el cálculo se procede a evaluar la carga para la que se alcanza la deformación plástica generalizada en la sección neta central del plano de fisura de la probeta, la cuál corresponde a la parte que no está empotrada por las matrices superior e inferior, como se puede ver en la Figura 3.9, coincidiendo con el área del rectángulo punteado de la figura. El valor de esta carga se considerará como $P_{U}$ cuando la deformación plástica equivalente alcance el valor de 0.002 en todos los puntos de la sección neta central.

Desde un punto de vista práctico, y para la posible aplicación ingenieril de la metodología propuesta, sería conveniente evitar la realización del cálculo elastoplástico comentado cada vez que se desee aplicar dicha metodología, lo cuál supondría una considerable reducción de los recursos de software necesarios y del tiempo de aplicación. Para tal fin, en la presente Tesis se han utilizado las técnicas de superficie de respuesta basadas en el diseño de experimentos, las cuáles permiten obtener una serie de funciones con las que estimar el valor de $P_{U}$ de una forma directa, para cualquier tipo de acero cuya ley de comportamiento se ajuste a una ecuación tipo Ramberg Osgood, siempre y cuando sus parámetros característicos estén dentro del rango de valores aquí considerados. 

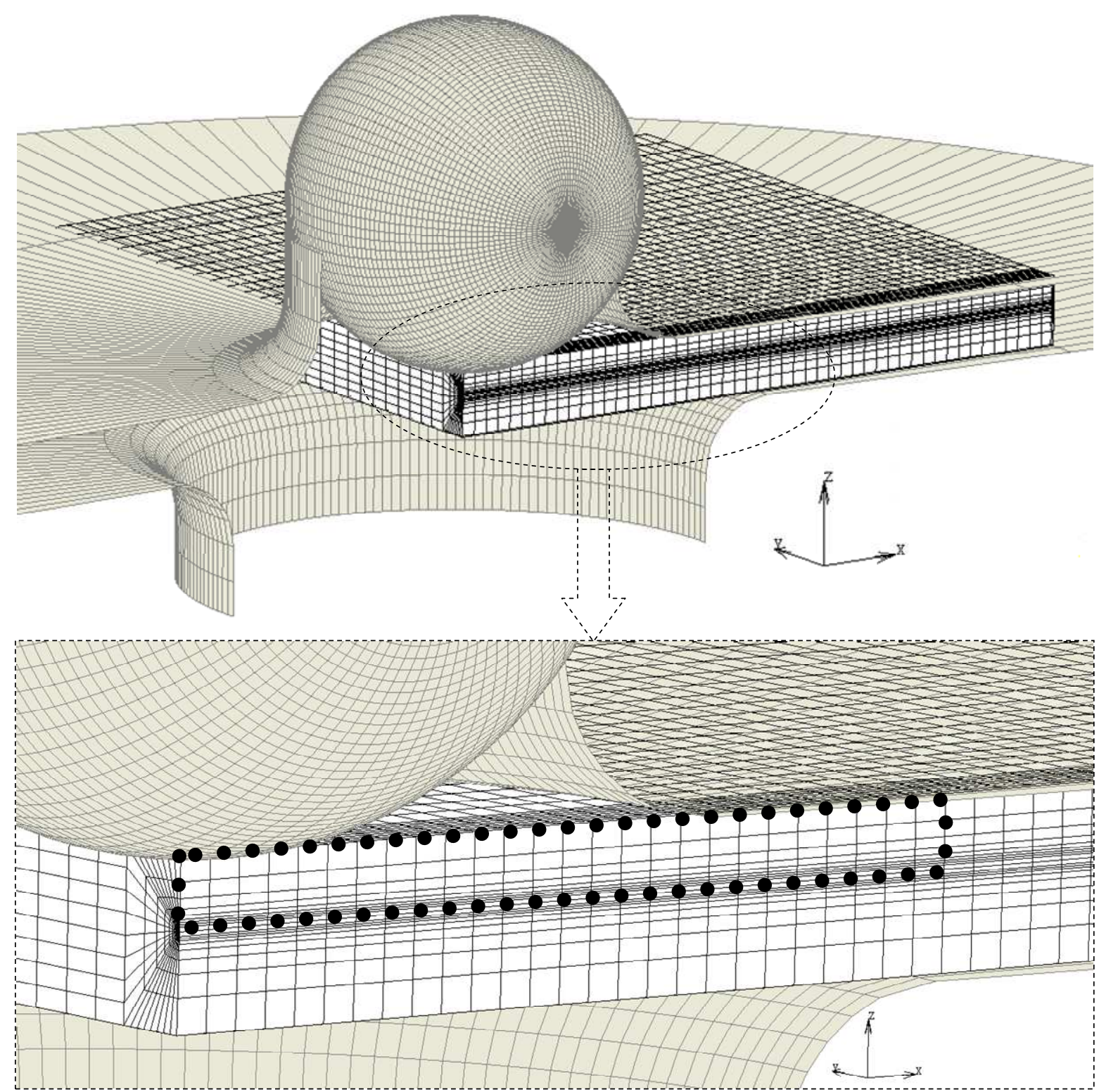

Figura 3.9. Modelado de un cuarto de probeta SPT prefisurada y detalle de la sección neta central del plano de fisura.

\subsubsection{Superficie de respuesta de $P_{U}$}

Antes de proceder a la determinación de estas superficies de respuesta es necesario apuntar una primera reflexión sobre los parámetros que influyen en el valor de $P_{U}$. Dichos parámetros se pueden englobar en dos grandes grupos, que denominaremos parámetros elastoplásticos del material y parámetros geométricos. Todos estos parámetros aparecen recogidos en la Tabla 3.2. 
Tabla 3.2. Parámetros influyentes en el valor de $P_{U}$.

\begin{tabular}{|c|c|}
\hline $\begin{array}{c}\text { Parámetros elastoplásticos } \\
\text { del material }\end{array}$ & Parámetros geométricos \\
\hline$E$ & Profundidad fisura $(a)$ \\
\hline$v$ & Dimensiones probeta $(b \times b \times t)$ \\
\hline$\sigma_{0.2}$ & Diámetro del punzón $(d)$ \\
\hline$n$ & Diámetro matriz inferior $\left(d_{m}\right)$ \\
\hline & Radio de acuerdo matriz inf. $(r)$ \\
\hline
\end{tabular}

Resulta evidente que es extremadamente complicado obtener una superficie de respuesta que englobe la variación de todos estos parámetros, por lo que es necesario fijar algunos de ellos. Siguiendo un criterio de economía de costes, resulta justificado fijar los parámetros geométricos, dependientes del utillaje, así como el espesor de probeta asignado a cada utillaje.

La profundidad de la fisura inicial $a$, es una dimensión claramente variable no solo por las propias variaciones asociadas al mecanizado de la misma, sino también porque su tamaño constituye uno de los parámetros fundamentales del proceso de fractura. Uno de los logros de la presente Tesis es la determinación del tamaño de fisura óptimo para la aplicación de la metodología desarrollada. Las funciones calculadas han sido obtenidas para dos tipos de

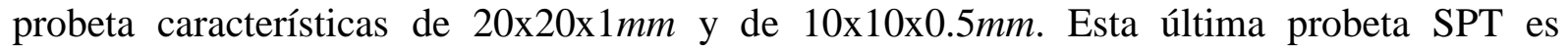
típicamente utilizada en la mayoría de los casos en los que se hace uso del SPT.

De los parámetros elastoplásticos del material el único que se ha fijado ha sido el coeficiente de Poisson, cuyo efecto en el valor de $P_{U}$ se ha comprobado irrelevante, mientras que a los demás se los ha hecho variar entre los valores típicos para los aceros. La Tabla 3.3 y la Tabla 3.4 recogen tanto los intervalos de valores considerados como los valores fijos de los parámetros con los que se ha trabajado para la obtención de la superficie de respuesta de $P_{U}$, para las probetas de 20x20x1mm y de 10x10x0.5mm respectivamente. Se puede observar como el rango de variación de los parámetros variables es el mismo en ambos tamaños de probeta, este hecho permitirá más adelante plantear un único diseño de experimentos con el que obtener la superficie de respuesta de $P_{U}$. 
Tabla 3.3. Parámetros considerados en el cálculo de $P_{U}$ para la probeta $20 \times 20 \times 1 \mathrm{~mm}$.

\begin{tabular}{|l|c|l|c|}
\hline \multicolumn{2}{|c|}{ Parámetros fijos } & \multicolumn{2}{c|}{ Parámetros variables } \\
\hline$v$ & 0.29 & $E(M P a)$ & {$[185000,215000]$} \\
\hline Dimensiones probeta $(m m x m m x m m)$ & $20 \times 20 \times 1$ & $\sigma_{0.2}(M P a)$ & {$[200,1400]$} \\
\hline Diámetro del punzón, $d(m m)$ & 5 & $n$ & {$[4,50]$} \\
\hline Diámetro matriz inferior, $d_{m}(\mathrm{~mm})$ & 8 & $a / t$ & $0.5,0.4,0.3,0.2$ \\
\hline Radio de acuerdo matriz inf., $r(\mathrm{~mm})$ & 1 & & \\
\hline
\end{tabular}

Tabla 3.4. Parámetros considerados en el cálculo de $P_{U}$ para la probeta $10 \times 10 \times 0.5 \mathrm{~mm}$.

\begin{tabular}{|l|c|l|c|}
\hline \multicolumn{2}{|c|}{ Parámetros fijos } & \multicolumn{2}{c|}{ Parámetros variables } \\
\hline$v$ & 0.29 & $E(M P a)$ & {$[185000,215000]$} \\
\hline Dimensiones probeta $(m m x m m x m m)$ & $10 \times 10 \times 0.5$ & $\sigma_{0.2}(M P a)$ & {$[200,1400]$} \\
\hline Diámetro del punzón, $d(m m)$ & 2.5 & $n$ & {$[4,50]$} \\
\hline Diámetro matriz inferior, $d_{m}(\mathrm{~mm})$ & 4 & $a / t$ & $0.5,0.4,0.3,0.2$ \\
\hline Radio de acuerdo matriz inf., $r(\mathrm{~mm})$ & 0.5 & & \\
\hline
\end{tabular}

Se puede observar en las tablas anteriores, como a pesar de haber fijado un considerable número de parámetros sigue habiendo cuatro parámetros variables para cada tamaño de probeta. Suponiendo que para la aplicación de la metodología se considera una probeta en la que la relación $a / t$ es de 0.5 , los parámetros variables se reducen de nuevo de cuatro a tres, con lo que la obtención de la superficie de respuesta de $P_{U}$ se convierte en factible.

Los parámetros $E, \sigma_{0.2}$ y $n$ son los que van a gobernar la superficie de respuesta de $P_{U}$ ya que los demás permanecen fijos. La relación existente entre estos tres parámetros y el valor de $P_{U}$ se puede expresar como $P_{U}=f\left(E^{*}, \sigma_{0.2}^{*}, n^{*}\right)$, donde $f$ se postula como modelo cuadrático de la forma expresada en la ecuación (3.6), donde $E^{*}, \sigma_{0.2}^{*}$ y $n^{*}$ son las variables codificadas de $E, \sigma_{0.2}$ y $n$ respectivamente. La codificación de los valores reales de los parámetros es necesaria para que todos ellos varíen en el mismo intervalo, como se puede ver 
en la Figura 3.10, favoreciendo de esta manera la estimación precisa de los coeficientes que definen la función $f\left(E^{*}, \sigma_{0.2}^{*}, n^{*}\right)$. Para cualquier valor real $X_{i}$ de los parámetros variables dicha codificación se puede realizar a través de la expresión (3.7), obteniendo el valor codificado $x_{i}$ correspondiente. Donde $X_{i N I n f}$ es el valor real del nivel más bajo del factor $i$, $X_{i N S u p}$ es el valor real del nivel más alto del factor $i, \mathrm{y} \tilde{X}_{i}$ es la media entre los valores reales del nivel más alto y más bajo del factor $i$.

$$
\begin{gathered}
\begin{array}{c}
f\left(E^{*}, \sigma_{0.2}^{*}, n^{*}\right)= \\
+b_{0}+b_{1} E^{*}+b_{2} \sigma_{0.2}^{*}+b_{3} n^{*}+b_{11} E^{* 2}+b_{22} \sigma_{0.2}^{* 2}+b_{33} n^{* 2}+ \\
+b_{12} E^{*} \sigma_{0.2}^{*}+b_{13} E^{*} n^{*}+b_{23} \sigma_{0.2}^{*} n^{*}
\end{array} \\
x_{i}=\frac{2 \cdot 1.682 \cdot\left(X_{i}-\tilde{X}_{i}\right)}{X_{i N \text { Sup }}-X_{i N I n f}} \quad i=E^{*}, \sigma_{0.2}^{*}, n^{*}, \frac{a^{*}}{t}
\end{gathered}
$$

La determinación de los coeficientes de la función $f\left(E^{*}, \sigma_{0.2}^{*}, n^{*}\right)$ para cada tamaño de probeta se va a realizar a través de un diseño de experimentos central compuesto [99-101], utilizando el software NEMRODW [102] para la estimación de dichos coeficientes. Las principales características de este diseño son:

- Utilización de tres factores, los cuáles son los parámetros $E, \sigma_{0.2}$ y $n$.

- Dominio esférico de radio clásico, el valor del radio para tres factores es 1.682.

- Codificación de los intervalos de valores de los tres factores recogidos en la Tabla 3.3 y en la Tabla 3.4 para que varíen en [-1.682,1.682], según la expresión (3.7).

- Matriz de experimentos composite, la cuál tiene cinco niveles para cada factor, como se puede comprobar en la Figura 3.10.

- Sin repeticiones en el punto central del dominio ( $\mathrm{N}^{\mathrm{o}}$ de experimento 15$)$, ya que no tiene sentido al utilizar la simulación numérica para obtener el valor de $P_{U}$.

- Utilización de un modelo cuadrático, definido por la expresión (3.6), para el ajuste de la superficie de respuesta. 


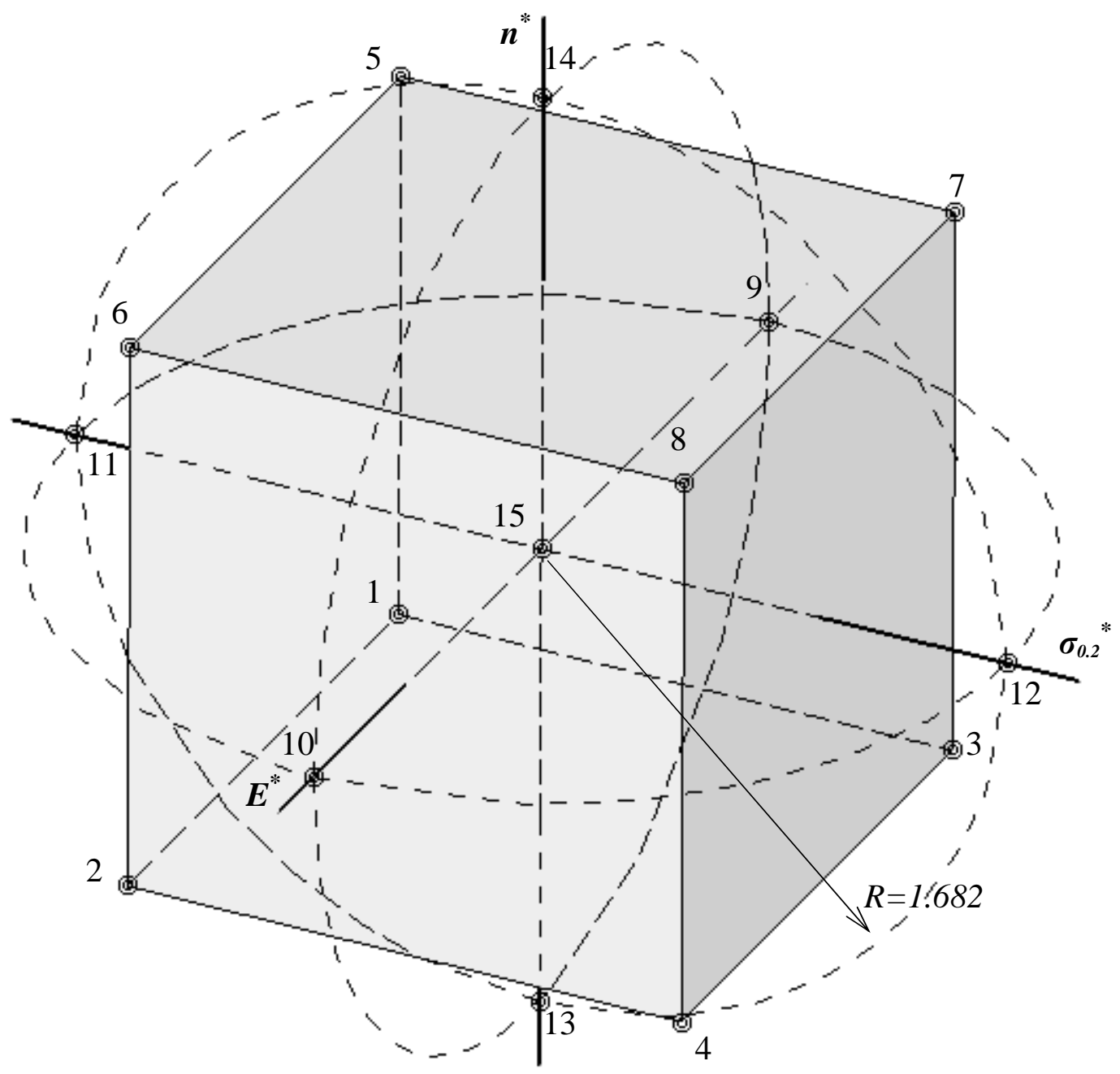

Figura 3.10. Representación de la matriz de experimentos según las variables codificadas.

La Tabla 3.5 recoge, tanto la matriz de experimentos con las variables codificadas, como la matriz del plan de experimentación con los valores de los factores del diseño de experimentos propuesto para la determinación de los coeficientes de la función $f\left(E^{*}, \sigma_{0.2}^{*}, n^{*}\right)$. Por utilizarse el mismo rango de valores para los parámetros $E, \sigma_{0.2}$ y $n$ con las dos dimensiones de probeta, los valores de dicha tabla son válidos en ambos casos.

Cada uno de los experimentos del plan de experimentación se realiza a través de una simulación numérica tal y como se recoge en el apartado 3.4.2.1 de la presente Tesis, con la única salvedad que los valores de $E, \sigma_{0.2}$ y $n$ son los recogidos en la Tabla 3.5, obteniendo como resultado final del experimento el valor de $P_{U}$. La Tabla 3.6 recoge los valores de la 
respuesta de $P_{U}$ obtenidos en cada una de las simulaciones que conforman el plan de experimentación para cada tamaño de probeta.

Tabla 3.5. Diseño propuesto para obtener la superficie de respuesta de $P_{U}$.

\begin{tabular}{|c|c|c|c|c|c|c|}
\cline { 2 - 7 } \multicolumn{1}{c|}{} & \multicolumn{3}{c|}{$\begin{array}{c}\text { Matriz de experimentos } \\
\text { (Variables codificadas) }\end{array}$} & \multicolumn{2}{c|}{$\begin{array}{c}\text { Plan de experimentación } \\
\text { (Variables reales) }\end{array}$} \\
\hline $\mathrm{N}^{\circ}$ Exp. & $E^{*}$ & $\sigma_{0.2}^{*}$ & $n^{*}$ & $E(M P a)$ & $\sigma_{0.2}(M P a)$ & $n$ \\
\hline 1 & -1 & -1 & -1 & 191082 & 443 & 13.33 \\
\hline 2 & 1 & -1 & -1 & 208918 & 443 & 13.33 \\
\hline 3 & -1 & 1 & -1 & 191082 & 1157 & 13.33 \\
\hline 4 & 1 & 1 & -1 & 208918 & 1157 & 13.33 \\
\hline 5 & -1 & -1 & 1 & 191082 & 443 & 40.67 \\
\hline 6 & 1 & -1 & 1 & 208918 & 443 & 40.67 \\
\hline 7 & -1 & 1 & 1 & 191082 & 1157 & 40.67 \\
\hline 8 & 1 & 1 & 1 & 208918 & 1157 & 40.67 \\
\hline 9 & -1.682 & 0 & 0 & 185000 & 800 & 27 \\
\hline 10 & 1.682 & 0 & 0 & 215000 & 800 & 27 \\
\hline 11 & 0 & -1.682 & 0 & 200000 & 200 & 27 \\
\hline 12 & 0 & 1.682 & 0 & 200000 & 1400 & 27 \\
\hline 13 & 0 & 0 & -1.682 & 200000 & 800 & 4 \\
\hline 14 & 0 & 0 & 1.682 & 200000 & 800 & 50 \\
\hline 15 & 0 & 0 & 0 & 200000 & 800 & 27 \\
\hline & & & & & & \\
\hline
\end{tabular}

Una vez obtenidos estos valores, con la ayuda de NEMRODW, es posible determinar los coeficientes de la función $f\left(E^{*}, \sigma_{0.2}^{*}, n^{*}\right)$, los cuales se muestran en la Tabla 3.7 en función del tamaño de probeta utilizado. Al 99\% de confianza los coeficientes significativos para la función $f\left(E^{*}, \sigma_{0.2}^{*}, n^{*}\right)$ son los que se han marcado con asteriscos en función del grado de significación, siendo los más significativos los coeficientes $b_{0}$ y $b_{2}$ en ambos casos.

Los coeficientes que no son significativos contribuyen a curvar correctamente la superficie de respuesta, por lo que no es conveniente eliminarlos de la función $f\left(E^{*}, \sigma_{0.2}^{*}, n^{*}\right)$. En ambos casos, la regresión es muy significativa estadísticamente (p-valor $<10^{-4}$ ) y explica el $99.6 \%$ de la varianza de $P_{U}$. 
Analizando la Tabla 3.7 podemos concluir que el parámetro más influyente en el valor de $P_{U}$ es $\sigma_{0.2}$, donde un aumento de este supondría un aumento de $P_{U}$, ya que el coeficiente $b_{2}$ es positivo. El segundo parámetro influyente es el coeficiente $n$, donde un aumento de este equivaldría a una disminución en el valor de $P_{U}$, ya que el coeficiente $b_{3}$ es negativo. El parámetro $E$ no es relevante en el valor de $P_{U}$ ya que su coeficiente $b_{1}$ no es significativo. Esto nos permitiría fijar el valor de $E$, por ejemplo, al valor central del dominio en el caso de no conocer su valor en el acero que se este analizando. Por último, se podría comentar que ni las interacciones entre los parámetros ni su efecto al cuadrado son significativos en el valor de $P_{U} \cdot$

A partir de la función $f\left(E^{*}, \sigma_{0.2}^{*}, n^{*}\right)$ que ajusta adecuadamente los valores de $P_{U}$ obtenidos en el diseño, es posible determinar el valor de $P_{U}$ para cualquier combinación de valores de los parámetros $E, \sigma_{0.2}$ y $n$, previa codificación, siempre y cuando nos encontremos dentro del dominio esférico definido anteriormente para este diseño. Las expresiones (3.8) y (3.9) representan la superficie de respuesta de $P_{U}$ obtenida a partir de los coeficientes de la Tabla 3.7 para los dos tamaños de probeta utilizados. Como se ha comentado en otras ocasiones, para la determinación de los parámetros $E, \sigma_{0.2}$ y $n$ se pueden utilizar los datos experimentales de la curva tensión-deformación o estimarlos a partir de los ensayos SPT.

$$
\begin{aligned}
f\left(E^{*}, \sigma_{0.2}^{*}, n^{*}\right)_{20 \times 20 x 1}= & 2.899-0.013 E^{*}+1.468 \sigma_{0.2}^{*}-0.134 n^{*}-0.02 E^{* 2}+0.069 \sigma_{0.2}^{* 2}+ \\
+ & 0.106 n^{* 2}-0.015 E^{*} \sigma_{0.2}^{*}-0.015 E^{*} n^{*}-0.04 \sigma_{0.2}^{*} n^{*} \\
f\left(E^{*}, \sigma_{0.2}^{*}, n^{*}\right)_{10 x 10 x 0.5}= & 0.734-0.003 E^{*}+0.366 \sigma_{0.2}^{*}-0.034 n^{*}-0.008 E^{* 2}+0.015 \sigma_{0.2}^{* 2}+ \\
& +0.024 n^{* 2}-0.006 E^{*} \sigma_{0.2}^{*}-0.004 E^{*} n^{*}-0.006 \sigma_{0.2}^{*} n^{*}
\end{aligned}
$$

Comparando los coeficientes significativos de la función que define la superficie de respuesta para la probeta de dimensiones 20x20x1 $\mathrm{mm}$ recogidos en la Tabla 3.7, con los coeficientes significativos de la función que define la superficie de respuesta para la probeta de dimensiones 10x10x0.5mm recogidos en esa misma tabla, se puede determinar que los coeficientes significativos de la primera función son aproximadamente cuatro veces mayores que los de la segunda, lo mismo sucede con los valores de $P_{U}$ de la Tabla 3.6, los cuáles podrían relacionarse del mismo modo. 
Tabla 3.6. Valores de la respuesta obtenida de $P_{U}$.

\begin{tabular}{|c|c|c|c|c|c|}
\cline { 2 - 6 } \multicolumn{1}{c|}{} & \multicolumn{2}{c|}{$\begin{array}{c}\text { Plan de experimentación } \\
\text { (Variables reales) }\end{array}$} & $\begin{array}{c}\text { Respuesta } \\
\text { probeta } \\
\text { 20x20x1mm }\end{array}$ & $\begin{array}{c}\text { Respuesta } \\
\text { probeta } \\
\text { 10x10x0.5mm }\end{array}$ \\
\hline $\mathrm{N}^{\circ}$ Exp. & $E(M P a)$ & $\sigma_{0.2}(M P a)$ & $n$ & $P_{U}(k N)$ & $P_{U}(k N)$ \\
\hline 1 & 191082 & 443 & 13.33 & 1.58 & 0.40 \\
\hline 2 & 208918 & 443 & 13.33 & 1.58 & 0.41 \\
\hline 3 & 191082 & 1157 & 13.33 & 4.59 & 1.15 \\
\hline 4 & 208918 & 1157 & 13.33 & 4.58 & 1.14 \\
\hline 5 & 191082 & 443 & 40.67 & 1.53 & 0.38 \\
\hline 6 & 208918 & 443 & 40.67 & 1.52 & 0.38 \\
\hline 7 & 191082 & 1157 & 40.67 & 4.43 & 1.11 \\
\hline 8 & 208918 & 1157 & 40.67 & 4.31 & 1.08 \\
\hline 9 & 185000 & 800 & 27 & 2.91 & 0.73 \\
\hline 10 & 215000 & 800 & 27 & 2.89 & 0.72 \\
\hline 11 & 200000 & 200 & 27 & 0.67 & 0.17 \\
\hline 12 & 200000 & 1400 & 27 & 5.63 & 1.41 \\
\hline 13 & 200000 & 800 & 4 & 3.64 & 0.91 \\
\hline 14 & 200000 & 800 & 50 & 2.87 & 0.72 \\
\hline 15 & 200000 & 800 & 27 & 2.88 & 0.73 \\
\hline
\end{tabular}

Tabla 3.7. Coeficientes de la función $f\left(E, \sigma_{0.2}, n\right)$ (variables codificadas).

\begin{tabular}{|c|c|c|c|c|}
\cline { 2 - 5 } \multicolumn{1}{c|}{} & \multicolumn{2}{c|}{$\begin{array}{c}\text { Probeta } \\
\text { 20x20x1mm }\end{array}$} & \multicolumn{2}{c|}{$\begin{array}{c}\text { Probeta } \\
\text { 10x10x0.5mm }\end{array}$} \\
\hline Coeficiente & Valor & Significación (\%) & Valor & Significación (\%) \\
\hline$b_{0}$ & 2.899 & $<0.01 * * *$ & 0.734 & $<0.01 * * *$ \\
\hline$b_{1}$ & -0.013 & 77.4 & -0.003 & 74.0 \\
\hline$b_{2}$ & 1.468 & $<0.01 * * *$ & 0.366 & $<0.01 * * *$ \\
\hline$b_{3}$ & -0.134 & $2.39 *$ & -0.034 & $1.70^{*}$ \\
\hline$b_{11}$ & -0.02 & 76.9 & -0.008 & 61.2 \\
\hline$b_{22}$ & 0.069 & 32.4 & 0.015 & 35.3 \\
\hline$b_{33}$ & 0.106 & 15.3 & 0.024 & 16.5 \\
\hline$b_{12}$ & -0.015 & 79.5 & -0.006 & 64.5 \\
\hline$b_{13}$ & -0.015 & 79.5 & -0.004 & 78.1 \\
\hline$b_{23}$ & -0.04 & 49.8 & -0.006 & 64.5 \\
\hline
\end{tabular}


Considerando que el factor de forma existente es que los parámetros geométricos de la primera probeta $(20 \times 20 \times 1 \mathrm{~mm})$ son el doble que los parámetros geométricos de la segunda probeta $(10 \times 10 \times 0.5 \mathrm{~mm})$ se produce una ambigüedad en la relación entre los valores de $P_{U}$ y el factor de forma, es decir, en este caso concreto se obtiene el mismo resultado al considerar que la relación existente entre los valores de $P_{U}$ es el doble del factor de forma $(2 \times 2=4)$, o bien, es el cuadrado del factor de forma $\left(2^{2}=4\right)$. Para dilucidar esta ambigüedad se ha optado por calcular el valor de $P_{U}$ para cada uno de los experimentos que conforman el diseño de experimentos recogido en la Tabla 3.5 en una probeta de dimensiones 40x40x2mm, a la cuál la corresponde un factor de forma de 4 con respecto a la probeta de $10 \mathrm{x} 10 \mathrm{x} 0.5 \mathrm{~mm}$ y un factor de forma de 2 con respecto a la probeta de 20x20x1 $\mathrm{mm}$. La Tabla 3.8 recoge los valores obtenidos de $P_{U}$ mediante simulación numérica para los tres tamaños de probeta utilizados, así como la relación existente para cada experimento entre el valor de $P_{U}$ de una de las probetas con respecto a los valores de $P_{U}$ de las otras dos dimensiones de probeta.

Con los valores medios de las relaciones entre los valores de $P_{U}$ queda clarificada la ambigüedad presentada, siendo el cuadrado del factor de forma la relación existente entre los valores de $P_{U}$ tal y como se recoge en la expresión (3.10).

$P_{U M}=\Delta_{M / m}^{2} \cdot P_{U m}$

Donde $P_{U M}$ es la carga de colapso plástico correspondiente a la probeta de mayor dimensión, $P_{U m}$ es la carga de colapso plástico correspondiente a la probeta de menor dimensión y $\Delta_{M / m}$ es el factor de forma existente entre la probeta de mayor y menor dimensión consiguiendo de esta manera que siempre sea mayor que la unidad. En el supuesto de invertir el orden entre las probetas, es decir, que $P_{U M}$ corresponda a la probeta de menor dimensión y $P_{U m}$ a la de mayor dimensión, el factor de forma $\left(\Delta_{M / m}\right)$ tomará un valor menor que la unidad.

La relación establecida será de gran ayuda en aquellos casos en los que se desee estimar la carga de colapso plástico a partir del valor conocido de $P_{U}$ correspondiente a una probeta de diferentes dimensiones, siempre y cuando se cumplan las condiciones expresadas anteriormente. 
Tabla 3.8. Relación existente entre los valores de $P_{U}$ para cada dimensión de probeta.

\begin{tabular}{|c|c|c|c|c|c|c|}
\hline \multirow[b]{2}{*}{$\mathrm{N}^{\circ}$ Exp. } & \multicolumn{3}{|c|}{$P_{U}(k N)$} & \multirow{2}{*}{$\frac{P_{U 40 \times 40 \times 2}}{P_{U 10 \times 10 \times 0.5}}$} & \multirow{2}{*}{$\frac{P_{U 40 \times 40 \times 2}}{P_{U 20 \times 20 \times 1}}$} & \multirow{2}{*}{$\frac{P_{U 20 \times 20 \times 1}}{P_{U 10 \times 10 \times 0.5}}$} \\
\hline & $10 \times 10 \times 0.5$ & $20 \times 20 \times 1$ & $40 \times 40 \times 2$ & & & \\
\hline 1 & 0.40 & 1.58 & 6.33 & 15.99 & 4.00 & 3.99 \\
\hline 2 & 0.41 & 1.58 & 6.27 & 15.46 & 3.98 & 3.88 \\
\hline 3 & 1.15 & 4.59 & 18.35 & 16.00 & 4.00 & 4.00 \\
\hline 4 & 1.14 & 4.58 & 18.27 & 15.97 & 3.99 & 4.00 \\
\hline 5 & 0.38 & 1.53 & 6.12 & 16.00 & 4.00 & 4.00 \\
\hline 6 & 0.38 & 1.52 & 6.05 & 15.96 & 3.99 & 4.00 \\
\hline 7 & 1.11 & 4.43 & 17.71 & 15.99 & 4.00 & 4.00 \\
\hline 8 & 1.08 & 4.31 & 17.25 & 15.99 & 4.00 & 4.00 \\
\hline 9 & 0.73 & 2.91 & 11.65 & 16.00 & 4.00 & 4.00 \\
\hline 10 & 0.72 & 2.89 & 11.54 & 15.98 & 4.00 & 4.00 \\
\hline 11 & 0.17 & 0.67 & 2.69 & 15.98 & 4.00 & 4.00 \\
\hline 12 & 1.41 & 5.63 & 22.52 & 16.00 & 4.00 & 4.00 \\
\hline 13 & 0.91 & 3.64 & 14.55 & 16.00 & 4.00 & 4.00 \\
\hline 14 & 0.72 & 2.87 & 11.48 & 15.99 & 4.00 & 4.00 \\
\hline 15 & 0.73 & 2.88 & 11.51 & 15.84 & 4.00 & 3.96 \\
\hline & & & Media & 15.94 & 4.00 & 3.99 \\
\hline & & & $\Delta_{M / m}$ & 4 & 2 & 2 \\
\hline
\end{tabular}

Retomando el hecho de la falta de significación del módulo de elasticidad $E$ en la función $f\left(E, \sigma_{0.2}, n\right)$ y si además se tiene en cuenta el proceso de fisuración por láser para obtener la fisura inicial, en el que hay que verificar la profundidad real de la fisura posteriormente al ensayo de la probeta, ya que a priori, no se conoce cuantitativamente la diferencia que puede existir entre la profundidad de fisura teórica y la real, parece obvio por todo esto, que la determinación de una superficie de respuesta de $P_{U}$ que incluya la posible variación de la profundidad de fisura inicial, aportaría mayor precisión en la estimación de $P_{U}$. La variación de $a$ se va a tener en cuenta a través de la relación adimensional $a / t$. De esta forma el intervalo de variación de esta relación servirá para cualquier tamaño de probeta. Por ello, en 
las siguientes líneas se va a proceder a determinar una superficie de respuesta de $P_{U}$, para los dos tamaños de probeta utilizados anteriormente, en la que los parámetros variables sean $a / t$, $\sigma_{0.2}$ y $n$.

La Tabla 3.9 y la Tabla 3.10 recogen tanto los valores fijos como el intervalo de valores de los parámetros con los que se obtiene la superficie de respuesta de $P_{U}$, aquí expuesta, para las probetas de 20x20x1 $\mathrm{mm}$ y de $10 \times 10 \times 0.5 \mathrm{~mm}$ respectivamente.

Tabla 3.9. Parámetros influyentes en el valor de $P_{U}$ para la probeta $20 \times 20 \times 1 \mathrm{~mm}$.

\begin{tabular}{|l|c|l|c|}
\hline \multicolumn{2}{|c|}{ Parámetros fijos } & \multicolumn{2}{c|}{ Parámetros variables } \\
\hline$v$ & 0.29 & $a / t$ & {$[0.1,0.7]$} \\
\hline Dimensiones probeta $(m m x m m x m m)$ & $20 \times 20 \times 1$ & $\sigma_{0.2}(M P a)$ & {$[200,1400]$} \\
\hline Diámetro del punzón, $d(m m)$ & 5 & $n$ & {$[4,50]$} \\
\hline Diámetro matriz inferior, $d_{m}(\mathrm{~mm})$ & 8 & & \\
\hline Radio de acuerdo matriz inf., $r(\mathrm{~mm})$ & 1 & & \\
\hline$E(M P a)$ & 200000 & & \\
\hline
\end{tabular}

Tabla 3.10. Parámetros influyentes en el valor de $P_{U}$ para la probeta $10 \times 10 \times 0.5 \mathrm{~mm}$.

\begin{tabular}{|l|c|l|c|}
\hline \multicolumn{2}{|c|}{ Parámetros fijos } & \multicolumn{2}{c|}{ Parámetros variables } \\
\hline$v$ & 0.29 & $a / t$ & {$[0.1,0.7]$} \\
\hline Dimensiones probeta $(m m x m m x m m)$ & $10 \times 10 \times 0.5$ & $\sigma_{0.2}(M P a)$ & {$[200,1400]$} \\
\hline Diámetro del punzón, $d(m m)$ & 2.5 & $n$ & {$[4,50]$} \\
\hline Diámetro matriz inferior, $d_{m}(\mathrm{~mm})$ & 4 & & \\
\hline Radio de acuerdo matriz inf., $r(\mathrm{~mm})$ & 0.5 & & \\
\hline$E(M P a)$ & 200000 & & \\
\hline
\end{tabular}

Los valores del intervalo de variación de $\sigma_{0.2}$ y $n$ siguen siendo los mismos con los que se ha trabajado anteriormente, mientras que el intervalo de variación de la relación $a / t$ está entre 0.1 que representaría una fisura inicial de poca profundidad, y 0.7 que caracterizaría una fisura inicial prácticamente pasante. 
Al igual que sucedía anteriormente, los parámetros variables son los que van a gobernar la superficie de respuesta de $P_{U}$ ya que los demás permanecen fijos, con la única salvedad de que el parámetro $E$ se ha cambiado por $a / t$. La relación existente entre estos tres parámetros variables $a / t, \sigma_{0.2} \mathrm{y} n$, y el valor de $P_{U}$ se puede expresar como $P_{U}=g\left(a / t^{*}, \sigma_{0.2}^{*}, n^{*}\right)$, donde $g$ se postula como modelo cuadrático de la forma expresada en la ecuación (3.11) para las variables codificadas.

$$
\begin{aligned}
g\left(\frac{a}{t}^{*}, \sigma_{0.2}^{*}, n^{*}\right) & =b_{0}+b_{1} \frac{a^{*}}{t}+b_{2} \sigma_{0.2}^{*}+b_{3} n^{*}+b_{11} \frac{a}{t}^{* 2}+b_{22} \sigma_{0.2}^{* 2}+b_{33} n^{* 2}+ \\
& +b_{12} \frac{a^{*}}{t} \sigma_{0.2}^{*}+b_{13} \frac{a^{*}}{t} n^{*}+b_{23} \sigma_{0.2}^{*} n^{*}
\end{aligned}
$$

La determinación de los coeficientes de la función $g\left(a / t^{*}, \sigma_{0.2}^{*}, n^{*}\right)$ se ha realizado, al igual que en el caso anterior, a través de un diseño de experimentos central compuesto. La Tabla 3.11 recoge, tanto la matriz de experimentos con las variables codificadas, como la matriz del plan de experimentación con los valores de los factores del diseño de experimentos propuesto para la determinación de los coeficientes de la función $g\left(a / t^{*}, \sigma_{0.2}^{*}, n^{*}\right)$.

Por utilizarse el mismo rango de valores para los parámetros $a / t, \sigma_{0.2}$ y $n$ con las dos dimensiones de probeta, los valores de dicha tabla son válidos en ambos casos. De la misma forma que antes, cada uno de los experimentos del plan de experimentación se realiza a través de una simulación numérica, con los valores de $a / t, \sigma_{0.2}$ y $n$ establecidos en la Tabla 3.11. En esa misma tabla se incluyen los valores de la respuesta de $P_{U}$ obtenidos en cada una de las simulaciones que conforman el plan de experimentación desarrollado, para cada una de las dimensiones de probeta utilizadas.

Una vez obtenidos estos valores, se han determinado los coeficientes de la función $g\left(a / t^{*}, \sigma_{0.2}^{*}, n^{*}\right)$, los cuáles se muestran en la Tabla 3.12. Al $99 \%$ de confianza los coeficientes significativos para la función $g\left(a / t^{*}, \sigma_{0.2}^{*}, n^{*}\right)$ son los que se han marcado con asteriscos en función del grado de significación, siendo en este caso los más significativos los coeficientes $b_{0}, b_{1}$ y $b_{2}$. Al igual que antes, los coeficientes que no son significativos contribuyen a curvar correctamente la superficie de respuesta, por lo que no es conveniente 
eliminarlos de la función $g\left(a / t^{*}, \sigma_{0.2}^{*}, n^{*}\right)$. La regresión es muy significativa estadísticamente $\left(\right.$ p-valor $\left.<10^{-4}\right)$ y explica el $99.7 \%$ de la varianza de $P_{U}$.

Las conclusiones que se pueden extraer de la Tabla 3.12 son similares a las obtenidas a partir de la Tabla 3.7, destacando que el parámetro más influyente en el valor de $P_{U}$ es $\sigma_{0.2}$, donde un aumento de este supondría un aumento de $P_{U}$, ya que el coeficiente $b_{2}$ es positivo. El segundo parámetro más influyente es la relación $a / t$, donde un aumento de esta equivaldría a una disminución en el valor de $P_{U}$, ya que el coeficiente $b_{1}$ es negativo. Por último se puede reseñar, que a diferencia de lo que ocurría anteriormente, donde ni las interacciones entre los parámetros ni su efecto al cuadrado eran significativos en el valor de $P_{U}$, si que hay esta vez un coeficiente significativo, que es el $b_{12}$ correspondiente a la interacción entre $a / t$ y $\sigma_{0.2}$. Dado que su valor es negativo, un aumento del producto entre $a / t$ y $\sigma_{0.2}$ supondría una disminución del valor de $P_{U}$, sin tener en cuenta el efecto de cada uno de estos parámetros por separado.

A partir de la función $g\left(a / t^{*}, \sigma_{0.2}^{*}, n^{*}\right)$ que ajusta adecuadamente los valores de $P_{U}$ obtenidos en el diseño, es posible determinar el valor de $P_{U}$ para cualquier combinación de valores de los parámetros $a / t, \sigma_{0.2}$ y $n$, previa codificación, siempre y cuando nos encontremos dentro del dominio esférico definido anteriormente para este diseño. Las expresiones (3.12) y (3.13) representan la superficie de respuesta de $P_{U}$ obtenida a partir de los coeficientes de la Tabla 3.12 para los dos tamaños de probeta utilizados.

$$
\begin{aligned}
g\left(\frac{a^{*}}{t}, \sigma_{0.2}^{*}, n^{*}\right)_{20 x 20 x 1}= & 3.664-1.132 \frac{a^{*}}{t}+1.993 \sigma_{0.2}^{*}-0.243 n^{*}+0.13\left(\frac{a^{*}}{t}\right)^{2}+0.065 \sigma_{0.2}^{*}{ }^{2}+ \\
& +0.168 n^{* 2}-0.797 \frac{a^{*}}{t} \sigma_{0.2}^{*}+0.068 \frac{a^{*}}{t} n^{*}-0.077 \sigma_{0.2}^{*} n^{*} \\
g\left(\frac{a^{*}}{t}, \sigma_{0.2}^{*}, n^{*}\right)_{10 x 10 x 0.5}= & 0.916-0.285 \frac{a^{*}}{t}+0.498 \sigma_{0.2}^{*}-0.061 n^{*}+0.034\left(\frac{a^{*}}{t}\right)^{2}+0.016 \sigma_{0.2}^{* 2}+ \\
& +0.042 n^{* 2}-0.201 \frac{a^{*}}{t} \sigma_{0.2}^{*}+0.018 \frac{a^{*}}{t} n^{*}-0.02 \sigma_{0.2}^{*} n^{*}
\end{aligned}
$$


Tabla 3.11. Diseño propuesto para obtener la superficie de respuesta de $P_{U}$.

\begin{tabular}{|c|c|c|c|c|c|c|c|c|}
\cline { 2 - 10 } \multicolumn{1}{c|}{} & \multicolumn{3}{c|}{$\begin{array}{c}\text { Matriz de experimentos } \\
\text { (Variables codificadas) }\end{array}$} & \multicolumn{2}{c|}{$\begin{array}{c}\text { Plan de experimentación } \\
\text { (Variables reales) }\end{array}$} & $\begin{array}{c}\text { Respuesta } \\
\text { probeta } \\
\text { 20x20x1mm }\end{array}$ & $\begin{array}{c}\text { Respuesta } \\
\text { probeta } \\
\text { 10x10x0.5mm }\end{array}$ \\
\hline $\begin{array}{c}\mathrm{N}^{\circ} \\
\text { Exp. }\end{array}$ & $a / t^{*}$ & $\sigma_{0.2}^{*}$ & $n^{*}$ & $a / t$ & $\begin{array}{c}\sigma_{0.2} \\
(M P a)\end{array}$ & $n$ & $P_{U}(k N)$ & $P_{U}(k N)$ \\
\hline 1 & -1 & -1 & -1 & 0.222 & 443 & 13.33 & 2.50 & 0.62 \\
\hline 2 & 1 & -1 & -1 & 0.578 & 443 & 13.33 & 1.46 & 0.37 \\
\hline 3 & -1 & 1 & -1 & 0.222 & 1157 & 13.33 & 8.50 & 2.13 \\
\hline 4 & 1 & 1 & -1 & 0.578 & 1157 & 13.33 & 4.12 & 1.03 \\
\hline 5 & -1 & -1 & 1 & 0.222 & 443 & 40.67 & 2.28 & 0.57 \\
\hline 6 & 1 & -1 & 1 & 0.578 & 443 & 40.67 & 1.35 & 0.34 \\
\hline 7 & -1 & 1 & 1 & 0.222 & 1157 & 40.67 & 7.82 & 1.95 \\
\hline 8 & 1 & 1 & 1 & 0.578 & 1157 & 40.67 & 3.86 & 0.97 \\
\hline 9 & -1.682 & 0 & 0 & 0.1 & 800 & 27 & 5.62 & 1.41 \\
\hline 10 & 1.682 & 0 & 0 & 0.7 & 800 & 27 & 2.56 & 0.64 \\
\hline 11 & 0 & -1.682 & 0 & 0.4 & 200 & 27 & 0.78 & 0.20 \\
\hline 12 & 0 & 1.682 & 0 & 0.4 & 1400 & 27 & 7.03 & 1.76 \\
\hline 13 & 0 & 0 & -1.682 & 0.4 & 800 & 4 & 4.80 & 1.20 \\
\hline 14 & 0 & 0 & 1.682 & 0.4 & 800 & 50 & 3.59 & 0.90 \\
\hline 15 & 0 & 0 & 0 & 0.4 & 800 & 27 & 3.64 & 0.91 \\
\hline
\end{tabular}

Tabla 3.12. Coeficientes de la función $g\left(a / t^{*}, \sigma_{0.2}^{*}, n^{*}\right)$ (variables codificadas).

\begin{tabular}{|c|c|c|c|c|}
\cline { 2 - 5 } \multicolumn{1}{c|}{} & \multicolumn{2}{c|}{$\begin{array}{c}\text { Probeta } \\
\text { 20x20x1mm }\end{array}$} & \multicolumn{2}{c|}{$\begin{array}{c}\text { Probeta } \\
\text { 10x10x0.5mm }\end{array}$} \\
\hline Coeficiente & Valor & Significación $(\%)$ & Valor & Significación (\%) \\
\hline$b_{0}$ & 3.664 & $0.0273^{* * *}$ & 0.916 & $0.0261^{* * *}$ \\
\hline$b_{1}$ & -1.132 & $0.0149^{* * *}$ & -0.285 & $0.0138^{* * *}$ \\
\hline$b_{2}$ & 1.993 & $<0.01^{* * *}$ & 0.498 & $<0.01^{* * *}$ \\
\hline$b_{3}$ & -0.243 & 7.8 & -0.061 & 7.6 \\
\hline$b_{11}$ & 0.130 & 46.6 & 0.034 & 44.4 \\
\hline$b_{22}$ & 0.065 & 71.0 & 0.016 & 70.6 \\
\hline$b_{33}$ & 0.168 & 35.6 & 0.042 & 35.2 \\
\hline$b_{12}$ & -0.797 & $0.262^{* *}$ & -0.201 & $0.244 * *$ \\
\hline$b_{13}$ & 0.068 & 65.5 & 0.018 & 64.1 \\
\hline$b_{23}$ & -0.077 & 61.4 & -0.020 & 59.6 \\
\hline
\end{tabular}




\subsection{DETERMINACIÓN DE $K_{r}$ A TRAVÉS DEL DIAGRAMA FAD}

Conocido el valor de $L_{r}$ de esta forma, el siguiente paso es la obtención del ratio de tenacidad $K_{r}$, haciendo uso del diagrama FAD tal y como aparece reflejado en el gráfico de la Figura 3.3. Para la determinación de $K_{r}$ es necesaria la representación previa del diagrama FAD que se va a utilizar. Tal y como se ha visto anteriormente, existen tres opciones diferentes para obtener el diagrama de fallo en función del nivel de precisión que se desee y de los datos del material disponibles. En la presente Tesis se van a considerar las tres opciones de FAD con el fin de establecer el grado de precisión que se alcanza con la metodología desarrollada para cada una ellas. Las ecuaciones que definen las Opciones 1, 2 y 3 son las expresiones (2.14), (2.15) y (2.16) respectivamente:

Opción $1\left\{\begin{array}{lll}K_{r}=\left(1-0.14 \cdot L_{r}^{2}\right)\left[0.3+0.7 \cdot \exp \left(-0.65 \cdot L_{r}^{6}\right)\right] & \text { si } & L_{r} \leq L_{r}^{\max } \\ K_{r}=0 & \text { si } & L_{r}>L_{r}^{\max }\end{array}\right.$

Opción 2 $\left\{\begin{array}{lll}K_{r}=\left(\frac{E \cdot \varepsilon_{r e f}}{L_{r} \cdot \sigma_{0.2}}+\frac{L_{r}^{3} \cdot \sigma_{0.2}}{2 \cdot E \cdot \varepsilon_{r e f}}\right)^{-1 / 2} & \text { si } & L_{r} \leq L_{r}^{\max } \\ K_{r}=0 & \text { si } \quad L_{r}>L_{r}^{\max }\end{array}\right.$

Opción $3\left\{\begin{array}{lll}K_{r}=\left(\frac{J_{e}}{J_{a p p}}\right)^{1 / 2} & \text { si } & L_{r} \leq L_{r}^{\max } \\ K_{r}=0 & \text { si } & L_{r}>L_{r}^{\max }\end{array}\right.$

donde $L_{r}^{\max }$ viene dada por la expresión (2.13):

$$
L_{r}^{\max }=\frac{1}{2} \cdot \frac{\left(\sigma_{0.2}+\sigma_{u t s}\right)}{\sigma_{0.2}}
$$

La utilización de las expresiones (2.14) y (2.15), se puede realizar directamente, una vez conocida la curva tensión-deformación del material utilizado en el caso de la Opción 2. Sin embargo para la aplicación de la expresión (2.16), que define la Opción 3, es necesario llevar a cabo una simulación numérica para determinar los parámetros $J_{a p p}$ y $J_{e}$ de dicha expresión, 
donde $J_{a p p}$ es la integral $J$ aplicada y $J_{e}$ su parte elástica. El procedimiento para llevar a cabo dicha simulación numérica incluye necesariamente un cálculo elastoplástico con contactos. Para ello, se ha usado de nuevo el programa MSC.Marc.

Las condiciones de simetría definidas anteriormente siguen siendo válidas, por lo que únicamente se ha modelado en 3D un cuarto de probeta, utilizando elementos hexaédricos de 20 nodos (HEX 20) para lograr una mayor precisión en el cálculo de la integral $J$. En este caso, el punzón y las matrices inferior y superior también se han modelado como superficies rígidas, y la probeta como un cuerpo elastoplástico.

La integral $J$ ha sido evaluada en el entorno de la punta de fisura teniendo en cuenta toda la longitud de fisura, es en esta zona donde se producen los estados tensionales más elevados y donde se inicia la rotura. El cálculo de la integral $J$ en MSC.Marc está basado en el método del dominio de integración [98]. El volumen de integración queda definido por la línea de fisura y el camino de integración. La línea de fisura está formada por los nodos del modelo que se encuentran en toda la longitud de la punta de fisura, estos nodos en el software tienen que ser introducidos manualmente, mientras que los caminos de integración pueden ser determinados automáticamente en este caso, ya que la malla del modelo se ha generado a partir de una extrusión de elementos 2D para la obtención del modelo 3D, como se puede ver en la Figura 3.11.

En los análisis elastoplásticos como este, es ventajoso utilizar un mallado en el que haya varios nodos en la punta de fisura, dando lugar a elementos colapsados en la punta de fisura, permitiendo así que los nodos de estos elementos se puedan separar durante el cálculo, reproduciendo el enromamiento que se produce en la punta de fisura. Para este tipo de mallado existe una opción implementada en el software utilizado, denominada "multiple nodes distance", en la cuál hay que indicar el radio de la circunferencia que engloba estos nodos. Este radio siempre tiene que ser menor que el tamaño del elemento más pequeño de los que forman la punta de fisura. Todos los nodos que se encuentren dentro de esta circunferencia, con centro en los nodos previamente seleccionados de la línea de fisura, son considerados parte de la punta de fisura y el primer camino de integración estará formado por los elementos que estén conectados a estos nodos. El segundo camino de integración estará formado por los elementos que componen el primero y por todos los elementos conectados a 
ellos, y así sucesivamente con todos los caminos de integración hasta un total de nueve que es el máximo permitido por el software empleado.

Con la opción "multiple nodes distance" activada es posible modelar la punta de fisura con un pequeño redondeo inicial para evitar principalmente problemas de convergencia numérica durante el cálculo, como se puede ver en el detalle de la Figura 3.11. Una vez determinada la integral $J$ es posible obtener los parámetros $J_{a p p}$ y $J_{e}$ en cada instante del cálculo para la posterior representación del diagrama FAD según la Opción 3.

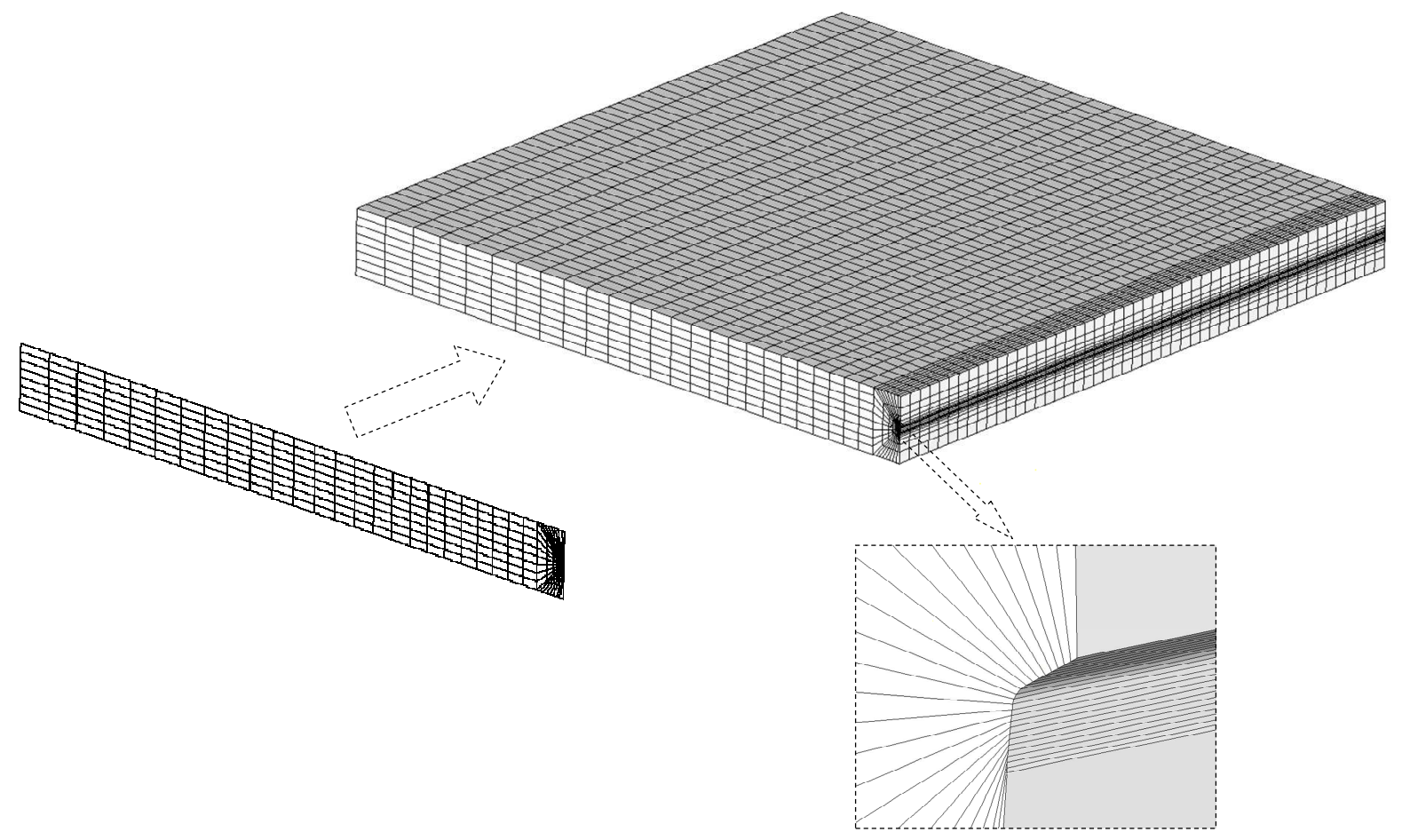

Figura 3.11. Extrusión de elementos 2D para la obtención del modelo 3D.

\subsection{EVALUACIÓN NUMÉRICA DE $K_{I}$}

Una vez representado el FAD, es posible entrar en el diagrama con el valor calculado anteriormente de $L_{r}$, ya que representa la abscisa del punto en la curva del diagrama que define el instante en el que comienza a propagar la fisura en la probeta. Hallado este punto el valor de su ordenada queda definido inmediatamente, correspondiendo este valor a $K_{r}$. Siguiendo el esquema de la Figura 3.3, el siguiente paso a realizar sería la evaluación 
numérica de los factores de intensidad de tensiones (FIT), en concreto, de $K_{I}$ haciendo uso de la simulación numérica.

Entre las técnicas más comunes para el cálculo de los FIT en modelos 3D, cabe mencionar la técnica de correlación de desplazamientos [103,104], la evaluación de la tasa de liberación de energía potencial [105], y el cálculo de la integral $J$ por medio de una integral de dominio [106]. Bittencourt [107] ha demostrado que en el caso de materiales elástico-lineales y con mallados suficientemente refinados, los tres grupos de procedimientos convergen a las mismas predicciones. En nuestro caso, se ha optado por obtener los FIT a través de la técnica de correlación de desplazamientos por tratarse de un procedimiento ventajoso desde el punto de vista computacional, además de ser un requerimiento del procedimiento de integridad estructural.

Para ello, el entorno de la fisura ha sido modelado mediante una roseta de elementos finitos concéntricamente dispuestos alrededor de la punta de la fisura, tal y como se presenta en la Figura 3.12. Se trata de elementos brick de 20 nodos, 8 en los vértices del elemento y 12 en la mitad de cada arista, que han sido convenientemente modificados para así poder reproducir las singularidades de tipo $\mathrm{r}^{-1 / 2}$ en los campos de tensiones y deformaciones. El resto de la geometría ha sido mallada con elementos brick de 20 nodos convencionales. El programa utilizado para esta simulación ha sido el código ANSYS [108]. Con este tipo de elementos el FIT en modo I puede evaluarse según la expresión (3.14),

$K_{I}=\frac{\mu}{k+1} \sqrt{\frac{2 \pi}{L}}\left[4\left(\mathrm{v}_{2}-\mathrm{v}_{4}\right)+\mathrm{v}_{5}-\mathrm{v}_{3}\right]$

siendo,

$\mu=\frac{E}{2(1+v)}$

$k=\left\{\begin{array}{cl}3-4 v & (\text { deformación plana }) \\ (3-v) /(1+v) & (\text { tensión plana })\end{array}\right.$

donde $L$ es la longitud del elemento singular $\mathrm{y}_{j}, \mathrm{v}_{i}$ son los desplazamientos nodales en las direcciones $x$ e $y$ respectivamente, donde $x$ es la dirección actual de la fisura e $y$ es la dirección 
perpendicular. Las expresiones son fácilmente demostrables a partir de las ecuaciones de los movimientos propias de la MFEL. La calidad de la técnica descrita ha sido contrastada comparándola con resultados de soluciones analíticas contenidas en manuales de factores de intensidad de tensiones [109]. Los resultados indican que con una roseta de 12 elementos los resultados de los FIT son plenamente satisfactorios, siendo prácticamente coincidentes en la estimación de $K_{I}$. En nuestro caso se han utilizado 24 elementos en la roseta.

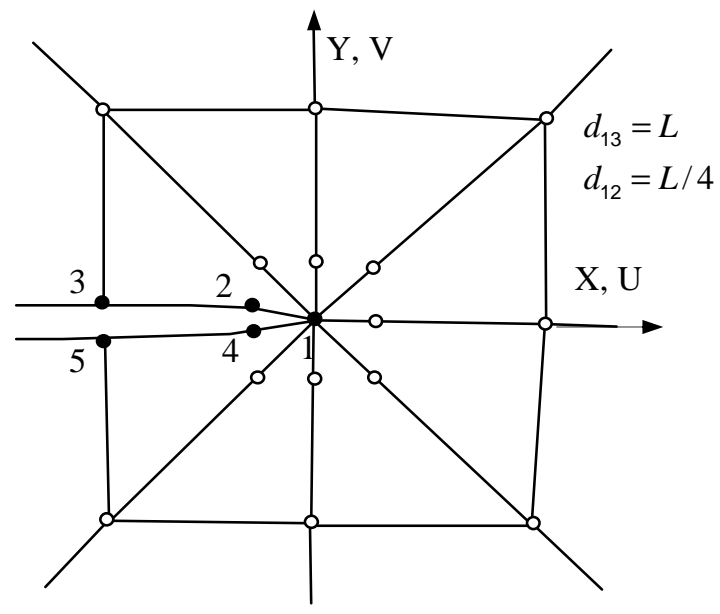

Figura 3.12. Elementos singulares en la punta de la fisura.

En la Figura 3.13 se presenta el mallado desarrollado en la probeta SPT prefisurada. Sólo se ha mallado un cuarto de la misma por presentar dos planos de simetría. Se han aplicado las adecuadas condiciones de simetría al plano de la fisura y el contorno exterior de la probeta se ha considerado con condiciones de empotramiento. El punzón ha sido modelado como un sólido elástico lineal. Para el material se ha utilizado un modelo elástico, lineal e isótropo. Estas consideraciones son válidas para cualquier tamaño de probeta y profundidad de fisura analizados.

Para facilitar al usuario final la aplicación de la metodología propuesta, se han deducido una serie de expresiones, una por cada tamaño de probeta, que permiten evaluar el valor de $K_{I}$ en función del tamaño de fisura y carga aplicada. Al igual que en otras partes de la presente Tesis, se han considerado dos tamaños de probeta diferentes, una de dimensiones 20x20x1 $\mathrm{mm}$ y otra de dimensiones típicas de 10x10x0.5mm. La primera es la base para el establecimiento de la metodología presentada y la segunda por su aplicación generalizada como tamaño de probeta SPT. 

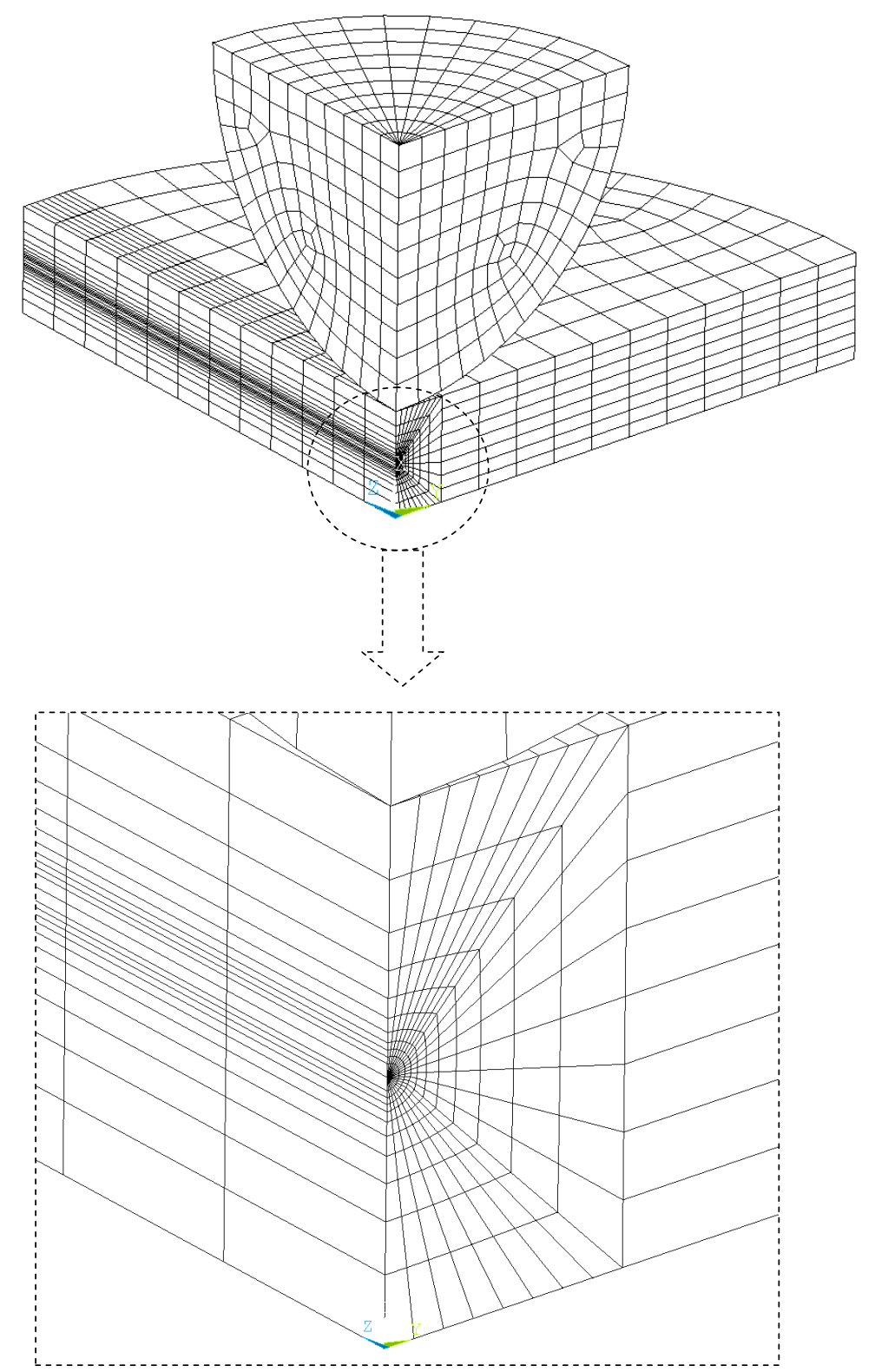

Figura 3.13. Mallado de la probeta SPT prefisurada.

Para extender el rango de aplicación de las expresiones desarrolladas se ha tenido en cuenta, además de la carga aplicada sobre la probeta $P$, la relación adimensional $a / t$, donde $a$ es la profundidad de fisura inicial y $t$ es el espesor de la probeta. Dicha relación $a / t$ se ha hecho variar entre los valores 0.1 , correspondiente a una fisura inicial de poca profundidad, y 0.7 , que representa una fisura inicial prácticamente pasante.

El valor de $K_{I}$, es evaluado en condiciones de deformación plana en el centro de la probeta, ya que es en este punto donde se produce el inicio de propagación de la fisura. Este hecho se 
comprobará experimentalmente mediante el análisis SEM de ensayos interrumpidos realizado en el Capítulo 5. Se puede observar, que es en este punto donde el valor de $K_{I}$ es máximo en todo el frente de fisura, como aparece reflejado en la Figura 3.14, que muestra el valor de $K_{I}$ en diferentes puntos del frente de fisura según su distancia al centro de la probeta para distintos valores de carga. Se puede observar como conforme va aumentando la carga $P$ la curva correspondiente tiende a apuntarse en el centro de la probeta, favoreciendo de este modo que el inicio de propagación de la fisura se produzca en dicho punto.

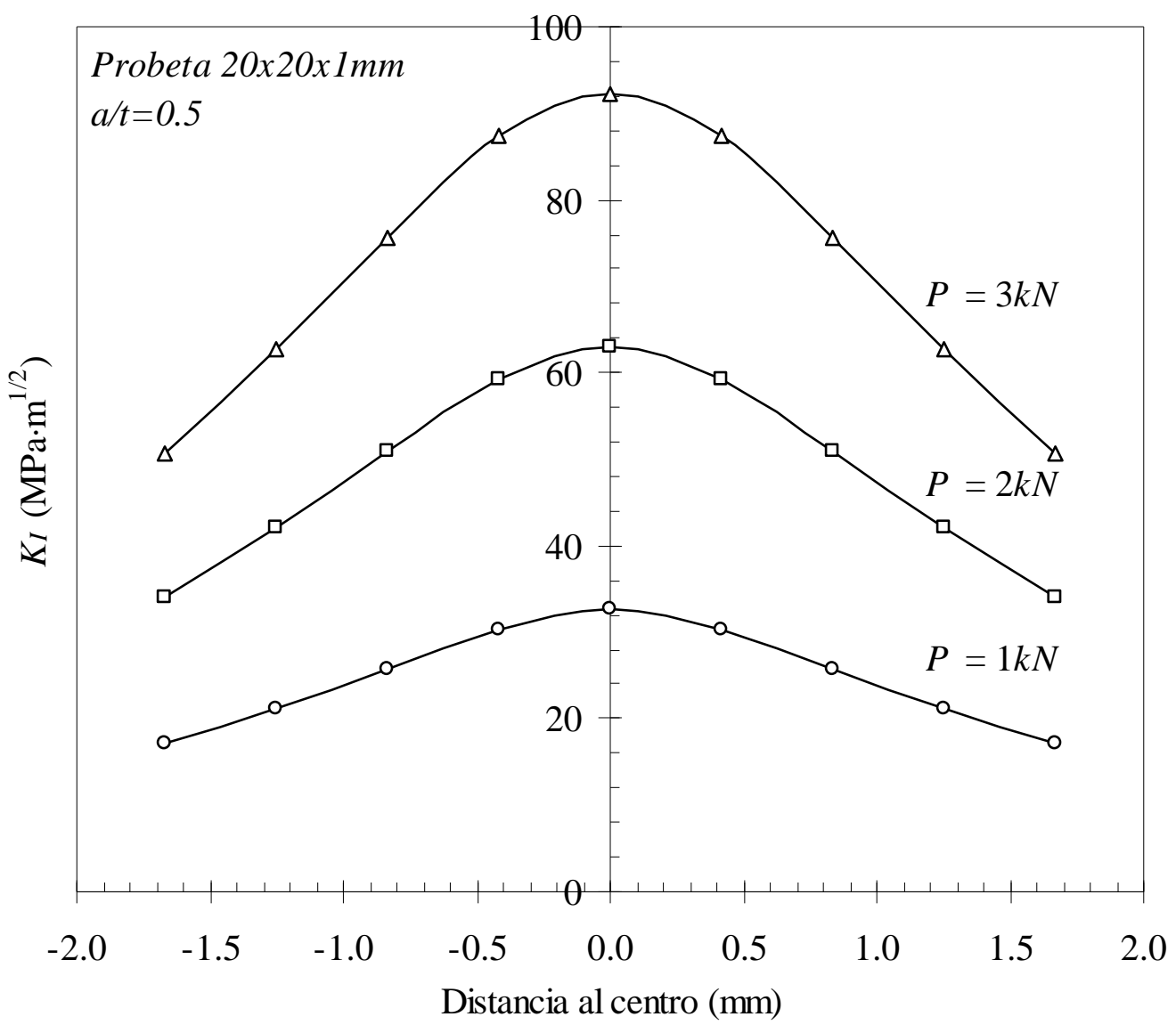

Figura 3.14. Evolución de $K_{I}$ a lo largo de la fisura, probeta $20 \times 20 \times 1 \mathrm{~mm}$, a $/ t=0.5$.

En la Tabla 3.13 y en la Tabla 3.14 se muestran los valores de $K_{I}$ obtenidos para las probetas de dimensiones 20x20x1 $\mathrm{mm}$ y 10x10x0.5 mm respectivamente, en función de la carga aplicada y de la relación $a / t$. Dichos resultados se han ajustado con una serie de curvas potenciales del tipo $K_{I}=k_{1} \cdot P^{n_{1}}$, agrupando los resultados según la relación $a / t$, donde $k_{1}$ y $n_{1}$ son las constantes que definen la curva. Cada una de las curvas ajustadas se puede observar en la 
Figura 3.15 y en la Figura 3.16 correspondientes a las probetas de 20x20x1mm y 10x10x0.5mm respectivamente. El eje de ordenadas correspondiente a $K_{I}$ se encuentra en escala logarítmica, para poder apreciar mejor la tendencia de los resultados obtenidos.

Una vez ajustados los resultados con las curvas potenciales, se ha determinado la variación que sufren las constantes $k_{1}$ y $n_{1}$ de dichas curvas potenciales en función de la relación $a / t$ y del tamaño de probeta utilizado. Dichas variaciones de $k_{1}$ y $n_{1}$ se pueden ver en la Figura 3.17 y en la Figura 3.18 respectivamente. La variación de $k_{1}$ se ajusta adecuadamente con una expresión polinómica de segundo grado, mientras que la variación de $n_{1}$ se ha realizado con una ecuación tipo neperiana inversa de segundo orden obteniendo un buen ajuste.

Finalmente, con las variaciones de $k_{1}$ y $n_{1}$ definidas matemáticamente en función de la relación $a / t$, se han deducido las expresiones (3.17) y (3.18) que permiten evaluar $K_{I}$ en función de la carga aplicada para las probetas de 20x20x1mm y 10x10x0.5mm respectivamente, estableciendo la condición de validez $0.1 \leq a / t \leq 0.7$.

$$
\begin{aligned}
& K_{I 20 \times 20 \times 1}=\left[-138.81 \cdot\left(\frac{a}{t}\right)^{2}+96.81 \cdot\left(\frac{a}{t}\right)+18.9\right] \cdot P^{0.9399-\frac{0.0461}{\ln \left(\frac{a}{t}\right)}-\frac{0.0324}{\left[\ln \left(\frac{a}{t}\right)\right]^{2}}} \\
& K_{I 10 x 10 x 0.5}=\left[-403.35 \cdot\left(\frac{a}{t}\right)^{2}+270.6 \cdot\left(\frac{a}{t}\right)+48.94\right] \cdot P^{0.9605-\frac{0.0121}{\ln \left(\frac{a}{t}\right)-\frac{0.0235}{\left[\ln \left(\frac{a}{t}\right)\right]^{2}}}}
\end{aligned}
$$

Por otro lado, la Figura 3.19 y la Figura 3.20 muestran la evolución de $K_{I}$ en función de la relación $a / t$ para distintos niveles de carga para cada uno de los tamaños de probeta utilizados. Se puede comprobar como de manera general el máximo de todas las curvas se alcanza para valores de $a / t$ entre 0.3 y 0.4 . Este hecho resulta realmente interesante, ya que se podría determinar el valor óptimo de profundidad de fisura para el cuál se alcanza el valor máximo de $K_{I}$. Dicho valor óptimo, se tendrá en cuenta más adelante a la hora de realizar las fisuras iniciales en las probetas, con las que se llevarán a cabo los ensayos SPT. Los valores teóricos de dichas fisuras iniciales se han elegido de tal manera que haya valores antes, después y en el óptimo, resultando de esta manera los valores teóricos de $a / t$ de $0.2,0.3,0.4$ y 0.5 . 
Tabla 3.13. Valores de $K_{I}$ en función de la carga aplicada y de la relación a / $t$, probeta $20 \times 20 \times 1 \mathrm{~mm}$.

\begin{tabular}{|c|c|c|c|c|c|c|c|c|c|}
\hline $\begin{array}{c}K_{I} \\
\left(M P a \cdot m^{1 / 2}\right)\end{array}$ & \multicolumn{10}{|c|}{$a / t$} \\
\hline$P(k N)$ & $\mathbf{0 . 1}$ & $\mathbf{0 . 2}$ & $\mathbf{0 . 3}$ & $\mathbf{0 . 4}$ & $\mathbf{0 . 4 5}$ & $\mathbf{0 . 5}$ & $\mathbf{0 . 5 5}$ & $\mathbf{0 . 6}$ & $\mathbf{0 . 7}$ \\
\hline $\mathbf{0 . 5}$ & 13.97 & 17.03 & 18.22 & 18.30 & 17.51 & 16.82 & 15.81 & 14.45 & 10.45 \\
\hline $\mathbf{1}$ & 26.99 & 32.92 & 35.21 & 35.16 & 34.28 & 32.75 & 30.52 & 27.50 & 18.51 \\
\hline $\mathbf{2}$ & 52.65 & 64.34 & 68.76 & 68.38 & 66.38 & 63.03 & 58.17 & 51.64 & 32.51 \\
\hline $\mathbf{3}$ & 77.45 & 94.82 & 101.33 & 100.55 & 97.37 & 92.13 & 84.60 & 74.54 & 45.60 \\
\hline $\mathbf{4}$ & 101.68 & 124.56 & 132.97 & 131.54 & 127.05 & 119.78 & 109.46 & 95.80 & 57.16 \\
\hline $\mathbf{5}$ & 125.13 & 153.41 & 163.57 & 161.33 & 155.49 & 146.15 & 133.03 & 115.81 & 67.76 \\
\hline $\mathbf{6}$ & 149.58 & 183.47 & 195.68 & 192.96 & 186.87 & 175.51 & 159.50 & 138.41 & 79.43 \\
\hline $\mathbf{7}$ & 173.27 & 212.63 & 226.72 & 223.32 & 216.32 & 202.88 & 183.99 & 159.15 & 90.06 \\
\hline
\end{tabular}

Tabla 3.14. Valores de $K_{I}$ en función de la carga aplicada y de la relación a/t, probeta $10 \times 10 \times 0.5 \mathrm{~mm}$.

\begin{tabular}{|c|c|c|c|c|c|c|c|c|c|}
\hline $\begin{array}{c}K_{I} \\
\left(M P a \cdot m^{1 / 2}\right)\end{array}$ & \multicolumn{90}{|c|}{$a / t$} \\
\hline$P(k N)$ & $\mathbf{0 . 1}$ & $\mathbf{0 . 2}$ & $\mathbf{0 . 3}$ & $\mathbf{0 . 4}$ & $\mathbf{0 . 4 5}$ & $\mathbf{0 . 5}$ & $\mathbf{0 . 5 5}$ & $\mathbf{0 . 6}$ & $\mathbf{0 . 7}$ \\
\hline $\mathbf{0 . 1 2 5}$ & 9.66 & 11.78 & 12.62 & 12.65 & 12.38 & 11.89 & 11.18 & 10.21 & 7.39 \\
\hline $\mathbf{0 . 2 5}$ & 19.08 & 23.28 & 24.90 & 24.87 & 24.24 & 23.16 & 21.58 & 19.44 & 13.09 \\
\hline $\mathbf{0 . 5}$ & 37.23 & 45.50 & 48.62 & 48.35 & 46.94 & 44.57 & 41.14 & 36.51 & 22.99 \\
\hline $\mathbf{0 . 7 5}$ & 54.76 & 67.05 & 71.65 & 71.10 & 68.85 & 65.14 & 59.82 & 52.71 & 32.25 \\
\hline $\mathbf{1}$ & 71.90 & 88.08 & 94.02 & 93.01 & 89.84 & 84.70 & 77.40 & 67.74 & 40.42 \\
\hline $\mathbf{1 . 2 5}$ & 88.48 & 108.48 & 115.66 & 114.08 & 109.95 & 103.34 & 94.07 & 81.89 & 47.92 \\
\hline $\mathbf{1 . 5}$ & 105.58 & 128.30 & 136.77 & 134.62 & 129.54 & 121.45 & 110.18 & 95.50 & 55.00 \\
\hline $\mathbf{1 . 7 5}$ & 122.45 & 147.71 & 157.45 & 154.74 & 148.66 & 139.15 & 125.97 & 108.89 & 62.23 \\
\hline $\mathbf{2}$ & 139.23 & 170.13 & 177.63 & 174.42 & 167.45 & 156.59 & 141.59 & 122.21 & 69.57 \\
\hline
\end{tabular}



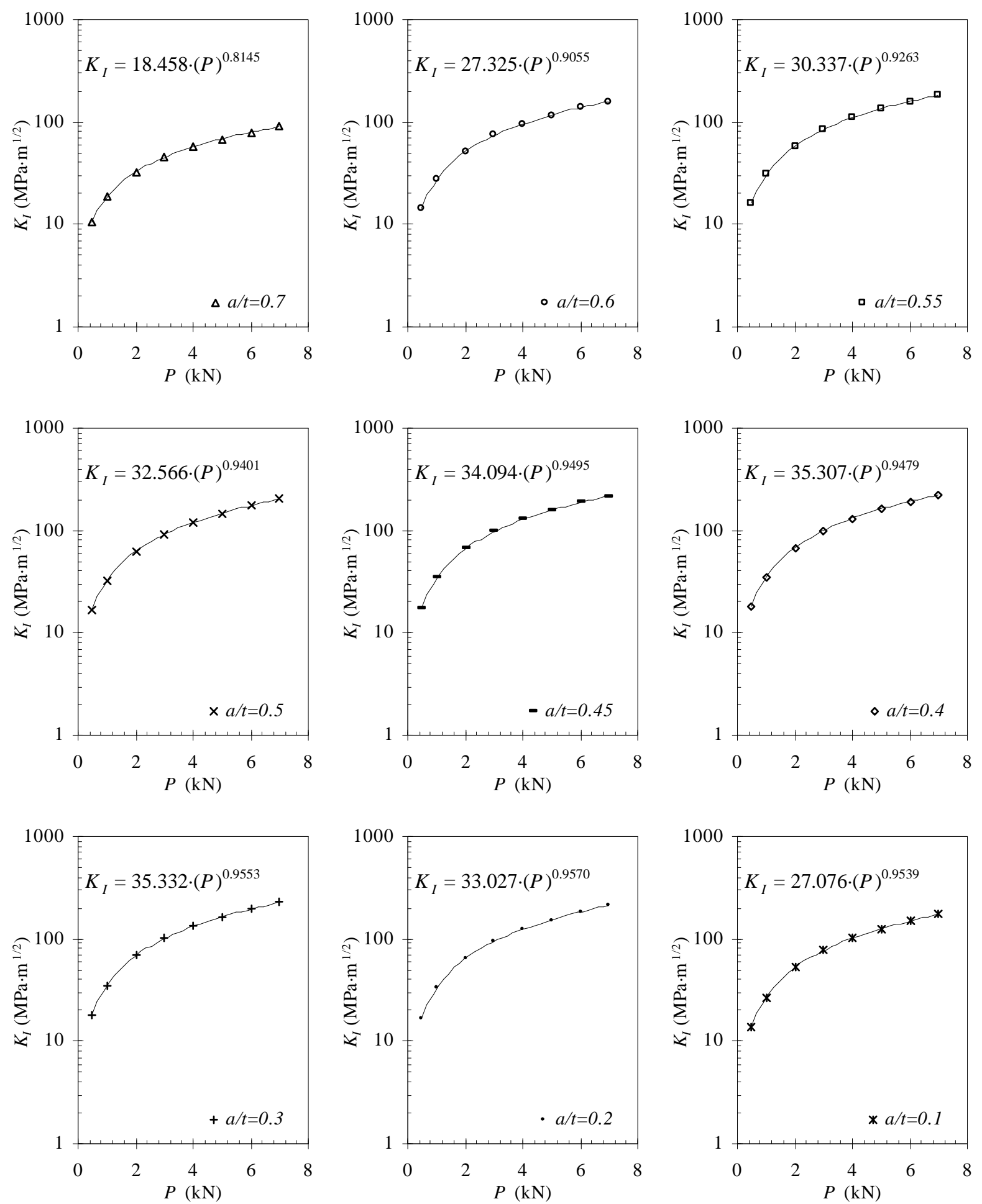

Figura 3.15. Curvas potenciales de $K_{I}$ en función de la carga aplicada y de la relación a / $t$, probeta $20 \times 20 \times 1 \mathrm{~mm}$. 

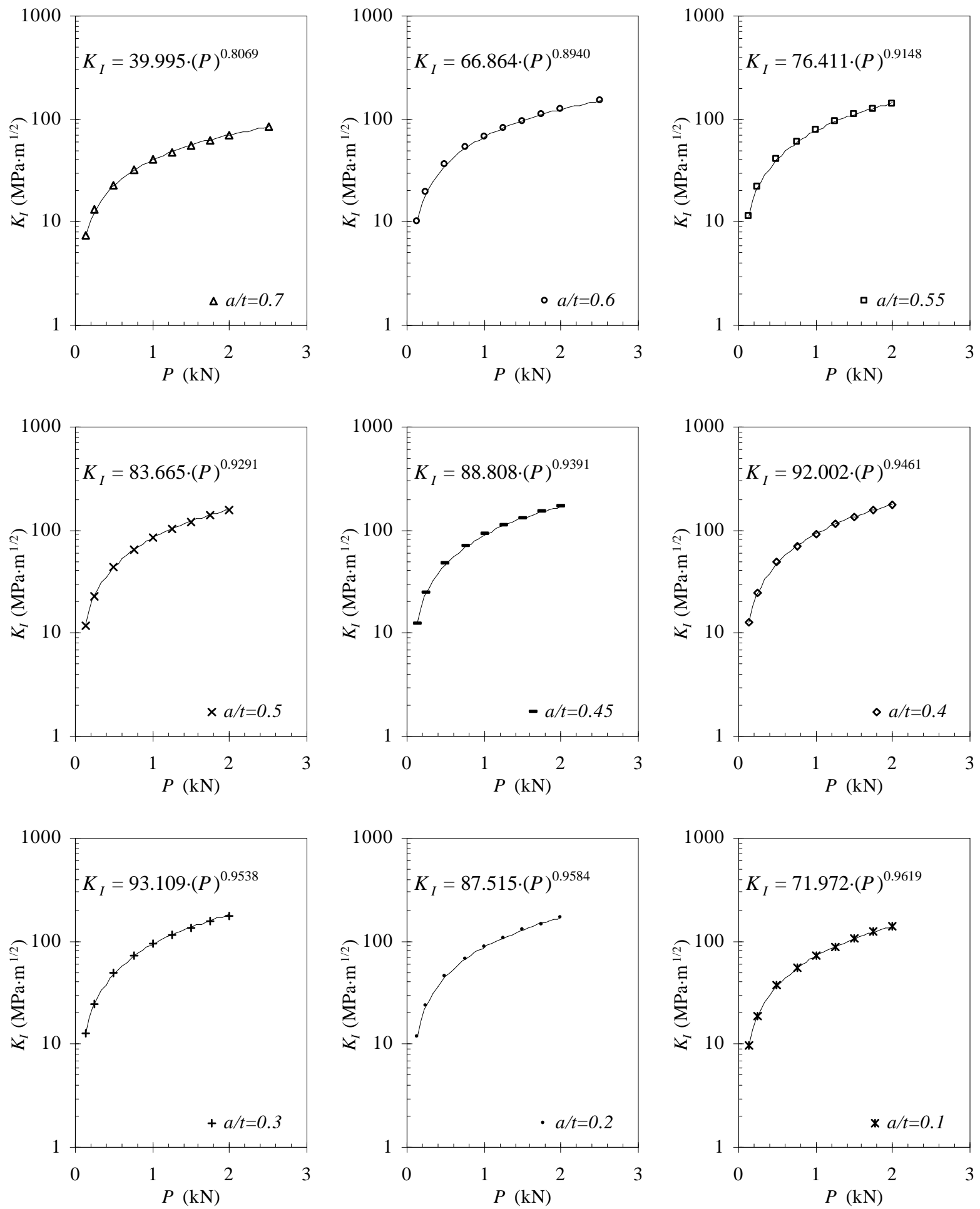

Figura 3.16. Curvas potenciales de $K_{I}$ en función de la carga aplicada y de la relación a/t , probeta $10 \times 10 \times 0.5 \mathrm{~mm}$. 


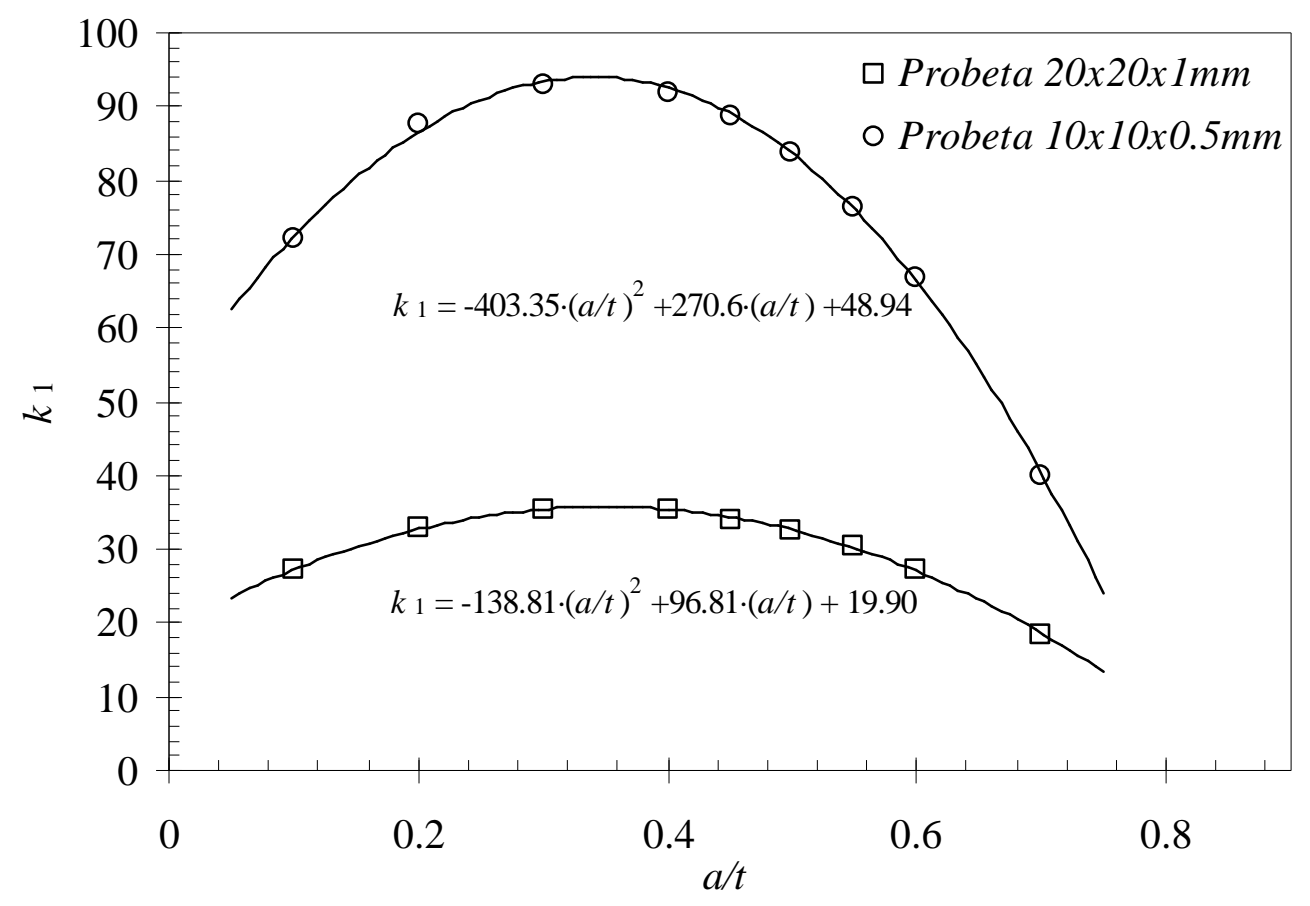

Figura 3.17. Variación de $k_{1}$ en función de la relación a $t$ y del tamaño de probeta utilizado.

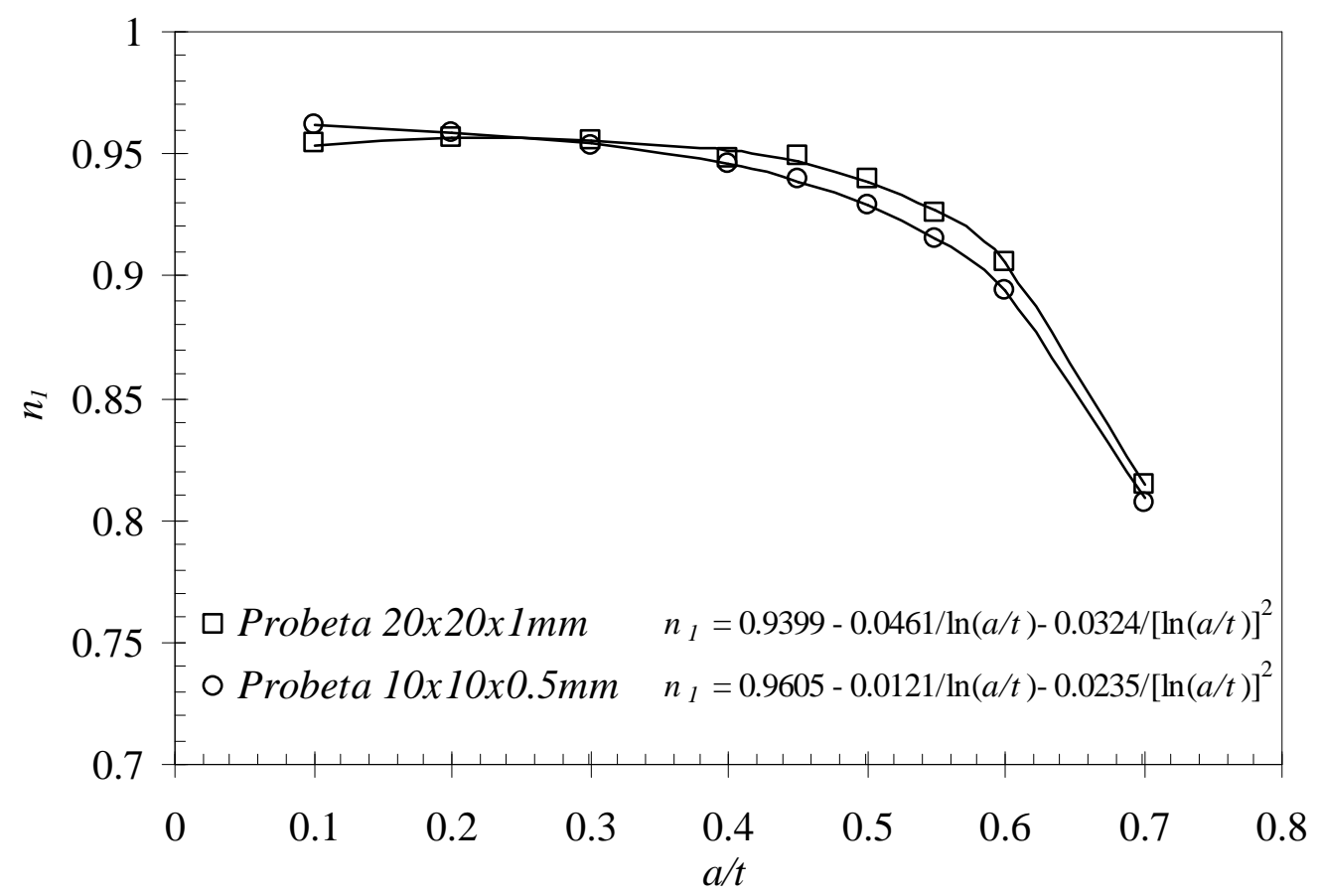

Figura 3.18. Variación de $n_{1}$ en función de la relación al $t$ y del tamaño de probeta utilizado. 


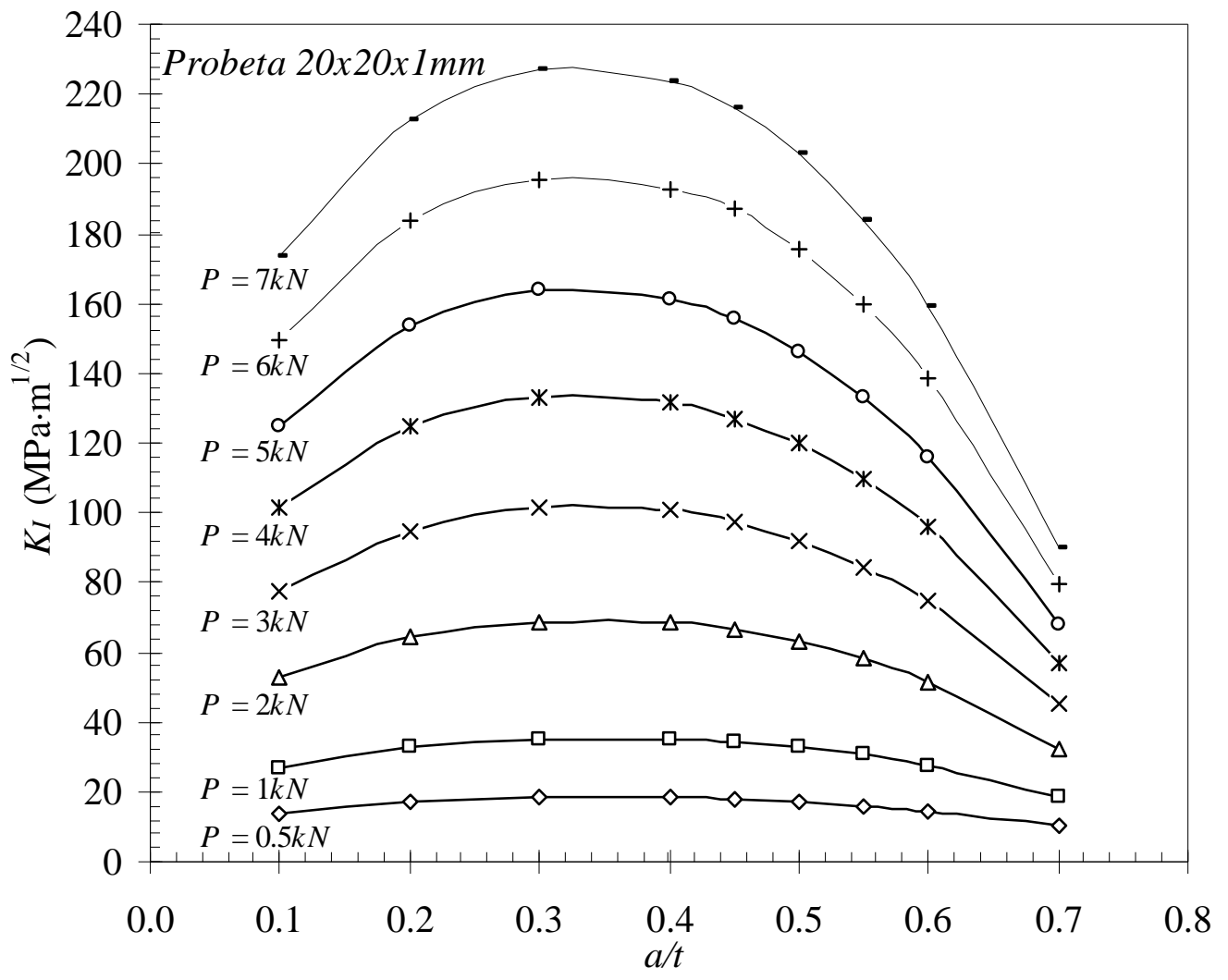

Figura 3.19. Evolución de $K_{I}$ en función de la relación a/ $t$, probeta 20x20xlmm.

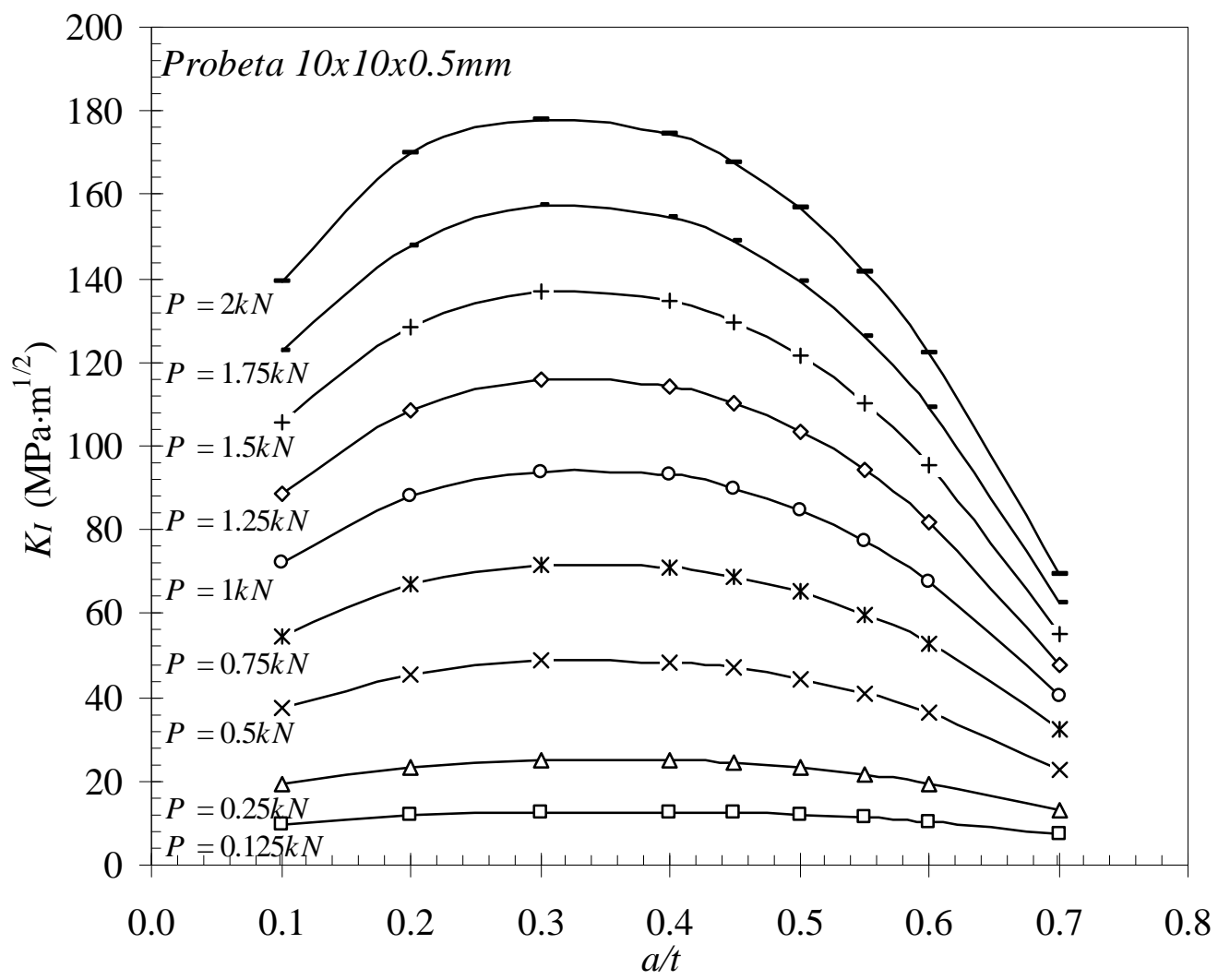

Figura 3.20. Evolución de $K_{I}$ en función de la relación a/ $t$, probeta $10 \times 10 \times 0.5 \mathrm{~mm}$. 


\subsection{DETERMINACIÓN DE $K_{\text {mat }}$}

Una vez conocidos los valores de $K_{r}$ y $K_{I}$ en el punto de inicio de propagación de fisura en la probeta es posible calcular el valor de $K_{m a t}$ de forma directa a partir de la expresión $K_{m a t}=K_{I} / K_{r}$. Con el objetivo de aplicar la metodología presentada, ésta se ha implementado sobre un acero, caracterizado en el capítulo siguiente. Los valores obtenidos se compararán con los resultados obtenidos a partir de los ensayos Charpy y de fractura realizados para su caracterización, y las recomendaciones para este material a fractura proporcionadas por el código ASME $\left(K_{m a t}=133 \mathrm{MPa} \cdot \mathrm{m}^{1 / 2}\right)$ [82], verificando de esta manera la bondad de la estimación que proporciona el método expuesto y el rango de incertidumbre para la medida de $K_{\text {mat }}$.

\subsection{CONSIDERACIONES FINALES}

Uno de los aspectos más interesantes de la presente Tesis es la utilización conjunta del SPT sobre probetas prefisuradas, del diagrama FAD, y de la simulación numérica, como se puede observar en la Figura 3.3 que representa el esquema de la metodología desarrollada. Para la realización de los ensayos SPT se han propuesto probetas prefisuradas de dimensiones 20x20 mm y espesor de $1 \mathrm{~mm}$, con el objetivo de poder analizar con mayor precisión el efecto de diferentes profundidades de fisura en los resultados obtenidos.

La generación de la fisura inicial en las probetas SPT, previa al ensayo, se ha realizado en el Instituto Tecnológico de Aragón (ITA) empleando una técnica de microfisuración por láser. Tras una calibración inicial del láser, este es aplicado sobre la superficie de la probeta para generar, una fisura longitudinal no pasante desde el centro de un lado de la probeta hasta el centro del lado opuesto. La calidad de la fisura realizada ha sido posteriormente analizada a través de técnicas de microscopía óptica de barrido.

En cuanto a las simulaciones numéricas llevadas a cabo para el desarrollo de la metodología presentada, se ha supuesto que la curva tensión-deformación del material, se puede ajustar según una ecuación tipo Ramberg Osgood. Para aceros cuya ley de comportamiento se ajuste a otro tipo de ecuación diferente, la metodología desarrollada seguiría siendo válida, 
procediendo de manera análoga en todos y cada uno de los pasos que la conforman, donde la única diferencia existente serían los parámetros de los que depende la ley del material.

En todas las simulaciones numéricas se han tenido en cuenta los dos planos de simetría existentes, posibilitando de esta forma el modelado de tan solo un cuarto de probeta, disminuyendo así el coste computacional y favoreciendo la evaluación de la carga de colapso plástico $P_{U}$ en la sección neta central del plano de fisura de la probeta. Este criterio de evaluación es el comúnmente utilizado y aconsejado por la bibliografía [82] para determinar el valor de $P_{U}$. Tanto el punzón como las matrices superior e inferior se han modelado como superficies rígidas, ya que se ha considerado que durante el ensayo la deformación que sufren estos elementos es prácticamente despreciable frente a las elevadas deformaciones que se producen en la probeta simulada.

La determinación de $P_{U}$ en probetas SPT prefisuradas se ha efectuado a través de la técnica de superficie de respuesta basada en el diseño de experimentos. Ha quedado fundamentada claramente la necesidad de fijar ciertos parámetros de los que depende $P_{U}$ y de variar los restantes en el rango de valores habituales de los aceros, ya que intentar obtener una superficie de respuesta que englobe la variación de todos y cada uno de los parámetros es completamente inviable. El modelo utilizado para obtener esta superficie de respuesta ha sido un modelo polinomial de segundo orden, el cuál es el modelo típico que se usa en presencia de tres parámetros variables. El primer diseño de experimentos recogido en este apartado, se ha llevado a cabo variando los parámetros $E, \sigma_{0.2}$ y $n$, manteniendo todos los demás constantes. La superficie de respuesta determinada, para cada tamaño de probeta, ajusta adecuadamente los valores de $P_{U}$.

Ha quedado reflejada, la gran influencia que tiene en el modelo el parámetro $\sigma_{0.2}$ y la falta de significación de $E$. Este último hecho, permite fijar dicho parámetro al valor medio del rango de variación de los aceros (200000MPa), pudiendo así realizar un nuevo diseño de experimentos con tres variables, en el que se ha incluido la relación $a / t$ como parámetro variable. Obteniendo de nuevo una función, para cada tamaño de probeta, que define la superficie de respuesta de $P_{U}$. Al igual que en el caso anterior el parámetro $\sigma_{0.2}$ es el más significativo, seguido de la relación $a / t$. 
Continuando con esta pequeña recapitulación de la metodología desarrollada, tal y como se ha comentado anteriormente, una vez conocido el valor de $L_{r}$ y antes de determinar el valor de $K_{r}$, es necesario representar el diagrama FAD, según la opción que se desee. Se ha visto como la Opción 3 de FAD es la única que lleva asociada una simulación numérica con la que calcular los parámetros $J_{a p p}$ y $J_{e}$, imprescindibles para aplicar la expresión que define dicha opción.

Por otro lado, la simulación numérica también es necesaria para evaluar el factor de intensidad de tensiones $K_{I}$. Se ha desarrollado una función, para cada una de las dos dimensiones de probeta SPT utilizadas, que permite estimar el valor de $K_{I}$. Dichas funciones están expresadas en función de la carga aplicada en la probeta y de la relación $a / t$, estableciendo para este último parámetro la condición de validez $0.1 \leq a / t \leq 0.7$. Durante el desarrollo de dichas funciones, se ha representado la evolución de $K_{I}$ en función de la relación $a / t$ para distintos niveles de carga, observando que el máximo de todas las curvas representadas se alcanza entre los valores 0.3 y 0.4 de la relación $a / t$. Lo cuál quiere decir, que es en este tramo de valores donde se alcanza el valor óptimo de profundidad de fisura, para obtener el valor máximo de $K_{I}$ en la probeta SPT utilizada, para una determinada carga aplicada. 


\section{CAPÍTULO 4}

\section{CARACTERIZACIÓN DEL MATERIAL}

\subsection{INTRODUCCIÓN}

En este capítulo se recoge la caracterización del material utilizado para la aplicación de la metodología desarrollada en el Capítulo 3 de la presente Tesis. El material elegido para dicha aplicación ha sido el acero 15-5 PH-Condición 1025, el cuál es un acero inoxidable martensítico endurecido por precipitación, utilizado en componentes de altas presiones.

Sus principales características son: alto límite elástico, buena resistencia a la corrosión, buenas propiedades mecánicas a temperaturas superiores a $316^{\circ} \mathrm{C}$ y buena tenacidad tanto en la dirección longitudinal como en la transversal. Presenta un comportamiento cercano a un material elástico-perfectamente plástico, lo cuál puede resultar, a priori, interesante para la aplicabilidad del procedimiento desarrollado a materiales con elevado grado de ductilidad.

Para la completa caracterización de dicho acero se han llevado a cabo una serie de ensayos normalizados, como son el ensayo de tracción uniaxial, el ensayo Charpy y el ensayo de tenacidad a fractura con probetas compactas CT. Con los que se determinan las principales propiedades elastoplásticas y de fractura del material, las cuáles se utilizarán en los siguientes apartados.

En el presente capítulo se presentan los resultados obtenidos en dichos ensayos normalizados, haciendo especial hincapié en los resultados que posteriormente se utilizarán para la consecución de los objetivos de la presente Tesis. 


\subsection{MATERIAL}

Como se ha comentado previamente, el material utilizado en la presente Tesis es un acero inoxidable martensítico endurecido por precipitación conocido como acero $15.5 \mathrm{PH}$ (condición 1025). Está también caracterizado por la temperatura del tratamiento térmico al que se somete, en nuestro caso condición 1025 (calentamiento de cuatro horas a una temperatura de $1025^{\circ} \mathrm{F} \pm 15^{\circ} \mathrm{F}$ y enfriamiento al aire).

Según los datos proporcionados por el fabricante del acero, el proceso de fundición se ha realizado acorde con el código ASME Sección VIII División 3 Parte KM Artículo KM-4, el proceso de conformado se ha efectuado conforme al código ASME Sección II SA 705MApartado 5, y el tratamiento térmico acorde con el código ASME Sección II SA-7.5M Tipo SM-12 y Sección VIII División 3 Parte KM. La composición química y las propiedades mecánicas típicas para este acero quedan recogidas en la Tabla 4.1 y en la Tabla 4.2 respectivamente. Además en la Tabla 4.1 aparece la composión química medida para el material recepcionado, comprobando que los valores se encuentran dentro del margen establecido.

Tabla 4.1. Composición química del acero 15-5 PH, típica y medida.

\begin{tabular}{|c|c|c|c|c|c|c|c|c|c|}
\hline Acero 15-5 PH & $\mathrm{C}$ & $\mathrm{Mn}$ & $\mathrm{Si}$ & $\mathrm{Ni}$ & $\mathrm{Cr}$ & $\mathrm{Cu}$ & $\mathrm{Nb}$ & $\mathrm{P}$ & $\mathrm{S}$ \\
\hline Valores típicos & $<0.07$ & $<1.0$ & $<1.0$ & $3.5 / 5.5$ & $14.0 / 15.5$ & $2.5 / 4.5$ & $0.15 / 0.45$ & $<0.04$ & $<0.03$ \\
\hline Recepcionado & 0.043 & 0.810 & 0.280 & 5.000 & 14.660 & 3.060 & 0.380 & 0.017 & 0.001 \\
\hline
\end{tabular}

Tabla 4.2. Propiedades mecánicas del acero 15-5 PH suministradas por el fabricante.

\begin{tabular}{|c|c|c|}
\hline Propiedades Mecánicas & Valor & Comentarios \\
\hline Dureza, Rockwell C & $39(40)$ & Transversal (Longitudinal) \\
\hline Tensión última de tracción $(M P a)$ & 1200 & Longitudinal \\
\hline Tensión última de tracción $(M P a)$ & 1204 & Transversal \\
\hline Límite elástico $(M P a)$ & 1176 & $0.2 \%$ YS Longitudinal \\
\hline Elongación $(\%)$ & 12.20 & En 2 pulgadas, Longitudinal \\
\hline Módulo elástico en tensión $(M P a)$ & 196000 & \\
\hline
\end{tabular}




\subsection{COMPORTAMIENTO EN TRACCIÓN UNIAXIAL}

La caracterización del comportamiento del material en tracción se ha efectuado mediante el ensayo de probetas convencionales siguiendo la norma ASTM E 8M [110]. La Figura 4.1 muestra las dimensiones normalizadas de la probeta utilizada. El tratamiento de la curva tensión-deformación ha permitido conocer los parámetros referentes al módulo de elasticidad del material $E$, el límite elástico convencional $\sigma_{0.2}$, la tensión última $\sigma_{u t s}$ y el exponente de endurecimiento del material $n$. Además se ha determinado la curva tensión-deformación real, y extrapolada para grandes deformaciones, para su utilización en las simulaciones numéricas que sean necesarias posteriormente.

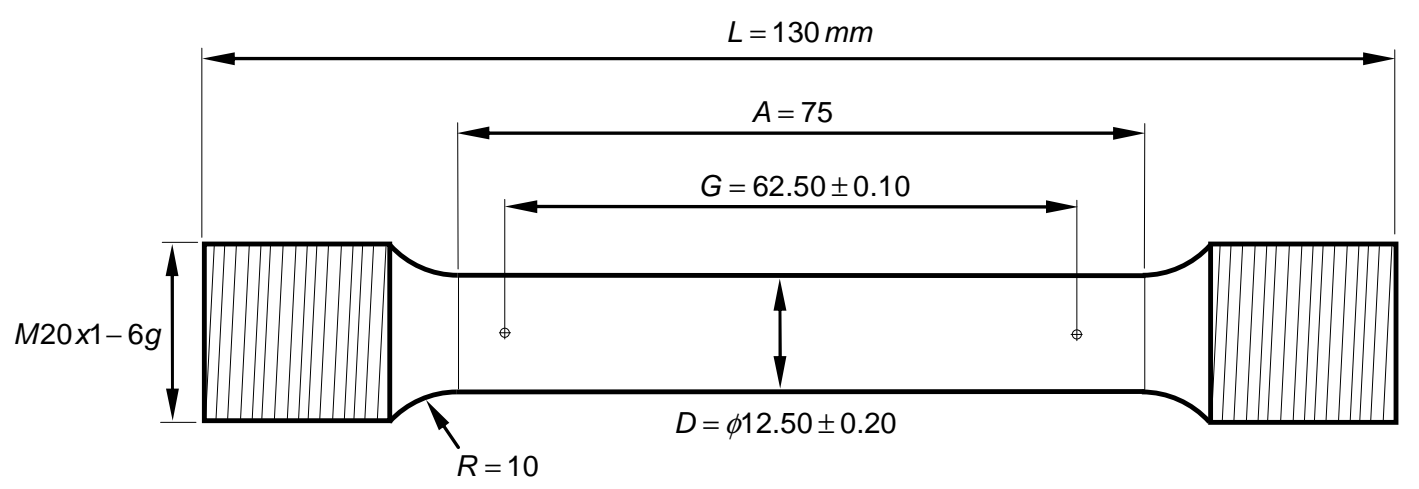

Figura 4.1. Dimensiones normalizadas de probeta para el ensayo de tracción [110].

Las probetas han sido obtenidas a partir de dos anillos de acero 15-5 PH de diámetro interior $275 \mathrm{~mm}$ y diámetro exterior de $385 \mathrm{~mm}$, siendo el espesor del primero de $30 \mathrm{~mm}$ y el del segundo de $160 \mathrm{~mm}$. Para analizar el comportamiento del material en las diferentes direcciones se han mecanizado probetas en las direcciones circunferencial (A2B1) y transversal (C1B1) del anillo, no obteniendo diferencias significativas en el comportamiento mecánico, lo que indica el elevado grado de isotropía del material.

El ensayo se ha realizado con una máquina de ensayo MTS 810 modelo 318.25, con un rango de fuerza hasta $250 \mathrm{kN}$. En todos los ensayos se ha utilizado una cámara climática MTS modelo 651.06E-03, la cuál permite ensayar en un rango de temperaturas desde $-129^{\circ} \mathrm{C}$ hasta $316^{\circ} \mathrm{C}$, y un extensómetro MTS 632.24F-50. Para garantizar el buen alineamiento de la carga durante el ensayo se ha utilizado un mecanismo de alineación de rótula convenientemente lubricado. 
Las curvas tensión-deformación ingenieril obtenidas en el ensayo de tracción para las probetas A2B1 y C1B1 se muestran en la Figura 4.2. A partir de estas curvas es posible obtener las curvas tensión-deformación verdadera (ver Figura 4.3) y los parámetros característicos del ensayo de tracción $\left(E, \sigma_{0.2}, \sigma_{u t s}\right.$ y $n$ ) recogidos en la Tabla 4.3 respectivamente.

Como podemos observar, los valores de los parámetros son muy similares a los típicos para este tipo de materiales, que aparecen recogidos en el apartado anterior. Así mismo se aprecia el elevado grado de isotropía del material, y la escasa influencia de la orientación en el comportamiento en tracción.

Tabla 4.3. Parámetros elasto-plásticos de las probetas A2B1 y C1B1.

\begin{tabular}{|c|c|c|c|c|c|}
\cline { 3 - 6 } \multicolumn{2}{c|}{} & \multicolumn{4}{c|}{ Parámetros elasto-plásticos } \\
\hline Probeta & Dirección & $E(M P a)$ & $\sigma_{0.2}(M P a)$ & $\sigma_{\text {uts }}(M P a)$ & $n$ \\
\hline A2B1 & Circunferencial & 191426 & 1100 & 1131 & 38.8 \\
\hline C1B1 & Transversal & 190018 & 1107 & 1138 & 39 \\
\hline
\end{tabular}

El valor del parámetro $E$ es el correspondiente a la pendiente del tramo elástico de la curva tensión-deformación ingenieril, el valor de $\sigma_{0.2}$ se ha determinado trazando una recta paralela al tramo elástico de la curva anterior que pase por un valor de deformación del $0.2 \%, \sigma_{u t s}$ es el máximo valor de la tensión ingenieril a partir del cuál comienza la estricción en la probeta y el valor de $n$ se ha calculado, una vez conocidos los parámetros anteriores, ajustando la curva tensión-deformación verdadera a una curva Ramberg Osgood.

Para realizar este ajuste se han descartado los valores próximos a la tensión máxima verdadera, aproximadamente a partir de un valor de deformación del 10\%, debido a la aparición de daño local en la probeta de ensayo. La Figura 4.4 muestra dicho ajuste para la probeta A2B1, se puede observar como para grandes deformaciones superiores al $10 \%$ las dos curvas dejan de ajustar correctamente, ya que en la curva Ramberg Osgood no se tiene en cuenta el daño que se produce en la probeta de ensayo. 


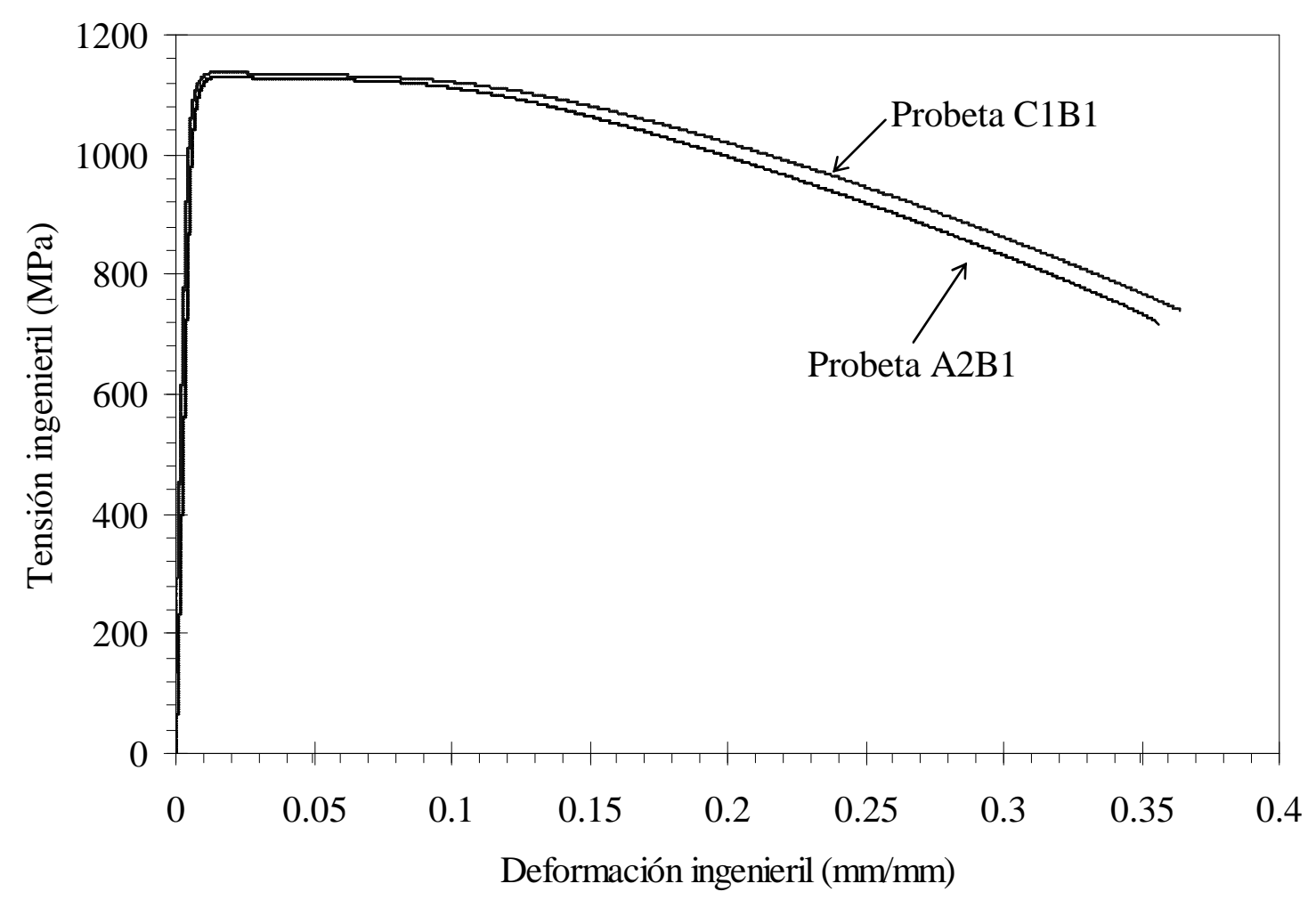

Figura 4.2. Curvas tensión-deformación ingenieril del acero $15-5 \mathrm{PH}$, temperatura $20^{\circ} \mathrm{C}$.

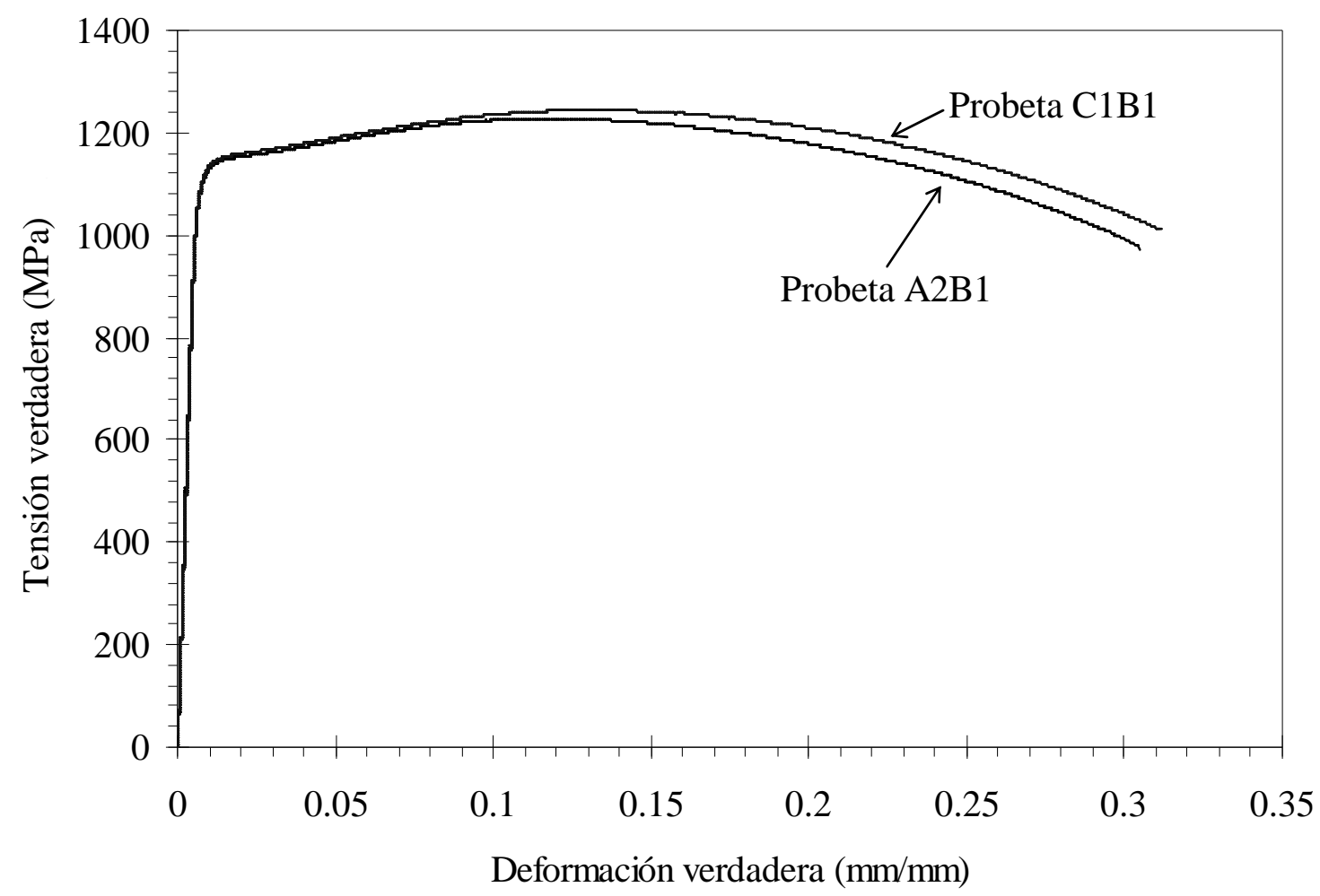

Figura 4.3. Curvas tensión-deformación verdadera del acero $15-5 \mathrm{PH}$, temperatura $20^{\circ} \mathrm{C}$. 


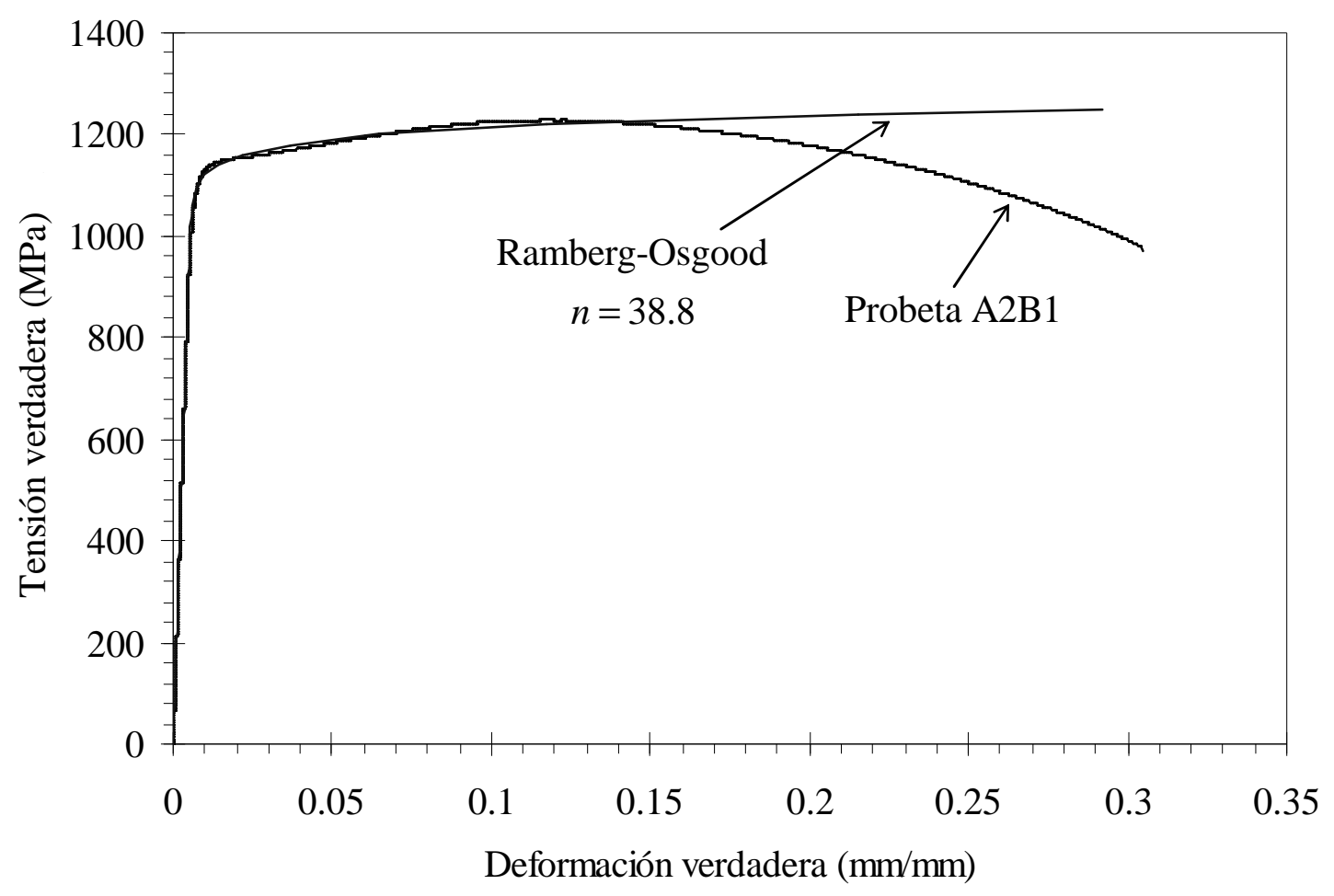

Figura 4.4. Curva tensión-deformación verdadera del acero 15-5 PH ajustada según una ecuación tipo Ramberg-Osgood [97] definida por la expresión (3.5).

Se ha llevado a cabo un análisis fractográfico mediante microscopía electrónica de barrido (SEM) para analizar el modo característico de rotura de este material. Tal y como se puede observar en la Figura 4.5, la superficie de fractura exhibe el aspecto típico de la fractura dúctil, con nucleación y coalescencia de microhuecos como causantes de la rotura final. Dentro de algunos microhuecos se han encontrado partículas de alúmina y carburos, como se puede ver en la Figura 4.6, aunque se ha observado que la presencia de estas partículas es poco habitual en la superficie de fractura.

Las probetas SPT prefisuradas de 20x20x1 $\mathrm{mm}$, utilizadas en la presente Tesis, han sido obtenidas de una barra correspondiente a la dirección circunferencial, por lo que los valores de los parámetros elastoplásticos correspondientes a la probeta A2B1 recogidos en la Tabla 4.3 serán los que se van a tomar de referencia cuando sea necesario su uso en la presente Tesis, así como la utilización de la curva tensión-deformación verdadera de dicha probeta, extrapolada sin daño para grandes deformaciones, para las simulaciones numéricas que así lo requieran. 

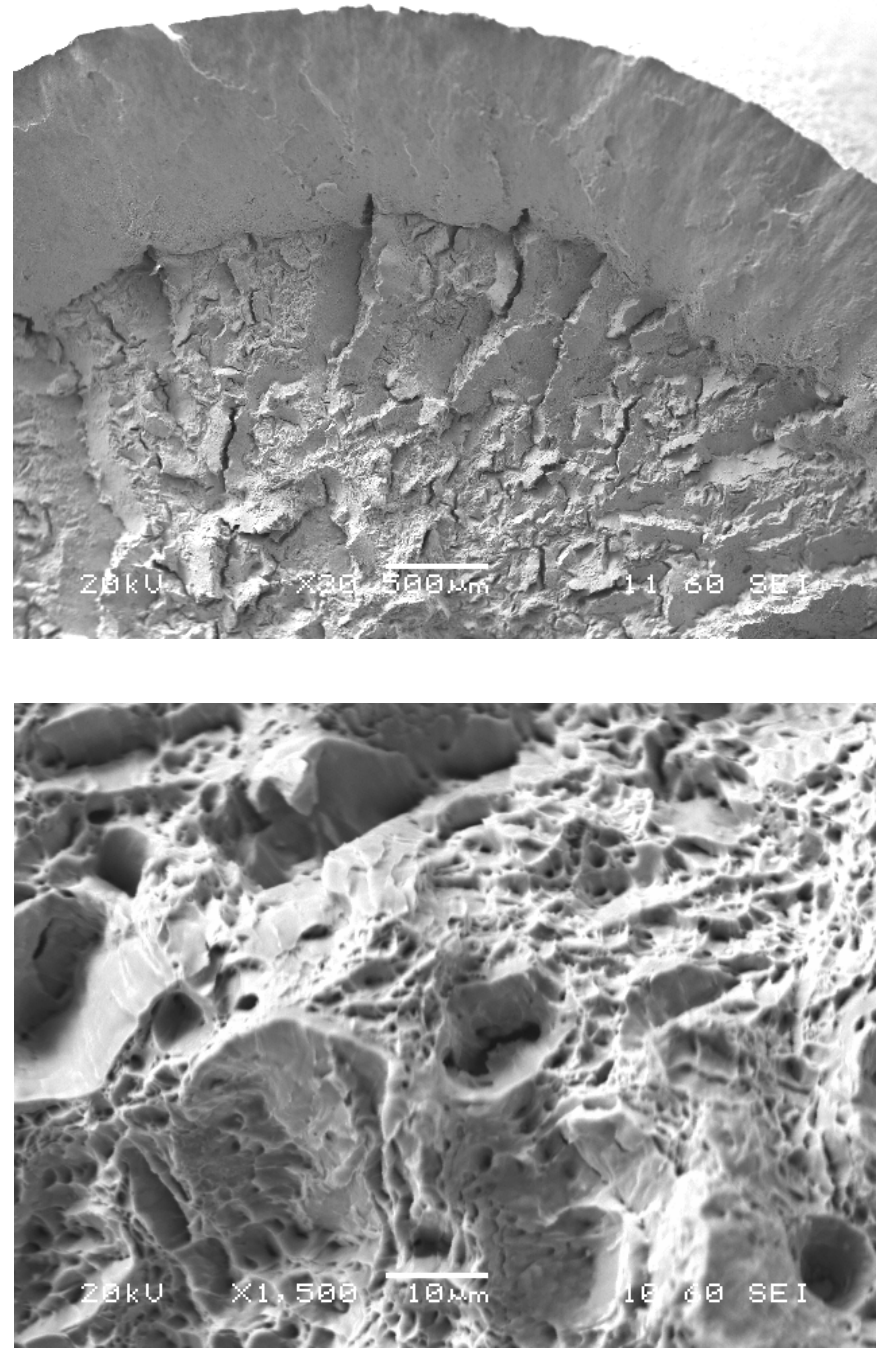

Figura 4.5. Superficie de fractura de la probeta C1B1.

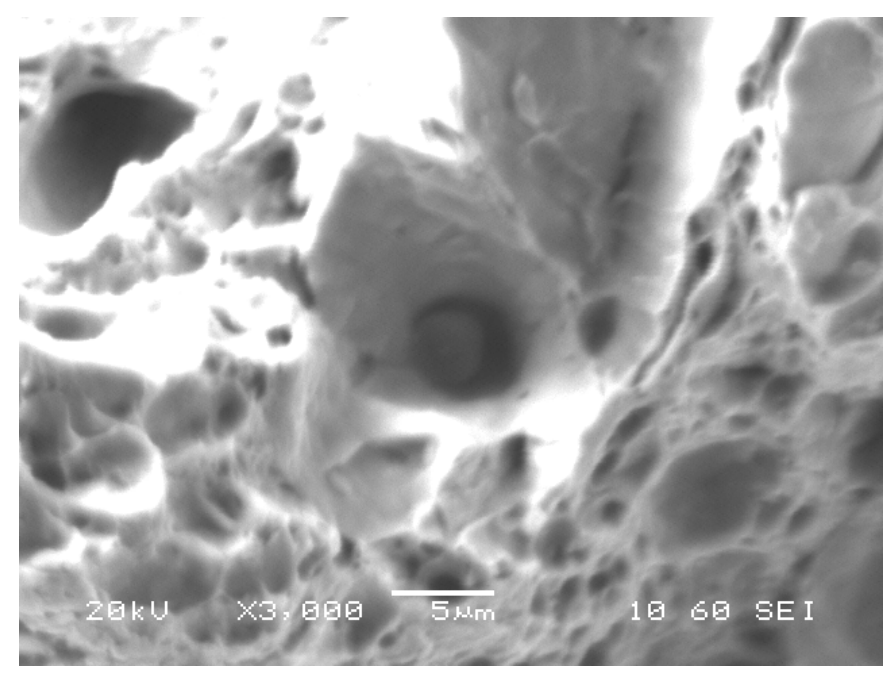

Figura 4.6. Inclusión en la superficie de fractura de la probeta ClB1. 


\subsection{ESTIMACIÓN DE PROPIEDADES ELASTOPLÁSTICAS}

Tal y como ha quedado patente en varias ocasiones en la presente Tesis, uno de los pilares en los que se fundamenta la aplicación de la metodología desarrollada, es la determinación de los parámetros elastoplásticos del material utilizado. En la mayoría de los casos en los que se desee aplicar esta metodología, puesto que esa es una de sus motivaciones, no se va a disponer de material suficiente para llevar a cabo ensayos normalizados que permitan conocer estos parámetros. En consecuencia, es necesario utilizar el propio ensayo SPT como base para la determinación de las propiedades elastoplásticas del material, para poder dar solución a este inconveniente.

A continuación se presenta un procedimiento para estimar estos parámetros. Dicho procedimiento está basado en el estudio que realizaron Mao y Takahashi [4], en la mecánica de medios continuos y en la simulación numérica.

\subsubsection{Estimación de los parámetros $\sigma_{y}, \sigma_{u t s}, n$ y $\sigma_{0.2}$ a partir del SPT}

Mao y Takahashi [4] fueron los primeros en realizar un estudio empírico sobre diversos aceros con el objetivo de relacionar los parámetros $\sigma_{y}$ y $\sigma_{u t s}$ con las cargas $P_{y}$ y $P_{\max }$ de la curva carga-desplazamiento del SPT respectivamente, a partir de probetas circulares de $3 \mathrm{~mm}$ de diámetro y espesor $0.25 \mathrm{~mm}$ y probetas cuadradas de $10 \times 10 \mathrm{~mm}$ de espesores 0.3 y $0.5 \mathrm{~mm}$. Los resultados obtenidos por estos investigadores ya han sido comentados anteriormente, así como las expresiones analíticas que determinaron para la estimación de $\sigma_{y}$ y $\sigma_{u t s}$, definidas por las expresiones (2.4) y (2.5), que recordando eran:

$$
\begin{aligned}
& \sigma_{y}=360 \cdot P_{y} / t_{0}^{2} \\
& \sigma_{u t s}=130 \cdot P_{\max } / t_{0}^{2}-320
\end{aligned}
$$

En los casos que así lo requieran serán estas expresiones las utilizadas para estimar $\sigma_{y}$ y $\sigma_{u t s}$, a partir de los datos de $P_{y}$ y $P_{\max }$ extraídos de la curva carga-desplazamiento obtenida en un ensayo SPT con probeta convencional (sin fisura inicial). 
Las expresiones empíricas anteriores, únicamente proporcionan valores estimados de $\sigma_{y} \mathrm{y}$ $\sigma_{u t s}$. En este trabajo pretende obtenerse, no solo el límite elástico y la tensión de rotura, sino también el exponente de endurecimiento $n$. Para ello, supongamos que el comportamiento del material puede ajustarse mediante una ecuación tipo Ramberg Osgood, definida anteriormente en la expresión (3.2) como:

$$
\varepsilon=\varepsilon_{e}+\varepsilon_{p}=\frac{\sigma}{E}+K_{1} \cdot\left(\frac{\sigma}{E}\right)^{n}
$$

Existen infinitas curvas función de $n$ que pueden unir los puntos correspondientes al mismo límite elástico $\sigma_{y}$ y tensión última $\sigma_{u t s}$, tal y como aparece reflejado en la Figura 4.7. Ese efecto también aparece reflejado en las curvas carga-desplazamiento del SPT, como se puede apreciar en la misma figura, donde la diferencia debida al valor de $n$ comienza a ser notable en las regiones iniciales de la curva.
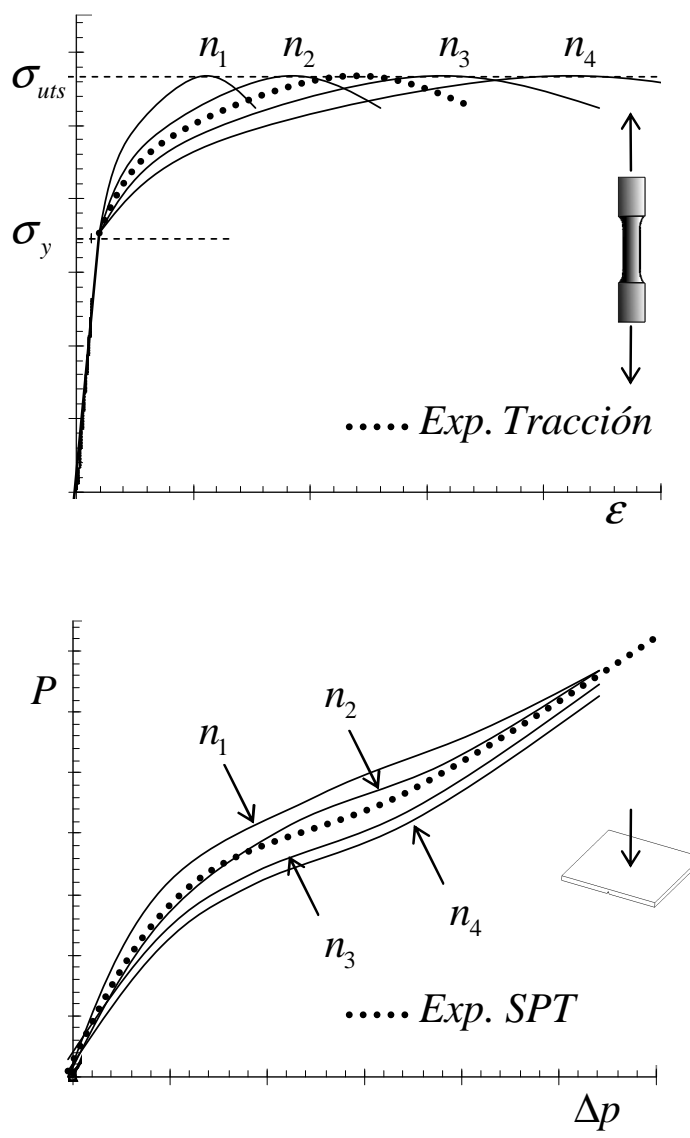

Figura 4.7. Efecto de la variación del valor de $n$ en el ensayo de tracción y en el SPT. 
Para estimar el valor de $n$, en el presente trabajo se propone simular el SPT con un determinado número de curvas de comportamiento del material en las que $n$ sea variable, para identificar con cuál de ellas se logra un mejor ajuste con el ensayo experimental de SPT. Para definir dichas curvas se dispondría únicamente de los valores de $\sigma_{y}$ y $\sigma_{u t s}$, estimados en el paso anterior, los cuáles presentan una relación con los parámetros $n$ y $K_{1}$ de la ecuación Ramberg Osgood que puede determinarse a partir de la condición de inestabilidad plástica $\left(\frac{\partial \sigma}{\partial \varepsilon}=\sigma\right)$ definida por la mecánica de medios continuos, según el desarrollo matemático expuesto a continuación.

En efecto, para deformaciones cercanas a la inestabilidad (grandes deformaciones), despreciando la parte elástica, la ecuación Ramberg Osgood se puede expresar como,

$$
\varepsilon \simeq \varepsilon_{p}=K_{1} \cdot\left(\frac{\sigma}{E}\right)^{n}
$$

Despejando $\sigma$ y haciendo un cambio de variable obtenemos,

$$
\sigma=E \cdot\left(\frac{\varepsilon}{K_{1}}\right)^{1 / n}=\frac{E}{K_{1}^{1 / n}} \cdot \varepsilon^{1 / n}=C \cdot \varepsilon^{m}
$$

donde $C$ y $m$ son las constantes que definen dicho cambio de variable, quedando establecidas por las expresiones,

$$
\begin{aligned}
& C=\frac{E}{K_{1}^{1 / n}} \\
& m=\frac{1}{n}
\end{aligned}
$$

Según la teoría de la mecánica de los medios continuos, la inestabilidad plástica o condición de carga máxima de un material se alcanza cuando se satisface la siguiente condición, $\partial \sigma / \partial \varepsilon=\sigma$. En consecuencia, derivando la expresión (4.2) respecto a $\varepsilon$ se obtiene,

$$
\frac{\partial \sigma}{\partial \varepsilon}=C \cdot m \cdot \varepsilon^{m-1}
$$


igualando las ecuaciones (4.2) y (4.5), obtenemos que en el instante en el que se produce la inestabilidad $\varepsilon=m$.

Por otro lado, las relaciones existentes entre las variables reales e ingenieriles en el ensayo de tracción son:

$\varepsilon=\ln (1+e)$

$\sigma=s \cdot(1+e)$

donde $s$ y $e$ son la tensión y deformación ingenieril, y $\sigma$ y $\mathcal{E}$ son la tensión y deformación real respectivamente. En consecuencia, resultaría inmediato convertir una curva tensióndeformación ingenieril en tensión-deformación real (o verdadera), utilizando para ello las expresiones (4.6) y (4.7). En el punto de inestabilidad, en el cuál se alcanza el máximo en la curva $s-e$, la tensión alcanza el valor de la tensión ingenieril última $\sigma_{u t s}$, también denominada $s_{u}$, se puede establecer la relación (4.8) utilizando algunas de las expresiones vistas anteriormente en este apartado.

$\left.\begin{array}{l}\sigma=C \cdot \varepsilon^{m} \\ \sigma=s_{u} \cdot(1+e) \\ \varepsilon=\ln (1+e) \\ \varepsilon=m\end{array}\right\} \Rightarrow C \cdot \varepsilon^{m}=s_{u} \cdot \exp \varepsilon \Rightarrow C \cdot m^{m}=s_{u} \cdot \exp m \Rightarrow C=\frac{s_{u} \cdot \exp m}{m^{m}}$

Deshaciendo el cambio de variable efectuado anteriormente obtenemos la relación (4.9) existente entre los parámetros $K_{1}$ y $n$.

$\frac{E}{K_{1}^{1 / n}}=\frac{s_{u} \cdot \exp (1 / n)}{\left(\frac{1}{n}\right)^{1 / n}} \Rightarrow K_{1}^{1 / n}=\frac{E \cdot\left(\frac{1}{n}\right)^{1 / n}}{s_{u} \cdot \exp (1 / n)} \Rightarrow K_{1}=\frac{E^{n} \cdot\left(\frac{1}{n}\right)}{\left(s_{u} \cdot \exp (1 / n)\right)^{n}}$

Lo cuál indica que el valor de $K_{1}$ de la ecuación Ramberg Osgood puede obtenerse a partir de $n, s_{u}$ y $E$. Una vez estimados los valores de $\sigma_{y}, \sigma_{u t s}$ y $n$, puede determinarse de manera sencilla el valor del límite elástico convencional para una deformación plástica del $0.2 \%$ 
$\left(\sigma_{0.2}\right)$ directamente en la ecuación Ramberg Osgood. Otra posible forma de estimar $\sigma_{0.2}, \mathrm{y}$ con idéntico resultado, sería a partir de la representación de la curva Ramberg Osgood que define el material trazar una recta paralela al tramo elástico de la curva anterior que pase por un valor de deformación del $0.2 \%$, el punto de corte entre la curva y la recta paralela correspondería en el eje de ordenadas con el valor de $\sigma_{0.2}$. Este valor de $\sigma_{0.2}$ es una variable de entrada cuando se utiliza la superficie de respuesta determinada en el Capítulo 3 para la determinación del valor de la carga de colapso plástico $P_{U}$.

El procedimiento presentado permitirá determinar los parámetros $\sigma_{y}, \sigma_{u t s}, n$ y $\sigma_{0.2}$, que definen el comportamiento elastoplástico del material analizado, utilizando la curva cargadesplazamiento de un ensayo SPT convencional. A continuación se presenta la aplicación de este procedimiento para el acero 15-5 $\mathrm{PH}$, utilizado en las probetas SPT ensayadas en la presente Tesis.

\subsubsection{Caso práctico de la estimación de los parámetros $\sigma_{y}, \sigma_{u t s}, n$ y $\sigma_{0.2}$ a partir del SPT}

Siguiendo las indicaciones marcadas en el apartado anterior, el primer paso a ejecutar, con el objetivo de poder aplicar las expresiones analíticas desarrolladas por Mao y Takahashi [4] para la estimación de $\sigma_{y}$ y $\sigma_{u t s}$ en el acero 15-5 PH, es efectuar un ensayo SPT. Se ha utilizado una probeta convencional de $10 \times 10 \mathrm{~mm}$ y espesor $0.504 \mathrm{~mm}$, obteniendo la curva carga-desplazamiento de la Figura 4.8, en la que se ha identificado el valor de la carga máxima en el ensayo, el cuál está entorno a $2.8 \mathrm{kN}$.

Una vez determinado el valor de $P_{\max }$, quedaría por conocer el valor de $P_{y}$ para poder estimar $\sigma_{y}$ y $\sigma_{u t s}$. Para ello se ha utilizado el método propuesto por Mao y Takahashi, denominado "Método de las dos tangentes", que consiste en prolongar los tramos lineales de la curva carga-desplazamiento que se encuentran antes y después de la transición entre la Zona I y la Zona II de la curva (Figura 4.9), y determinar el punto de corte de las dos rectas. En el ejemplo presentado se obtiene un valor de carga del punzón de $0.489 \mathrm{kN}$, el cuál es considerado como el valor de $P_{y}$. 


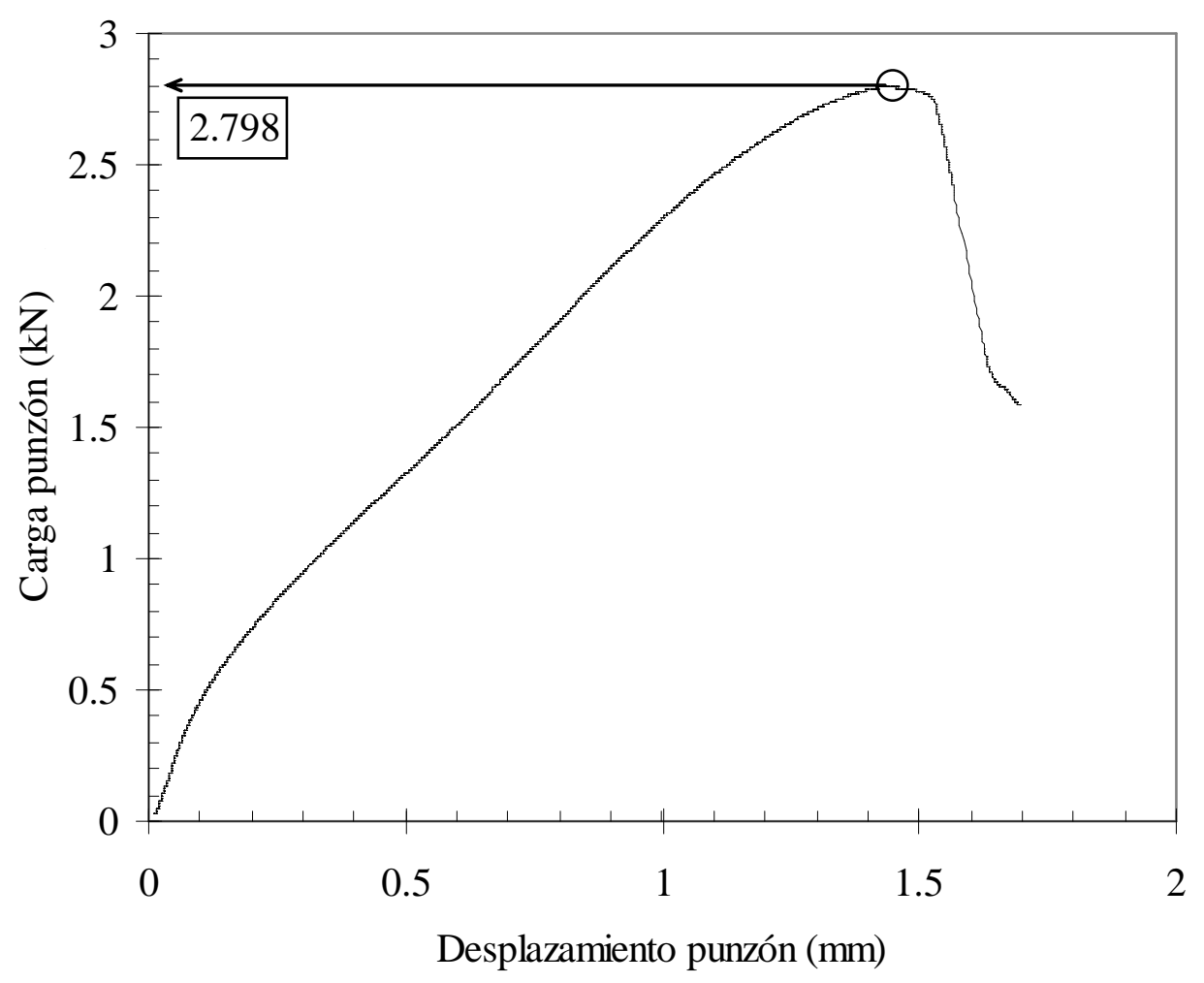

Figura 4.8. Curva carga-desplazamiento del SPT para el acero 15-5 PH.

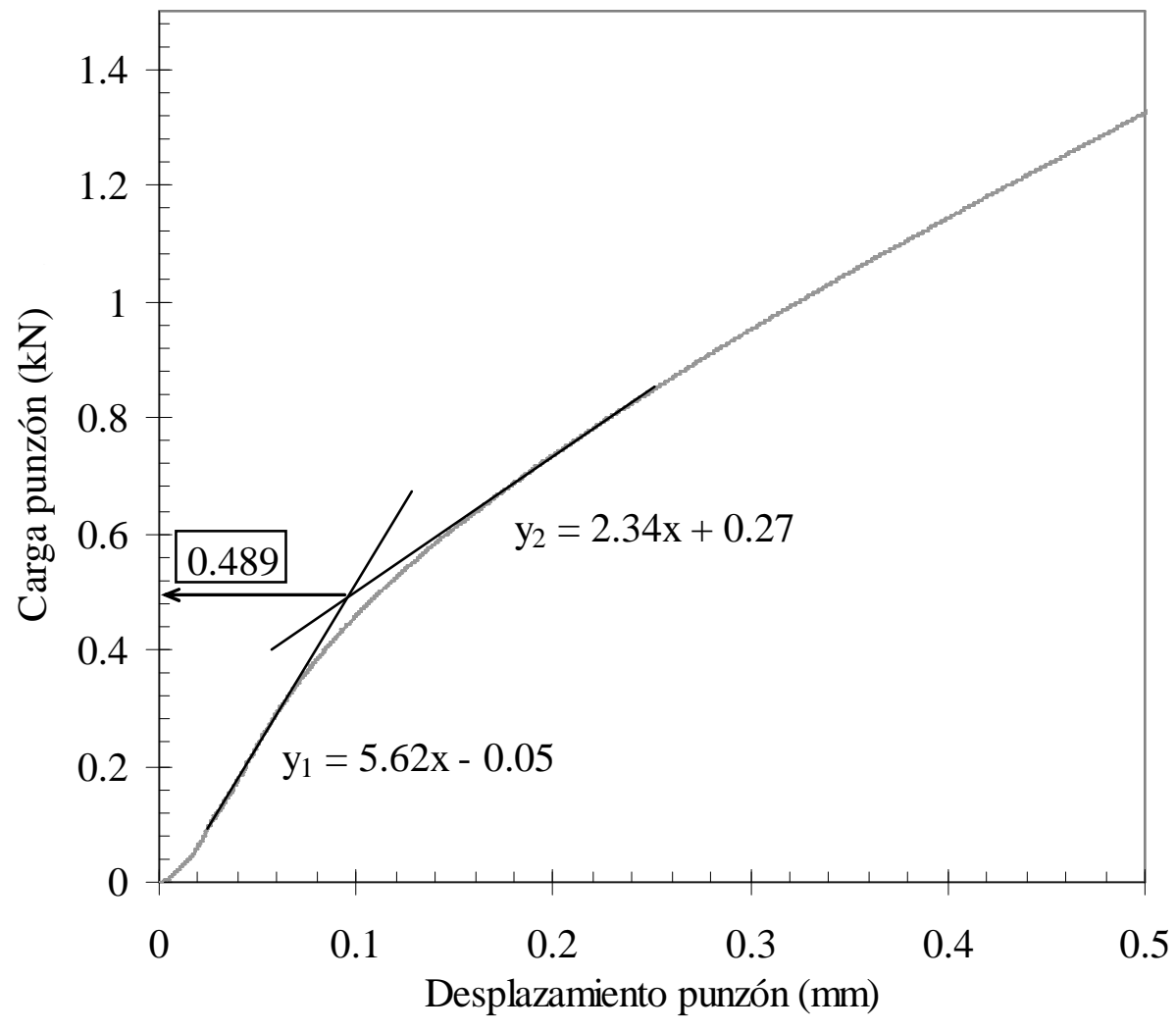

Figura 4.9. Método de las dos tangentes para la obtención de $P_{y}$. 
La Tabla 4.4 muestra la hoja Excel desarrollada para la estimación de $\sigma_{y}$ y $\sigma_{u t s}$, en la que tendríamos como variables de entrada el espesor de la probeta $t_{0}$, la pendiente $\mathrm{m}$, la ordenada en el origen $\mathrm{n}_{0}$ de las rectas determinadas en la Figura 4.9 y el valor de $P_{\max }$. Y como variables de salida, los valores de $P_{y}, \sigma_{y}$ y $\sigma_{u t s}$ del acero 15-5 PH.

Tabla 4.4. Valores estimados de $\sigma_{y}$ y $\sigma_{u t s}$ para el acero 15-5 PH.

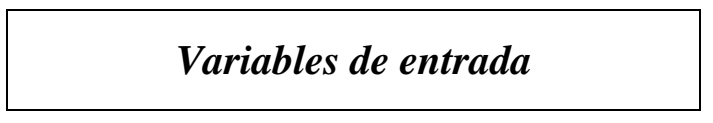

\section{Variables de salida}

\begin{tabular}{|c|c|c|}
\cline { 2 - 3 } \multicolumn{1}{c|}{} & $\mathrm{m}$ & $\mathrm{n}_{0}$ \\
\hline $\mathrm{y}_{1}$ & 5.62 & -0.05 \\
\hline $\mathrm{y}_{2}$ & 2.34 & 0.27 \\
\hline
\end{tabular}

\begin{tabular}{|c|c|}
\hline$P_{y}(k N)$ & 0.489 \\
\hline \begin{tabular}{|l|l|}
\hline$\sigma_{y}(M P a)$ & 692.9 \\
\hline
\end{tabular}
\end{tabular}

\begin{tabular}{|l|l|}
\hline Espesor $t_{0}(\mathrm{~mm})$ & 0.504 \\
\hline
\end{tabular}

$$
\begin{array}{|l|l|}
\hline \sigma_{u t s}(M P a) & 1111.9 \\
\hline
\end{array}
$$

\begin{tabular}{|l|l|}
\hline Carga máxima $P_{\max }(k N)$ & 2.798 \\
\hline
\end{tabular}

Una vez obtenidos los valores de $\sigma_{y}$ y $\sigma_{u t s}$, el siguiente paso sería la estimación del exponente de endurecimiento $n$. Siguiendo la metodología presentada en el apartado anterior supongamos que disponemos, como dato de partida para la identificación de $n$, exclusivamente la curva del ensayo SPT de la Figura 4.8. Inicialmente podemos estimar, a partir de bibliografía o de medidas de microdureza, el valor del exponente de endurecimiento aproximado de nuestro material.

En caso contrario, supongamos tres valores estándar que caracterizan los diferentes comportamientos elastoplásticos de los aceros. Los valores propuestos son 4, 12 y 30. Cuando $n=4$ estamos ante un acero con un endurecimiento por plastificación elevado, por el contrario para valores de $n$ entorno a 30 nos encontramos con un acero con un comportamiento perfectamente plástico sin apenas endurecimiento por plastificación. El valor de $n=12$ representa un comportamiento intermedio a los dos anteriores. 
Los valores de $K_{1}$ correspondientes a cada valor de $n$ se han determinado en función de la relación existente entre ellas, definida por la expresión (4.9), utilizando el valor de $\sigma_{u t s}$ determinado anteriormente y un valor representativo de $E=200000 \mathrm{MPa}$. El valor de $E$ no es significativo, como se ha puesto de manifiesto anteriormente, en la aplicación de las técnicas de superficie de respuesta, ya que una variación de éste dentro del rango de valores típicos para los aceros no influye en los resultados obtenidos para el valor de la carga de colapso plástico $P_{U}$.

En la Figura 4.10 se ven las curvas tensión-deformación de estos tres comportamientos del material, en función de $n$ para el mismo valor de $\sigma_{y}$ y $\sigma_{u t s}$ determinado anteriormente, junto con la curva real del material obtenida mediante el ensayo de tracción (desconocida a priori en este ejemplo).

Se observa que la curva que mejor ajustaría el comportamiento real del acero sería la correspondiente al valor de $n=30$, ya que en nuestro caso el acero $15-5 \mathrm{PH}$ es extremadamente elastoplástico, acercándose al comportamiento perfectamente plástico (el valor de $n$ real obtenido a partir de la curva de tracción es $n=38.8$ ). Asumamos este error como factible en un análisis real en el que no conocemos la curva tensión-deformación del material.

Discernir a partir del ensayo SPT cuál es el valor de $n$ más adecuado, sin conocer la curva de tracción a priori, resulta complicado. Sin embargo, puede estimarse un valor de $n$, y en consecuencia de $K_{1}$, realizando una batería de simulaciones numéricas variando el valor de $n$ y analizando las curvas carga-desplazamiento simuladas con la experimental del SPT. Viendo a que curva se acerca más la experimental, se puede estimar un valor de $n$ que mejore los análisis posteriores.

El tramo de curva analizado es el correspondiente a las Zonas I y II de la curva de SPT, ya que son las únicas zonas válidas para realizar la comparación debido a que a partir de la Zona III comienza la aparición de daño en la probeta experimental, el cuál no se ha tenido en cuenta en las simulaciones numéricas. En la Figura 4.11 se pueden comparar las Zonas I y II de la curva experimental de SPT frente a las curvas obtenidas en la simulación para los diferentes valores de $n$ establecidos anteriormente. 


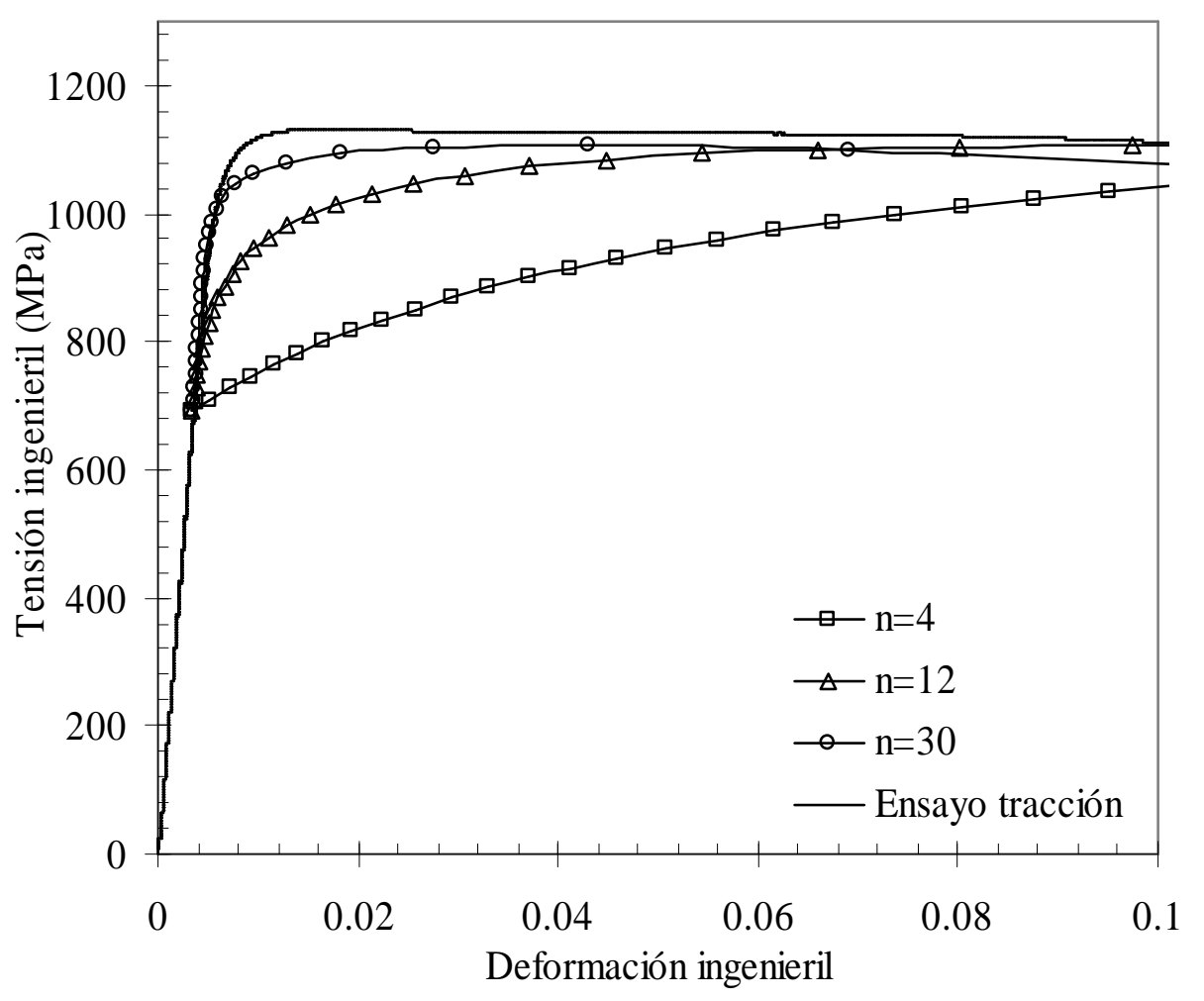

Figura 4.10. Curvas tensión-deformación ingenieril en función de $n$.

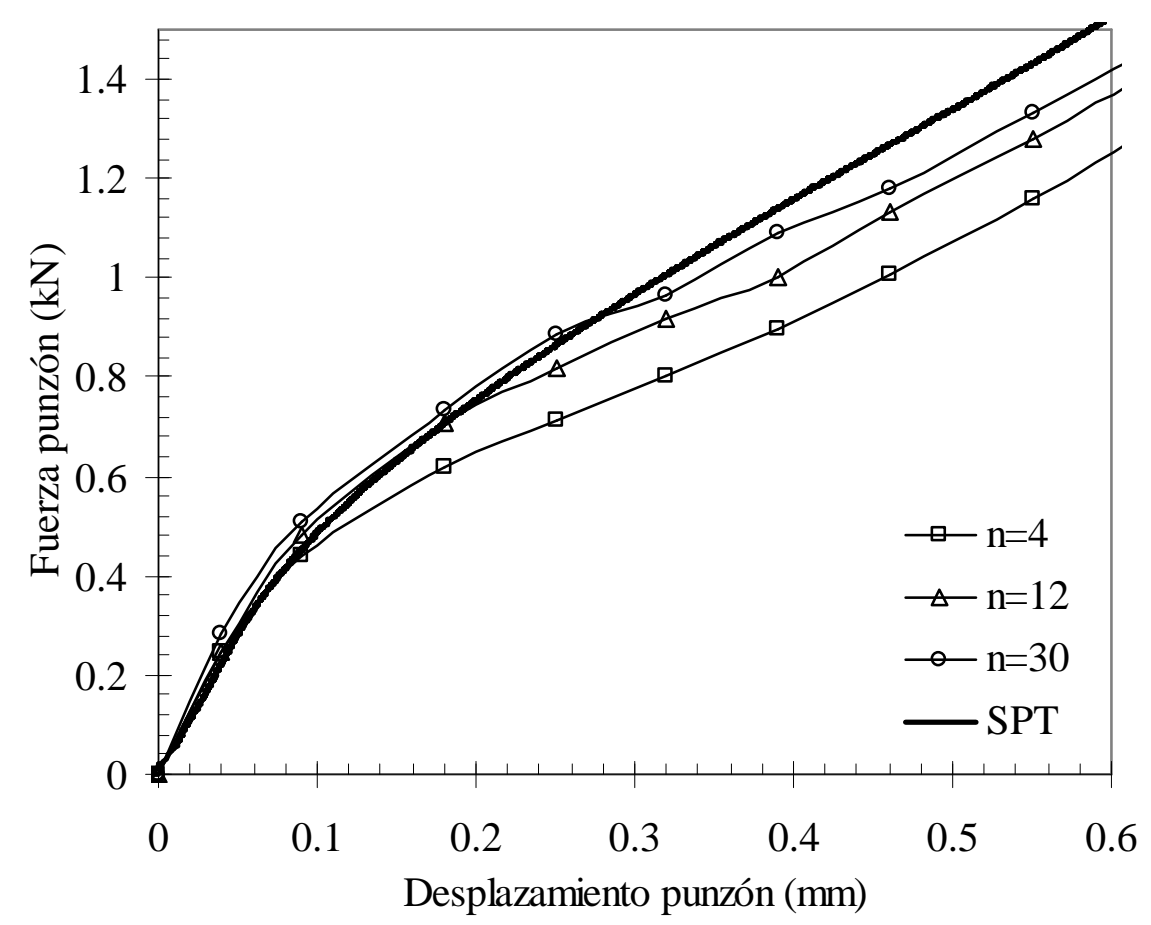

Figura 4.11. Zonas I y II de las curvas simuladas y SPT. 
Se aprecia como el ajuste de la curva experimental no es excesivamente bueno a ninguna de las curvas obtenidas a partir de la simulación numérica, siendo la que mejor ajusta la correspondiente al valor de $n=30$, por lo que sería este valor el que se utilizaría en las simulaciones posteriores que así lo requieran. Esta pequeña falta de ajuste es debida principalmente a que las expresiones analíticas desarrolladas por Mao y Takahashi [4] para la estimación de los valores de $\sigma_{y}$ y $\sigma_{u t s}$ proceden de un ajuste empírico y pese a ser válidas, presentan cierto error.

Por ejemplo, en nuestro caso el valor real de $\sigma_{u t s}$ obtenido a partir de la curva de tracción en el apartado 4.3 es de 1131MPa y el estimado con la curva de SPT es de 1111.9MPa. Esa diferencia, que puede no parecer excesiva, modifica las curvas simuladas y dificulta la obtención de $n$ a través de la comparación de la curva experimental con las simuladas de SPT.

Por último, quedaría por estimar el valor de $\sigma_{0.2}$. Esta operación sería realmente sencilla, ya que tan sólo habría que introducir en la parte plástica de la ecuación Ramberg Osgood los valores estimados para una deformación plástica del $0.2 \%$, el valor que se obtiene es $\sigma_{0.2}=1046.7 \mathrm{MPa}$.

La Tabla 4.5 recoge tanto los valores de los parámetros estimados para el acero 15-5 PH según el procedimiento desarrollado en el presente apartado, como los valores determinados en el apartado 4.3. Dichos valores serán los utilizados en los apartados del Capítulo 5 que así lo requieran.

Tabla 4.5. Parámetros elastoplásticos para el acero 15-5 PH.

\begin{tabular}{|c|c|c|}
\cline { 2 - 3 } \multicolumn{1}{c|}{} & $\begin{array}{c}\text { Parámetros elastoplásticos } \\
\text { estimados }(\text { SPT })\end{array}$ & $\begin{array}{c}\text { Parámetros elastoplásticos } \\
\text { reales (ensayo tracción) }\end{array}$ \\
\hline$E(\mathrm{MPa})$ & 200000 & 191426 \\
\hline$\sigma_{y}(\mathrm{MPa})$ & 692.9 & - \\
\hline$\sigma_{u t s}(\mathrm{MPa})$ & 1111.9 & 1131 \\
\hline$n$ & 30 & 38.8 \\
\hline$\sigma_{0.2}(\mathrm{MPa})$ & 1046.7 & 1100 \\
\hline
\end{tabular}




\subsection{ENSAYO CHARPY}

Los ensayos de tenacidad al impacto, permiten determinar la resiliencia, o capacidad de absorber energía del material ante un impacto, a diferentes temperaturas. El procedimiento del ensayo consiste en dejar caer un péndulo desde una altura determinada, ambos normalizados, de modo que incida sobre una probeta, rompiéndola y prosiguiendo su camino ascendente. Así, la energía absorbida por el material durante el impacto será la diferencia entre la energía potencial, inicial y final del péndulo, determinada esta última a partir de la altura máxima alcanzada después del impacto.

Las probetas empleadas para el ensayo de estos materiales son de flexión normalizadas, de $55 \mathrm{~mm}$ de longitud y sección cuadrada de lado $10 \mathrm{~mm}$, con entalla en $\mathrm{V}$ de $2 \mathrm{~mm}$ de profundidad. En la Figura 4.12 se presenta un esquema con la geometría de la probeta tipo A utilizada. Las probetas han sido obtenidas en la dirección circunferencial, a partir de uno de los anillos de acero 15-5 PH que se han utilizado en los ensayos de tracción, el cuál posee un diámetro interior de $275 \mathrm{~mm}$, un diámetro exterior de $385 \mathrm{~mm}$ y un espesor de $160 \mathrm{~mm}$.
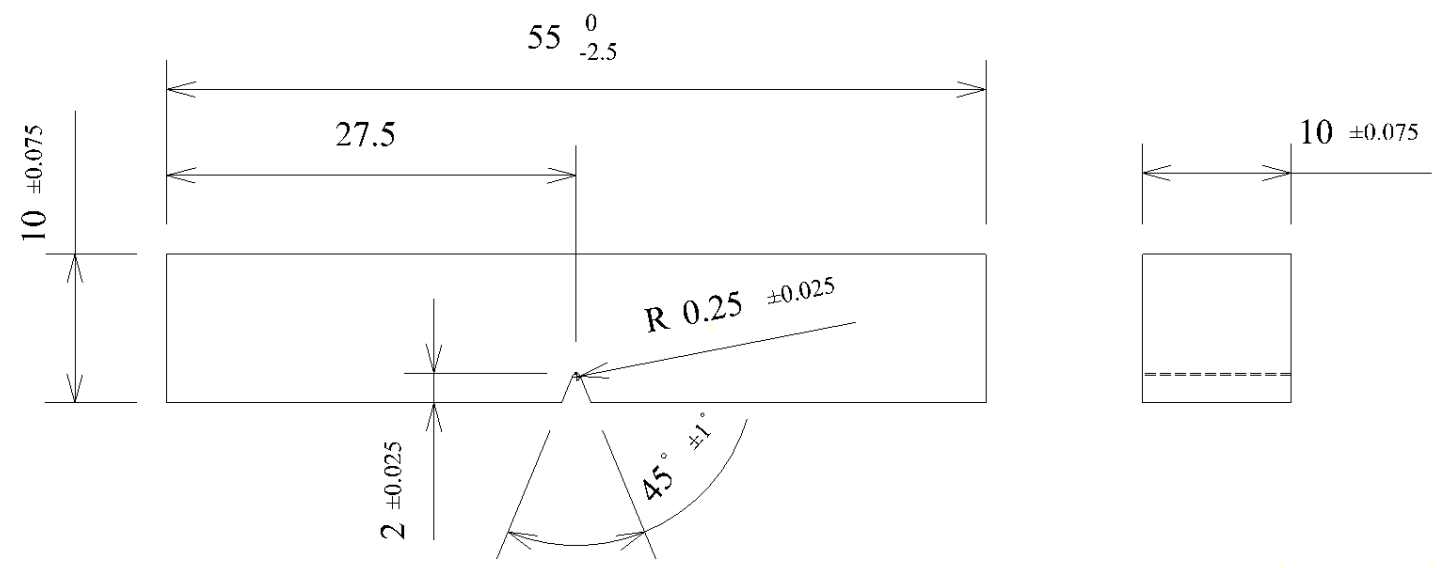

Figura 4.12. Dimensiones normalizadas de probeta tipo A para el ensayo Charpy [111].

El ensayo se ha realizado con un péndulo Charpy HOYTOM, con un rango de fuerza hasta 300J, siguiendo las recomendaciones de la norma ASTM E23 Sección 7 [111]. En todos los ensayos se ha utilizado una cámara climática Weiss Technik modelo 125B/+10IU/40DU. La calibración del péndulo, la medida de la expansión lateral de las probetas ensayadas y la obtención del porcentaje de fractura dúctil se han realizado conforme a la norma ASTM E23. Con el objetivo de conocer el comportamiento a rotura en la zona de transición dúctil-frágil 
del acero 15-5 $\mathrm{PH}$, se han realizado ensayos Charpy en un rango de temperaturas desde $-80^{\circ} \mathrm{C}$ hasta $120^{\circ} \mathrm{C}$, los resultados obtenidos se pueden observar en la Figura 4.14. Los valores anteriores se han ajustado a través de una ecuación del tipo tangente hiperbólica definida por la expresión (4.10), según el código ASME API 579 [82],

$$
C V N=A_{C V}+B_{C V} \cdot \tanh \left[\frac{\left(T-D_{C V}\right)}{C_{C V}}\right]
$$

donde $A_{C V}, B_{C V}, C_{C V}$ y $D_{C V}$ son constantes que se determinan a partir de los resultados obtenidos, y cuyo significado físico aparece recogido en el esquema de la Figura 4.13. En este caso, los valores ajustados para las constantes han sido $A_{C V}=63, B_{C V}=60, C_{C V}=35 \mathrm{y}$ $D_{C V}=65$. Dicho ajuste se puede observar en la Figura 4.14. A pesar de que el ensayo Charpy no determina directamente la tenacidad a fractura del material, puede ser usado como una primera aproximación para estimarla. En la bibliografía están disponibles diversas correlaciones empíricas entre la tenacidad a fractura y los resultados del ensayo Charpy, recogidas la mayoría de ellas en el código ASME API 579. La utilización de unas o de otras depende principalmente del material utilizado y de la región de la curva que se desee correlacionar. En este caso se han usado las expresiones (4.11) y (4.12) facilitadas por el código ASME API 579. Los resultados obtenidos con ambas correlaciones se pueden ver en la Tabla 4.6, donde se ha recogido el valor medio para cada una de las temperaturas.

$$
\begin{aligned}
& \left(\frac{K_{I c}}{\sigma_{y s}}\right)^{2}=0.64 \cdot\left(\frac{C V N}{\sigma_{y s}}-0.01\right) \quad\left[M P a \cdot m^{1 / 2}, M P a, J\right] \\
& K_{I c}=14.6 \cdot(C V N)^{0.50} \quad\left[M P a \cdot m^{1 / 2}, M P a, J\right]
\end{aligned}
$$

Tabla 4.6. Estimación de $K_{I c}$, en función de la temperatura, según expresiones del código ASME API 579 [82].

\begin{tabular}{|c|c|c|c|c|c|c|c|c|c|}
\hline$K_{I c}$ & \multicolumn{10}{|c|}{ Temperatura $\left({ }^{\circ} \mathbf{C}\right)$} \\
\cline { 2 - 10 }$\left(\mathrm{MPa} \cdot \mathrm{m}^{1 / 2}\right)$ & $\mathbf{- 8 0}$ & $\mathbf{- 4 0}$ & $\mathbf{- 2 0}$ & $\mathbf{0}$ & $\mathbf{2 0}$ & $\mathbf{4 0}$ & $\mathbf{6 0}$ & $\mathbf{8 0}$ & $\mathbf{1 2 0}$ \\
\hline$(4.2)$ & - & 54.25 & 131.5 & 110.1 & 176.1 & 203.1 & 232.4 & 247.8 & 266.9 \\
\hline$(4.3)$ & 28.46 & 57.14 & 87.1 & 77.9 & 108.5 & 121.8 & 136.8 & 144.8 & 154.6 \\
\hline
\end{tabular}




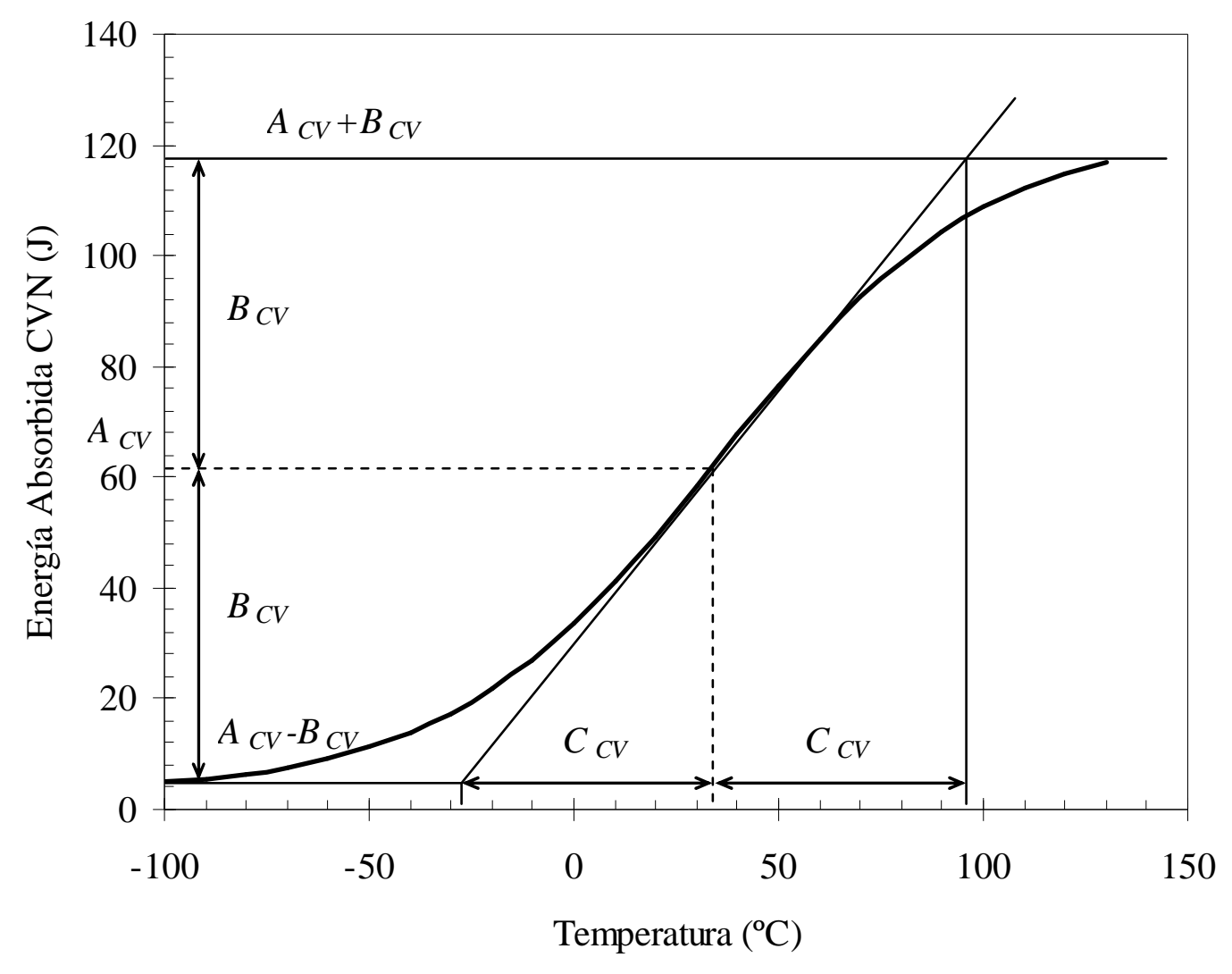

Figura 4.13. Esquema para determinar las constantes de la expresión (4.10) [82].

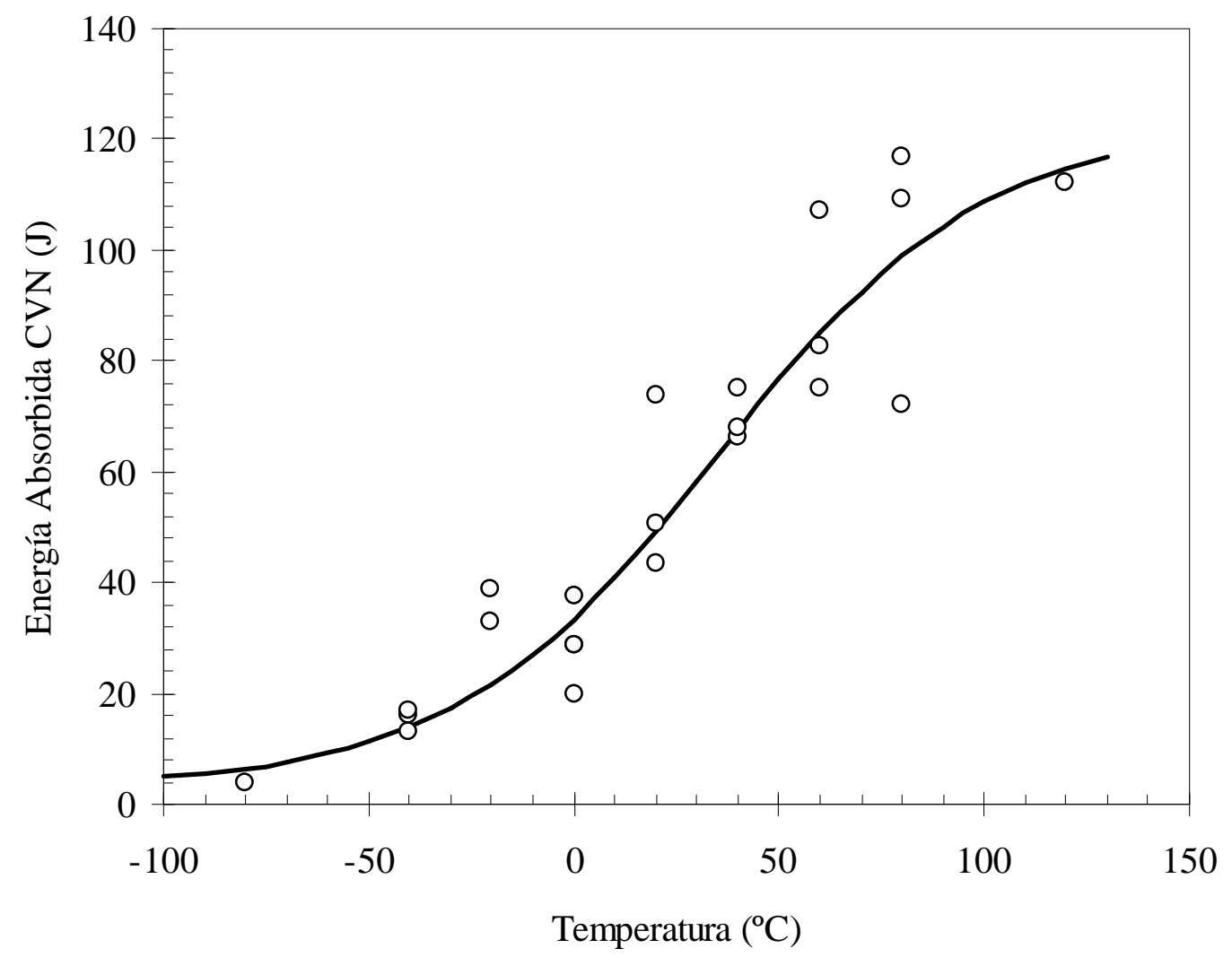

Figura 4.14. Curva transición dúctil-frágil del acero 15-5 PH. 


\subsection{TENACIDAD A FRACTURA}

Este tipo de ensayo permite determinar la tenacidad a fractura de materiales metálicos usando los parámetros $K, J$ y $C T O D$ en el Modo I de carga, utilizando probetas compactas (CT) prefisuradas por fatiga. La normas que rigen el desarrollo del ensayo y condiciones de la probeta son la ASTM E399 [112] y la ASTM E1820 [113].

Las probetas CT se han mecanizado según la dirección circunferencial, a partir de uno de los anillos de acero 15-5 $\mathrm{PH}$ que se posee. Al igual que en los apartados anteriores las dimensiones de este anillo son diámetro interior $275 \mathrm{~mm}$, diámetro exterior $385 \mathrm{~mm}$ y espesor $30 \mathrm{~mm}$. En la Figura 4.15 se recogen las principales dimensiones de las probetas CT siguiendo las recomendaciones de la norma ASTM E399.

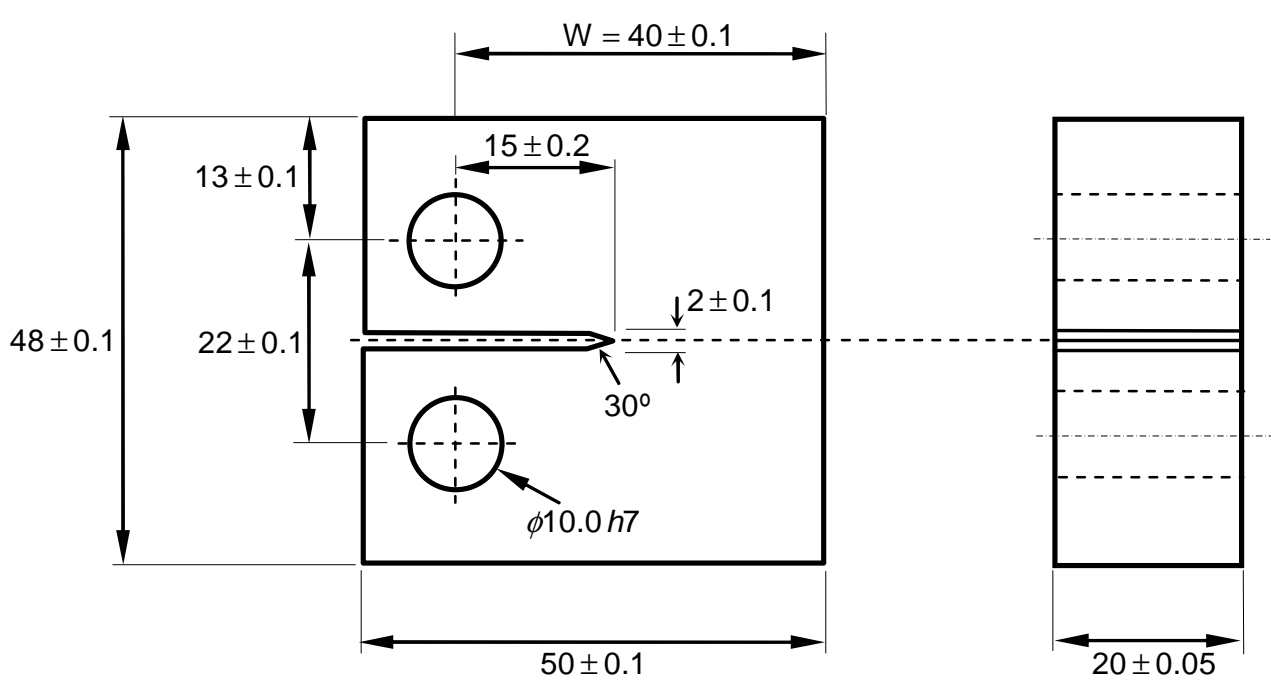

Figura 4.15. Dimensiones normalizadas de la probeta CT utilizada [112].

Los ensayos se han efectuado a temperatura ambiente, con una máquina de ensayo MTS 810 modelo 318.25, con un rango de fuerza hasta $250 \mathrm{kN}$. Se han realizado tres ensayos de tenacidad a fractura utilizando las probetas K3L2, K3L3 y K3L4.

Siguiendo el procedimiento de la norma, cada una de estas probetas se han prefisurado por fatiga, hasta obtener un valor inicial de fisura, cuyo valor debe estar comprendido entre $0.45 \cdot W \leq a_{o} \leq 0.70 \cdot W$ para la correcta evaluación de $J$ y del CTOD, y entre $0.45 \cdot W \leq a_{o} \leq 0.55 \cdot W$ para la determinación de $K_{I c}$ [113]. La Tabla 4.7 recoge los valores 
obtenidos de la fisura inicial para los tres ensayos realizados. Se puede observar como todos los valores de $a_{o}$ están dentro del rango válido para la evaluación de $J$ y del CTOD. También se puede comprobar como en las tres probetas el tamaño correspondiente a la fisura por fatiga cumple con los requisitos establecidos en la norma ASTM E1820.

Con el objetivo de reducir la sección resistente central de las probetas utilizadas se han mecanizado, posteriormente a la prefisuración por fatiga, dos entallas laterales en cada una de ellas, rebajando el espesor $B$ de la probeta en esta zona de $20 \mathrm{~mm}$ a $15.5 \mathrm{~mm}$. Este nuevo valor se denomina espesor neto $B_{N}$.

Tabla 4.7. Valores de fisura inicial en las probetas CT ensayadas.

\begin{tabular}{|c|c|c|}
\cline { 2 - 3 } \multicolumn{1}{c|}{} & \multicolumn{2}{c|}{ Fisura inicial } \\
\hline Probeta CT & $a_{o}(\mathrm{~mm})$ & $a_{o} / W$ \\
\hline K3L2 & 25.995 & 0.65 \\
\hline K3L3 & 23.975 & 0.60 \\
\hline K3L4 & 24.069 & 0.60 \\
\hline
\end{tabular}

Una vez preparadas las probetas se efectuaron los ensayos recogiendo los valores de la carga $(P$ ) y del desplazamiento en la línea de carga (COD). La longitud de fisura en cada instante ( $a$ ) se obtuvo mediante el método de las descargas elásticas.

Para cuantificar el valor de la longitud final de grieta en cada una de las probetas se ha llevado a cabo un análisis fractográfico de las superficies de fractura, los resultados obtenidos se muestran en la Tabla 4.8. Cada uno de los valores de la fisura final $a_{p}$ recogidos en la misma Tabla 4.8 se han obtenido a partir de la media aritmética de nueve medidas equidistantes realizadas sobre la superficie de fractura de cada probeta, cada una de estas medidas no difiere en más de un 5\% del valor de la media, tal y como prescribe la norma ASTM E1820 para que el ensayo sea válido, este hecho se puede apreciar también en las imágenes de la Tabla 4.8 en las que se ve cómo la forma de la fisura final es una elipse muy suavizada, pudiéndose asemejar prácticamente a una recta, lo cuál indica que la fisura ha propagado uniformemente por todo el frente de grieta. 
Posteriormente, se procede a analizar tanto las curvas P-COD como las curvas cargadesplazamiento de la línea de carga (LLD). Estas últimas se pueden observar en la Figura 4.16. En ambos casos se llega a la conclusión de que la fisura en las probetas K3L3 y K3L4 no se ha propagado lo suficiente como para considerar un crecimiento estable de fisura, produciéndose la rotura inestable de la probeta. Mientras que en la probeta K3L2 si que se puede hacer dicha consideración ya que la rotura de la probeta ha tenido lugar después del crecimiento dúctil y estable de grieta.

Tabla 4.8. Valores de fisura final en las probetas CT ensayadas.

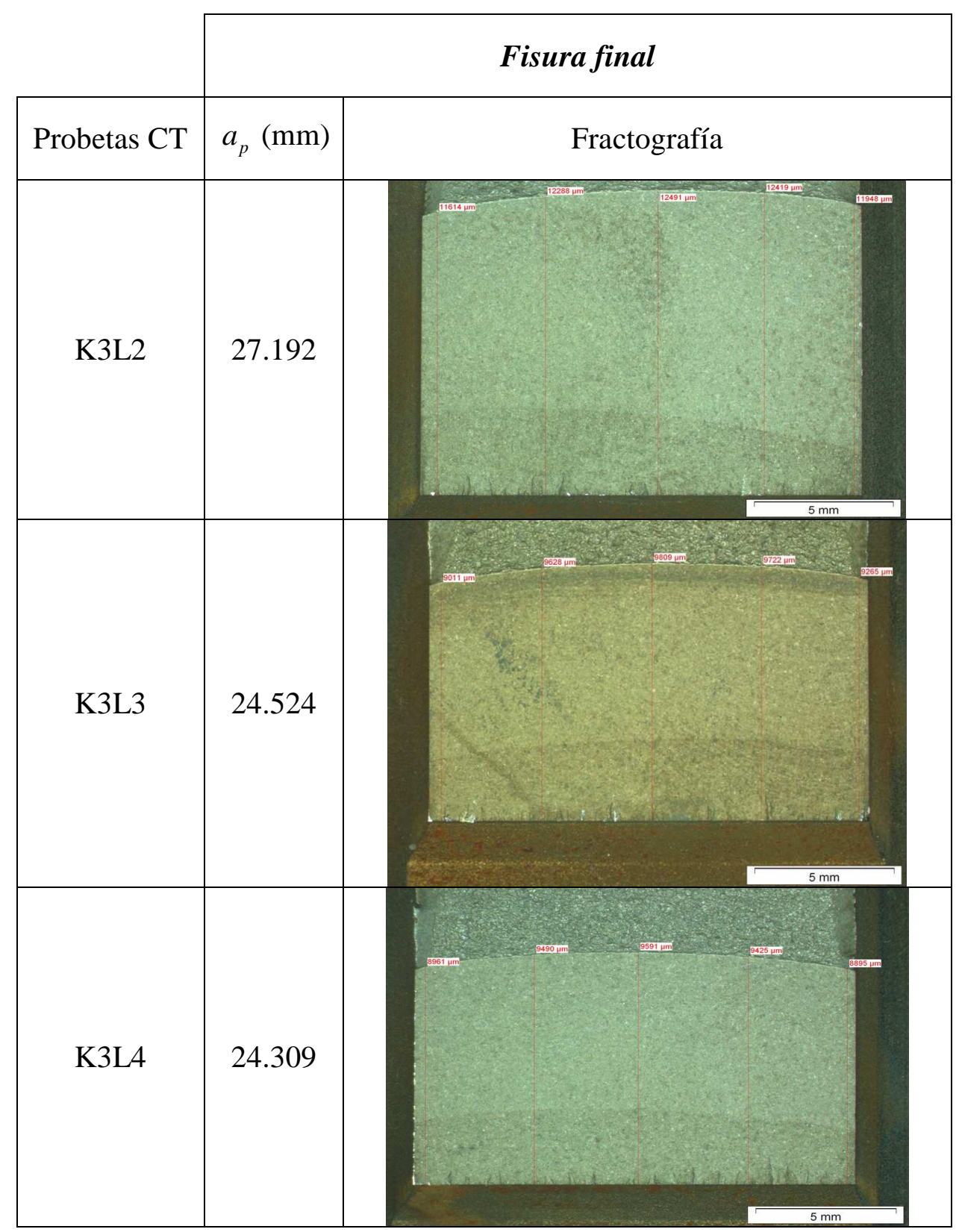




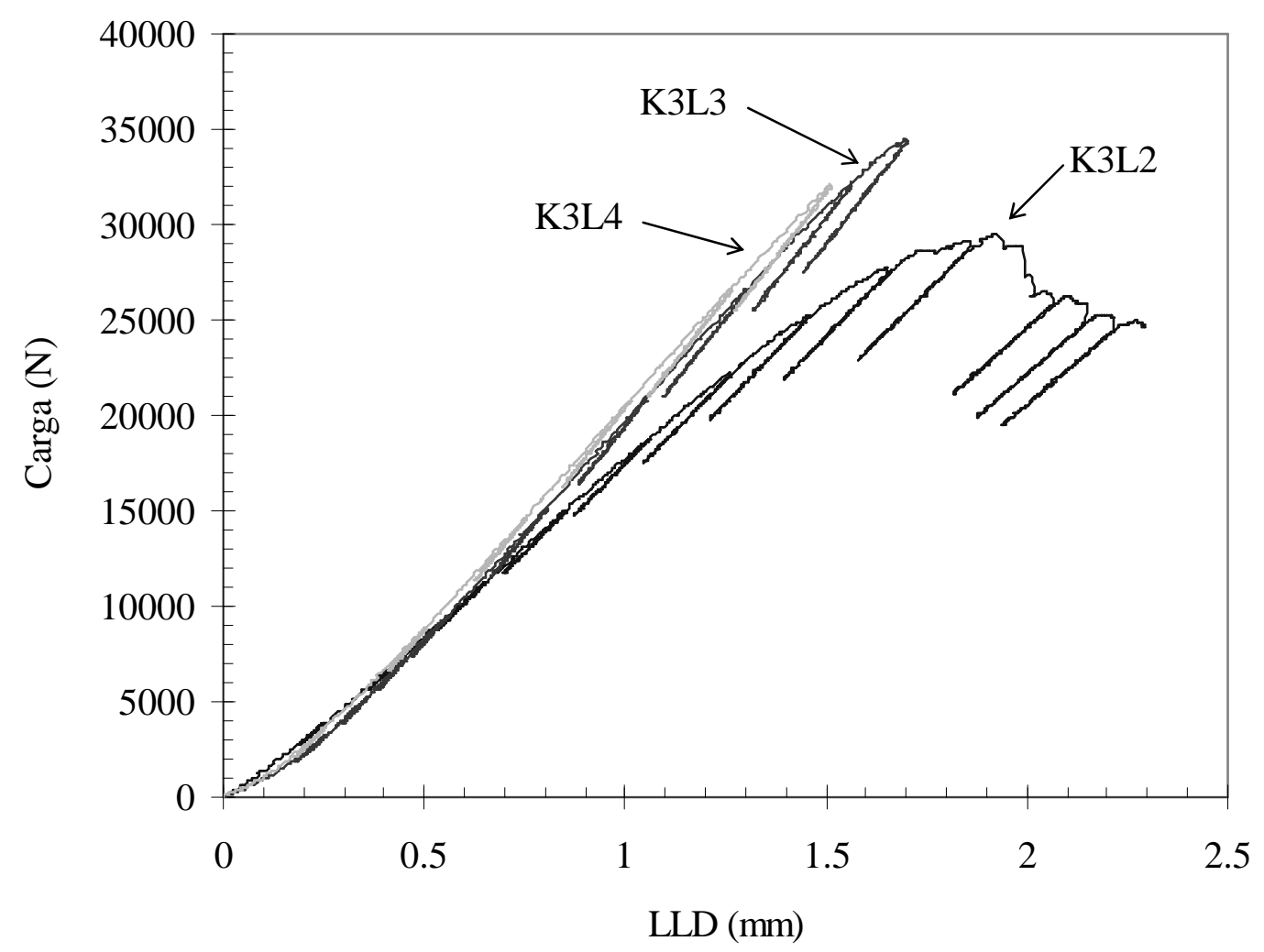

Figura 4.16. Curvas Carga-LLD de las probetas CT utilizadas.

Una vez realizada la fase experimental sobre cada probeta, se obtiene la integral $J$, para cada valor de $a$, mediante las recomendaciones de la norma ASTM E1820, a través de la expresión (4.13).

$J=\frac{\eta \cdot U}{B_{N} \cdot\left(W-a_{o}\right)} \cdot\left[1-\frac{(0.75 \cdot \eta) \cdot \Delta a}{W-a}\right]$

Donde $U$ representa la energía bajo la curva $P-C O D, B_{N}$ es el espesor neto de la probeta, y $\Delta a$ y $\eta$ vienen dados por las relaciones (4.14) y (4.15) respectivamente.

$\eta=2+0.522 \cdot\left(1-\frac{a_{o}}{W}\right)$

$\Delta a=a-a_{o}$

Finalmente, con los valores obtenidos, se representa la curva $J_{R}, \mathrm{o} J-\Delta a$. 
La Figura 4.17 muestra las curvas $J-\Delta a$ obtenidas en los ensayos, donde se puede observar también claramente como la fisura correspondiente a la probeta K3L2 es la única que ha crecido de forma estable. Para el caso de las probetas K3L3 y K3L4, donde el fallo se ha producido antes del crecimiento estable de la fisura, es posible obtener un valor de tenacidad denominado $J_{Q c}$, calculado en el punto final de la curva $J-\Delta a$ correspondiente de la Figura 4.17, además, en el caso de que se cumplan las condiciones necesarias definidas por las expresiones (4.16) y (4.17) [113], este valor de tenacidad se denomina $J_{c}$ y representa una medida de la tenacidad cuando se produce la inestabilidad sin apenas un crecimiento estable de la fisura, este parámetro es independiente de las dimensiones del plano de fisura, sin embargo puede haber cierta dependencia de esta tenacidad con el espesor, es decir, con la longitud del frente de grieta.

$$
\begin{aligned}
& B, b_{o} \geq 100 \cdot J_{Q} / \sigma_{y} \\
& \Delta a_{p}<0.2 m m+J_{Q} / M \cdot \sigma_{y}
\end{aligned}
$$

En las expresiones anteriores $B$ es el ancho o espesor de probeta, al tener las probetas entallas laterales se toma el valor del espesor efectivo $B_{e}$ definido por $B-\left(B-B_{N}\right)^{2} / B, b_{o}$ es el ligamento remanente inicial definido por $\mathrm{W}-a_{o}, \Delta a_{p}$ es el incremento de la fisura en el ensayo definido por $a_{p}-a_{o}, J_{Q}$ es el valor de $J$ en el punto de evaluación, en este caso $J_{Q c}$, $\sigma_{y}$ el valor del límite elástico $\sigma_{0.2}$ determinado en el ensayo de tracción y $M$ es una constante cuyo valor es 2 [113].

La Tabla 4.9 recoge los valores calculados para comprobar si los valores de $J_{Q c}$ de las probetas K3L3 y K3L4 pueden ser considerados como valores de tenacidad $J_{c}$. Es importante recordar que aunque se cumplan las condiciones necesarias definidas anteriormente, $J_{c}$ puede depender de la longitud del frente de grieta.

A partir de los resultados obtenidos en la Tabla 4.9 solamente el valor de $J_{Q c}$ de la probeta K3L4 se puede considerar como valor de tenacidad $J_{c}$, ya que cumple las tres condiciones necesarias recogidas en dicha tabla. 


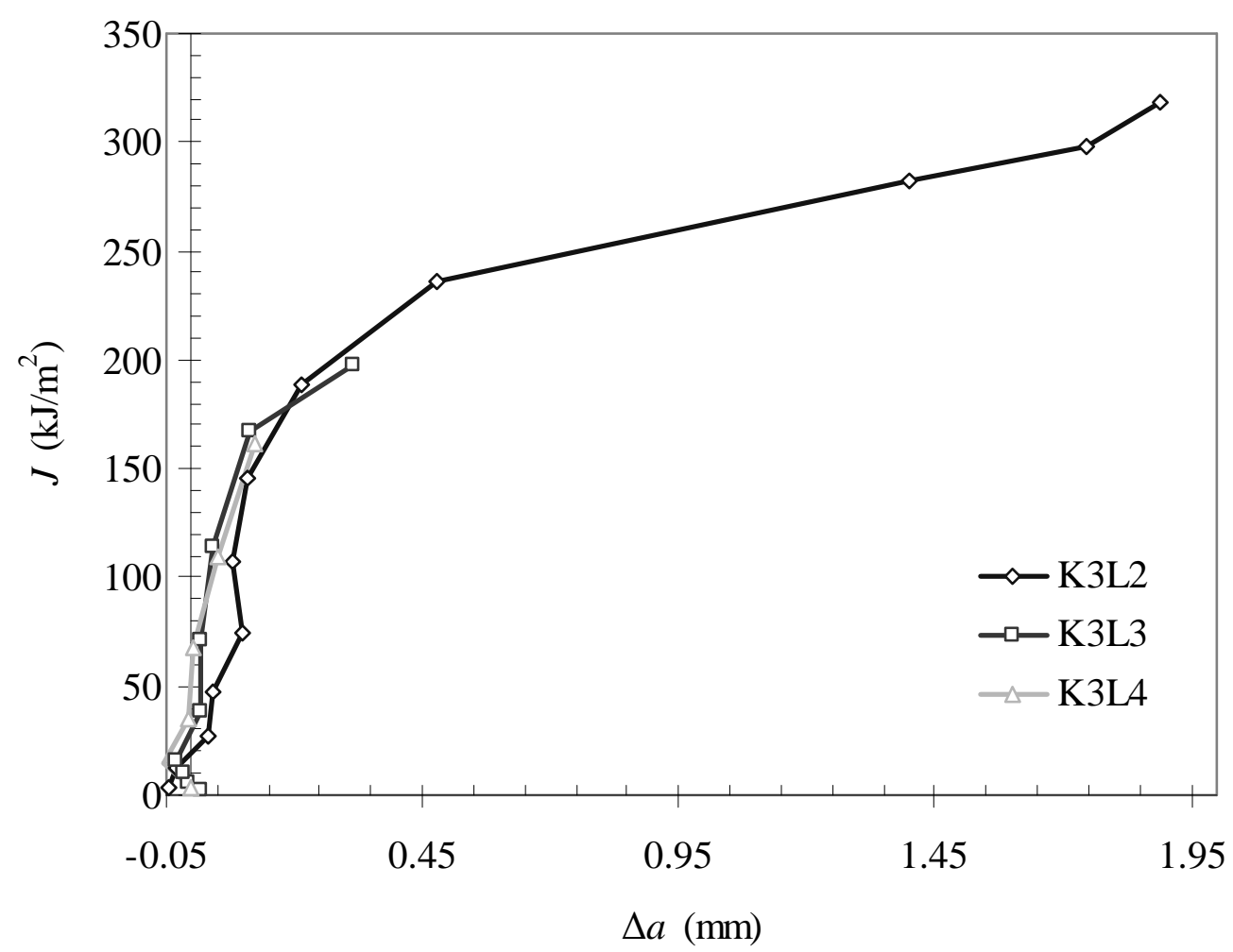

Figura 4.17. Curvas $J$ - $\Delta a$ de las probetas CT utilizadas.

Los resultados obtenidos en el ensayo de la probeta K3L2, donde se ha producido un crecimiento estable de la fisura, se van a analizar conforme marca la norma [113] para la obtención de $J_{I c}$. El primer paso es desechar los puntos de la curva $J-\Delta a$ que no se encuentran dentro del área definida por las rectas $\Delta a=0, J=0, \Delta a_{\max }=0.25 \cdot b_{o}$ y el menor valor de $J_{\max }=b \cdot \sigma_{y} / 20$ ó $J_{\max }=B \cdot \sigma_{y} / 20$.

Una vez identificados estos puntos, se procede a representar la "blunting line" acorde con la expresión (4.18), donde $M$ vuelve a ser una constante cuyo valor es 2 [113]. Seguidamente se trazan tres líneas de exclusión, dos de las cuáles son paralelas a la "blunting line” y cortan al eje de abscisas en los puntos 0.15 y $1.5 \mathrm{~mm}$, y la tercera es una línea horizontal que marca el valor de $J$ límite y está definida por la expresión (4.19).

$J=M \cdot \sigma_{y} \cdot \Delta a$

$J_{\text {limit }}=b_{o} \cdot \sigma_{y} / 15$ 
Tabla 4.9. Valores para la comprobación de $J_{c}$.

\begin{tabular}{|c|c|c|}
\hline \multirow[b]{2}{*}{ Parámetros } & \multicolumn{2}{|c|}{ Probetas $C T$} \\
\hline & K3L3 & K3L4 \\
\hline$B(m m)$ & 20 & 20 \\
\hline$B_{N}(m m)$ & 15.5 & 15.5 \\
\hline$B_{e}(m m)$ & 18.988 & 18.988 \\
\hline$W(\mathrm{~mm})$ & 40 & 40 \\
\hline$a_{o}(\mathrm{~mm})$ & 23.975 & 24.069 \\
\hline$a_{p}(m m)$ & 24.524 & 24.309 \\
\hline$\sigma_{y}(M P a)$ & 1100 & 1100 \\
\hline$J_{Q c}\left(k J / m^{2}\right)$ & 198.1 & 161.6 \\
\hline$M$ & 2 & 2 \\
\hline$b_{o}(m m)$ & 16.025 & 15.931 \\
\hline$\Delta a_{p}(m m)$ & 0.549 & 0.240 \\
\hline $100 \cdot J_{Q} / \sigma_{y}$ & 18.01 & 14.69 \\
\hline $0.2 m m+J_{Q} / M \cdot \sigma_{y}$ & 0.290 & 0.273 \\
\hline$B_{e} \geq 100 \cdot J_{Q} / \sigma_{y}$ & $\mathrm{Si}$ & $\mathrm{Si}$ \\
\hline$b_{o} \geq 100 \cdot J_{Q} / \sigma_{y}$ & No & $\mathrm{Si}$ \\
\hline$\Delta a_{p}<0.2 m m+J_{Q} / M \cdot \sigma_{y}$ & No & $\mathrm{Si}$ \\
\hline
\end{tabular}

Los puntos de la curva $J-\Delta a$ que están dentro del área encerrada por las líneas de exclusión anteriores y el eje de abscisas son los utilizados para ajustar una curva de regresión definida por la expresión (4.20) denominada curva $J-R$, donde $C_{1}$ y $C_{2}$ son constantes y $k_{2}=1$, al menos cinco puntos tienen que estar dentro de esta región para poder obtener un valor de $J_{I c}$ válido [113].

$J=C_{1} \cdot\left(\frac{\Delta a}{k_{2}}\right)^{C_{2}}$

La intersección entre esta curva de regresión y una recta paralela a la "blunting line" que corta al eje de abscisas en $0.2 \mathrm{~mm}$ definen un único punto de corte, de coordenadas $\left(\Delta a_{Q}, J_{Q}\right)$. El 
valor de $J_{Q}$ se puede considerar como $J_{I c}$ si se cumplen una serie de condiciones necesarias [113]. En la Figura 4.18 se pueden ver los puntos de la curva $J-\Delta a$ de la probeta K3L2 que se encuentran dentro de la región válida de puntos definida anteriormente (puntos negros en la figura).

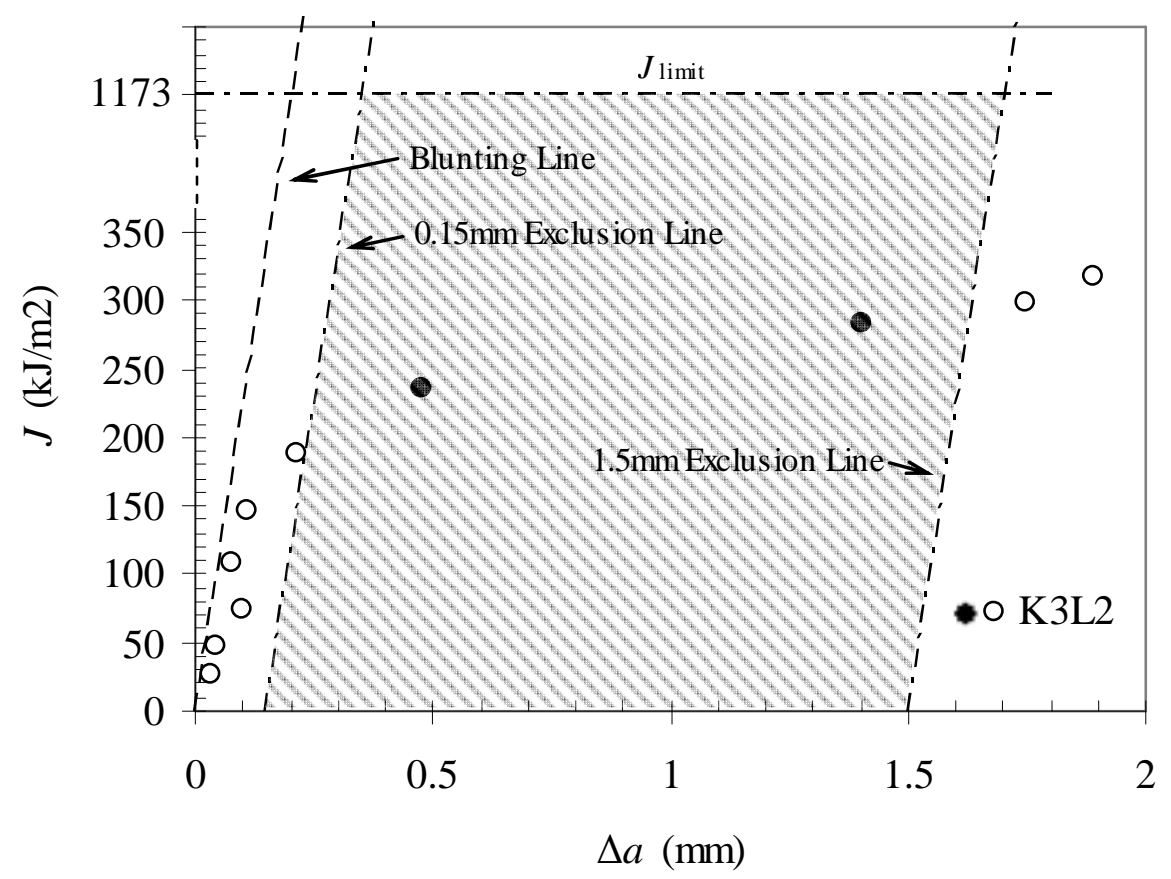

Figura 4.18. Región válida para la obtención de $J_{I c}$.

A pesar de que según la norma ASTM E1820 debemos tener al menos cinco puntos calculados en la zona de validez para tener un ajuste que permita calcular $J_{I c}$, la tendencia de los puntos anteriores y posteriores hace suponer que el ajuste realizado en este caso es bueno. De este modo, se obtiene finalmente una curva de regresión de ecuación $J=267.45 \cdot \Delta a^{0.249}$ (Figura 4.19), la cuál define un único punto de corte, de coordenadas $(0.29,196.5)$, con la recta paralela a la "blunting line" que corta al eje de abscisas en $0.2 \mathrm{~mm}$, por lo cuál, se obtiene para la probeta K3L2 el valor de $J_{I c} \approx J_{Q}=196.5 \mathrm{~kJ} / \mathrm{m}^{2}$.

A partir de los diferentes valores de $J$ determinados para cada una de las probetas ensayadas, es posible obtener un valor relacionado de $K$ a través de la expresión (4.21).

$$
K=\sqrt{\left(E^{\prime} \cdot J\right)}
$$


Donde $E^{\prime}=E /\left(1-v^{2}\right)$, sustituyendo los valores correspondientes al acero 15-5 PH en la expresión anterior se obtienen los valores de $K$ recogidos en la Tabla 4.10 para cada una de las probetas ensayadas.

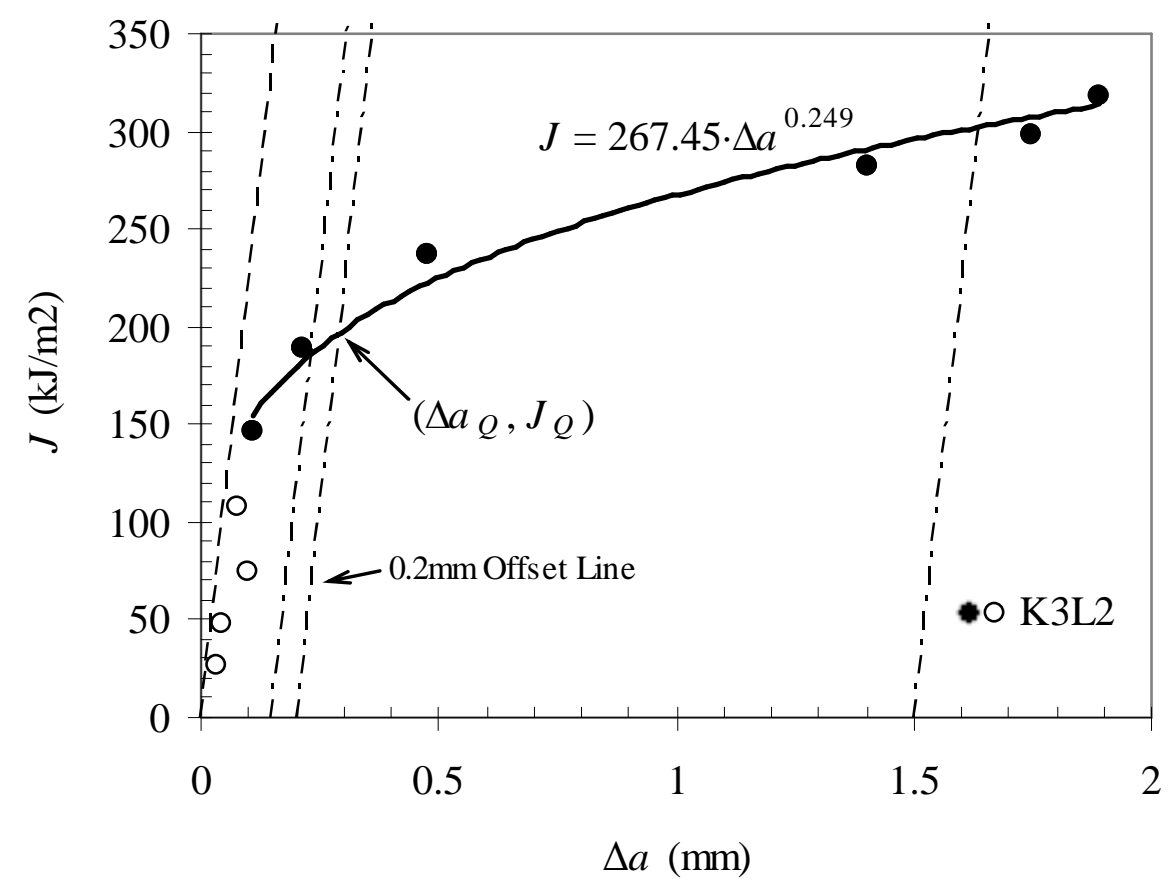

Figura 4.19. Curva $J-R$ de la probeta $K 3 L 2$.

Tabla 4.10. Valores de $J$ y $K$ para las probetas CT utilizadas.

\begin{tabular}{|c|c|c|c|}
\cline { 2 - 4 } \multicolumn{1}{c|}{} & \multicolumn{3}{c|}{ Probetas CT } \\
\hline Parámetros & $\mathrm{K} 3 \mathrm{~L} 2$ & $\mathrm{~K} 3 \mathrm{~L} 3$ & $\mathrm{~K} 3 \mathrm{~L} 4$ \\
\hline$J\left(\mathrm{~kJ} / \mathrm{m}^{2}\right)$ & $J_{Q}=196.5$ & $J_{Q c}=198.1$ & $J_{c}=161.6$ \\
\hline$K\left(\mathrm{MPa} \cdot \mathrm{m}^{1 / 2}\right)$ & 203.3 & 204.1 & 184.4 \\
\hline
\end{tabular}

Con los valores recogidos en la Tabla 4.10 y en la Tabla 4.6, se puede establecer un rango de variación de la tenacidad del material $K$ suficientemente aproximado para el objetivo final de la presente Tesis. Dicho rango de valores servirá para su posterior comparación con los resultados de $K_{\text {mat }}$ estimados mediante el procedimiento de ensayo de probetas SPT prefisuradas, desarrollado en la presente Tesis. 


\subsection{CONSIDERACIONES FINALES}

El material utilizado como base para el estudio y validación de la metodología desarrollada en la presente Tesis ha sido el acero 15-5 PH, de elevado límite elástico, endurecido por precipitación. El material, a priori, presenta una buena isotropía, ya que tanto los valores de dureza y los de tensión última de tracción en las direcciones transversal y longitudinal son muy similares. Por otro lado, el elevado valor del parámetro $n$ indica que el acero 15-5 PH puede ser considerado como un acero con un comportamiento casi perfectamente plástico. La técnica de superficie de respuesta se puede aplicar a este acero, ya que la ley de comportamiento del material se ajusta perfectamente a una ecuación tipo Ramberg Osgood. Como se explica al final del apartado 4.3, los datos correspondientes a la probeta A2B1 son los que se han utilizado en la presente Tesis cuando ha sido necesario, debido a que todas las probetas SPT utilizadas se han obtenido en la dirección circunferencial, este hecho, en este caso no es del todo crucial ya que como se ha visto el acero 15-5 PH presenta una buena isotropía. Para solventar el problema, de la posible escasez de material para realizar ensayos de tracción, se ha presentado un procedimiento que permite determinar estas propiedades a partir de la curva del SPT. Dicho método, está basado en el estudio que realizaron Mao y Takahashi y en la simulación numérica. Dicho procedimiento se ha aplicado al acero 15-5 PH, estimando los parámetros elastoplásticos con un buen grado de aproximación.

Los resultados obtenidos en el ensayo Charpy pueden ser utilizados para realizar una primera aproximación del valor de tenacidad a fractura del acero 15-5 PH, para ello se han utilizado dos correlaciones empíricas disponibles en el código ASME API 579 [82]. Teniendo en cuenta conjuntamente los resultados de las dos correlaciones, para la temperatura ambiente el valor de $K_{I c}$ varía entre 108.5 y $176.1 \mathrm{MPa} \cdot \mathrm{m}^{1 / 2}$. A simple vista, se puede decir que este rango de variación es muy amplio, debido principalmente al uso de resultados provenientes de un ensayo dinámico, como es el ensayo Charpy, para estimar un parámetro estático como es la tenacidad a fractura del material. Este ensayo sirve como primera aproximación. Con el ensayo de tenacidad a fractura el rango de variación de la tenacidad para el acero 15-5 PH, oscila entre 184.4 y $204.1 \mathrm{MPa} \cdot \mathrm{m}^{1 / 2}$. Este rango de valores es lo suficientemente reducido como para considerarlo válido y tomarlo como referencia a la hora de efectuar las correspondientes comparaciones con los resultados que se obtengan de la aplicación de la metodología desarrollada. 


\section{CAPÍTULO 5}

\section{APLICACIÓN DE LA METODOLOGÍA Y ANÁLISIS DE RESULTADOS}

\subsection{INTRODUCCIÓN}

Una vez realizada la caracterización del material que se empleará en la presente Tesis, se procede a aplicar la metodología anteriormente desarrollada y a determinar el grado de exactitud que con ella puede llegar a obtenerse para predecir la tenacidad del material. Se recogen los resultados intermedios obtenidos, explicando cada uno de los pasos de dicha metodología, y estimando como paso final el valor de la tenacidad a fractura del material.

En el presente capítulo se ahonda en las particularidades que se presentan en la aplicación de la metodología propuesta. En particular, se analizarán las diferentes formas de determinar la iniciación de propagación de fisura en las probetas SPT prefisuradas. Los tres métodos presentados en el capítulo anterior son ampliamente analizados. También se analizará en profundidad cuál es el tamaño de fisura óptimo a mecanizar en las probetas SPT y cuál es su efecto en la provisión de la tenacidad del material. Finalmente, se aportan una serie de recomendaciones para obtener un valor de tenacidad que sea representativo del material analizado.

Las diversas alternativas propuestas para la aplicación de la metodología implican un elevado número de resultados en cada uno de los pasos intermedios. Para mejorar su seguimiento y comprensión se ha elaborado el esquema representado en la Figura 5.1. 


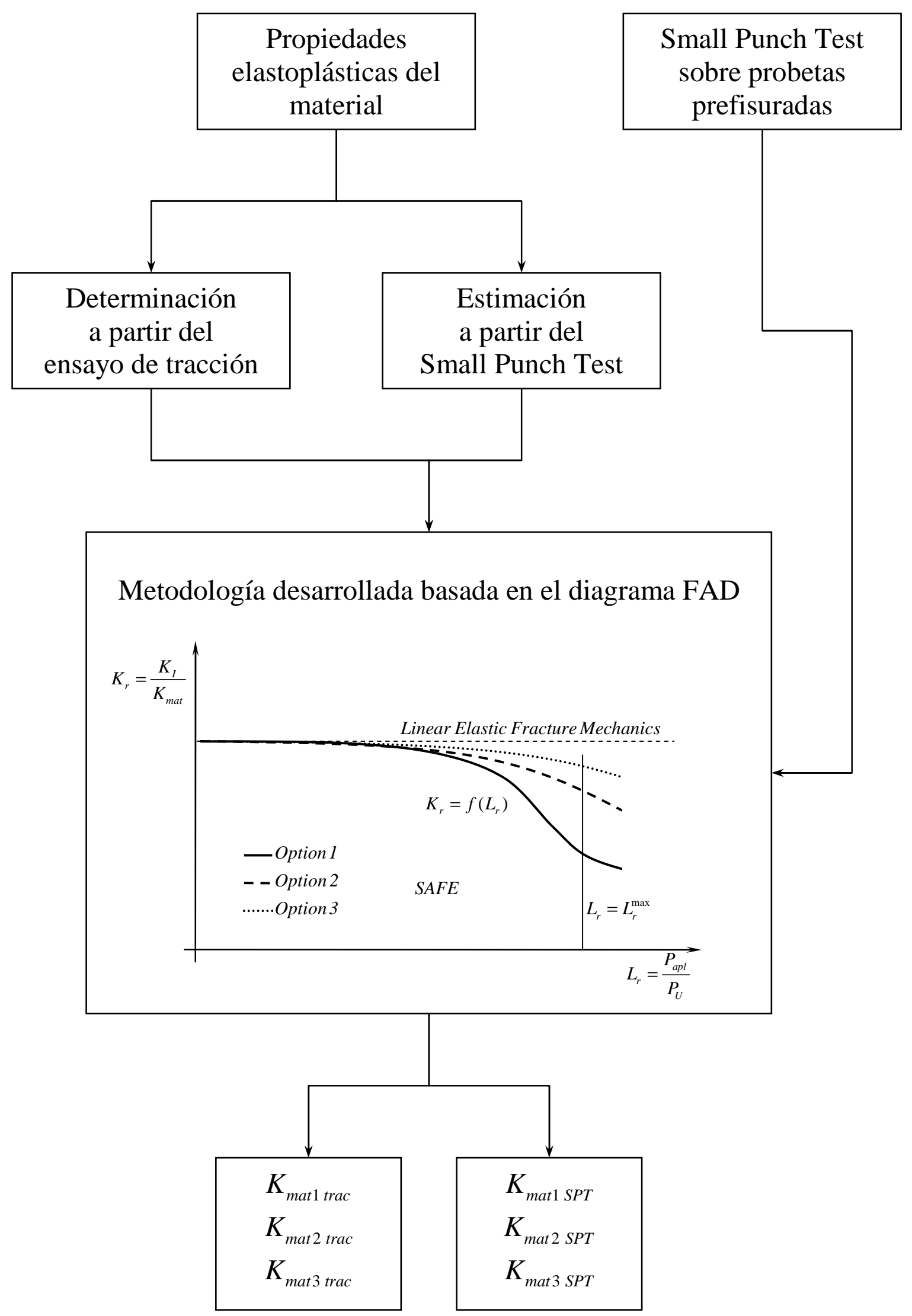

Figura 5.1. Esquema de aplicación de la metodología para cada probeta SPT prefisurada. 


\subsection{OBTENCIÓN DE LA CARGA DE INICIO DE PROPAGACIÓN, $P_{a p l}$}

Para la determinación de la carga aplicada sobre la probeta en el instante de inicio de propagación de fisura se han presentado, en el Capítulo 3, tres posibles métodos (ensayos interrumpidos, evaluación de la pendiente de la curva y método de la flexibilidad). Todos ellos se han empleado en la presente Tesis para determinar el método más adecuado, utilizando los datos generados tras el ensayo de las probetas SPT prefisuradas del acero 15-5 PH. En estas probetas la relación $a / t$ teórica ha sido de $0.2,0.3,0.4$ y 0.5.

Dicha relación se ha comprobado en todas ellas posteriormente al ensayo dado que estos valores teóricos pueden diferir de los reales, debido a la técnica de preparación de la fisura inicial (microfisuración por láser). Siempre que se emplee esta técnica de preparación de probetas SPT prefisuradas se recomienda realizar una comprobación de la profundidad real de fisura.

De los tres métodos comentados, el primero en emplearse ha sido el de los ensayos interrumpidos sobre probetas SPT prefisuradas con una relación $a / t$ teórica de 0.5 y 0.4 . Posteriormente se ha utilizado la técnica de evaluación de la pendiente de la curva para las probetas con relación $a / t$ teórica de $0.4,0.3$ y 0.2. Mientras que el método de la flexibilidad sólo se ha usado para las probetas con relación $a / t$ teórica de 0.4. Al final del apartado se recogen todos los valores de $P_{a p l}$ estimados con las técnicas comentadas.

\subsubsection{Ensayos interrumpidos sobre probetas SPT prefisuradas con relación a/t de 0.54}

Se ha realizado una primera batería de cinco ensayos interrumpidos para la determinación de la carga aplicada en el instante de inicio de la propagación de fisura, sobre las probetas SPT prefisuradas con relación $a / t$ real de 0.54. Las probetas empleadas en los ensayos interrumpidos del 1 al 5 se han denominado SPT0.5-01, SPT0.5-02, SPT0.5-03, SPT0.5-04 y SPT0.5-05 respectivamente. La Figura 5.2 muestra las curvas carga-desplazamiento del punzón obtenidas en cada uno de los ensayos, donde se puede observar como las curvas son prácticamente coincidentes. La forma de las curvas corresponde con la curva típica para una relación $a / t$ teórica mayor de 0.5 (Figura 3.4). 
La Figura 5.3 muestra la deformación alcanzada de la probeta en cada uno de los ensayos interrumpidos. En los ensayos 04 y 05 se observa claramente que la fisura ya se ha iniciado. Parece intuirse a simple vista la iniciación en el ensayo 03 y se desconoce lo ocurrido en 01 y 02. Para determinar con exactitud si se ha producido o no el inicio en los ensayos 01 a 03 se ha procedido a la fragilización con nitrógeno líquido de las probetas y a su posterior división en dos mitades.

Posteriormente se ha realizado un análisis fractográfico mediante microscopía electrónica de barrido (SEM) de las superficies de fractura para determinar con exactitud el punto de inicio de propagación de fisura, el cuál se encontrará entorno al ensayo interrumpido 03. Dicho análisis también será muy útil para determinar los mecanismos de fractura y la profundidad y forma exacta de la fisura en cada uno de los ensayos. Los resultados obtenidos con este análisis, para esta primera batería de ensayos SPT interrumpidos sobre probetas prefisuradas, se muestran a continuación.

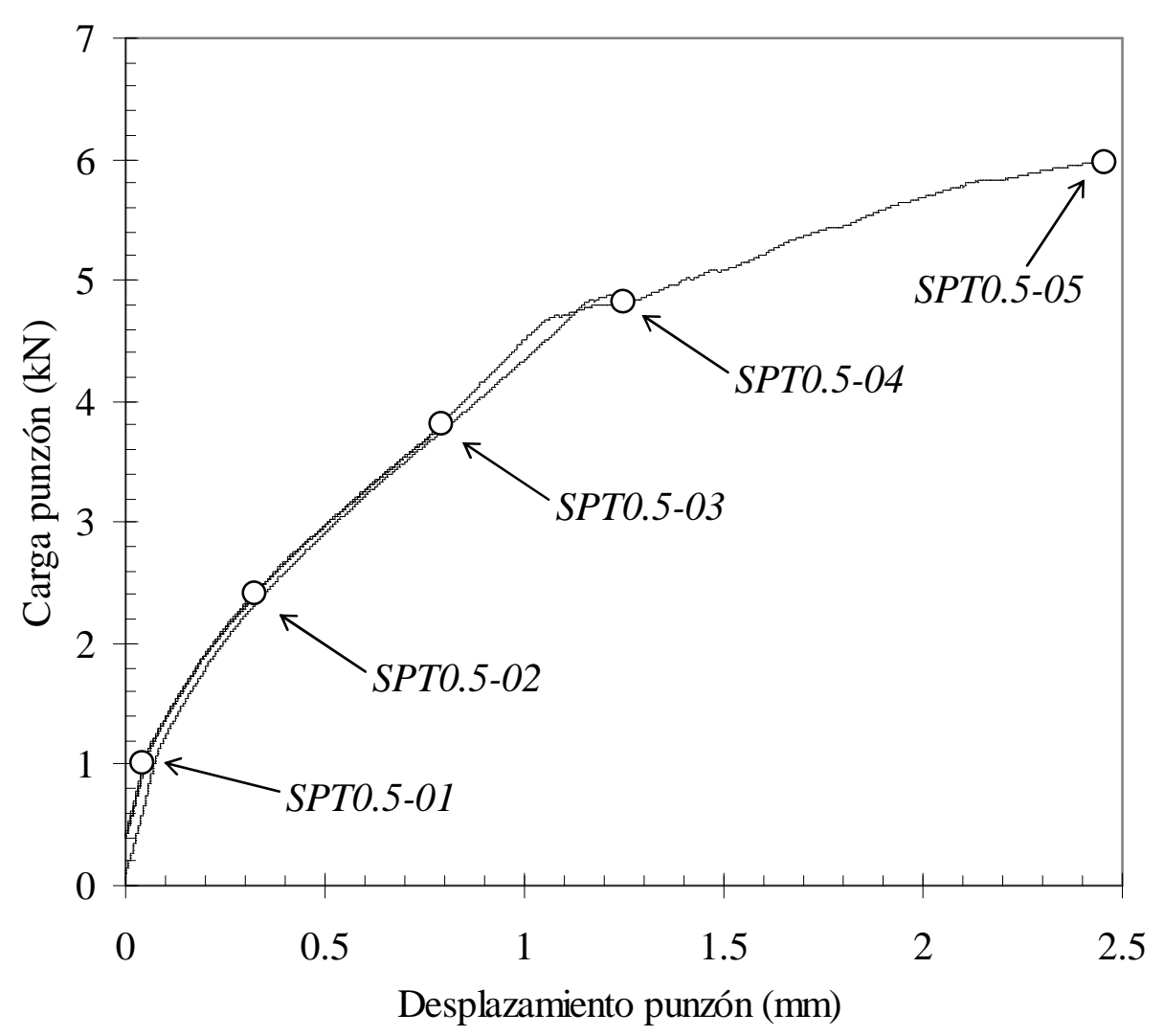

Figura 5.2. Curvas carga-desplazamiento del punzón obtenidas en los ensayos interrumpidos SPT sobre probetas con relación al t teórica de 0.5. 

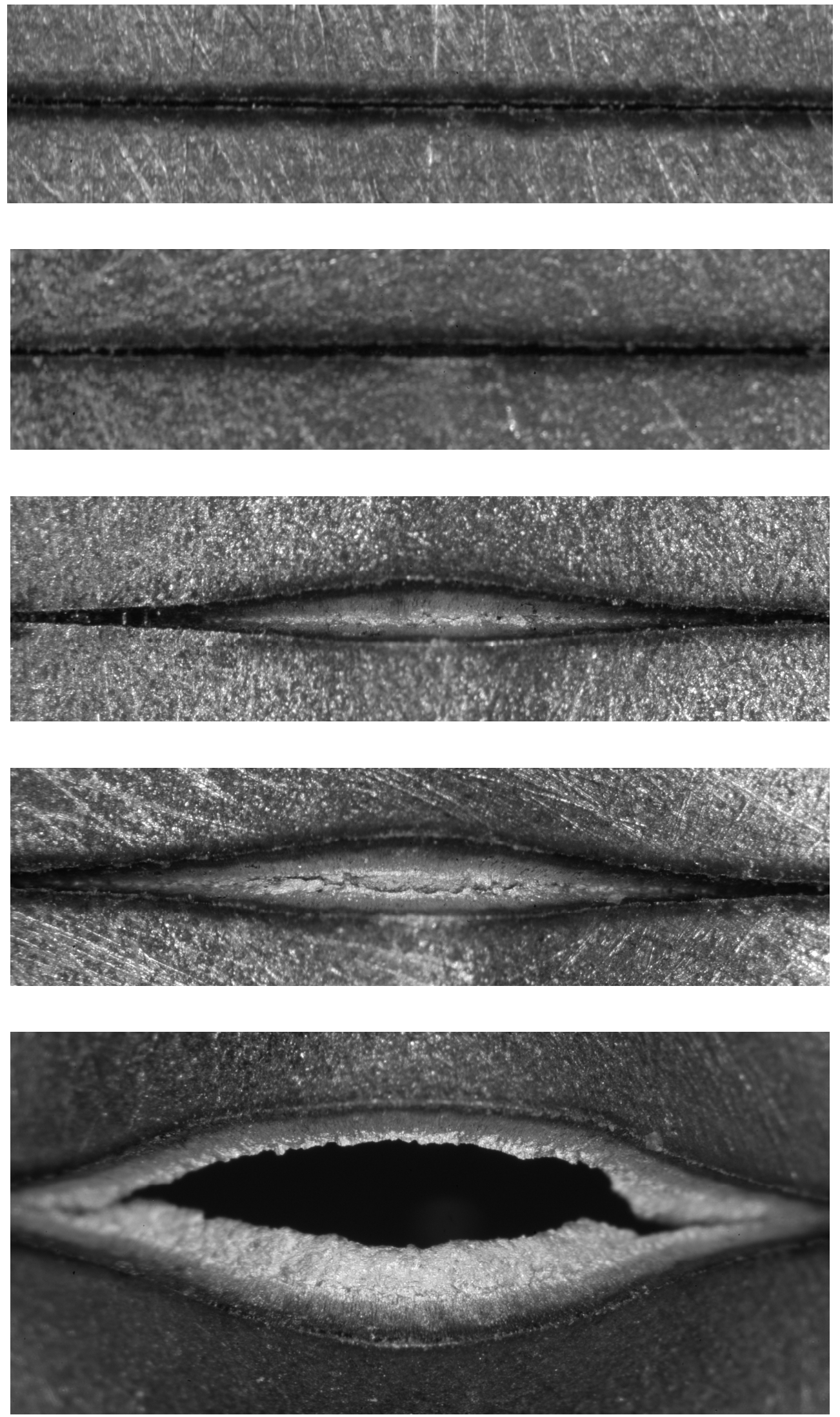

Figura 5.3. Evolución de la propagación de la fisura desde el ensayo interrumpido 01 al 05. 
En este primer análisis SEM, también se va a realizar la comprobación de la calidad de la microfisuración por láser. La Figura 5.4 muestra una metalografía de la fisura inicial realizada. En ella se puede observar claramente que la forma que tiene es de V, donde la punta de fisura es afilada, asemejándose a una fisura perfecta sin redondeo en la punta. En el detalle ampliado de la figura se puede identificar como quedan restos de material fundido y del propio material en la punta de fisura, esto es debido al propio láser, ya que se aplica a través de un haz de pulsos intermitentes, dejando aleatoriamente en la punta de fisura una serie de pequeños ligamentos, como se puede observar en las imágenes siguientes.

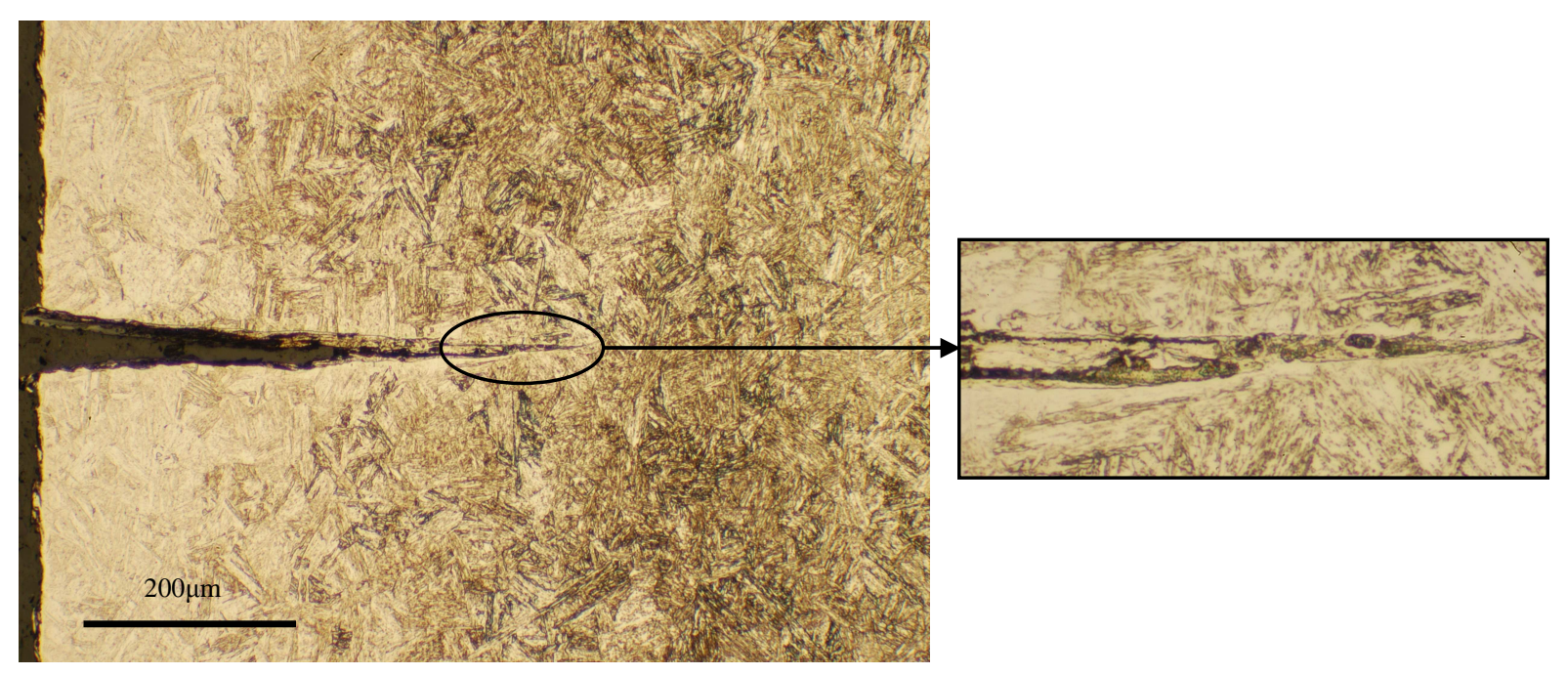

Figura 5.4. Metalografía de la fisura inicial.

En la Figura 5.5 y en la Figura 5.6 correspondientes al ensayo interrumpido 02 se puede observar la profundidad inicial de la fisura, ligeramente variable en longitud, debido a que el láser va dejando picos y valles a lo largo de la punta de fisura conforme va avanzando. Se puede distinguir claramente en las dos imágenes que la parte superior de la sección tiene el aspecto característico de una rotura frágil, debida al nitrógeno líquido utilizado para partir la probeta, y que en la parte inferior correspondiente a la fisura inicial aparecen una serie de marcas verticales debidas al haz de pulsos del láser. La diferencia entre las dos imágenes se encuentra en la zona central, ya que en la Figura 5.6 las dos partes anteriores están separadas por una pequeña franja de color más oscuro y aspecto liso, mientras que en la zona del empotramiento no se produce este fenómeno, tal y como se puede ver en el detalle de la Figura 5.7. 


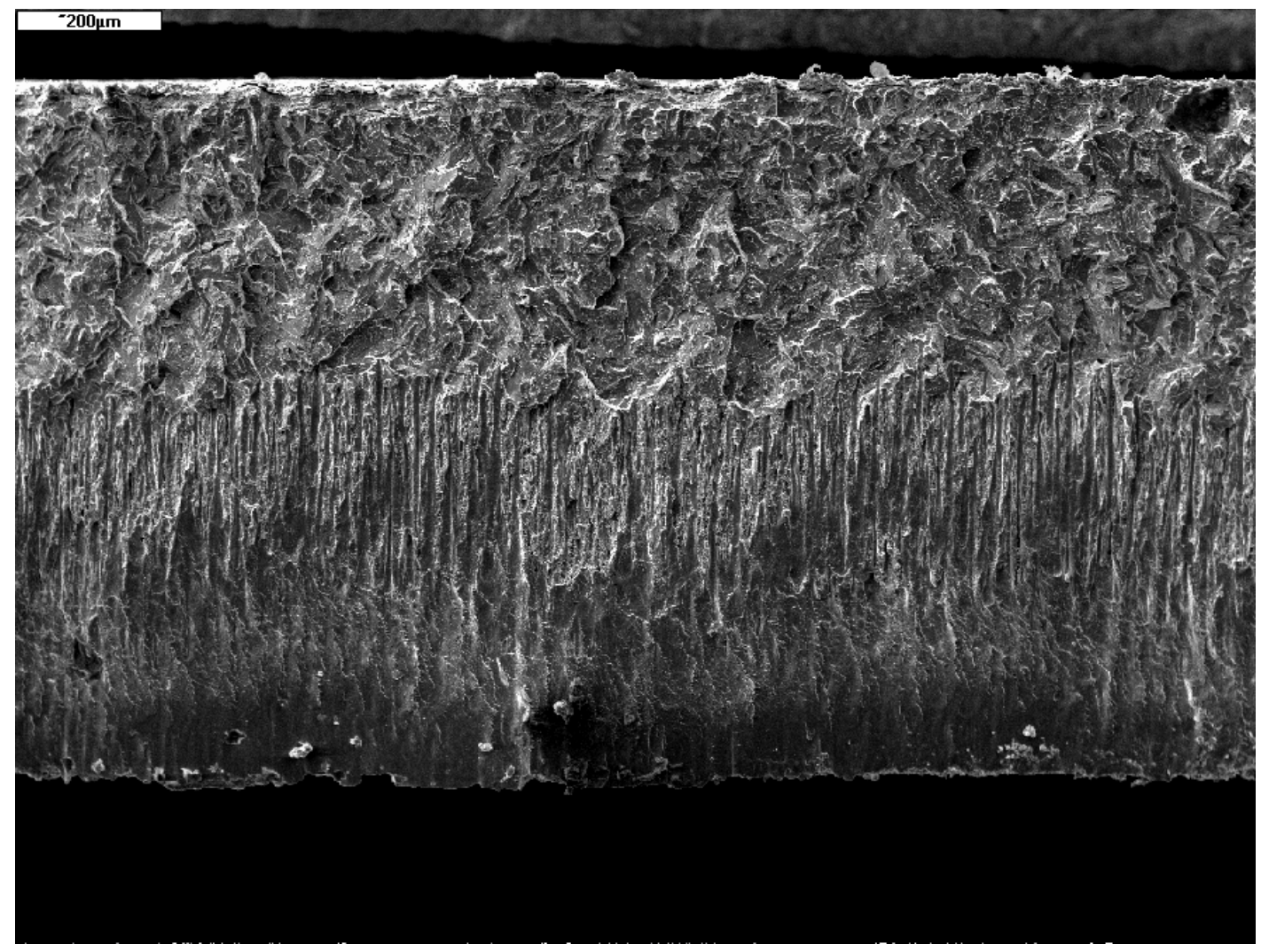

Figura 5.5. Zona empotrada de la fisura en la probeta SPT0.5-02 (75X).

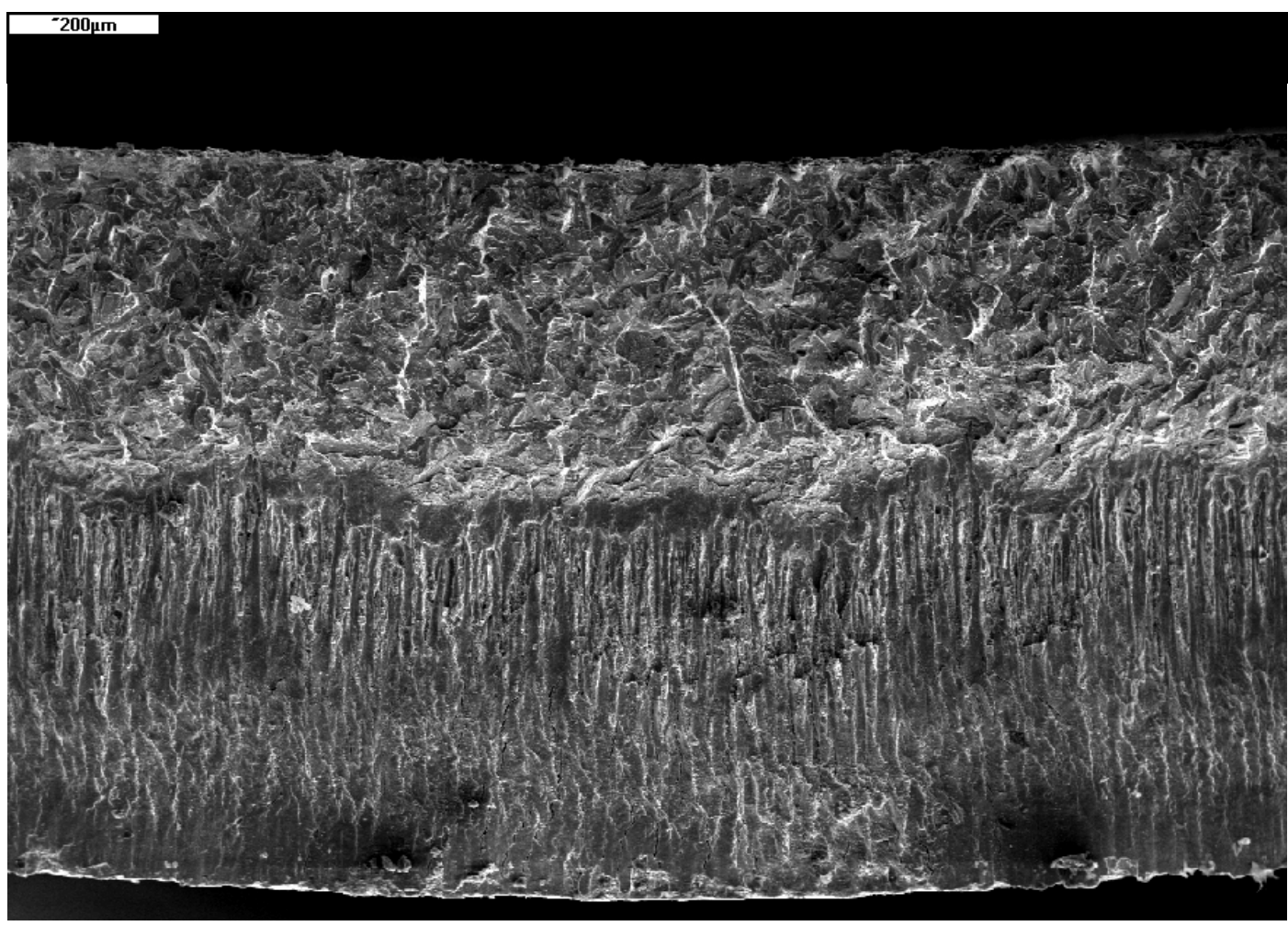

Figura 5.6. Zona central de la fisura en la probeta SPT0.5-02 (75X). 


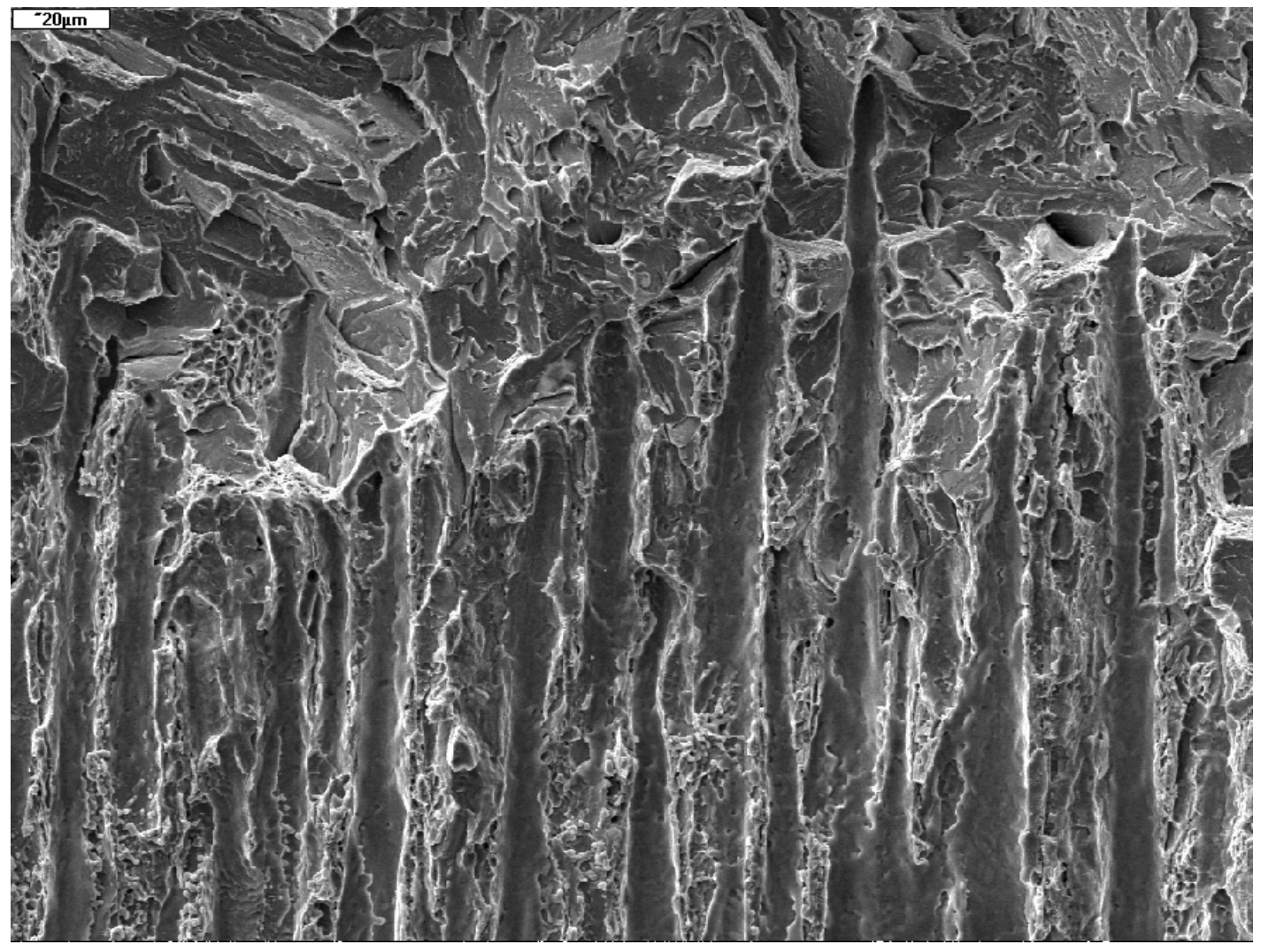

Figura 5.7. Detalle de la punta de fisura de la Figura 5.5 (500X).

Esta franja corresponde a los pequeños ligamentos de la punta de fisura, que se han roto durante el proceso de carga y aplastado probablemente durante el proceso de descarga, dando lugar a esa apariencia, como se puede ver en el detalle de la Figura 5.8. Una vez ocurrido el desgarro de estos ligamentos se obtiene una punta de fisura continua, a pesar de esto, todavía no se observan indicios de que haya iniciado la fisura en este punto, por lo que es necesario estudiar la sección del siguiente ensayo interrumpido, es decir, la correspondiente al ensayo interrumpido 03. En la Figura 5.9 perteneciente a dicha sección se puede apreciar, además de las partes correspondientes a la rotura frágil y a la fisura inicial, una parte central de color claro correspondiente al inicio de la propagación de la fisura a través de los mecanismos de fractura dúctil.

Estos mecanismos se pueden ver claramente en la Figura 5.10, en la que se observa como se ha producido la nucleación, crecimiento y coalescencia de microhuecos en la zona de inicio de propagación de la fisura. En la Figura 5.11 se puede apreciar la zona de transición de la fractura dúctil a la de fractura frágil producida al partir la probeta por fragilización con nitrógeno líquido. 


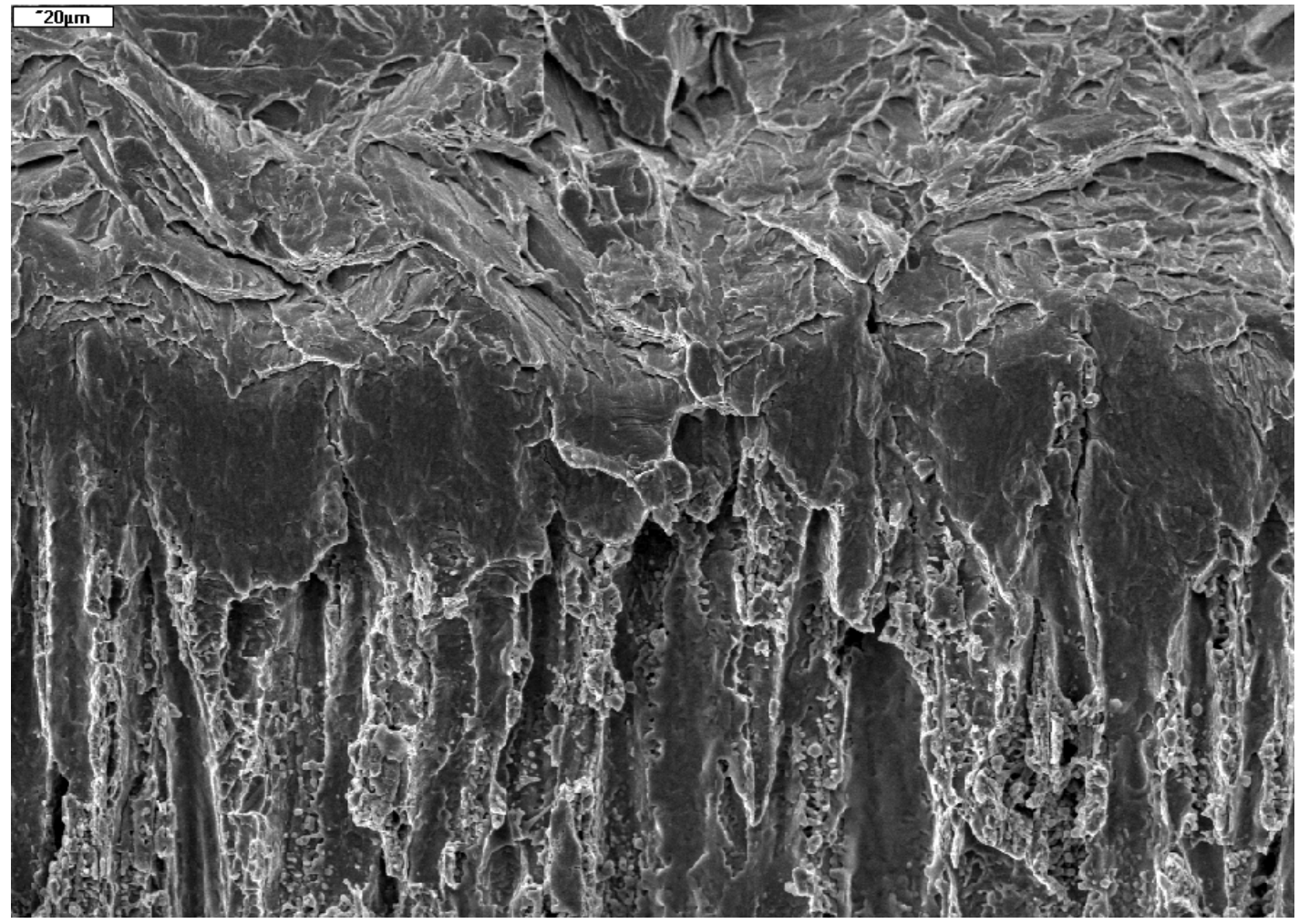

Figura 5.8. Detalle de la punta de fisura de la Figura 5.6 (500X).

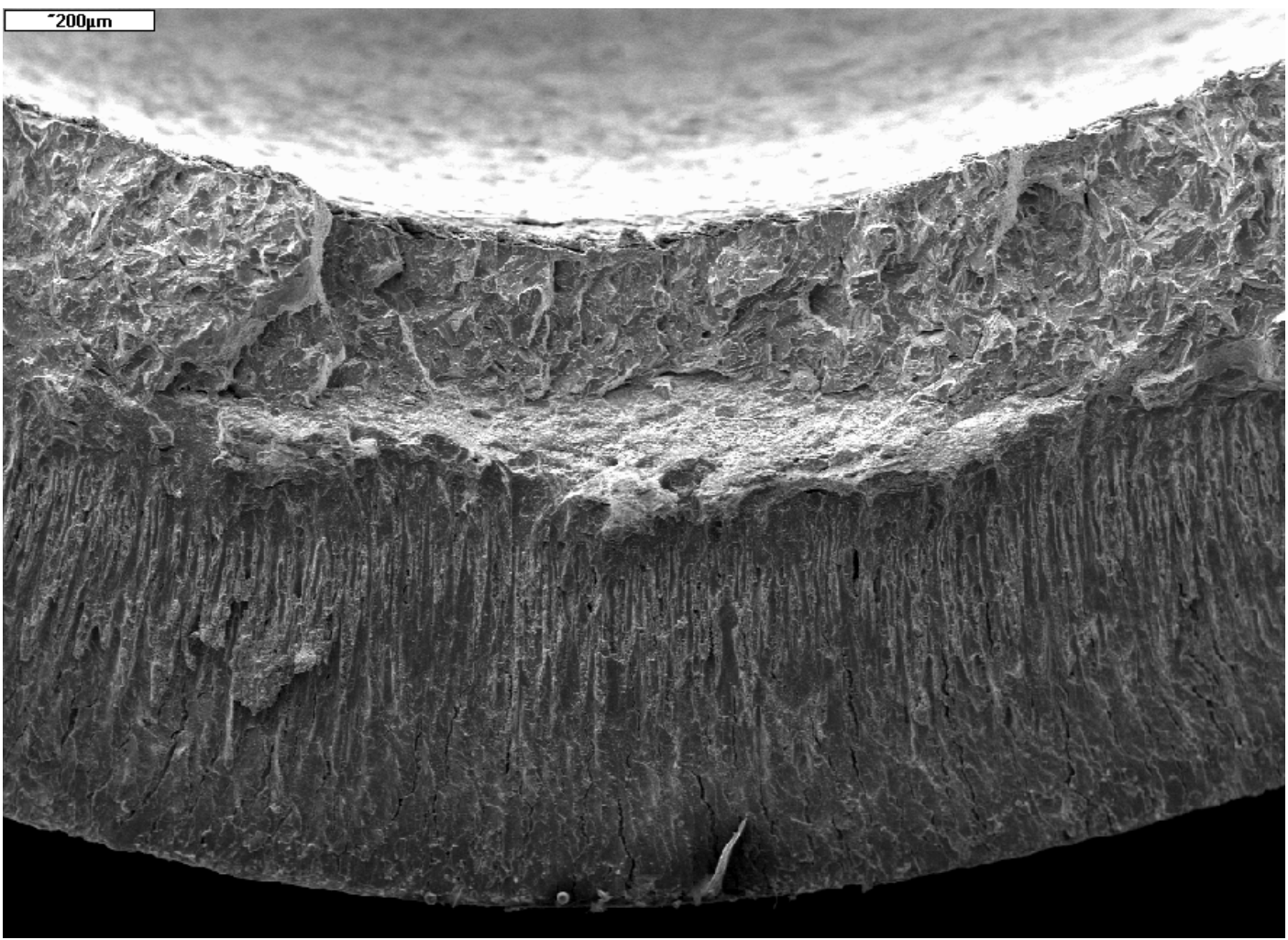

Figura 5.9. Zona central de la fisura en la probeta SPT0.5-03 (75X). 


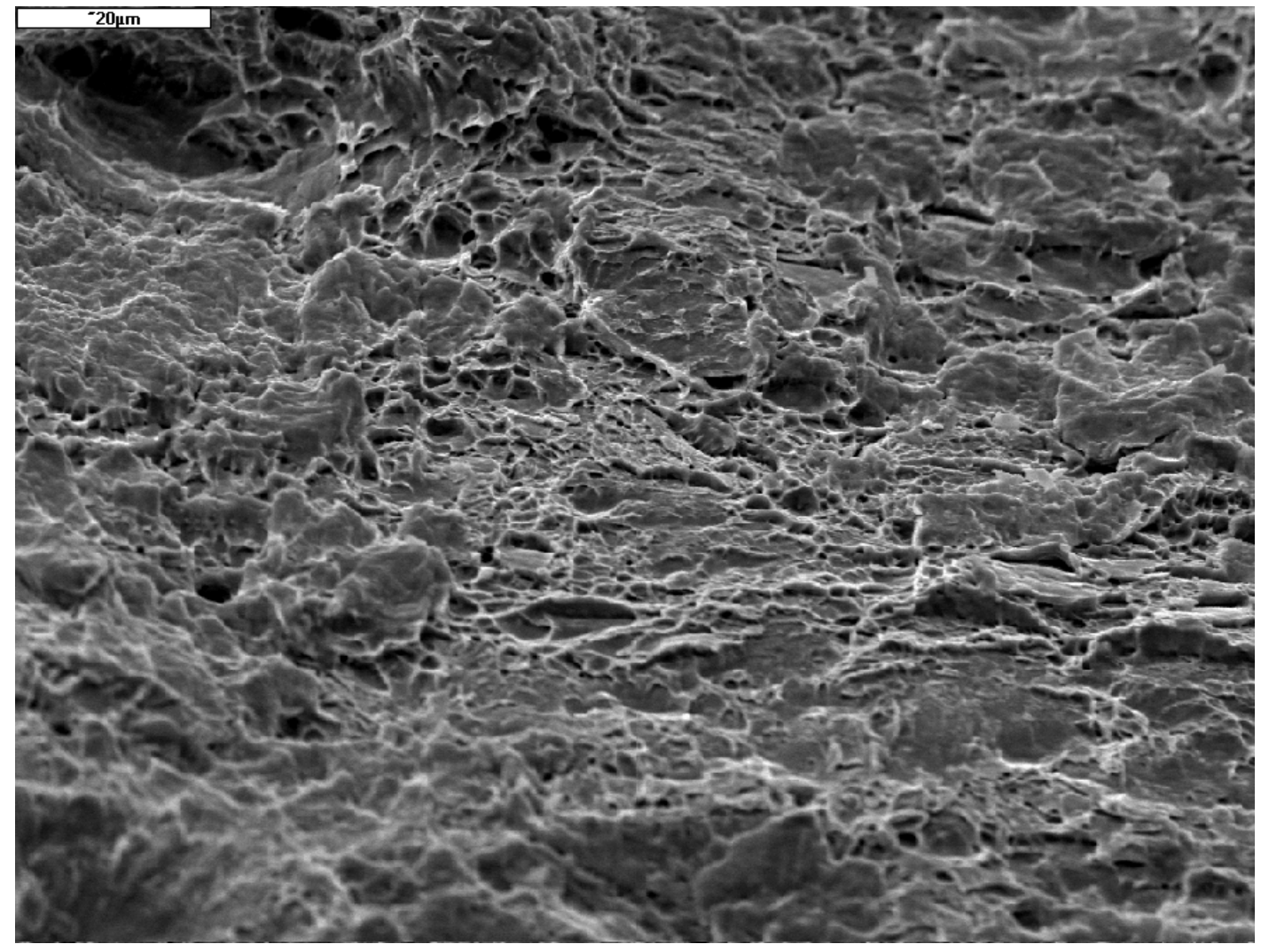

Figura 5.10. Fractura dúctil en la zona de inicio de propagación de la fisura (1000X).

A pesar de que el inicio de la propagación de la fisura ya se ha producido, si observamos la sección correspondiente al ensayo interrumpido 04 (Figura 5.12) se puede ver como la fisura ha propagado rápidamente desde el ensayo anterior, haciéndose pasante a lo largo de todo el espesor y provocando la creación de dos frentes de fisura. Estos frentes conforme vaya aumentando la carga, propagarán en sentidos opuestos hacia la zona de empotramiento de la probeta. Uno de estos frentes de fisura, el que se encuentra dentro del círculo en la Figura 5.12, se puede observar con detalle en la Figura 5.13, en ella se distingue fácilmente las zonas de rotura dúctil y frágil, y la zona de la fisura inicial.

Mediante el análisis SEM se ha comprobado que el punto de inicio de propagación de fisura se ha producido para una carga ligeramente inferior a la correspondiente al ensayo interrumpido $03(3.8 \mathrm{kN})$. También se ha podido observar que una vez que se inicia la fisura, ésta propaga muy rápidamente, por lo que el inicio de la fisura estará muy cercano al punto 03. En consecuencia, se ha considerado un valor de inicio de propagación de $P_{a p l}$ de $3.7 \mathrm{kN}$, ligeramente inferior al valor del ensayo interrumpido 03. 


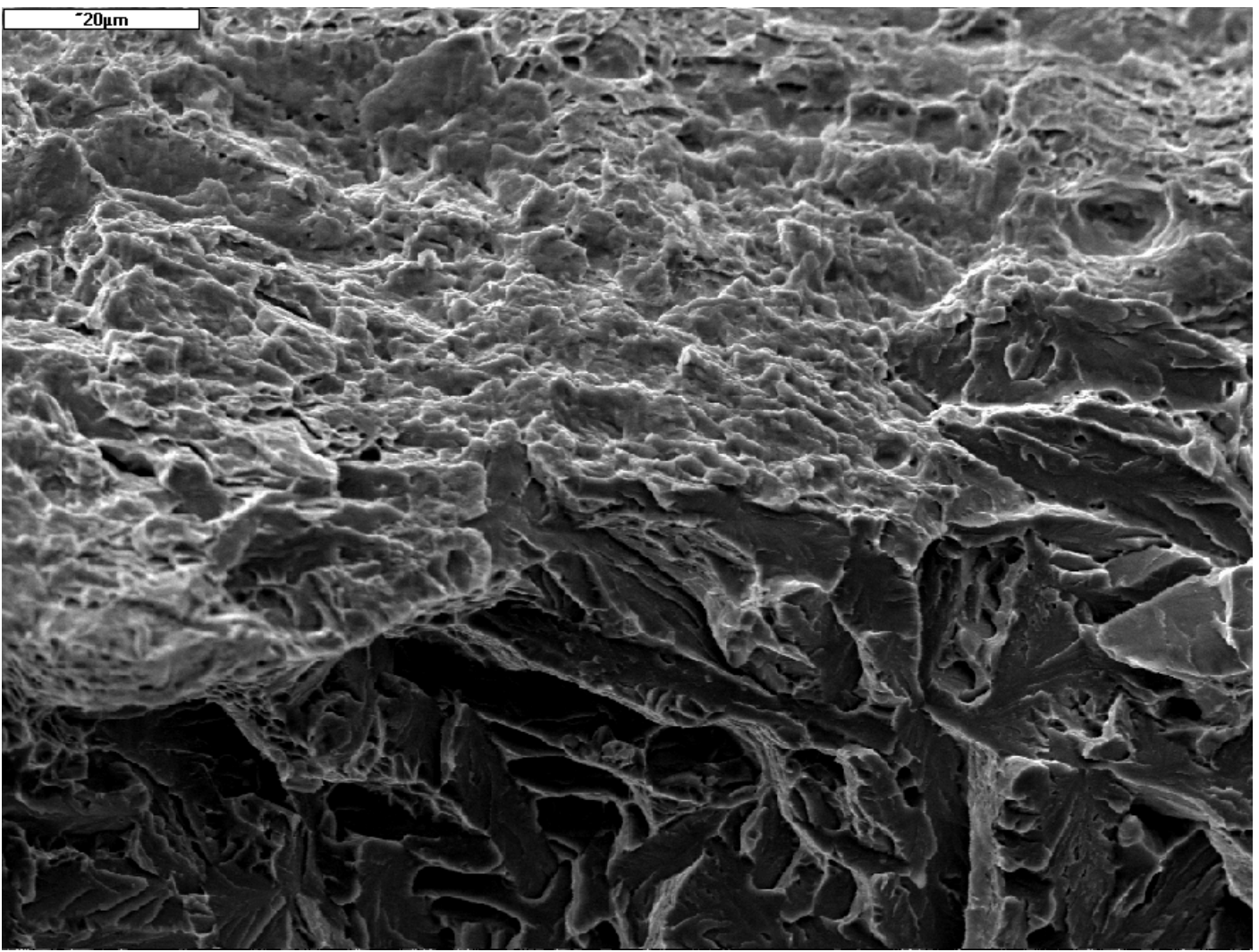

Figura 5.11. Zona de transición de fractura dúctil a frágil (1000X).

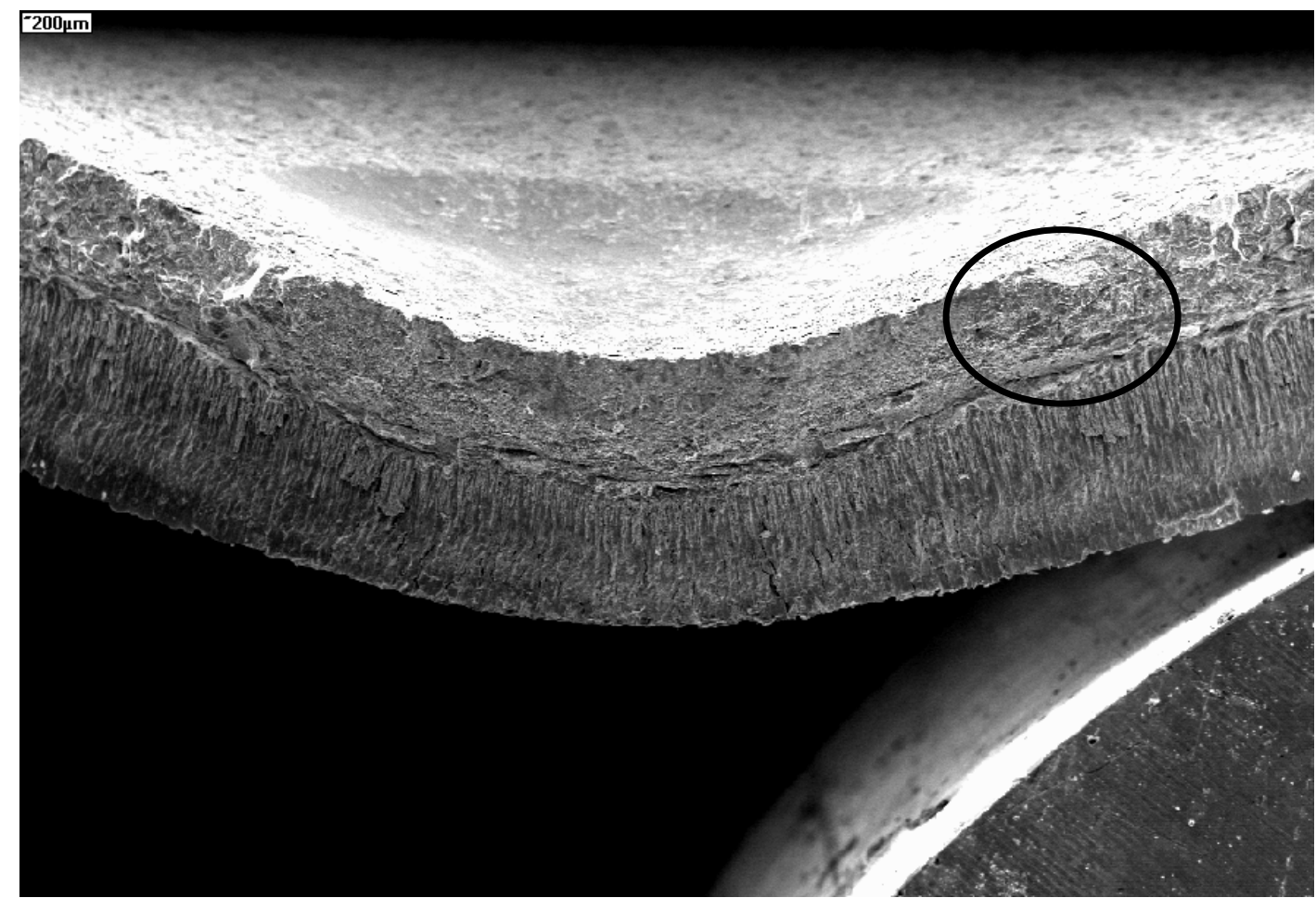

Figura 5.12. Zona central de la fisura en la probeta SPT0.5-04 (35X). 


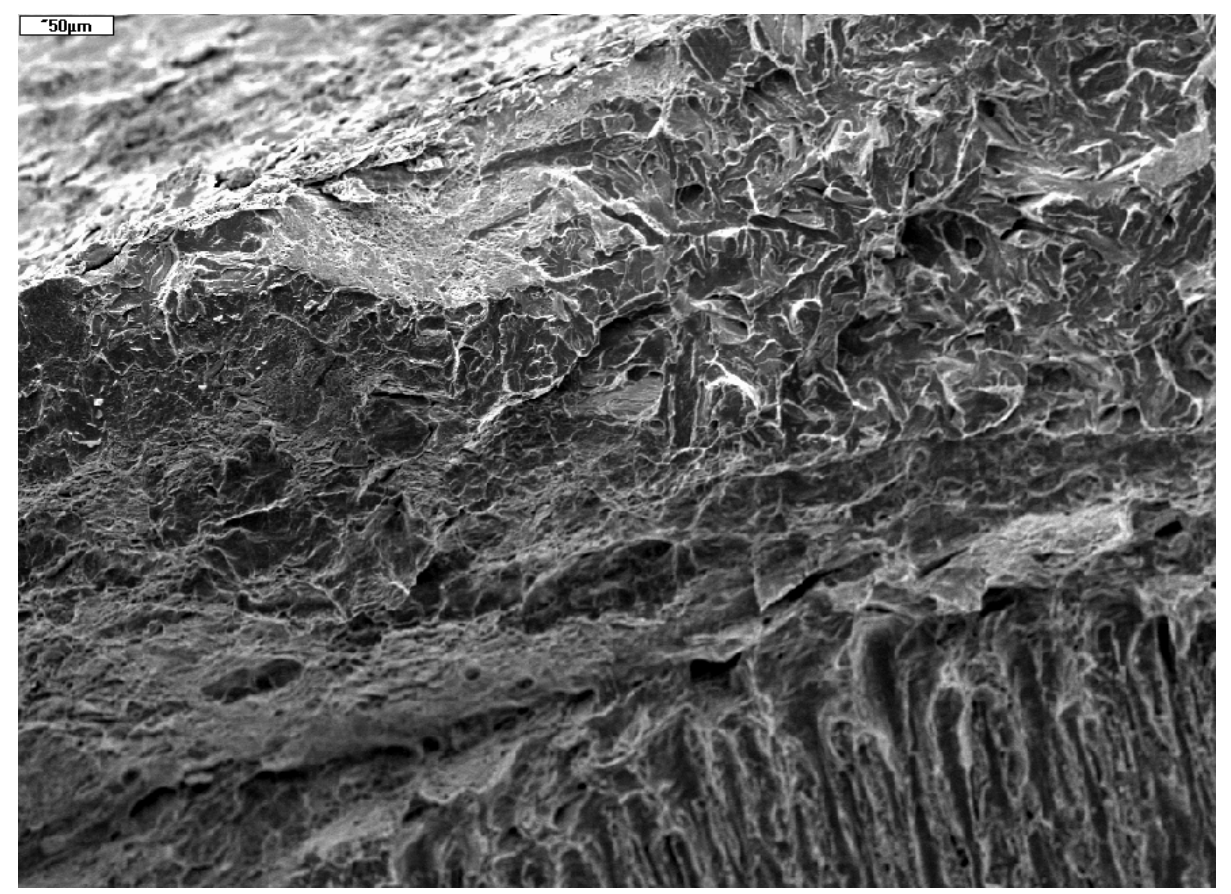

Figura 5.13. Frente de fisura en la probeta SPT0.5-04 (200X).

\subsubsection{Ensayos interrumpidos sobre probetas SPT prefisuradas con relación a/t de 0.4}

Una vez analizada la primera batería de ensayos SPT interrumpidos sobre una profundidad de fisura de $0.54 \mathrm{~mm}$, se ha realizado una segunda sobre probetas con una relación $a / t$ teórica de 0.4. Para no tener que ejecutar ensayos innecesarios se han efectuado dos ensayos completos con las probetas SPT0.4-01 y SPT0.4-02, para disponer de una curva completa que sirva de base para determinar la estrategia a seguir con los ensayos interrumpidos.

Se ha comprobado que la forma de la curva obtenida es la correspondiente a una relación $a / t<0.5$, por lo que se puede identificar el instante en el que la fisura se hace pasante, cuando se produce el descenso brusco de la capacidad resistente. Determinado este punto en la curva se ha realizado un ensayo interrumpido (probeta SPT0.4-03) al 90\% de la carga máxima. Las curvas carga-desplazamiento obtenidas se pueden ver en la Figura 5.14, donde se puede observar la buena repetibilidad de las primeras regiones para todas las curvas. Tras realizar el ensayo interrumpido se rompe de forma frágil la probeta y se realiza el análisis fractográfico de la misma, de forma análoga a la recogida en el apartado anterior. La Figura 5.15 muestra la superficie de fractura de la probeta, en la que se puede observar de nuevo tres 
zonas diferenciadas, correspondientes a la fisura inicial, a la propagación de fisura de forma dúctil y a la fractura frágil final para su observación y análisis. En este caso, de nuevo se puede concluir que el punto de inicio ha sido bien establecido dado que se ha alcanzado un nivel de carga en el ensayo interrumpido $(P=6.9 \mathrm{kN})$ ligeramente superior al que produce el inicio de la rotura, pudiendo de esta forma proceder a su observación. Este valor es evidentemente, un poco superior al del inicio, y en el apartado siguiente se ha aplicado el método de evaluación de la pendiente de la curva a esta probeta (probeta SPT0.4-03), para determinar con más exactitud el punto de inicio.

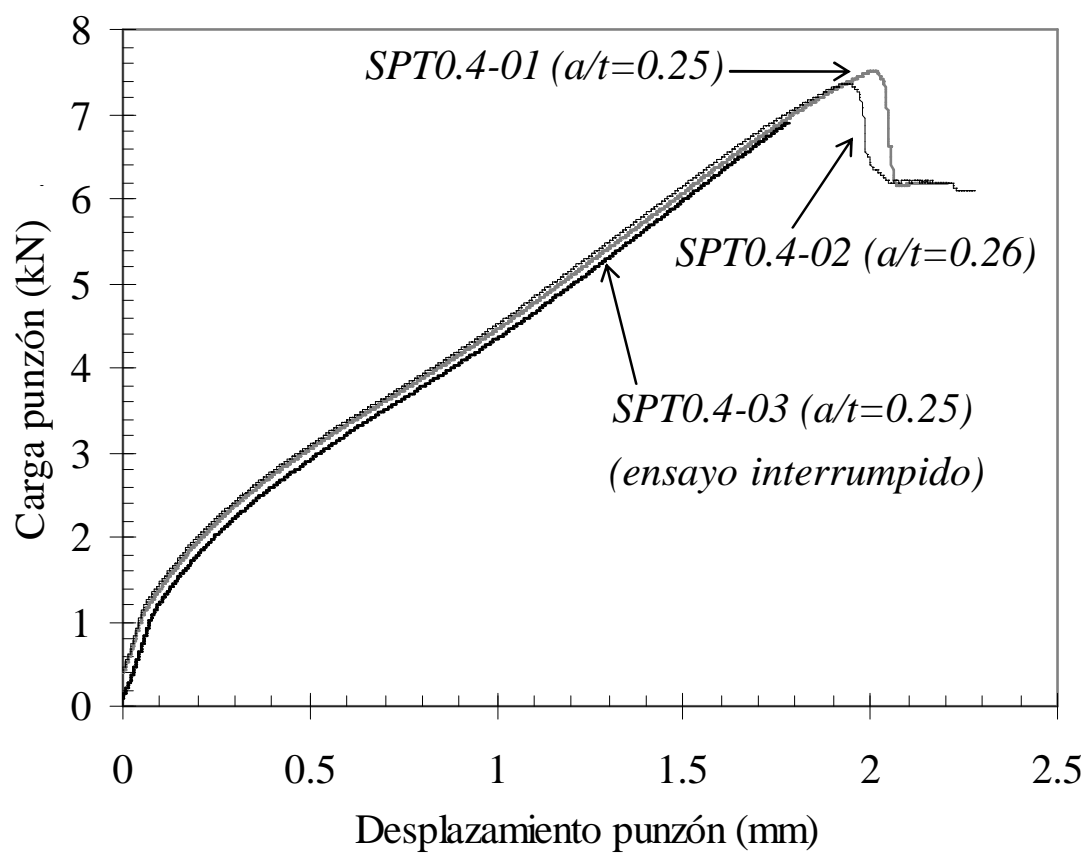

Figura 5.14. Curvas carga-desplazamiento del punzón obtenidas en los ensayos interrumpidos SPT sobre probetas con relación al t teórica de 0.4.

En consecuencia, podríamos decir que este método de ensayos interrumpidos presenta el interés de poder analizar los mecanismos de rotura y la evolución real de la fisura. Presenta por lo tanto, un elevado interés científico. Sin embargo, desde un punto de vista práctico se deben buscar otros métodos que permitan determinar el inicio de fisuración, sin tener que recurrir a ensayos interrumpidos que no permiten determinarlo con exactitud. En ese sentido, se ha procedido a analizar la forma de la curva carga-desplazamiento y a efectuar un ensayo con descargas elásticas, que permitan localizar de manera cómoda y fiable el punto de inicio de propagación de fisura. 


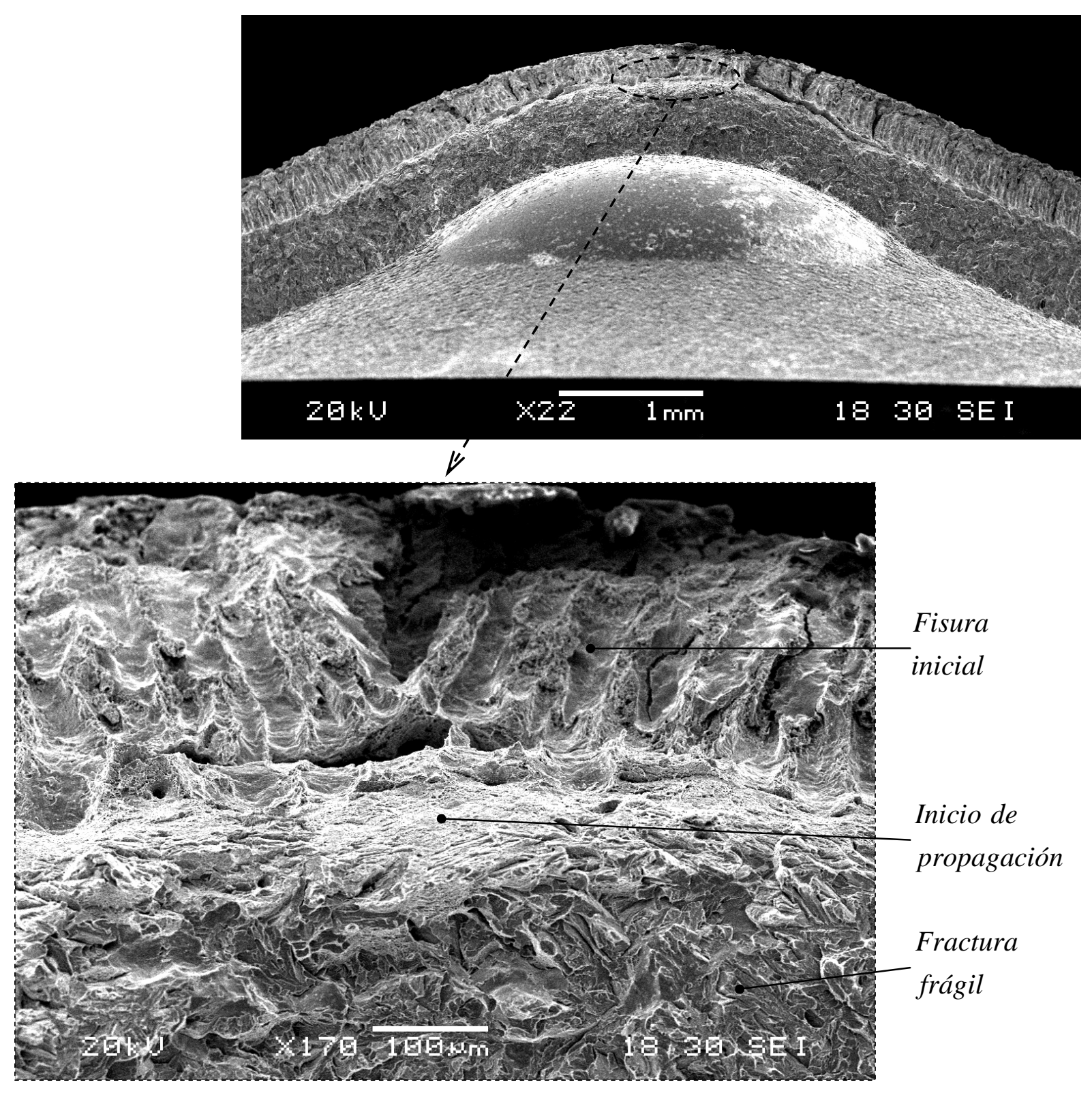

Figura 5.15. Superficie de fractura de la probeta SPT0.4-03.

\subsubsection{Método de evaluación de la pendiente de la curva}

Como se ha comentado en el Capítulo 3, este método consiste en analizar la variación de la pendiente en la curva carga-desplazamiento a lo largo de la región III para probetas SPT prefisuradas. Su aplicación a probetas con una relación $a / t>0.5$ no es aconsejable dado que el inicio de la fisuración se produce para cargas relativamente bajas, en el entorno del punto de inflexión existente entre la región II y III de la curva carga-desplazamiento, no pudiéndose 
ajustar este tramo de la curva a una recta. Este método ha sido aplicado a una batería de probetas SPT con una relación $a / t$ entre 0.2 y 0.4 , referenciadas como SPT0.4-01, SPT0.402, SPT0.4-03, SPT0.3-01, SPT0.3-02, SPT0.3-03, SPT0.2-01 у SPT0.2-02.

Las curvas carga-desplazamiento obtenidas en el ensayo hasta la rotura de cada una de estas probetas se puede observar en la Figura 5.16, donde de nuevo se aprecia la buena repetibilidad de las primeras regiones de las curvas. La longitud de fisura real de cada probeta ensayada aparece reflejada posteriormente en la Tabla 5.3, y puede observarse también en la propia Figura 5.16 la relación $a / t$ real. Se observa claramente que cuanto mayor es la fisura inicial (o la relación $a / t$ ) menor es la carga de rotura, lo cuál es completamente lógico.

La Figura 5.17 muestra la aplicación de este método a cada una de las probetas utilizadas, obteniendo en cada caso, el valor del desplazamiento del punzón para el que se produce el descenso de la pendiente de la curva $\left(\Delta p_{a p l}\right)$, lo cuál implica el inicio de propagación de fisura. La Tabla 5.1 recoge además de estos resultados, los valores de carga máxima $\left(P_{\max }\right)$ y carga en el inicio de propagación de fisura $\left(P_{a p l}\right)$, así como el ratio entre estos dos valores de carga.

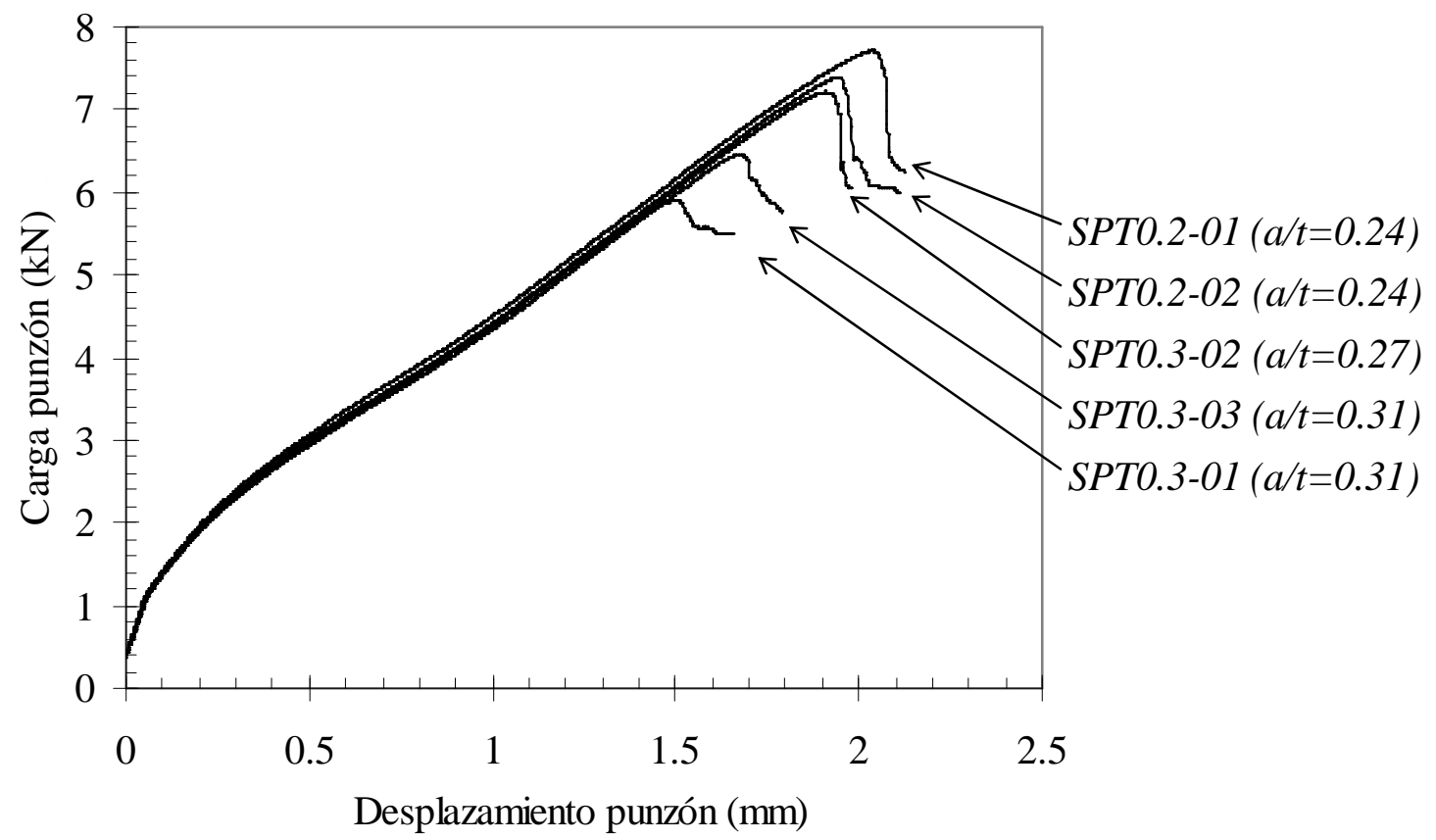

Figura 5.16. Curvas carga-desplazamiento del punzón obtenidas en los ensayos interrumpidos SPT sobre probetas con relación a/ $t$ teórica de 0.3 y 0.2. 

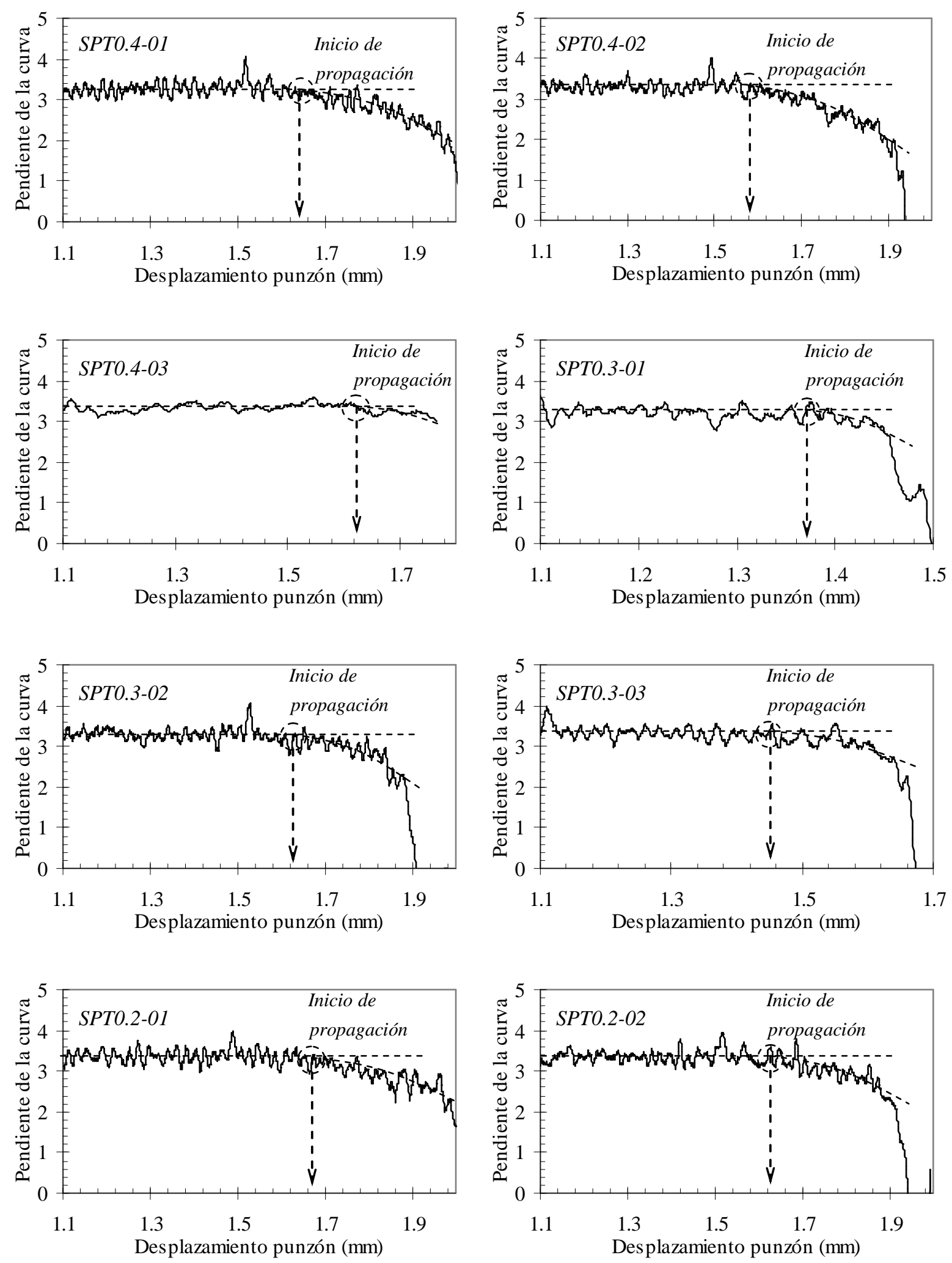

Figura 5.17. Evaluación de la pendiente de las curvas carga-desplazamiento del punzón de la Figura 5.14 y de la Figura 5.16. 
Tabla 5.1. Resultados del método de evaluación de la pendiente de la curva

\begin{tabular}{|c|c|c|c|c|}
\hline Probeta & $\Delta \boldsymbol{p}_{\text {apl }}(\mathbf{m m})$ & $\boldsymbol{P}_{a p l}(\boldsymbol{k N})$ & $\boldsymbol{P}_{\max }(\boldsymbol{k N})$ & $\begin{array}{c}\text { Ratio } \\
\boldsymbol{P}_{a p l} / \boldsymbol{P}_{\max }\end{array}$ \\
\hline SPT0.4-01 & 1.65 & 6.57 & 7.51 & 0.87 \\
\hline SPT0.4-02 & 1.58 & 6.42 & 7.37 & 0.87 \\
\hline SPT0.4-03 & 1.63 & 6.42 & - & - \\
\hline SPT0.3-01 & 1.37 & 5.59 & 5.91 & 0.95 \\
\hline SPT0.3-02 & 1.63 & 6.47 & 7.22 & 0.90 \\
\hline SPT0.3-03 & 1.45 & 5.82 & 6.47 & 0.90 \\
\hline SPT0.2-01 & 1.67 & 6.73 & 7.71 & 0.87 \\
\hline SPT0.2-02 & 1.63 & 6.52 & 7.39 & 0.88 \\
\hline
\end{tabular}

\subsubsection{Método de la flexibilidad}

Este método es el último de los tres métodos presentados en esta Tesis para la evaluación de la carga en el inicio de propagación de fisura, y a tenor de los resultados obtenidos, parece ser el más recomendable para su aplicación desde un punto de vista ingenieril. Se han empleado dos probetas SPT prefisuradas, referenciadas como SPT0.4-04 y SPT0.4-05, cuya longitud real de fisura medida después del ensayo resultó ser de 0.27 y $0.26 \mathrm{~mm}$ respectivamente. La Figura 5.18 muestra la curva carga-desplazamiento obtenida para cada una de estas probetas, en ella se han calculado las pendientes de cada una de las descargas, tal y como ha quedado reflejado en el Capítulo 3, las cuáles se han representado frente al desplazamiento del punzón en esa misma figura. La Figura 5.19 muestra el detalle del momento en el que la curva pendiente-desplazamiento pierde la linealidad, instante que se considera como inicio de propagación de fisura. Los valores de carga y desplazamiento del punzón de dicho instante se recogen en la Tabla 5.2, además del valor de carga máxima y del ratio $P_{a p l} / P_{\max }$ correspondiente a cada probeta. Destacar la buena repetibilidad de este ensayo, aplicado en este caso a dos probetas con idéntica relación $a / t$. En el gráfico de la Figura 5.18 también se ha evaluado y representado el valor de la pendiente inicial (con una precarga hasta $1 \mathrm{kN}$ ), comprobando la tendencia lineal hasta el inicio de propagación. 

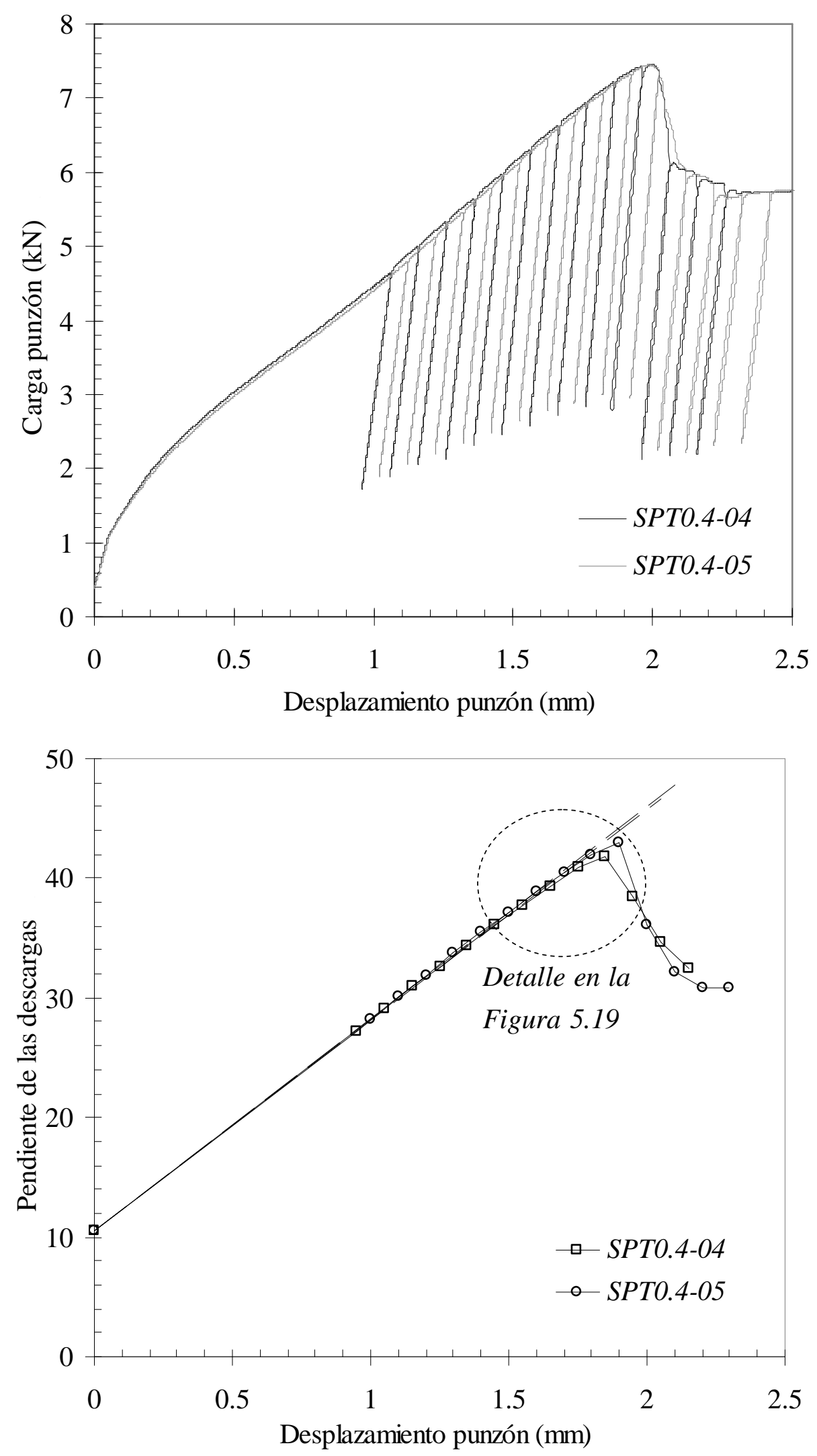

Figura 5.18. Método de la flexibilidad sobre probetas SPT con relación a / t teórica de 0.4. 

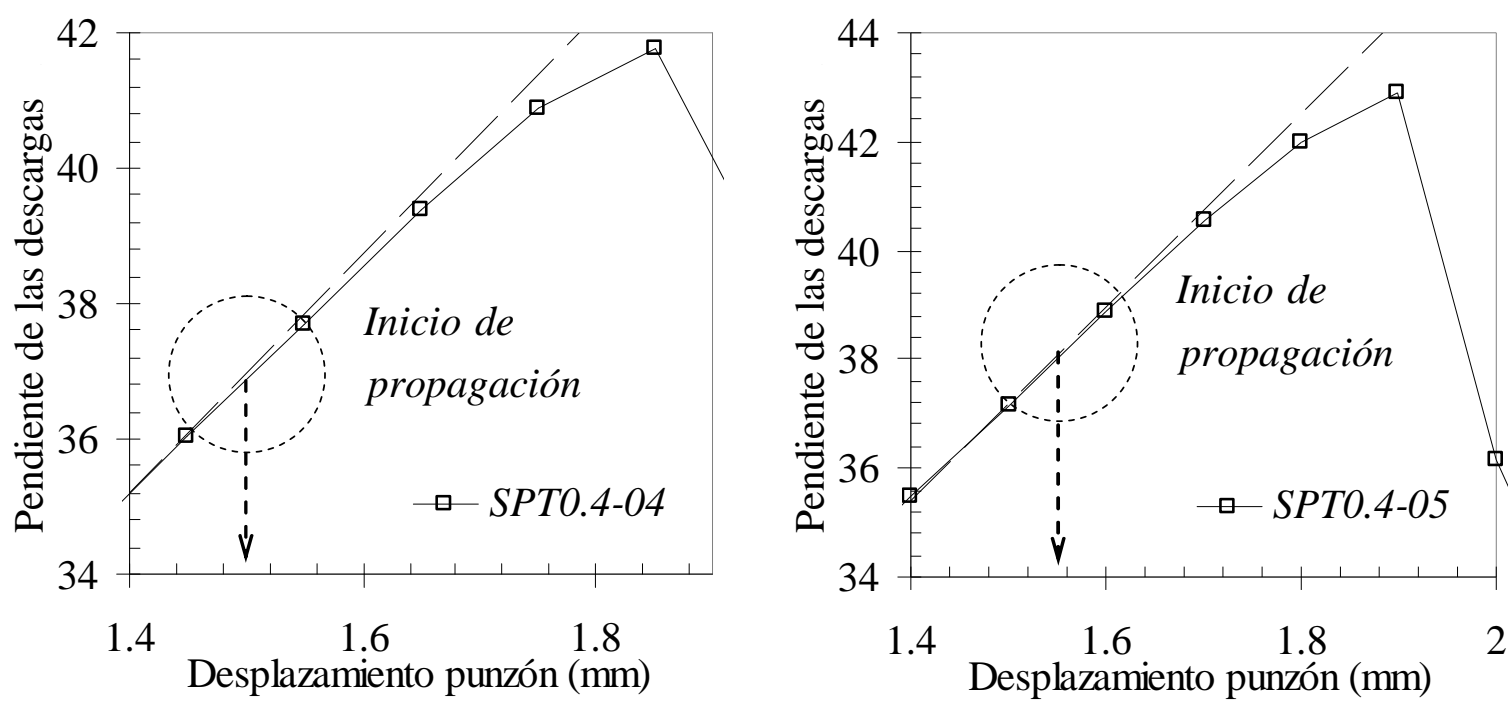

Figura 5.19. Detalle de las curvas pendiente-desplazamiento de la Figura 5.18.

Tabla 5.2. Resultados del método de la flexibilidad.

\begin{tabular}{|c|c|c|c|c|}
\hline Probeta & $\Delta \boldsymbol{p}_{\text {apl }}(\mathbf{m m})$ & $\boldsymbol{P}_{a p l}(\boldsymbol{k N})$ & $\boldsymbol{P}_{\max }(\boldsymbol{k N})$ & $\begin{array}{c}\text { Ratio } \\
\boldsymbol{P}_{a p l} / \boldsymbol{P}_{\max }\end{array}$ \\
\hline SPT0.4-04 & 1.5 & 6.12 & 7.46 & 0.82 \\
\hline SPT0.4-05 & 1.55 & 6.25 & 7.45 & 0.84 \\
\hline
\end{tabular}

\subsubsection{Profundidad de fisura de las probetas SPT prefisuradas}

A lo largo de la presente Tesis, se ha hecho especial hincapié en la necesidad de comprobar la profundidad real de la fisura ( $a$ ) de cada una de las probetas SPT utilizadas, ya que la técnica de microfisuración por láser deja una serie de irregularidades en el frente de fisura además, de la dificultad de conseguir el fondo de fisura deseado. Para ello se ha seguido el esquema de la Figura 5.20 en cada una de las probetas, en el que a partir de una fractografía de la sección de la fisura se ha establecido la relación $a / t$, a través de la determinación de las áreas correspondientes a la fisura inicial y al ligamento resistente.

Por otro lado, el espesor real $(t)$ de las probetas se ha medido con un micrómetro, obteniendo una alta repetibilidad en las medidas, por lo que se ha establecido el valor de $1 \mathrm{~mm}$ como 
espesor nominal para todas las probetas SPT ensayadas. Este hecho implica que en esta Tesis el valor numérico de la profundidad de fisura y de la relación $a / t$ son coincidentes. Estos valores quedan recogidos en la Tabla 5.3 para cada una de las probetas empleadas en los apartados anteriores. En el caso de las probetas con relación $a / t$ teórica de 0.5 , se propone un único valor de $a / t$ proveniente de la media de las cinco probetas, ya que con ellas se ha estimado un único valor de $P_{a p l}$. Tanto los valores de $P_{a p l}$, como los de $a / t$ serán imprescindibles en los apartados siguientes para determinar, en cada una de las probetas, los valores de $P_{U}, L_{r}$ y $K_{I}$.
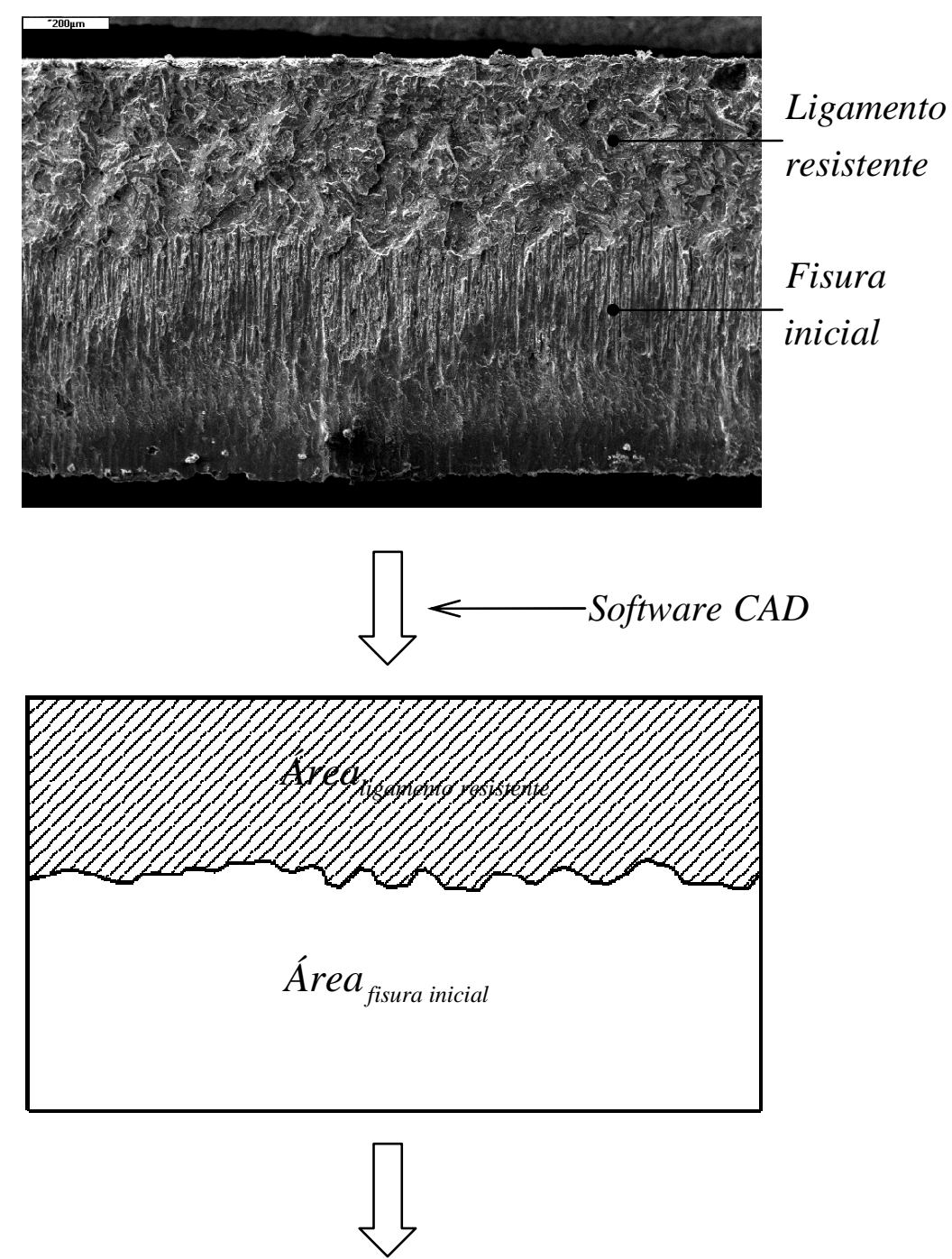

$$
a / t=\frac{\text {Área }_{\text {fisura inicial }}}{\text { Área } a_{\text {fisura inicial }}+\text { Área } a_{\text {ligamento resistente }}}
$$

Figura 5.20. Esquema para la determinación de la relación a/ $t$. 
Tabla 5.3. Profundidad de fisura y relación a/ $t$ de las probetas SPT ensayadas.

\begin{tabular}{|c|c|c|}
\hline Probeta & $\boldsymbol{a}(\mathbf{m m})$ & $\boldsymbol{a} / \boldsymbol{t}$ \\
\hline SPT0.5 & 0.54 & 0.54 \\
\hline SPT0.4-01 & 0.25 & 0.25 \\
\hline SPT0.4-02 & 0.26 & 0.26 \\
\hline SPT0.4-03 & 0.25 & 0.25 \\
\hline SPT0.4-04 & 0.27 & 0.27 \\
\hline SPT0.4-05 & 0.26 & 0.26 \\
\hline SPT0.3-01 & 0.31 & 0.31 \\
\hline SPT0.3-02 & 0.27 & 0.27 \\
\hline SPT0.3-03 & 0.31 & 0.31 \\
\hline SPT0.2-01 & 0.24 & 0.24 \\
\hline SPT0.2-02 & 0.24 & 0.24 \\
\hline
\end{tabular}

\subsection{OBTENCIÓN DE $P_{U}$}

Para la evaluación de la carga de colapso plástico $P_{U}$ se ha utilizado la superficie de respuesta determinada en el Capítulo 3, que contempla el efecto de las propiedades elastoplásticas del material y de la geometría de la fisura. La dimensión de probeta SPT utilizada ha sido 20x20x1 $\mathrm{mm}$. Se han obtenido dos soluciones de la carga de colapso plástico, una para las propiedades obtenidas de ensayos de tracción y otra para los valores estimados con un ensayo SPT convencional, referidas como $P_{U \text { trac }}$ y $P_{U S P T}$ respectivamente. Más adelante se analizarán y compararán los resultados alcanzados con cada una de ellas.

A partir de la función $g\left(a / t^{*}, \sigma_{0.2}^{*}, n^{*}\right)$, definida por la expresión (3.12), es posible determinar el valor de $P_{U}$ para cualquier combinación de valores de los parámetros $a / t$ (Tabla 5.3), $\sigma_{0.2}$ (Tabla 4.5) y $n$ (Tabla 4.5), previa codificación, siempre y cuando nos encontremos dentro del dominio esférico definido anteriormente para este diseño. La Tabla 5.4 muestra un 
ejemplo de aplicación de la hoja Excel desarrollada para tal fin, en la que tendríamos como variables de entrada (en negrita) los valores reales correspondientes a cada probeta de $a / t$, $\sigma_{0.2}$ y $n$ y como variables de salida (en negrita y cursiva) el valor de $P_{U}$ de la probeta utilizada y la distancia al centro del dominio del diseño, la cuál tiene que ser necesariamente menor a 1.682 para que la estimación de $P_{U}$ a través de este procedimiento se considere válida.

Para todas las probetas SPT empleadas la distancia al centro del dominio del diseño es menor que el máximo permitido, por lo que los valores obtenidos de $P_{U}$ para cada probeta pueden ser considerados válidos.

Tabla 5.4. Tabla Excel desarrollada para determinar el valor de $P_{U}$ en la probeta de 20x20x1mm a partir de la función $g\left(a / t^{*}, \sigma_{0.2}^{*}, n^{*}\right)$ definida por la expresión (3.12).

\begin{tabular}{|c|c|}
\hline Coeficiente & Valor \\
\hline$b_{0}$ & 3.664 \\
\hline$b_{1}$ & -1.132 \\
\hline$b_{2}$ & 1.993 \\
\hline$b_{3}$ & -0.243 \\
\hline$b_{11}$ & 0.130 \\
\hline$b_{22}$ & 0.065 \\
\hline$b_{33}$ & 0.168 \\
\hline$b_{12}$ & -0.797 \\
\hline$b_{13}$ & 0.068 \\
\hline$b_{23}$ & -0.077 \\
\hline
\end{tabular}

\begin{tabular}{|l|c|c|c|}
\cline { 2 - 4 } \multicolumn{1}{c|}{} & $a / t$ & $\sigma_{0.2}(M P a)$ & $n$ \\
\hline Límite inferior & 0.1 & 200 & 4 \\
\hline Límite superior & 0.7 & 1400 & 50 \\
\hline Centro del dominio & 0.4 & 800 & 27 \\
\hline
\end{tabular}

\begin{tabular}{|l|c|c|c|c|}
\cline { 2 - 4 } \multicolumn{1}{c|}{} & \multicolumn{3}{c}{} & $P_{U}(k N)$ \\
\cline { 2 - 4 } \multicolumn{1}{c|}{} & $a / t$ & $\sigma_{0.2}$ & $n$ & $\mathbf{3 . 9 6}$ \\
\hline Valores reales & $\mathbf{0 . 5 4}$ & $\mathbf{1 1 0 0}$ & $\mathbf{3 8 . 8}$ & \multicolumn{1}{c}{} \\
\hline Valores codificados & 0.78 & 0.84 & 0.86 & \\
\hline
\end{tabular}

La Tabla 5.5 recoge los valores de $P_{U}$ para todos los ensayos efectuados, así como el valor de la carga aplicada en el inicio de propagación $P_{a p l}$ y el método utilizado para su evaluación. Estos valores se utilizarán en el apartado siguiente para determinar el valor del ratio de carga $L_{r}$ correspondiente a cada probeta. 
Tabla 5.5. Resultados de $P_{U}$ según la alternativa utilizada para determinar las propiedades elastoplásticas del material.

\begin{tabular}{|c|c|c|c|c|c|}
\hline Probeta & $\begin{array}{c}\boldsymbol{P}_{\text {apl }} \\
(\boldsymbol{k N})\end{array}$ & Método evaluación $\boldsymbol{P}_{\text {apl }}$ & $\boldsymbol{a} / \boldsymbol{t}$ & $\begin{array}{c}\boldsymbol{P}_{\boldsymbol{U} \text { trac }} \\
(\boldsymbol{k N})\end{array}$ & $\begin{array}{c}\boldsymbol{P}_{\boldsymbol{U} \text { SPT }} \\
(\boldsymbol{k N})\end{array}$ \\
\hline SPT0.5 & 3.7 & Ensayo interrumpido & 0.54 & 3.96 & 3.79 \\
\hline SPT0.4-01 & 6.57 & Pendiente de la curva & 0.25 & 6.8 & 6.51 \\
\hline SPT0.4-02 & 6.42 & Pendiente de la curva & 0.26 & 6.69 & 6.41 \\
\hline SPT0.4-03 & 6.42 & E. interrumpido / P. de la curva & 0.25 & 6.8 & 6.51 \\
\hline SPT0.4-04 & 6.12 & Método de la flexibilidad & 0.27 & 6.59 & 6.3 \\
\hline SPT0.4-05 & 6.25 & Método de la flexibilidad & 0.26 & 6.69 & 6.41 \\
\hline SPT0.3-01 & 5.59 & Pendiente de la curva & 0.31 & 6.16 & 5.89 \\
\hline SPT0.3-02 & 6.47 & Pendiente de la curva & 0.27 & 6.59 & 6.3 \\
\hline SPT0.3-03 & 5.82 & Pendiente de la curva & 0.31 & 6.16 & 5.89 \\
\hline SPT0.2-01 & 6.73 & Pendiente de la curva & 0.24 & 6.91 & 6.62 \\
\hline SPT0.2-02 & 6.52 & Pendiente de la curva & 0.24 & 6.91 & 6.62 \\
\hline
\end{tabular}

\subsection{CÁLCULO DEL RATIO DE CARGA, $L_{r}$}

Hasta el momento se ha establecido para cada probeta, dos valores de la carga de colapso $P_{U}$ en función de la alternativa utilizada para determinar las propiedades elastoplásticas del material, y un valor para la carga aplicada sobre la probeta en el instante de inicio de propagación de fisura $P_{a p l}$. Aplicando estos valores directamente en la expresión (2.11):

$L_{r}=\frac{P_{a p l}}{P_{U}}$

se obtienen dos resultados posibles del valor de $L_{r}$ para cada probeta, los cuáles quedan recogidos en la Tabla 5.6. Cada uno de estos valores de $L_{r}$ será el utilizado más adelante en la aplicación de la metodología desarrollada en la presente Tesis. 
Tabla 5.6. Resultados de $L_{r}$ en función de la alternativa utilizada para determinar las propiedades elastoplásticas del material.

\begin{tabular}{|c|c|c|c|c|c|}
\hline Probeta & $\begin{array}{c}\boldsymbol{P}_{a p l} \\
(\boldsymbol{k} \boldsymbol{N})\end{array}$ & $\begin{array}{c}\boldsymbol{P}_{\boldsymbol{U} \text { trac }} \\
(\boldsymbol{k} \boldsymbol{N})\end{array}$ & $\begin{array}{c}\boldsymbol{P}_{\boldsymbol{U} \text { SPT }} \\
(\boldsymbol{k} \boldsymbol{N})\end{array}$ & $\boldsymbol{L}_{\boldsymbol{r} \text { trac }}$ & $\boldsymbol{L}_{\boldsymbol{r} \text { SPT }}$ \\
\hline SPT0.5 & 3.7 & 3.96 & 3.79 & 0.93 & 0.98 \\
\hline SPT0.4-01 & 6.57 & 6.8 & 6.51 & 0.97 & 1.01 \\
\hline SPT0.4-02 & 6.42 & 6.69 & 6.41 & 0.96 & 1.00 \\
\hline SPT0.4-03 & 6.42 & 6.8 & 6.51 & 0.94 & 0.99 \\
\hline SPT0.4-04 & 6.12 & 6.59 & 6.3 & 0.93 & 0.97 \\
\hline SPT0.4-05 & 6.25 & 6.69 & 6.41 & 0.93 & 0.98 \\
\hline SPT0.3-01 & 5.59 & 6.16 & 5.89 & 0.91 & 0.95 \\
\hline SPT0.3-02 & 6.47 & 6.59 & 6.3 & 0.98 & 1.03 \\
\hline SPT0.3-03 & 5.82 & 6.16 & 5.89 & 0.94 & 0.99 \\
\hline SPT0.2-01 & 6.73 & 6.91 & 6.62 & 0.97 & 1.02 \\
\hline SPT0.2-02 & 6.52 & 6.91 & 6.62 & 0.94 & 0.98 \\
\hline
\end{tabular}

\subsection{REPRESENTACIÓN DEL DIAGRAMA DE FALLO, FAD}

Una vez obtenidos los valores de $L_{r}$ del apartado anterior, el siguiente paso, previo a la obtención de $K_{r}$, es la representación del diagrama FAD según la opción que se desee en función de los datos disponibles.

Cabe destacar que las curvas del diagrama de fallo según las Opciones 1 y 2 son independientes de la relación $a / t$ de cada probeta SPT utilizada, ya que las expresiones (2.14) у (2.15) que las definen respectivamente no tienen en cuenta dicha relación. En cambio, al aplicar la Opción 3 del FAD, a priori, no existe tal independencia, ya que habría que realizar una simulación numérica para cada relación $a / t$, para poder determinar los parámetros de la expresión (2.16) que define esta opción y de este modo representar la curva del diagrama de fallo. 


\subsubsection{Opción 1}

Para esta opción de FAD, como se ha visto anteriormente, utilizaremos las expresiones (2.13) y (2.14):

$$
\begin{array}{ll}
L_{r}^{\max }=\frac{1}{2} \cdot \frac{\left(\sigma_{0.2}+\sigma_{u t s}\right)}{\sigma_{0.2}} & \\
K_{r}=\left(1-0.14 \cdot L_{r}^{2}\right)\left[0.3+0.7 \cdot \exp \left(-0.65 \cdot L_{r}^{6}\right)\right] & \text { si } L_{r} \leq L_{r}^{\max } \\
K_{r}=0 & \text { si } \quad L_{r}>L_{r}^{\max }
\end{array}
$$

que son las que definen la forma del diagrama, donde sólo es necesario conocer los valores de $\sigma_{0.2}$ y $\sigma_{u t s}$ del acero 15-5 PH para determinar la línea de corte del diagrama. Para este acero estos dos valores han sido previamente obtenidos a través del ensayo de tracción uniaxial y también han sido estimados con el ensayo SPT convencional, obteniendo de este modo dos líneas de corte diferentes en el diagrama. La Tabla 5.7 recoge estos valores de $\sigma_{0.2}$ y $\sigma_{u t s}$, así como los valores de $L_{r}^{\max }$ correspondientes a las líneas de corte del diagrama. La Figura 5.21 muestra el diagrama FAD, para el acero 15-5 PH, siguiendo la Opción 1 de análisis. Dado que la estimación de los parámetros $\sigma_{0.2}$ y $\sigma_{u t s}$ a partir del ensayo SPT es bastante buena apenas existe diferencia entre ambas aproximaciones.

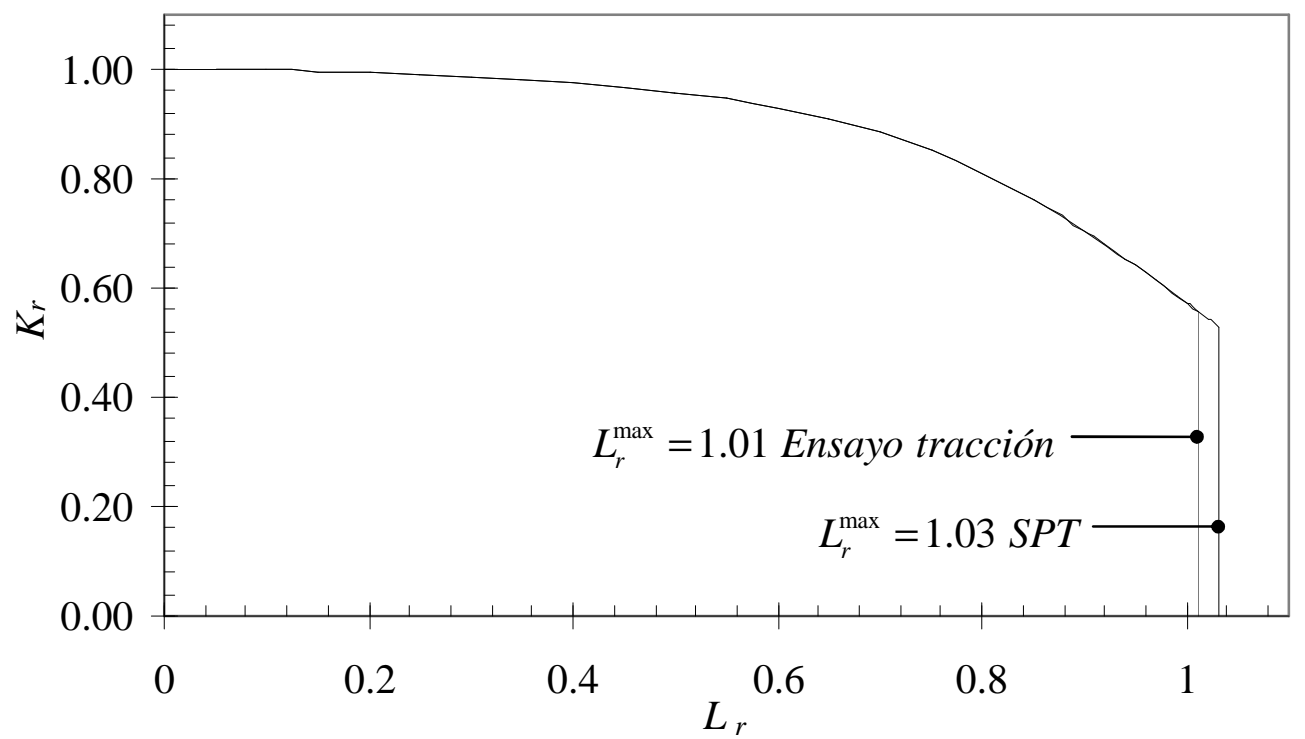

Figura 5.21. Diagrama FAD Opción 1 para el acero 15-5 PH. 
Tabla 5.7. Valores de $L_{r}^{\max }$.

\begin{tabular}{|c|c|c|c|}
\hline $\begin{array}{c}\text { Método de } \\
\text { obtención }\end{array}$ & $\boldsymbol{\sigma}_{0.2}(\mathbf{M P a})$ & $\boldsymbol{\sigma}_{u t s}(\mathbf{M P a})$ & $\boldsymbol{L}_{\boldsymbol{r}}^{\max }$ \\
\hline Ensayo tracción & 1100 & 1131 & 1.01 \\
\hline SPT & 1046.7 & 1111.9 & 1.03 \\
\hline
\end{tabular}

\subsubsection{Opción 2}

La condición necesaria para poder aplicar esta opción es conocer la curva completa tensióndeformación del material, la cuál se ha determinado a partir del ensayo de tracción uniaxial. La utilización única del ensayo SPT permite, como ha quedado establecido en el Capítulo 3, junto con una simulación numérica de dicho ensayo, estimar el valor de $\sigma_{y}, \sigma_{u t s}$ y $n$, pudiendo ajustar dicho comportamiento a una ecuación tipo Ramberg Osgood. Esta segunda aproximación permite representar el diagrama FAD de la ecuación Ramberg Osgood estimada con el SPT, como si se tratase del ensayo de tracción real. Independientemente de la alternativa y de la relación $a / t$ de cada probeta SPT utilizada, la Opción 2 queda definida por la expresión (2.15):

$$
\begin{array}{lll}
K_{r}=\left(\frac{E \cdot \varepsilon_{r e f}}{L_{r} \cdot \sigma_{0.2}}+\frac{L_{r}^{3} \cdot \sigma_{0.2}}{2 \cdot E \cdot \varepsilon_{r e f}}\right)^{-1 / 2} & \text { si } & L_{r} \leq L_{r}^{\max } \\
K_{r}=0 & \text { si } & L_{r}>L_{r}^{\max }
\end{array}
$$

donde los valores de la línea de corte del diagrama $L_{r}^{\max }$ recogidos en la Tabla 5.7 siguen siendo válidos para esta opción, utilizando en cada caso el valor correspondiente a la alternativa elegida. La Figura 5.22 muestra el diagrama FAD Opción 2 para el acero 15-5 PH obtenido a partir de los valores de $E, n, \sigma_{0.2}$ y $\sigma_{u}$ determinados mediante el ensayo de tracción y el obtenido a partir de los valores de $E, n, \sigma_{0.2}$ y $\sigma_{u}$ estimados con el ensayo SPT. Se puede apreciar en estas dos figuras como la forma de la curva que define el FAD es similar pero ligeramente diferente, ya que los valores de $E, n, \sigma_{0.2}$ y $\sigma_{u}$ reales y estimados son diferentes. 


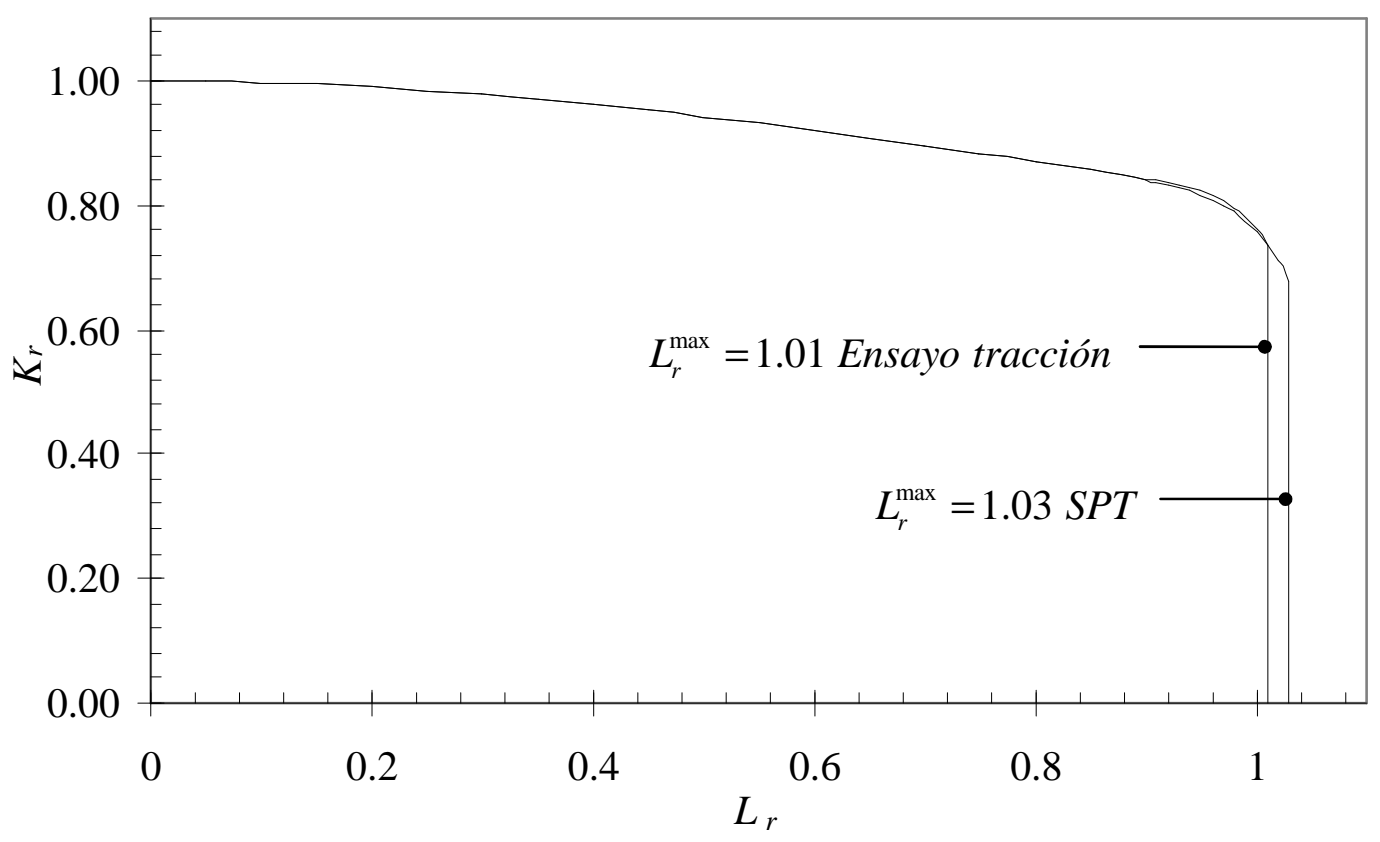

Figura 5.22. Diagrama FAD Opción 2 para el acero 15-5 PH.

\subsubsection{Opción 3}

La aplicación de la Opción 3, queda definida por la expresión (2.16):

$$
\begin{array}{lll}
K_{r}=\left(\frac{J_{e}}{J_{a p p}}\right)^{1 / 2} & \text { si } & L_{r} \leq L_{r}^{\max } \\
K_{r}=0 & \text { si } & L_{r}>L_{r}^{\max }
\end{array}
$$

En este caso es necesario llevar a cabo una simulación numérica para determinar los parámetros $J_{a p p}$ y $J_{e}$ de dicha expresión, siendo $J_{a p p}$ la integral $J$ aplicada y $J_{e}$ su parte elástica. A la hora de realizar dicha simulación se han contemplado, como en el resto de análisis, dos alternativas, en función de si los parámetros $E, n, \sigma_{0.2}$ y $\sigma_{u}$ utilizados son los determinados mediante el ensayo de tracción, o bien, son los estimados a partir del SPT convencional. Se ha comprobado que la elección de una u otra alternativa no influye notablemente en la curva del diagrama obtenida, dada la buena estimación de los parámetros elastoplásticos obtenida con el ensayo SPT. 
El parámetro que sí presenta mayor influencia en esta opción de análisis es la relación $a / t$. En este sentido, se ha efectuado una simulación para las relaciones $a / t$ de $0.25,0.4$ y 0.55 , interpolando posteriormente los resultados obtenidos para la longitud real de fisura de cada probeta SPT empleada.

La integral $J$ ha sido evaluada en el entorno de la punta de fisura teniendo en cuenta toda la longitud de fisura. Dado que es en la zona central de la probeta donde se producen los estados tensionales más elevados y donde se inicia la rotura, será en esta zona de donde se extraigan los valores de dicha integral. Una vez determinada la integral $J$, es posible obtener los parámetros $J_{a p p}$ y $J_{e}$ en función de la carga del punzón para la posterior representación del diagrama FAD según la Opción 3.

La Figura 5.23 muestra la evolución típica, en las probetas SPT prefisuradas, de los parámetros $J_{a p p}$ y $J_{e}$ frente a la carga del punzón. Una vez calculados los parámetros $J_{a p p} \mathrm{y}$ $J_{e}$ se ha representado el diagrama FAD Opción 3 definido por la expresión (2.16) para cada una de las tres relaciones $a / t$ simuladas. Los diagramas obtenidos son los que se pueden ver en la Figura 5.24.

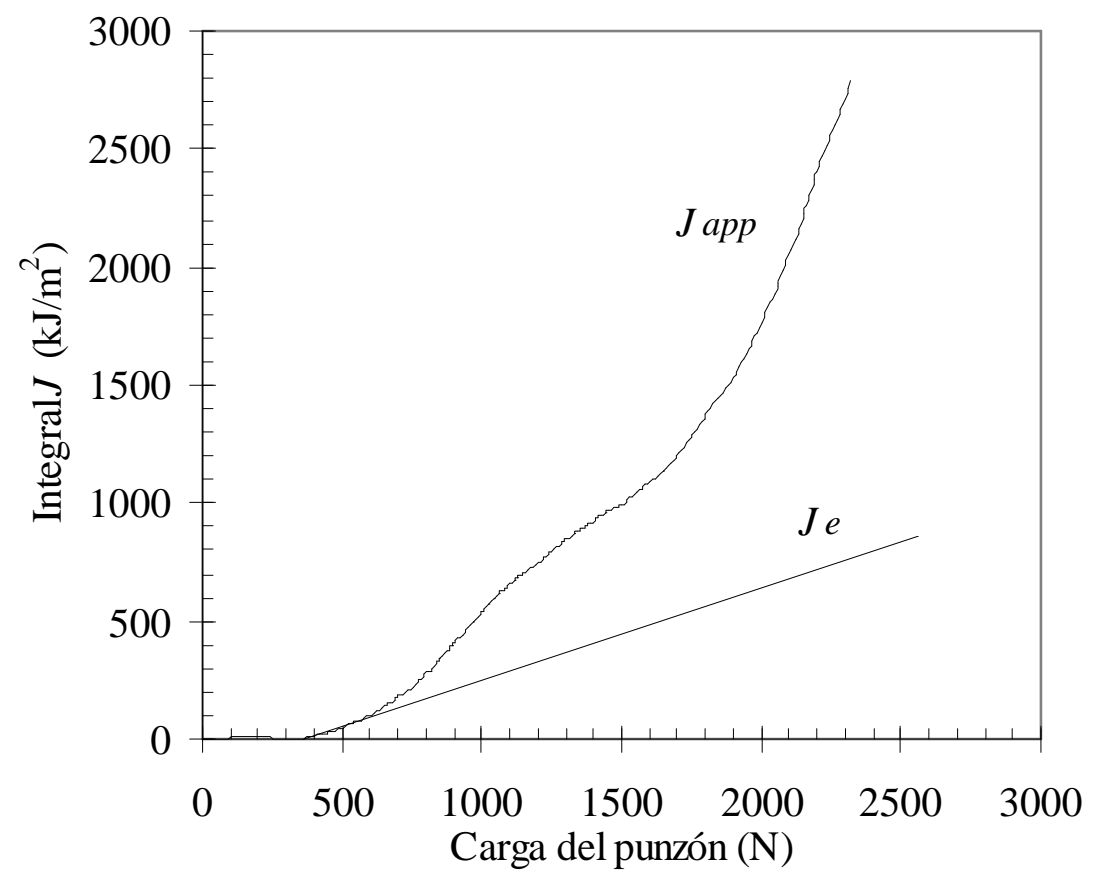

Figura 5.23. Evolución de los parámetros $J_{a p p}$ y $J_{e}$ en función de la carga del punzón. 


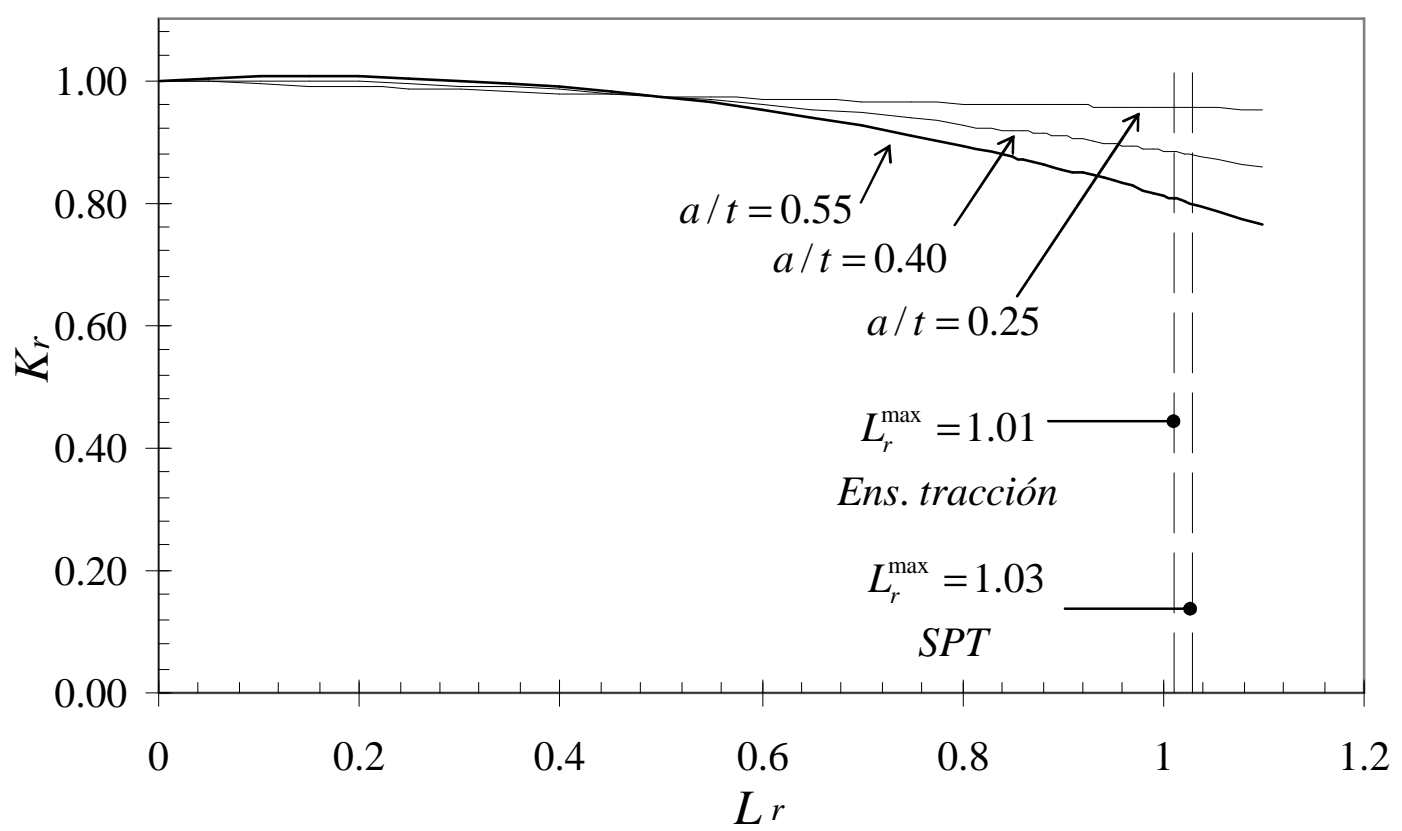

Figura 5.24. Diagramas FAD Opción 3 para el acero 15-5 PH a partir de la relación a / $t$.

\subsection{OBTENCIÓN DEL RATIO DE TENACIDAD, $K_{r}$}

Una vez representados los diagramas FAD del apartado anterior, resulta relativamente sencillo obtener el valor de los diferentes $K_{r}$, a partir de los correspondientes valores de $L_{r}$ determinados en el apartado 5.4, mediante el punto de corte de dicha abscisa con la curva del diagrama. Este punto del diagrama es considerado como el que define el instante en el que comienza a propagar la fisura en la probeta analizada, quedando definido por lo tanto el valor de la ordenada de dicho punto.

Todos los valores de $L_{r}$ determinados en el apartado 5.4 se encuentran dentro de la zona válida del eje de abscisas de los diagramas, ya que su valor es menor o igual que el valor correspondiente a la línea de corte $L_{r}^{\max }$ en función de la alternativa elegida. Dada la elevada ductilidad del material se observa sin embargo que los valores de $L_{r}$ están en el entorno de 0.9-1.0. La Tabla 5.8 recoge los valores del ratio de tenacidad $K_{r}$, determinados en función de la opción de FAD elegida y del valor de $L_{r}$ utilizado en cada una de las probetas SPT prefisuradas. 
Tabla 5.8. Resultados de $K_{r}$ según la opción de FAD elegida y del valor de $L_{r}$.

\begin{tabular}{|c|c|c|c|c|c|c|c|c|}
\hline \multirow[b]{2}{*}{ Probeta } & \multirow[b]{2}{*}{$L_{r \text { trac }}$} & \multirow[b]{2}{*}{$L_{r S P T}$} & \multicolumn{2}{|c|}{ FAD Opción 1} & \multicolumn{2}{|c|}{ FAD Opción 2} & \multicolumn{2}{|c|}{ FAD Opción 3} \\
\hline & & & $K_{r 1 \text { trac }}$ & $K_{r 1 S P T}$ & $K_{r 2 t r a c}$ & $K_{r 2 S P T}$ & $K_{r 3 t r a c}$ & $K_{r 3 S P T}$ \\
\hline SPT0.5 & 0.93 & 0.98 & 0.67 & 0.60 & 0.83 & 0.79 & 0.85 & 0.83 \\
\hline SPT0.4-01 & 0.97 & 1.01 & 0.61 & 0.56 & 0.81 & 0.74 & 0.96 & 0.96 \\
\hline SPT0.4-02 & 0.96 & 1.00 & 0.63 & 0.57 & 0.82 & 0.76 & 0.95 & 0.95 \\
\hline SPT0.4-03 & 0.94 & 0.99 & 0.65 & 0.59 & 0.83 & 0.77 & 0.96 & 0.96 \\
\hline SPT0.4-04 & 0.93 & 0.97 & 0.67 & 0.61 & 0.83 & 0.80 & 0.95 & 0.95 \\
\hline SPT0.4-05 & 0.93 & 0.98 & 0.67 & 0.60 & 0.83 & 0.79 & 0.95 & 0.95 \\
\hline SPT0.3-01 & 0.91 & 0.95 & 0.69 & 0.64 & 0.84 & 0.82 & 0.94 & 0.93 \\
\hline SPT0.3-02 & 0.98 & 1.03 & 0.60 & 0.53 & 0.80 & 0.68 & 0.95 & 0.94 \\
\hline SPT0.3-03 & 0.94 & 0.99 & 0.65 & 0.59 & 0.83 & 0.77 & 0.93 & 0.93 \\
\hline SPT0.2-01 & 0.97 & 1.02 & 0.61 & 0.54 & 0.81 & 0.71 & 0.96 & 0.96 \\
\hline SPT0.2-02 & 0.94 & 0.98 & 0.65 & 0.60 & 0.83 & 0.79 & 0.96 & 0.96 \\
\hline
\end{tabular}

\subsection{OBTENCIÓN DE $K_{I}$}

En el apartado 3.6 de la presente Tesis se ha deducido la expresión (3.17), que permite evaluar $K_{I}$ en función de la carga aplicada y de la relación $a / t$ real para las probetas SPT prefisuradas de dimensiones 20x20x1 $\mathrm{mm}$.

$K_{I 20 \times 20 \times 1}=\left[-138.81 \cdot\left(\frac{a}{t}\right)^{2}+96.81 \cdot\left(\frac{a}{t}\right)+18.9\right] \cdot P^{0.9399-\frac{0.0461}{\ln \left(\frac{a}{t}\right)}-\frac{0.0324}{\left[\ln \left(\frac{a}{t}\right)\right]^{2}}}$

Introduciendo en dicha ecuación los valores determinados anteriormente de la carga de inicio de fisuración $P_{a p l}$ (Tabla 5.5) y de la relación $a / t$ real de cada una de las probetas (Tabla 5.3) se obtienen los correspondientes valores de $K_{I}$ en el instante de propagación de fisura que se muestran en la Tabla 5.9. 
Tabla 5.9. Resultados de $K_{I}$ a partir de la expresión (3.17).

\begin{tabular}{|c|c|c|c|}
\hline Probeta & $\begin{array}{c}\boldsymbol{P}_{a p l} \\
(\boldsymbol{k} \boldsymbol{N})\end{array}$ & $\boldsymbol{a} / \boldsymbol{t}$ & $\begin{array}{c}\boldsymbol{K}_{\boldsymbol{I}} \\
\left(\boldsymbol{M P a} \cdot \boldsymbol{m}^{1 / 2}\right)\end{array}$ \\
\hline SPT0.5 & 3.7 & 0.54 & 104.3 \\
\hline SPT0.4-01 & 6.57 & 0.25 & 213.8 \\
\hline SPT0.4-02 & 6.42 & 0.26 & 209.8 \\
\hline SPT0.4-03 & 6.42 & 0.25 & 209.1 \\
\hline SPT0.4-04 & 6.12 & 0.27 & 200.9 \\
\hline SPT0.4-05 & 6.25 & 0.26 & 204.5 \\
\hline SPT0.3-01 & 5.59 & 0.31 & 185.3 \\
\hline SPT0.3-02 & 6.47 & 0.27 & 212.2 \\
\hline SPT0.3-03 & 5.82 & 0.31 & 192.7 \\
\hline SPT0.2-01 & 6.73 & 0.24 & 217.1 \\
\hline SPT0.2-02 & 6.52 & 0.24 & 210.6 \\
\hline
\end{tabular}

\subsection{CÁLCULO DE $K_{\text {mat }}$}

Una vez determinados los valores de $K_{r}$ y $K_{I}$, mostrados en la Tabla 5.8 y en la Tabla 5.9 respectivamente, es posible calcular los correspondientes valores de $K_{\text {mat }}$ a partir de la expresión (2.17):

$K_{r}=\frac{K_{I}}{K_{m a t}}$

Dichos valores de $K_{m a t}$ se recogen en la Tabla 5.10, los cuáles varían en función de la opción de FAD elegida y del valor de $L_{r}$ utilizado. Estos resultados serán analizados exhaustivamente en el siguiente apartado. También serán comparados con los rangos de variación de $K_{\text {mat }}$ determinados en el Capítulo 4, a partir de ensayos Charpy y de tenacidad a fractura, así como con el valor recomendado por el código ASME para el acero 15-5 PH. 
Tabla 5.10. Resultados de $K_{r}$ según la opción de FAD elegida y del valor de $L_{r}$.

\begin{tabular}{|c|c|c|c|c|c|c|}
\hline \multirow[b]{2}{*}{ Probeta } & \multicolumn{2}{|c|}{ FAD Opción 1} & \multicolumn{2}{|c|}{ FAD Opción 2} & \multicolumn{2}{|c|}{ FAD Opción 3} \\
\hline & $\begin{array}{c}\boldsymbol{K}_{\text {mat } 1 \text { trac }} \\
\left(\boldsymbol{M P a} \cdot \boldsymbol{m}^{1 / 2}\right)\end{array}$ & $\begin{array}{c}K_{m a t 1 S P T} \\
\left(M P a \cdot m^{1 / 2}\right)\end{array}$ & $\begin{array}{c}\boldsymbol{K}_{\text {mat } 2 \text { trac }} \\
\left(\mathrm{MPa} \cdot \boldsymbol{m}^{1 / 2}\right)\end{array}$ & $\begin{array}{c}\boldsymbol{K}_{m a t 2} S P T \\
\left(\boldsymbol{M P a} \cdot \boldsymbol{m}^{1 / 2}\right)\end{array}$ & $\begin{array}{c}\boldsymbol{K}_{\text {mat } 3 \text { trac }} \\
\left(\mathbf{M P a} \cdot \boldsymbol{m}^{1 / 2}\right)\end{array}$ & $\begin{array}{c}K_{m a t 3 S P T} \\
\left(M P a \cdot m^{1 / 2}\right)\end{array}$ \\
\hline SPT0.5 & 155.7 & 173.8 & 125.7 & 132.0 & 122.7 & 125.7 \\
\hline SPT0.4-01 & 350.5 & 381.8 & 263.9 & 288.9 & 222.7 & 222.7 \\
\hline SPT0.4-02 & 333.0 & 368.1 & 255.9 & 276.1 & 220.8 & 220.8 \\
\hline SPT0.4-03 & 321.7 & 354.4 & 251.9 & 271.6 & 217.8 & 217.8 \\
\hline SPT0.4-04 & 299.8 & 329.3 & 242.0 & 251.1 & 211.4 & 211.4 \\
\hline SPT0.4-05 & 305.2 & 340.8 & 246.4 & 258.8 & 215.2 & 215.2 \\
\hline SPT0.3-01 & 268.6 & 289.6 & 220.6 & 226.0 & 197.2 & 199.3 \\
\hline SPT0.3-02 & 353.6 & 400.3 & 265.2 & 312.0 & 223.3 & 225.7 \\
\hline SPT0.3-03 & 296.4 & 326.5 & 232.1 & 250.2 & 207.2 & 207.2 \\
\hline SPT0.2-01 & 355.8 & 402.0 & 268.0 & 305.7 & 226.1 & 226.1 \\
\hline SPT0.2-02 & 324.0 & 351.0 & 253.7 & 266.6 & 219.4 & 219.4 \\
\hline
\end{tabular}

\subsection{ANÁLISIS DE RESULTADOS}

En los diferentes apartados que conforman este capítulo se han obteniendo resultados parciales que han desembocado finalmente en la estimación de una serie de valores de la tenacidad a fractura del material $K_{\text {mat }}$, para cada probeta SPT ensayada. Todos estos resultados son analizados en el presente apartado.

Para la determinación de la carga aplicada sobre la probeta en el instante de inicio de la propagación de fisura $P_{a p l}$, se han propuesto tres métodos diferentes. A pesar de las diferencias existentes entre ellos, con todos se ha estimado un valor de $P_{a p l}$ para cada una de las probetas SPT ensayadas. Los ensayos se han llevado a cabo con probetas SPT prefisuradas con diferentes relaciones $a / t$, entre 0.2 y 0.55 . En el caso de las curvas correspondientes a la 
relación $a / t$ menor de 0.5 es posible establecer que el ratio entre $P_{a p l}$ y la carga máxima de la curva $P_{\max }$ está entorno al 85\%. La Figura 5.25 muestra los valores de $P_{a p l}$ de la Tabla 5.5 respecto a su correspondiente valor real de la relación $a / t$. Se puede apreciar el buen ajuste lineal obtenido, por lo que en futuros ensayos sobre probetas SPT prefisuradas será posible estimar a priori, el valor de la carga de inicio de fisuración $P_{a p l}$ para cada valor de $a / t$, mediante la expresión (5.1).

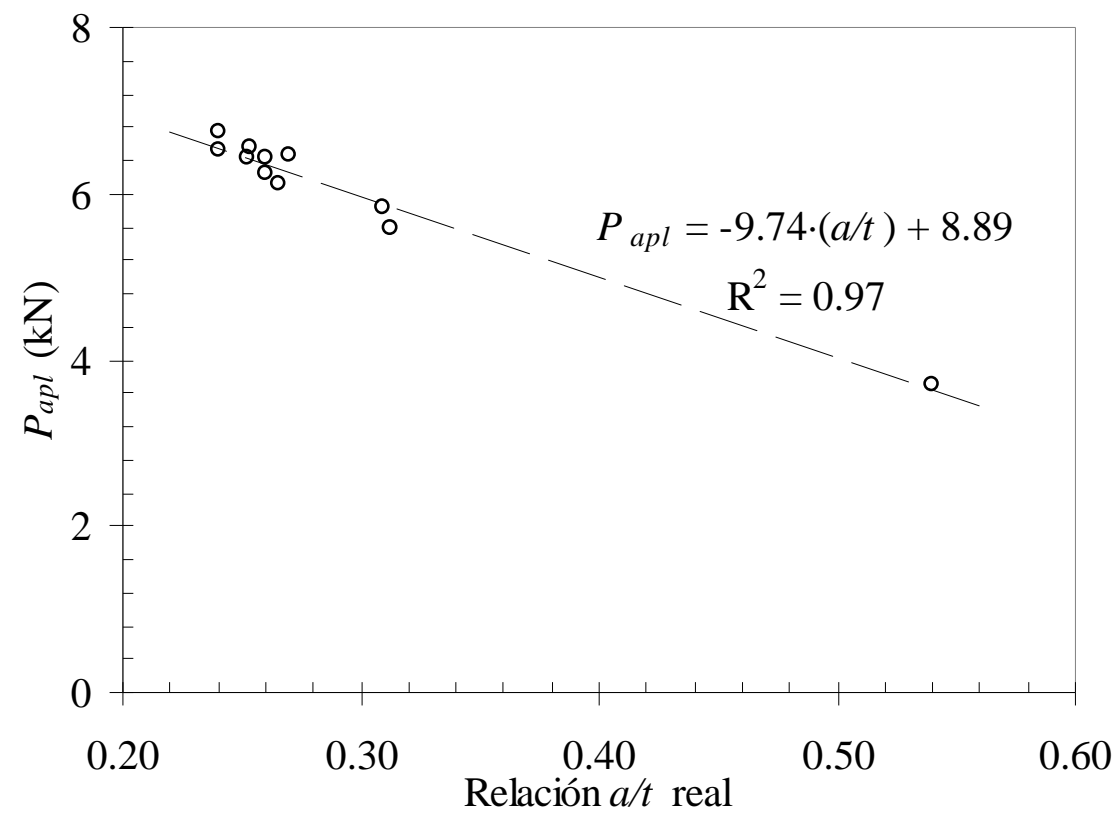

Figura 5.25. Valores de $P_{a p l}$ respecto a la relación al $t$ real.

$P_{a p l}=-9.74 \cdot(a / t)+8.89$

En la Figura 5.26 se presenta también el ajuste entre la carga de colapso plástico $P_{U}$ y la relación $a / t$ real, tomando como base para el cálculo los datos del ensayo de tracción o los estimados con el SPT convencional. De nuevo, el ajuste lineal obtenido en ambos casos es muy bueno. En este caso, en las ecuaciones correspondientes a la superficie de respuesta si se tiene en cuenta que los valores de $\sigma_{0.2}$ y $n$ permanecen constantes en cada uno de los casos, la función se convierte en linealmente dependiente de la relación $a / t$, lo que explica el buen ajuste alcanzado en la Figura 5.26. El menor valor de $P_{U}$ calculado en aquellos casos en los que se ha utilizado el SPT para estimar $\sigma_{0.2} \mathrm{y} n$, es justificable por ser menor el valor de $\sigma_{0.2}$ 
que se introduce en la función de la superficie de respuesta (3.12), 1046.7MPa (SPT-apartado 4.4) frente a los 1100MPa determinados con el ensayo de tracción (apartado 4.3).

Cabe destacar también que la pendiente de $P_{a p l}$ y de $P_{U}$ son prácticamente coincidentes. Recordemos que $P_{a p l}$ es la carga de inicio de fisuración (valor determinado experimentalmente) y que $P_{U}$ es la carga de colapso plástico de la probeta (valor determinado numéricamente).

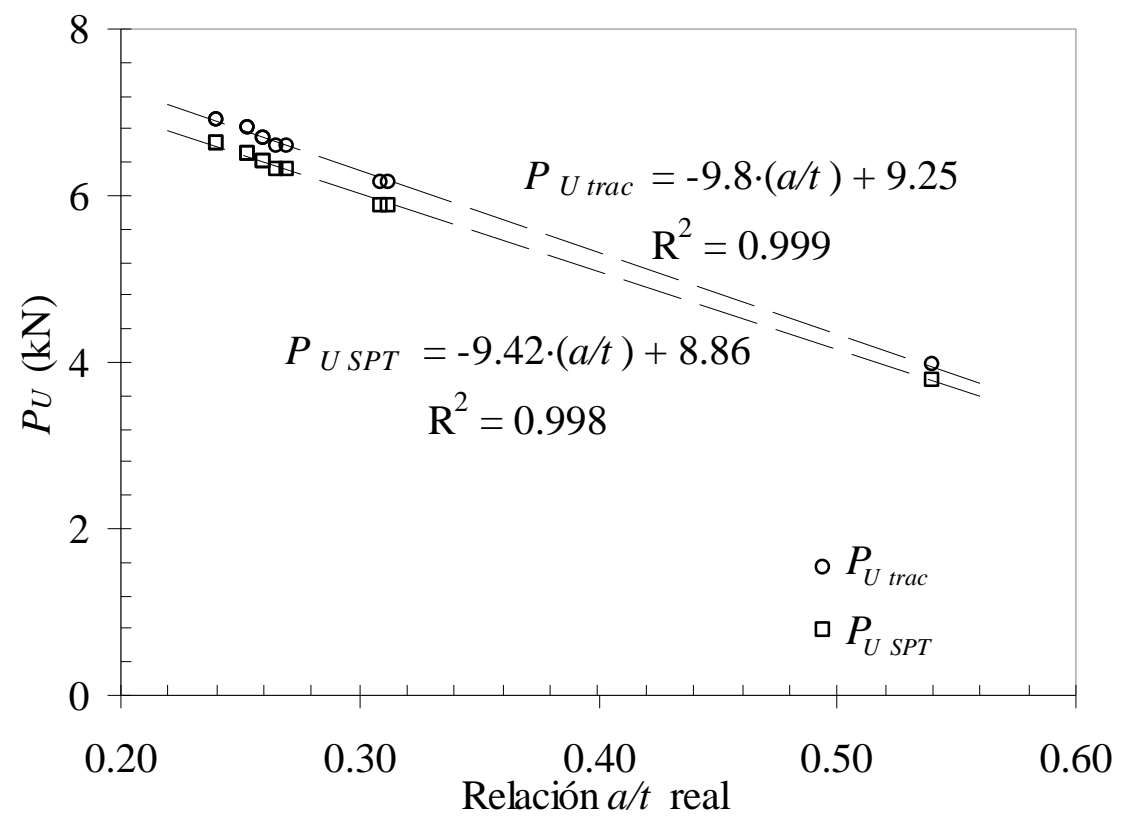

Figura 5.26. Valores de $P_{U}$ respecto a la relación a $t$ real, en función de la procedencia de los valores de $\sigma_{0.2}$ y $n$.

Una vez establecidos los valores de $P_{a p l}$ y $P_{U}$, el siguiente paso ha sido la determinación del ratio de carga $\left(L_{r}=P_{a p l} / P_{U}\right)$ para cada una de las probetas ensayadas. Sus valores han quedado recogidos en la Tabla 5.6, donde se puede observar como los valores correspondientes a la alternativa del ensayo de tracción, para determinar las propiedades elastoplásticas del material, son sensiblemente inferiores (la media es 0.95 frente a 0.99 ) a los correspondientes mediante estimación con el SPT convencional. En general, los valores de $L_{r}$ son altos y cercanos al valor de $L_{r}^{\max }$, lo cuál indica que el grado de plastificación que se alcanza en la probeta en el momento del fallo es elevado. 
Como se ha comentado anteriormente, antes de poder establecer el valor de $K_{r}$ es necesario representar el diagrama FAD según la opción que se desee aplicar. En la Figura 5.27 están representados conjuntamente los FAD determinados con las tres opciones. Es normal que conforme se pase de la Opción 1 a la 3 la zona segura del diagrama aumente, proporcionando resultados de mayor exactitud y una evaluación menos conservadora. Por otro lado, cabe destacar que la alternativa utilizada para determinar los valores de $\sigma_{0.2}$ y $n$ no influye en gran medida en la forma de las curvas de las Opciones 2 y 3 del FAD.

Con los valores de $L_{r}$ de la Tabla 5.6 queda establecida la abscisa en el diagrama FAD del instante en el que se produce el inicio de propagación de fisura, es decir, el fallo de la probeta. A partir del punto de corte de dicha abscisa con la curva del diagrama queda identificado el valor de la ordenada de dicho punto, cuyo valor corresponde al ratio de tenacidad $K_{r}$. La Figura 5.28 y la Figura 5.29 muestran los puntos de inicio de propagación para cada probeta, según la opción de FAD elegida y la alternativa empleada para obtener $\sigma_{0.2}$ y $n$, con los que se han obtenido los valores de $K_{r}$ de la Tabla 5.8. Como consecuencia de que los valores de $L_{r}$ son altos, todos los puntos representados en estas figuras están en la zona del diagrama en la que el fallo se produce con un alto grado de plastificación.

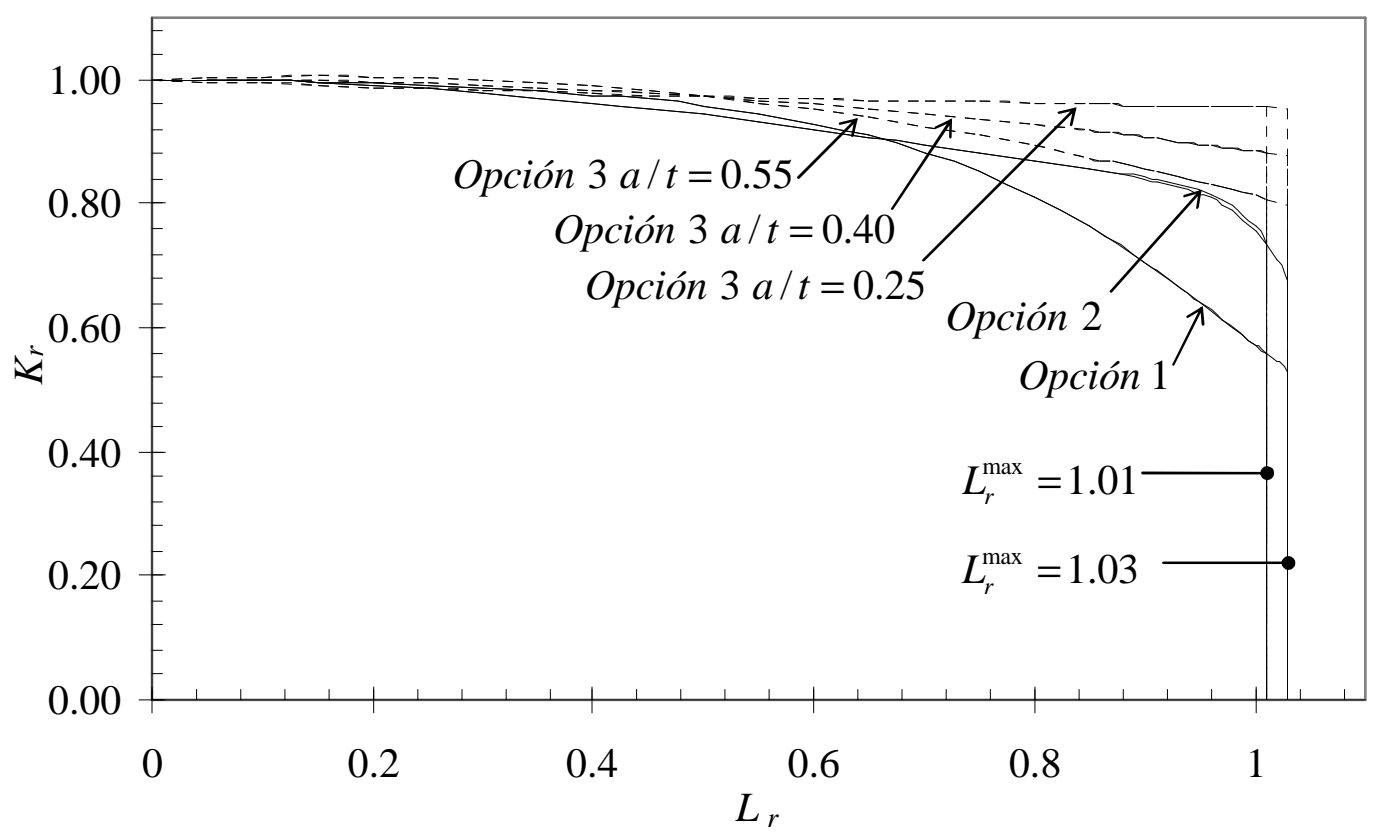

Figura 5.27. Diagramas FAD determinados en el apartado 5.5. 


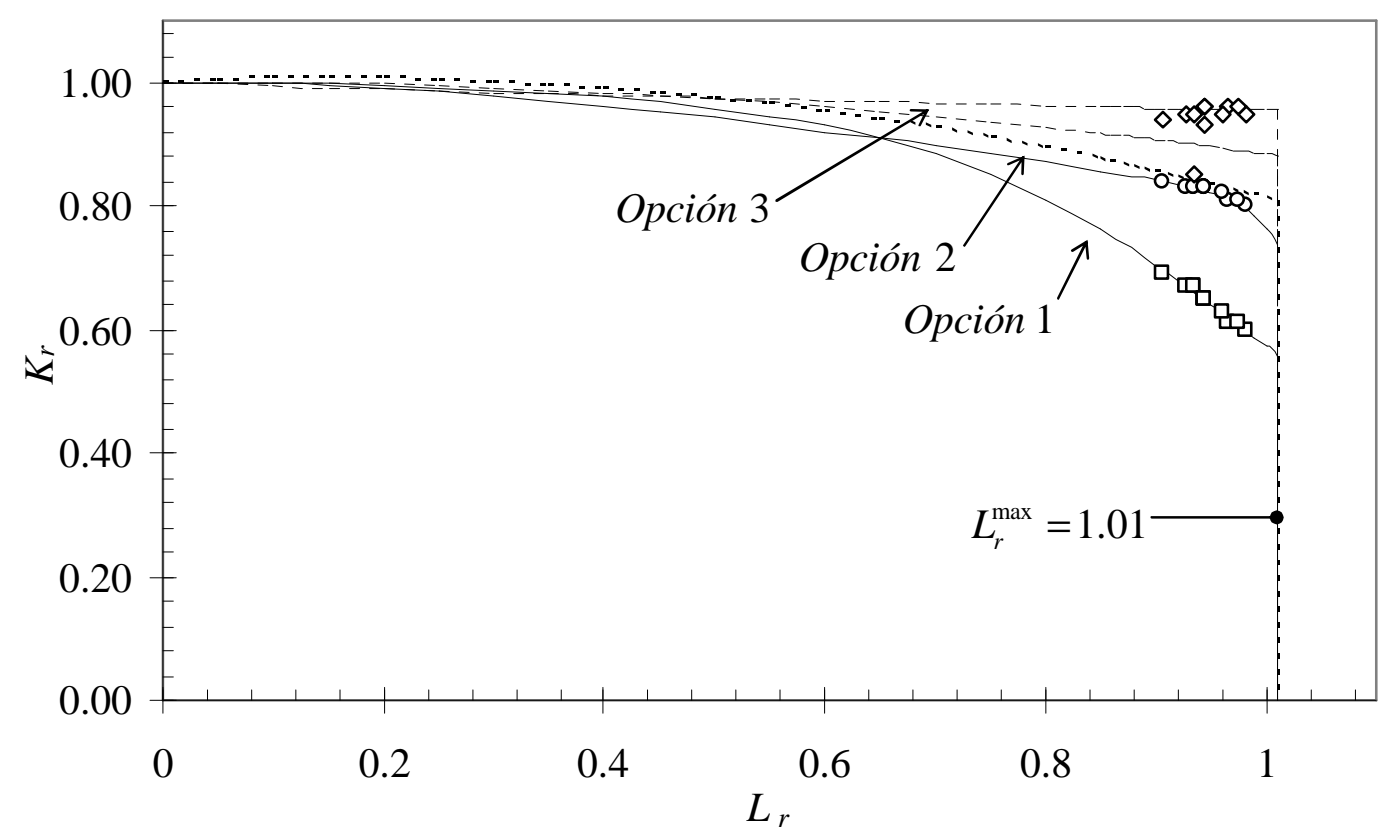

Figura 5.28. Puntos de inicio de propagación para cada probeta, según la opción de FAD elegida, a partir de los valores de $\sigma_{0.2}$ y $n$ determinados con el ensayo de tracción.

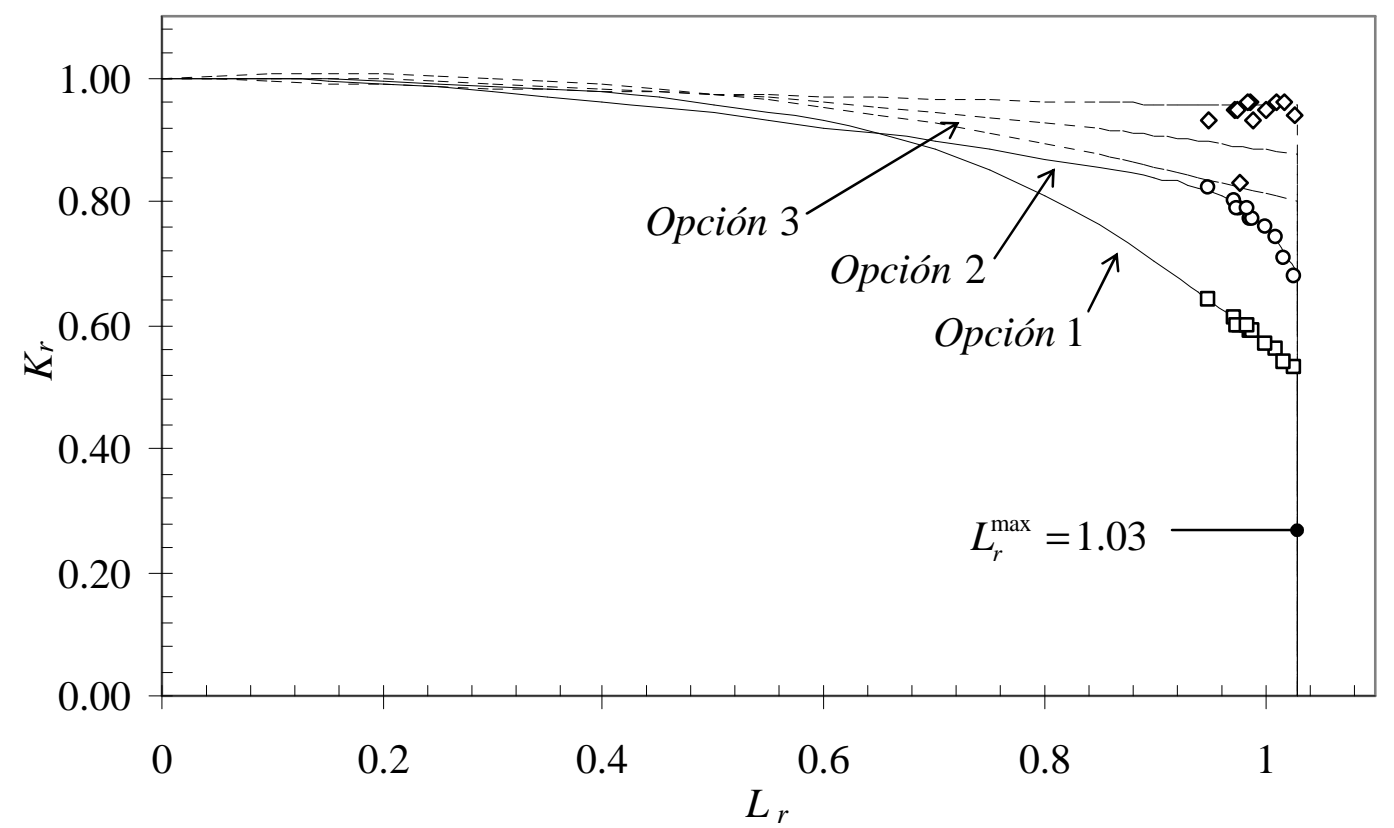

Figura 5.29. Puntos de inicio de propagación para cada probeta, según la opción de FAD elegida, a partir de los valores de $\sigma_{0.2}$ y $n$ estimados con el SPT. 
Finalmente, para concluir este capítulo, quedaría por analizar los valores estimados de la tenacidad a fractura del acero 15-5 PH, a partir de la aplicación de la metodología presentada. Como se ha podido corroborar en el apartado 5.8, para cada probeta SPT empleada, la aplicación de dicha metodología genera un total de seis valores de $K_{m a t}$ (Tabla 5.10). Estos seis valores correspondientes a cada probeta se pueden diferenciar en función de la opción de FAD elegida para su obtención, o bien, en función de la alternativa utilizada para determinar las propiedades elastoplásticas del material (ensayo de tracción o SPT).

Teniendo en cuenta esta última posibilidad se han representado los valores estimados de $K_{\text {mat }}$ en la Figura 5.30 y en la Figura 5.31, separándolos a su vez según la opción de FAD utilizada. En dichas figuras se han incluido dos franjas rayadas, las cuáles definen el rango de variación de $K_{\text {mat }}$ determinado a partir de los ensayos Charpy y de tenacidad recogidos en el Capítulo 4 de la presente Tesis, y una línea punteada correspondiente al valor recomendado por el código ASME $\left(K_{m a t}=133 \mathrm{MPa} \cdot \mathrm{m}^{1 / 2}\right)$. La franja correspondiente al ensayo de tenacidad con probetas CT es considerada como más ajustada a la hora de comparar los resultados obtenidos.

En ambas figuras se puede apreciar como la tendencia de cada conjunto de valores de $K_{\text {mat }}$ vuelve a ser lineal. Como era de esperar, se puede observar como la dispersión de resultados, así como la estimación del valor de $K_{\text {mat }}$ mejora conforme aumenta el nivel de FAD utilizado. A tenor de los resultados obtenidos se aconseja el empleo de la Opción 3 del FAD en la aplicación de la metodología desarrollada. Observando las figuras anteriores, la utilización de dicha opción con cualquier relación $a / t$, no garantiza que el valor estimado de $K_{\text {mat }}$ se encuentre dentro del rango válido de variación definido a partir del ensayo de tenacidad. Por ello, el uso de la Opción 3 de FAD junto con una relación $a / t$ entre 0.3 y 0.36 se hace necesario para estimar un valor de $K_{m a t}$ dentro del rango válido.

El efecto lineal observado en la estimación de $K_{\text {mat }}$ también está presente en los ensayos de tenacidad a fractura con probetas CT. En este caso, el efecto del espesor condiciona la validez del cálculo de $K_{I c}$. De hecho, para condiciones de tensión plana las probetas CT conducen a valores elevados de tenacidad. En este caso, resulta evidente que la elevada plastificación está condicionando el proceso. 


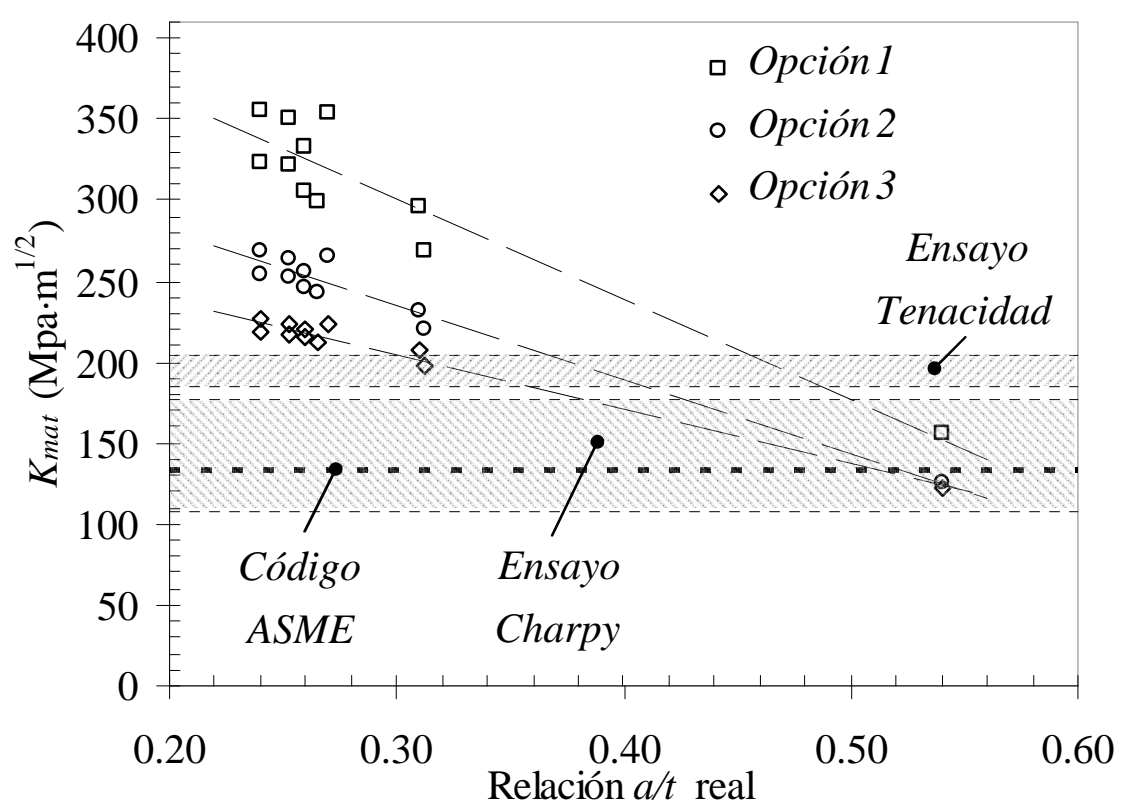

Figura 5.30. Valores de $K_{\text {mat }}$ respecto a la relación a / $t$ real, según la opción de FAD elegida, a partir de los valores de $\sigma_{0.2}$ y $n$ determinados con el ensayo de tracción.

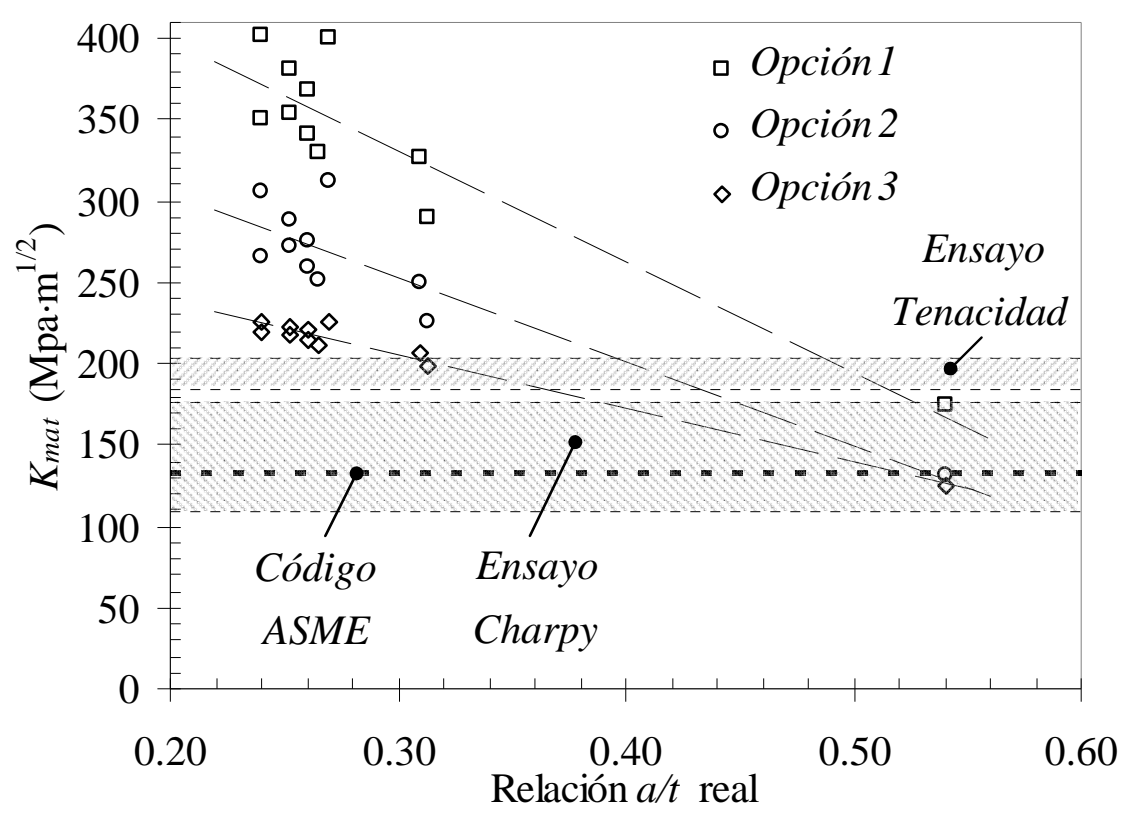

Figura 5.31. Valores de $K_{\text {mat }}$ respecto a la relación a $t$ real, según la opción de FAD elegida, a partir de los valores de $\sigma_{0.2}$ y $n$ estimados con el SPT. 
Para profundidades de fisura importantes $(a / t>0.4)$ y por lo tanto, reducido ligamento resistente, empieza a primar la elevada plastificación, poniendo en riesgo la aplicabilidad del factor de intensidad de tensiones $K_{I}$ como parámetro válido. Por el contrario, para relaciones $a / t<0.3$ donde el ligamento es mayor, las condiciones de tensión plana pueden resultar condicionantes. Un fenómeno remarcable en la geometría analizada, y que merece un análisis más exhaustivo es el hecho de que para valores de $a / t \simeq 1 / 3$ se produce un pico en el factor de intensidad de tensiones de la geometría. Ese valor coincide, con la zona de mejor estimación de la tenacidad del material.

Por último, cabe destacar que los valores de $K_{\text {mat }}$ que se encuentren por encima del rango válido de variación establecido, están sobrevalorando dicho parámetro, por lo que estaríamos del lado de la inseguridad. Todo lo contrario sucedería en el caso opuesto, donde se estaría infravalorando $K_{\text {mat }}$, encontrándonos del lado de la seguridad, como por ejemplo sucede con los resultados del ensayo Charpy, con los obtenidos con probetas SPT con una relación $a / t$ entorno a 0.5 o con el valor recomendado por el código ASME, como se puede observar en las figuras anteriores. A la vista de estos hechos, habrá que poner especial atención en aquellos casos en los que se utilicen probetas SPT prefisuradas con una relación $a / t$ menor de 0.3 , ya que son las que a priori sobrevaloran la estimación de $K_{\text {mat }}$. 



\section{CAPÍTULO 6}

\section{CONCLUSIONES Y TRABAJO FUTURO}

\subsection{CONCLUSIONES}

Como se ha comentado anteriormente, el presente trabajo viene motivado por la necesidad de dar solución al problema que presenta la estimación de las propiedades a fractura de un componente, en aquellos casos en los que no se dispone de material suficiente para llevar a cabo ensayos normalizados.

Para ello se ha elaborado una metodología, la cuál está basada en la aplicación de procedimientos de integridad estructural al ensayo SPT con probetas prefisuradas. En este trabajo se han elegido probetas SPT de dimensiones 20x20x1 $\mathrm{mm}$ con una fisura inicial del tipo longitudinal no pasante, realizada por microfisuración por láser. Las principales conclusiones que se derivan de la presente Tesis se resumen en los siguientes párrafos.

\subsubsection{De las técnicas experimentales}

- Se ha empleado la técnica de microfisuración por láser para el mecanizado de las fisuras iniciales de las probetas SPT, generando una fisura inicial del tipo longitudinal no pasante. El valor teórico de la relación $a / t$, siempre que se utilice la técnica de microfisuración por láser, hay que comprobarlo posteriormente al ensayo con un análisis SEM de la superficie de fractura de la probeta SPT. A partir de dicho análisis, 
se determina correctamente la profundidad de fisura de las probetas SPT empleadas, y en consecuencia, la relación $a / t$ real. Se ha comprobado que resulta difícil conseguir con exactitud los valores teóricos de la relación $a / t$, por lo que se puede concluir, que siempre que se aplique la técnica de fisuración por láser, será necesaria una comprobación de la profundidad real de la fisura en cada una de las probetas SPT prefisuradas.

- La realización de ensayos interrumpidos ha permitido conocer en detalle el proceso de fisuración de este tipo de probetas SPT prefisuradas. Gracias al análisis SEM y a los ensayos interrumpidos realizados es posible hacerse una idea de como es realmente la evolución de la propagación de fisura en la probeta SPT prefisurada. Dicha propagación se puede observar en la Figura 6.1, donde comenzaría en el centro del frente de grieta inicial, conforme va avanzando el ensayo se convierte en pasante, generando de esta manera dos frentes de grieta que avanzarían en sentidos opuestos hasta la zona de empotramiento de la probeta.

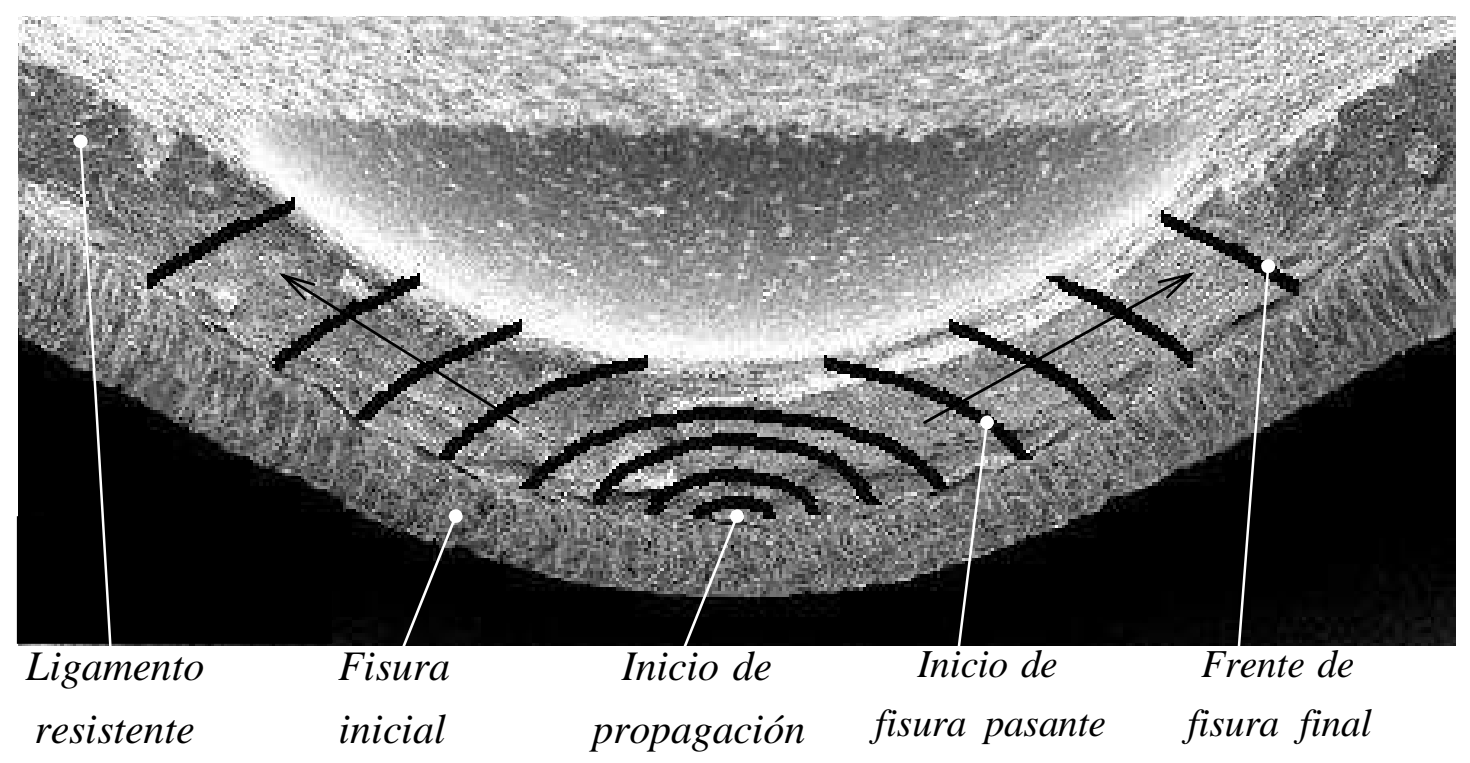

Figura 6.1. Esquema de la propagación de fisura en probetas SPT prefisuradas.

- Se han aplicado diferentes métodos para determinar la carga de inicio de fisuración en las probetas SPT prefisuradas. En particular se han realizado ensayos interrumpidos, se ha analizado la pendiente de la curva carga-desplazamiento y se ha efectuado un ensayo con descargas elásticas (método de la flexibilidad). De los tres métodos 
presentados para tal fin, el método de la flexibilidad parece a priori el más adecuado, debido principalmente a que el método de ensayos interrumpidos no permite determinar el inicio de fisuración con exactitud, además de requerir para su aplicación un análisis SEM de las probetas SPT ensayadas. Y por otro lado, el método de evaluación de la pendiente de la curva presenta el inconveniente de poder ser usado sólo con probetas con una relación $a / t<0.5$, donde la región III de la curva es lo suficientemente amplia para poder ser ajustada linealmente. El método de la flexibilidad presenta la ventaja de que la pendiente de las descargas elásticas es independiente de la forma de la curva carga-desplazamiento hasta el momento de iniciación. Es decir, no se ve influida por las diferentes regiones iniciales de la curva carga-desplazamiento. Únicamente se detecta la variación en el instante de inicio de propagación de fisura.

\subsubsection{De la simulación numérica}

- Se ha profundizado en el cálculo del valor de la carga de colapso plástico $P_{U}$ en las probetas SPT prefisuradas, en particular, para las dimensiones de 20x20x1mm y 10x10x0.5mm. Para cada una de las probetas ensayadas se ha estimado el valor de $P_{U}$ a través de la superficie de respuesta definida por la expresión (3.12), correspondiente a la probeta de dimensiones 20x20x1 $\mathrm{mm}$. Los parámetros necesarios para poder aplicar la superficie de respuesta definida por la expresión (3.12), son $a / t, \sigma_{0.2}$ y $n$. En función de la procedencia de los valores de $\sigma_{0.2} \mathrm{y} n$, es decir, si se han obtenido a partir del ensayo de tracción uniaxial o a partir del SPT, es posible estimar dos valores diferentes de $P_{U}$ para cada uno de los valores de $a / t$. La utilización de las técnicas de superficie de respuesta basadas en el diseño de experimentos supone una importante ayuda a la hora de determinar la carga de colapso plástico de la probeta SPT prefisurada. Posibilitando la aportación de la función $g\left(a / t^{*}, \sigma_{0.2}^{*}, n^{*}\right)$, con la cuál es posible establecer el valor de $P_{U}$ sin necesidad de llevar a cabo una simulación numérica, para cualquier combinación de valores de los parámetros $a / t, \sigma_{0.2}$ y $n$, siempre dentro del rango establecido para cada dimensión de probeta. En relación a 
este aspecto, se ha determinado la relación existente para el valor de $P_{U}$ entre los dos tamaños de probeta utilizados, en función del factor de forma $\Delta_{M / m}$. Este hecho permitiría extrapolar los resultados obtenidos a otras dimensiones de probeta, siempre y cuando se mantenga el factor de forma en todos los parámetros geométricos.

- Se han desarrollado expresiones que permiten determinar el factor de intensidad de tensiones para la geometría analizada. El empleo de la técnica de correlación de desplazamientos permite la evaluación de los factores de intensidad de tensiones, posibilitando en última instancia, la aportación de una expresión con la que determinar el valor de $K_{I}$, para cada tamaño de probeta $(20 \times 20 \times 1 \mathrm{~mm}$ ó 10x10x0.5mm). Dicha expresión viene dada en función de la carga aplicada y de la relación $a / t$. Durante el desarrollo de la misma, se ha observado que para cualquier carga, el máximo de la curva obtenida se encuentra entre los valores de 0.3 y 0.4 de la relación $a / t$. Estos valores definirían el valor óptimo de profundidad de fisura con el que se alcanza el valor máximo de $K_{I}$.

\subsubsection{De la metodología}

- Se ha completado un procedimiento para la estimación de las propiedades elastoplásticas del material a través del propio ensayo SPT, ya que en la gran mayoría de los casos en los que se desee aplicar la metodología presentada no se va a poseer material suficiente, con el que mecanizar probetas normalizadas de tracción para determinar dichas propiedades del material. Este procedimiento está basado en el estudio que realizaron Mao y Takahashi [4], en la mecánica de medios continuos y en la simulación numérica.

- Se ha desarrollado una metodología que combina los procedimientos de integridad estructural (diagrama FAD) y el ensayo SPT sobre probetas prefisuradas, para estimar el valor de la tenacidad a fractura del material analizado. Este trabajo se apoya en la simulación numérica de la probeta SPT prefisurada para la determinación de los valores de la carga de colapso plástico $P_{U}$ y del factor de intensidad de tensiones $K_{I}$ 
durante el proceso de carga, los cuáles son parámetros necesarios para la implementación de la metodología.

- Con el objetivo de apoyar la metodología desarrollada, ésta se ha empleado sobre probetas SPT del acero15-5 PH, caracterizado en el Capítulo 4. Los resultados obtenidos junto con su posterior análisis sientan las bases de las principales conclusiones alcanzadas en la presente Tesis. Tal y como se ha visto, tanto los resultados parciales como los correspondientes al valor de $K_{\text {mat }}$ de cada probeta SPT, se han obtenido a partir de las dos alternativas planteadas para la determinación de los parámetros elastoplásticos del material. Se puede destacar, que con los parámetros elastoplásticos determinados a partir del ensayo de tracción se mejora la dispersión y el valor de los diferentes resultados alcanzados. Este hecho se puede justificar fácilmente debido a que el procedimiento alternativo planteado al ensayo de tracción, está basado en correlaciones empíricas, por lo que hay que contar de antemano con un cierto grado de dispersión mayor. A pesar de ello, los resultados obtenidos a través de ambos procedimientos no difieren en exceso y tienen la misma tendencia.

Como se ha ratificado anteriormente, dicha tendencia de los valores de los parámetros $P_{a p l}, P_{U}$ y $K_{\text {mat }}$ respecto a la relación $a / t$ real puede ser considerada como lineal. Donde $a / t$ es inversamente proporcional a cada uno de los diferentes parámetros, es decir, si la relación $a / t$ aumenta entonces el parámetro que estemos analizando disminuye. Este hecho es necesario tenerlo en cuenta a la hora de establecer la relación $a / t$ recomendada para las probetas SPT prefisuradas.

- En los resultados obtenidos en función de la opción del FAD utilizada, se ha podido comprobar como la dispersión de resultados, así como la estimación del valor de $K_{\text {mat }}$ mejora conforme aumenta el nivel de FAD aplicado. Globalmente, la Opción 3 del FAD es la que proporciona los valores de $K_{\text {mat }}$ más cercanos al rango de variación establecido a través del ensayo de tenacidad a fractura, considerado como el rango válido de $K_{\text {mat }}$ para el acero 15-5 PH. En concreto, según la tendencia lineal comentada, los valores de $K_{\text {mat }}$ correspondientes a una relación $a / t$ entre 0.3 y 0.36 , son los que se encuentran dentro de dicho rango. 
- Por otro lado, los valores de $L_{r}$ correspondientes a cada probeta SPT ensayada son altos y cercanos al valor de $L_{r}^{\max }$, indicando que el grado de plastificación que se alcanza en la probeta en el momento del fallo es elevado. Para dichos valores no se ha observado ninguna tendencia concreta con respecto a la relación $a / t$, por lo que no se puede especificar que relación $a / t$ garantizaría un valor de $L_{r}$ bajo, que haría que el grado de plastificación no fuese tan elevado.

- También se ha observado que los resultados del ensayo Charpy y los obtenidos con probetas SPT con una relación $a / t$ entorno a 0.5 proporcionan una estimación del valor de $K_{\text {mat }}$ similar. Como se ha comentado anteriormente, dicha estimación estaría infravalorando $K_{\text {mat }}$, encontrándonos del lado de la seguridad. Todo lo contrario sucede cuando la relación $a / t$ menor de 0.3 , debido a que se sobrevaloraría $K_{\text {mat }}$, estando del lado de la inseguridad. Se recomienda por lo tanto, emplear probetas SPT prefisuradas con una relación $a / t$ real entre 0.3 y 0.36 .

\subsection{TRABAJO FUTURO}

A tenor de los resultados extraídos en la presente Tesis, la metodología desarrollada representa una herramienta útil para la estimación de la tenacidad a fractura de un material, cuando no se dispone de una cantidad suficiente para realizar ensayos convencionales, siempre y cuando se tengan en cuenta las recomendaciones realizadas anteriormente. La validación de la metodología desarrollada supone un avance importante, abriendo el camino hacia la estandarización del procedimiento, lo cuál permitiría establecer un criterio común para la evaluación de la tenacidad a fractura del material mediante el ensayo SPT sobre probetas prefisuradas. En este sentido, se van a llevar a cabo ensayos SPT prefisurados sobre probetas convencionales, es decir, de tamaño 10x10x0.5mm, que permitan evaluar el efecto que tiene sobre los resultados obtenidos el tamaño de probeta empleado. Además, se pretende aplicar la metodología propuesta a otro tipo de materiales. Siendo el comportamiento de los materiales frágiles el que presenta un mayor interés de estudio, dado que el fallo en este tipo de materiales se produce con un grado de plastificación mucho menor que en los materiales dúctiles, lo que implicaría alejarnos de la zona plástica del FAD. 
Como se ha podido comprobar, la microfisuración por láser logra una fisura afilada, pero presenta el inconveniente de no conseguir una profundidad de fisura homogénea y del valor deseado, por lo que es necesario un posterior análisis SEM de la fisura. Existen otras posibles técnicas para la generación de la fisura inicial, como el micromecanizado de alta precisión, del que se puede destacar el buen acabado superficial de la fisura y la homogeneidad conseguida de la profundidad de fisura, implicando esto la repetibilidad de las curvas obtenidas en el ensayo SPT. Como desventaja se podría decir que la forma de la fisura inicial se asemeja más al de una entalla que al de una fisura afilada, como se puede observar en la Figura 6.2. A corto plazo, se prevé el estudio de la posible implementación de las fisuras micromecanizadas como una alternativa factible a la microfisuración por láser.
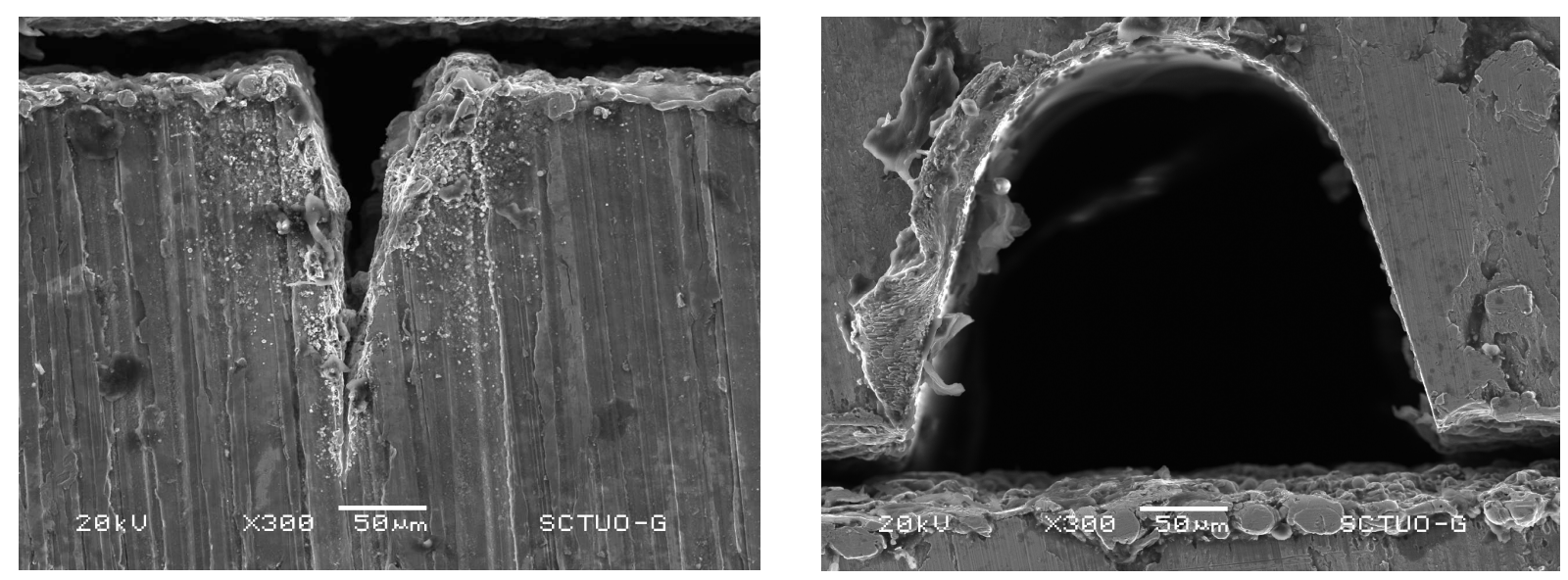

Figura 6.2. Fisuras generadas por microfisuración por láser (derecha) y por micromecanizado de alta precisión (izquierda).

Otra acción prevista a corto plazo, es la generación de una colección de curvas para la Opción 3 del FAD, en función de la relación $a / t, \sigma_{0.2}$ y $n$, con la que suplir la necesidad de llevar a cabo una simulación numérica en caso de querer aplicar dicha opción de FAD. Esta tarea es relativamente sencilla, el único inconveniente que presenta es el elevado coste computacional que conlleva.

Para concluir, cabe destacar que esta metodología abre un amplio abanico de posibilidades en cuanto a su utilización para la estimación de las propiedades a fractura a diferentes temperaturas, mediante el uso de probetas SPT prefisuradas. Otro posible campo de trabajo sería en el análisis de otro tipo de fisuras iniciales, diferentes a la longitudinal no pasante empleada para desarrollar la presente Tesis. 



\section{CAPÍTULO 7}

\section{BIBLIOGRAFÍA}

[1] J. M. Baik, J. Kameda y O. Buck, "Small Punch Test evaluation of intergranular embrittlement of an alloy steel”, Scripta Metallurgica et Materialia 17 (1983) 1443-1447.

[2] J. Kameda y O. Buck, "Evaluation of the Ductile-to-Brittle Transition Temperature Shift due to Temper Embrittlement and Neutron Irradiation by means of a Small-punch Test", Materials Science and Engineering, 83 (1986) 29-38.

[3] G.E. Lucas, A. Okada y M. Kiritani, "Parametric analysis of the disc bend test", J. Nucl. Mater. 141-143 (1986) 532-535.

[4] Mao X. y Takahashi H., "Development of a further-miniaturized specimen of 3mm diameter for TEM disk ( $\varnothing 3 \mathrm{~mm}$ ) small punch tests”, J. Nucl. Mater. 150 (1987) 42-52.

[5] Mao X., Shoji T. y Takahashi H., "Characterization of fracture behavior in small punch test by combined recrystallization —etch method and rigid plastic analysis. J Test Eval 15 (1987) 30-7.

[6] T. Misawa, T. Adachi, M. Saito y Y. Hamaguchi, "Small punch tests for evaluating ductile-brittle transition behaviour of irradiated ferritic steels", Journal of Nuclear Materials 150 (1987) 194-202. 
[7] T. Misawa, Y. Hamaguchi y M. Saito, "Stress corrosion cracking and hydrogen embrittlement studies of austenitic and ferritic steels by small punch test”, Journal of Nuclear Materials 155-157 (1988) 749-753.

[8] T. Misawa, S. Nagata, N. Aoki, J. Ishizaka y Y. Hamaguchi, "Fracture toughness evaluation of fusion reactor structural steels at low temperatures by small punch tests", Journal of Nuclear Materials 169 (1989) 225-232.

[9] T. Misawa, K. Suzuki, M. Saito y Y. Hamaguchi, "Determination of the minimum quantity of irradiated ferritic steel specimens for small punch DBTT testing”, Journal of Nuclear Materials 179-181 (1991) 421-424.

[10] T. Misawa, T. Ohtsuka, M. Seo y M. Saito, "Comparison of stress corrosion cracking susceptibility of austenitic and ferritic stainless steels in small punch testing", Journal of Nuclear Materials 179-181 (1991) 611-614.

[11] Lucas, G.E., "Review of small specimen tests techniques for irradiation testing", Metall. Trans. A 21A (1990) 1105-1119.

[12] X. Mao, M. Saito y H. Takahashi, "Small punch test to predict ductile fracture toughness $J_{I c}$ and brittle fracture toughness $K_{I c}$ ", Scripta Metallurgica et Materialia 25 (1991) 24812485.

[13] X. Mao, H. Takahashi y T. Kodaira, "Estimation of mechanical properties of irradiated nuclear pressure vessel steel by use of subsized CT specimen and small punch specimen", Scripta Metallurgica et Materialia 25 (1991) 2487-2490.

[14] X. Mao, H. Takahashi y T. Kodaira, "Supersmall punch test to estimate fracture toughness $J_{I c}$ and its application to radiation embrittlement of $2 \frac{1}{4} \mathrm{Cr}$-1Mo steel", Materials Science and Engineering AI50 (1992) 231-236.

[15] M. Suzuki, M. Eto, K, Fukaya, Y. Nishiyama, T. Kodaira, T. Oku, M. Adachi, A. Umino, I. Takahashi, T. Misawa y Y. Hamaguchi, "Evaluation of toughness degradation by small punch (SP) tests for neutron-irradiated $2 \frac{1}{4}$ Cr-1Mo steel", Journal of Nuclear Materials 179181 (1991) 441-444. 
[16] Kameda J. y Mao X., "Small-punch and TEM disk testing techniques and their application to characterization of radiation damage”, J Mater Sci 27 (1992) 983-989.

[17] J. Kameda, R. Ranjan, "Characterization of deformation and fracture behavior in amorphous and/or ceramic coatings and aluminum alloy substrates by small punch testing and acoustic emission techniques”, Materials Science and Engineering, A183 (1994) 121-130.

[18] S.-H. Chi, J.-H. Hong y In-Sup Kim, "Evaluation of irradiation effects of $16 \mathrm{MeV}$ protonirradiated $12 \mathrm{Cr}-1 \mathrm{MoV}$ steel by small punch (SP) tests", Scripta Metallurgica et Materialia, 30 $\mathrm{N}^{\mathrm{o}} 12,(1994)$ 1521-1525.

[19] E. Fleury y J.S. Ha, "Small punch tests to estimate the mechanical properties of steels for steam power plant: I. Mechanical strength", International Journal of Pressure Vessels and Piping 75 (1998) 699-706.

[20] J.S. Ha y E. Fleury, "Small punch tests to estimate the mechanical properties of steels for steam power plant: II. Fracture toughness", International Journal of Pressure Vessels and Piping 75 (1998) 707-713.

[21] Y-H. Joo, T. Hashida, H. Takahashi, K. Shimomura, "The use of small punch (bulge) tests to estimate fracture stress in the lower shelf regime" Journal of Testing and Evaluation 20 (5) (1992) 336-342.

[22] M. Afzal Khan, M. Nazeer Malik, Athar Naeem, A. ul Haq, AG. Atkins, "Computer modeling of elasto-plastic fracture mechanics of ball indentation in ductile aluminium sheet", Euro-Mat 95 Conference, Symposium D. Podova, Italia (1995) 491-494.

[23] J.H. Bulloch, "Toughness losses in low alloy steels at high temperatures: an appraisal of certain factors concerning the small punch test", International Journal of Pressure Vessels and Piping 75 (1998) 791-804.

[24] J.H. Bulloch, “The small punch toughness test: some detailed fractographic information", Int. J. Pres. Ves. \& Piping 63 (1995) 177-194.

[25] B. Ule, T. Sustar, F. Dobes, K. Milicka, V. Bicego, S. Tettamanti, K. Maile, C. Schwarzkopf, M.P. Whelan, R.H. Kozlowski y J. Klaput, "Small punch test method 
assessment for the determination of the residual creep life of service exposed components: outcomes from an interlaboratory exercise”, Nuclear Engineering and Design 192 (1999) 111.

[26] S. M. Kurtz, J. R. Foulds, C. W. Jewett, S. Srivastav y A. A. Edidin, "Validation of a small punch testing technique to characterize the mechanical behaviour of ultra-highmolecular-weight polyethylene", Biomaterials 18 (1997) 1659-1663.

[27] Foulds J.R., Woytowitz J.R., Parnell T.K. y Jewett, C.W., "Fracture toughness by small punch testing”, J. Test. Eval. 23 (1995) 3-10.

[28] Maribel L. Saucedo-Muñoz, Shi Cheng Liu, Toshiyuki Hashida, Hideaki Takahashi y Hideo Nakajima, "Correlationship between $J_{I c}$ and equivalent fracture strain determined by small-punch tests in JN1, JJ1 and JK2 austenitic stainless steels", Cryogenics 41 (2001) 713719.

[29] ASTM "E813-89 Standard test for $J_{I c}$, a measure of fracture toughness", Annual Book of ASTM Standards, 1997.

[30] ASTM "E813-81 Standard test for $J_{I c}$, a measure of fracture toughness", Annual Book of ASTM Standards, 1985.

[31] A. Shekhter, S. Kim, D.G. Carr, A.B.L. Croker y S.P. Ringer, "Assessment of temper embrittlement in an ex-service $1 \mathrm{Cr}-1 \mathrm{Mo}-0.25 \mathrm{~V}$ power generating rotor by Charpy $\mathrm{V}$-Notch testing, $K_{I c}$ fracture toughness and small punch test", International Journal of Pressure Vessels and Piping 79 (2002) 611-615.

[32] Y. Ruan, P. Spätig y M. Victoria, "Assessment of mechanical properties of the martensitic steel EUROFER97 by means of punch tests", Journal of Nuclear Materials 307311 (2002) 236-239.

[33] Jang-Bog Ju, Jae-il Jang y Dongil Kwon, "Evaluation of fracture toughness by smallpunch testing techniques using sharp notched specimens", International Journal of Pressure Vessels and Piping 80 (2003) 221-228. 
[34] ASTM "E 1921 Standard Test Method for Determination of the Reference Temperature $\mathrm{T}_{0}$ in the Transition Range", ASTM, West Conshohocken, PA, 2002.

[35] D. Finarelli, M. Roedig y F. Carsughi, "Small punch tests on austenitic and martensitic steels irradiated in a spallation environment with $530 \mathrm{MeV}$ protons", Journal of Nuclear Materials 328 (2004) 146-150.

[36] E.N. Campitelli, P. Spätig, R. Bonadé, W. Hoffelner y M. Victoria, “Assessment of the constitutive properties from small ball punch test: experiment and modeling", Journal of Nuclear Materials 335 (2004) 366-378.

[37] E.N. Campitelli, P. Spätig, J. Bertsch y C. Hellwig, “Assessment of irradiation-hardening on Eurofer97' and Zircaloy 2 with punch tests and finite-element modeling", Materials Science and Engineering A 400-401 (2005) 386-392.

[38] Eskner M. y Sandström R., "Mechanical property evaluation using the small punch test", Journal of Testing and Evaluation 32 (4) (2004) 282-289.

[39] Callaghan M.D., Yeung W.Y., Ripley M.I. y Carr D.G., "An analysis of deformation and fracture behaviour of Zircaloy-4 alloy using small punch test", Materials Science Forum 475479 (II) (2005) 1415-1420.

[40] Komazaki S.-I., Shoji T. y Takamura K., "Evaluation of thermal aging embrittlement in directionally solidified Ni-base superalloy by small punch test", Journal of Engineering Materials and Technology, Transactions of the ASME 127 (4) (2005) 476-482.

[41] Dunne N.J., Leonard D., Daly C., Buchanan F.J. y Orr J.F., "Validation of the smallpunch test as a technique for characterizing the mechanical properties of acrylic bone cement", Proceedings of the Institution of Mechanical Engineers, Part H: Journal of Engineering in Medicine 220 (1) (2006) 11-21.

[42] Nakata T., Komazaki S.-I., Nakajima M., Kohno Y., Tanigawa H., Shiba K. y Kohyama A., "Evaluation of high-temperature tensile properties of reduced activation ferritic steels by small punch test", Journal of the Japan Institute of Metals 70 (8) (2006) 642-645. 
[43] Budzakoska E., Carr D.G., Stathers P.A., Li H., Harrison R.P., Hellier A.K. y Yeung W.Y., "Predicting the J integral fracture toughness of Al 6061 using the small punch test", Fatigue and Fracture of Engineering Materials and Structures 30 (9) (2007) 796-807.

[44] Hyde T.H., Sun W. y Williams J.A., "Requirements for and use of miniature test specimens to provide mechanical and creep properties of materials: A review", International Materials Reviews 52 (4) (2007) 213-255.

[45] Kim M.-C., Lee J.B., Oh Y.J. y Lee B.S., "Evaluation of critical fracture stress in low alloy steels by finite element analysis of small punch test”, Key Engineering Materials 353358 (PART 1) (2007) 416-419.

[46] Sunjaya D., Wei T., Harrison R. y Yeung W.Y., "Finite element modelling of small punch test on 304H stainless steel”, Key Engineering Materials 345-346 II (2007) 1165-1168.

[47] Wang Z., Shi H., Lu J., Shi P., Ma X., "Small punch testing for assessing the fracture properties of the reactor vessel steel with different thicknesses”, Nuclear Engineering and Design 238 (2008) 3186-3193.

[48] Jin Sik Cheon y In Sup Kim, "Evaluation of thermal aging embrittlement in CF8 duplex stainless steel by small punch test”, Journal of Nuclear Materials 278 (2000) 96-103.

[49] S.-H. Song, R.G. Faulkner, P.E.J. Flewitt, R.F. Smith y P. Marmy, "Temper embrittlement of a CrMo low-alloy steel evaluated by means of small punch testing", Materials Science and Engineering A281 (2000) 75-81.

[50] S.-H. Song, R.G. Faulkner, P.E.J. Flewitt, P. Marmy y L.-Q. Weng, "Small punch test evaluation of neutron-irradiation-induced embrittlement of a $\mathrm{Cr}-\mathrm{Mo}$ low-alloy steel", Materials Characterization 53 (2004) 35-41.

[51] X. Jia y Y. Dai, "Small punch tests on martensitic/ferritic steels F82H, T91 and OptimaxA irradiated in SINQ Target-3”, Journal of Nuclear Materials 323 (2003) 360-367.

[52] Min-Chul Kim, Yong Jun Oh y Bong Sang Lee, "Evaluation of ductile-brittle transition temperature before and after neutron irradiation for RPV steels using small punch tests", Nuclear Engineering and Design 235 (2005) 1799-1805. 
[53] I. Serre y J.-B. Vogt, "Liquid metal embrittlement of T91 martensitic steel evidenced by small punch test”, Nuclear Engineering and Design 237 (2007) 677-685.

[54] F. Dobes y K. Milicka, "Small punch testing in creep conditions", J Test Eval 29 (2001) $31-35$.

[55] F. Dobes y K. Milicka, "On the Monkman-Grant relation for small punch test data", Materials Science and Engineering A336 (2002) 245-248.

[56] F. Dobes, K. Milicka y P. Kratochvíl, "Small punch creep in Fe28Al3Cr0.02Ce alloy”, Intermetallics 12 (2004) 1397-1401.

[57] Zhen Yang y Zhi-wen Wang, "Relationship between strain and central deflection in small punch creep specimens", International Journal of Pressure Vessels and Piping 80 (2003) 397-404.

[58] Zhai PC, Hashida T, Komazaki S y Zhang QJ., "Numerical analysis for small punch creep tests by finite-element method", J. Test Eval. 32 (2004) 298-303.

[59] Zhai P.-C., Chen G., Hashida T. y Zhang Q.-J., "Evaluation on small punch creep test by finite element method”, Key Engineering Materials 297-300 I (2005) 377-383.

[60] Sugimoto T., Komazaki S.-I. y Misawa T., "Evaluation of DBTT and creep properties of aged main valve casing by using small punch specimens”, Key Engineering Materials 297300 III (2005) 1470-1476.

[61] Chen G., Zhai P.-C. y Shao A.-J., “Appraisement of creep properties of $12 \mathrm{Cr} 1 \mathrm{MoV}$ steel by small punch creep test method", Materials Science Forum 492-493 (2005) 545-550.

[62] Baek S.-S., Kwon I.-H., Lee D.-W., Yang S.-M. y Yu H.-S., "Characterization of creep resistance for local structure of power plant weldment using SP-Creep technique", Key Engineering Materials 326-328 I (2006) 643-648.

[63] Nakata T., Komazaki S.-I., Kohno Y., Shiba K., Kohyama A. y Hashida T., "Evaluation of creep properties of reduced activation ferritic steels by small punch creep test", Journal of the Japan Institute of Metals 70 (2) (2006) 130-133. 
[64] Komazaki S.-I., Sugimoto T., Hasegawa Y. y Kohno Y., "Damage evaluation of a welded joint in a long-term service-exposed boiler by using a small punch creep test", ISIJ International 47 (8) (2007) 1228-1233.

[65] Gonzalez-Posada M.A., "Influencia de la sustentación hiperestática en el criterio Fuga antes que Rotura aplicado a sistemas de tuberías en régimen elastoplástico", Tesis Doctoral, Escuela Técnica Superior de Ingenieros Industriales, Universidad de Cantabria (2004).

[66] Cicero S., "Evaluación de la integridad estructural de componentes sometidos a condiciones de bajo confinamiento", Tesis Doctoral, Universidad de Cantabria (2007).

[67] Wilkoswski G., Ahmad J., Barnes D., Brust F., Ghadiali N. y Guerreri D., "Degraded piping program - Phase II progress”, Nucl. Engng. Des. 98 (1987) 195-217.

[68] Kiefner J.F., Maxey W.A., Eiber R.J. y Duffy A.R., "Failure stress loads of flaws in pressurized cylinders”, Philadelphia: ASTM STP; Vol. 536 (1973) 461-81.

[69] Hahn G.T., Sarrate M. y Rosenfeld A.R., "Criteria for crack extension in cylindrical pressure vessels”, Int. J. Fract. Mech. 5 (1969) 187-210.

[70] Golembiewski H.J. y Vasoukis G., "On the required toughness for the application of the net section criterion on the nuclear power plant components", Nucl. Engng. Des. 87 (1985) 67-71.

[71] Golembiewski H.J. y Vasoukis G., "Influence of material properties and geometry on the limit load behaviour of flawed structures", Int. J. Pressure Vessel Piping 31 (1988) 131-140.

[72] Milne I., Ainsworth R.A., Dowling A.R. y Stewart A.T., "Assessment of the integrity of structures containing defects”, International Journal of Pressure Vessels and Piping 32 (1988) 3-104.

[73] "Assessment of the integrity of structures containing defects", CEGB Report R/H/R6, (1976); Revision 1, (1977); Revision 2, (1980); Revision 3, (1986).

[74] R6 Revision 3, "Assessment of the integrity of structures containing defects", British Energy Generation Ltd, Amendment 10, May 1999. 
[75] R6, "Assessment of the integrity of structures containing defects", British Energy Generation, Report R/H/R6, Revision 4 (2001).

[76] British Standard BS 7910, "Guide on methods for assessing the acceptability of flaws in metallic structures", BSI, London (2000).

[77] PD 6493, "Guidance methods for assessing the acceptability of flaws in fusion welded structures", BSI (1975).

[78] Andersson P., Bergman M., Brickstad B., Dahlberg L., Sattari-Far I. y Nilsson F., “A procedure for safety assessment of components with cracks - handbook", SAQ Kontroll AB, Stockholm, Sweden (1996) SAQ/FoU-Report 96/08.

[79] Bergman M., "User's manual SACC version 4.0", SAQ Kontroll AB, Stockholm, Sweden (1996) SAQ/FoU-Report 96/09.

[80] "Fitness for service guide", EXXON Chemical (1995).

[81] "Fitness for service evaluation procedures for operating pressure vessels, tanks and piping in refinery and chemical service", Draft $n^{\circ} 5$, Consultants report, The Materials Properties Council, October 1995.

[82] ASME, API 579-1/ASME FFS-1, in Fitness-For-Service, American Society of Mechanical Engineers (2007).

[83] Shih C.F. y Hutchinson J.W., "Fully plastic solutions and large-scale yielding estimates for plane stress crack problems", Journal of Engineering Materials and Technology 98 (1976) 289-295.

[84] Kumar V., German M.D. y Shih C.F., "An engineering approach for elastic-plastic fracture analysis", General Electric Company, NP-1931, Research Project 1237-1 Topical Report (1981).

[85] Schwalbe K.-H., Neale B.K. y Heerens J., "EFAM-GTP 94: the GKSS test procedure for determining the fracture behaviour of materials", GKSS-Research Centre, Report 94/E/60 (1994). 
[86] Schwalbe K.-H., Kim Y.J., Hao S., Cornec A. y Koçak M., "EFAM ETM-MM 96: the ETM method for assessing the significance of crack-like defects in joints with mechanical heterogeneity (strength mismatch)", GKSS-Research Centre, Report 97/E/9 (1997).

[87] Schwalbe K.-H., Zerbst U., Kim Y.-J., Brocks W., Cornec A., Heerens J. y Amstutz H., "EFAM ETM 97: the ETM method for assessing crack-like defects in engineering structures", GKSS-Research Centre, Report 98/E/6 (1998).

[88] Schwalbe K.-H., "EFAM 96. The Engineering Flaw Assessment Method (EFAM)", Fatique Fract. Eng. Mater. Struct. 21 (1998) 1203-1213.

[89] SINTAP, "Structural integrity assessment procedure for European industry", SINTAP BRITE-EURAM Project BRPR-CT95-0024 (1999).

[90] www.eurofitnet.org/sintap_index.html

[91] FITNET, "European Fitness-for-Service Network”, EU’s Framework 5, Proposal No. GTC1-2001-43049, Contract No. G1RT-CT-2001-05071.

[92] www.eurofitnet.org

[93] ASME Boiler and Pressure Vessel Code, Section XI, "Rules for In-Service Inspection of Nuclear Power Plant Components", The American Society of Mechanical Engineers (1995).

[94] Turner C.E., “A J-based fracture safe estimation procedure, En j, with applications including estimation of the maximum load for ductile tearing", en: Larsson LH. (Ed.), Elastic-plastic fracture mechanics (1985) 411-426.

[95] Bloom J.M. y Malik S.N., "Procedure for the Assessment of the Integrity of Nuclear Pressure Vessels and Piping Containing Defects", EPRI Topical Report NP-2431, Research Project 1237-2, Electric Power Research Institute, CA, USA (1982).

[96] Bloom J.M., “Deformation plasticity failure assessment diagram”, en: Newman Jr. J.C., y Loss F.J., Editors, Elastic-plastic fracture mechanics technology, ASTM STP 896, American Society for Testing and Materials, Philadelphia (1985) 114-127. 
[97] Ramberg W. y Osgood W. R. "Description of stress-strain curves by three parameters, Technical Note No. 902", National Advisory Committee for Aeronautics (1943), Washington DC.

[98] MSC.Marc Volume A, “Theory and User Information”, Chapter 5 Fracture Mechanics.

[99] Khuri A. I. y Cornell J. A., "Response surfaces. Design and analyses", Statistics: Textbooks and monographs. 81, Marcel Dekker (1987). New York.

[100] Kuehl R. O., "Diseño de experimentos”, International Thomson (2001).

[101] Montgomery D. C, “Diseño y análisis de experimentos”, Grupo Editorial Iberoamérica (1991).

[102] Mathieu D. et al., NEMRODW, LPRAI, Marsella. http://www.nemrodw.com.

[103] Shih C.F., de Lorenzi H.G. y German M.D., "Crack Extensión Modeling with Singular Quadratic Isoparametric Elements", International Journal of Fracture 12 (1976) 647-651.

[104] Barsoum R.S., "On the use of isoparametric finite elements in linear fracture mechanics”, Int. J. for Numerical Methods in Engineering 10 (1976) 25-37.

[105] Raju I.S., "Calculation of Strain-Energy Release Rates with Higher Order and Singular Finite Elements”, Engineering Fracture Mechanics 28 (1987) 251-274.

[106] Rice J. R., "A Path Independent Integral and the Approximate Analysis of Strain Concentration by Notches and Cracks”, Journal of Applied Mechanics 35 (1968) 379-386.

[107] Bittencourt T.N., Wawrzynek P.A., Ingraffea A.R. y Sousa J.L.A., "Quasi-Automatic Simulation of Crack Propagation for 2D LEFM Problems”, Eng. Fract. Mech. 55 (1996) 321334.

[108] Ansys Inc. V11.0, User Manual, (2008).

[109] Tada H., Paris P.C. y Irwin G.R., "The Stress Analysis of Cracks Handbook. Third Edition”, ASME International (2000). 
[110] ASTM, "E 8M Standard Test Methods of Tension Testing of Metallic Materials [Metric]" Annual Book or ASTM Standards, American Society for Testing and Materials, Vol. 3.01.

[111] ASTM "E 23-05 Standard Test Methods for Notched Bar Impact Testing of Metallic Materials" Annual Book or ASTM Standards, American Society for Testing and Materials.

[112] ASTM "E 399-90 (1997) Test Method for Plane-Strain Fracture Toughness of Metallic Materials" Annual Book or ASTM Standards, American Society for Testing and Materials.

[113] ASTM "E 1820-05 Standard Test Method for Measurement of Fracture Toughness" Annual Book or ASTM Standards, American Society for Testing and Materials. 